Facultad de Ciencias Astronómicas y Geofísicas

UNIVERSIDAD NACIONAL DE LA PLATA

\title{
DETERMINACIÓN DE LA ESTRUCTURA CORTICAL EN EL SECTOR ARGENTINO DE LA ISLA GRANDE DE TIERRA DEL FUEGO
}

\author{
CAROLINA BUFFONI
}

Directores

Dra. Nora C. Sabbione

Dr. Martin Schimmel

Tesis presentada para optar por el grado de DOCTORA EN GEOFÍSICA 


\title{
Determinación de la estructura cortical en el sector argentino de la Isla Grande de Tierra del Fuego
}

\author{
Carolina Buffoni
}

\section{Directores:}

- Dra. Nora Cristina Sabbione

Facultad de Ciencias Astronómicas y Geofísicas. Universidad Nacional de la Plata.

- Dr. Martin Schimmel

Instituto de Ciencias de la Tierra Jaume Almera (ICTJA). CSIC, España.

\section{Tribunal Examinador:}

- Dra. Patricia Gauzellino (presidente del Tribunal)

Facultad de Ciencias Astronómicas y Geofísicas. Universidad Nacional de la Plata.

- Dra. Patricia Alvarado

CIGEOBIO. Universidad de San Juan.

- Dra. Diana Comte

Facultad de Ciencias Físicas y Matemáticas. Universidad de Chile. 
A Sebastián, Matías y Goyo 
En primer lugar, quiero agradecer a mis directores Nora y Martin, por haberme guiado y acompañado en este trabajo. En particular a Nora, gracias por la paciencia, los consejos e inquietudes planteadas y por confiar en mi para poder desarrollar este trabajo. A Martin, por haber aceptado ser parte de este trabajo a mitad de camino, por enseñarme siempre a ver la mitad del vaso lleno, por contagiarme su entusiasmo y por brindarse en muchas ocasiones como un amigo.

Al personal de la Estación Astronómica de Río Grande (EARG), por proveer los datos necesarios para el desarrollo de esta Tesis. En particular a Gerardo Connon y a José Luis Hormaechea por estar presentes siempre respondiendo a mis consultas.

Al INPRES, en especial a Marcelo Moreno, por proveer los datos de la estación USHA utilizada en este trabajo.

A la Facultad de Ciencias Astronómicas y Geofísicas de la Universidad Nacional de La Plata, por brindarme un lugar de trabajo durante los años que estuve en Argentina.

Al CONICET, por la financiación parcial de esta Tesis.

A mis compañeros del Departamento de Sismología. En especial a Gabriela y Laura por los consejos, charlas y correos electrónicos cuando estaba fuera del país. A Gabi, gracias por su gran aporte para que pudiese realizar una estancia de investigación en Granada. A Lau, por compartir todos sus conocimientos acerca de las Funciones del Receptor y la inversión de las curvas de dispersión. Gracias a ambas por compartir siempre su experiencia.

Al Instituto Andaluz de Geofísica, por proveer un lugar para mi estancia de investigación. En particular a Jesús Ibañez, por aceptar ser director de dicha estancia.

Al Instituto de Ciencias de la Tierra Jaume Almera (CSIC), por brindarme un lugar de trabajo y todo el equipamiento necesario para que pueda seguir desarrollando esta Tesis sin financiación desde Barcelona. Martin, gracias de nuevo por esto!.

A Luciana Bonatto, por recibirme los primeros días en Granada, presentarme el Instituto y luego a uno de mis directores.

A Robert Herrmann, por su invalorable ayuda con la implementación de su programa, los consejos y el gran interés demostrado en la zona de estudio de esta Tesis.

A Alejandro Tassone, por su colaboración en la interpretación de los resultados de esta Tesis.

A mi amiga Silvana Spagnotto, quien siempre tiene un consejo optimista que compartir.

A mis amigas de Argentina y España, por alentarme y repetirme siempre la frase "dale que es un 
último esfuerzo, vos podés!”.

A mi suegra y a mi cuñada, por estar siempre que las necesité para ocuparse de Matías.

A mi querido Goyo, quien me acompañó durante mi carrera de Geofísica y luego durante el Doctorado. Siempre al lado mío cuando trabajaba desde casa, gracias por exigir esos cortos paseos que me ayudaban a despejar un poco la mente.

A mi familia, mis padres, Graciela y Héctor, todo lo que soy se los debo a ellos, gracias por estar siempre presentes aún en la distancia, por la contención y el apoyo en momentos difíciles. Por ejercer el título de abuelos intensivos estas últimas semanas para que yo puediera terminar la Tesis. A mis hermanos, por estar siempre con algún mensajito separados miles de kilómetros y por demostrarme que se puede terminar una Tesis Doctoral.

Y por último a los pilares de mi vida. A mi hijo Matías, gracias por ayudarme a superar mis miedos y enseñarme a compaginar la maternidad con este trabajo, por regalarme esas sonrisas en momentos de preocupación, por esos ratos de juego que me ayudaban a relajar la mente y por recordarme todos los días lo que realmente importa en esta vida. A mi marido Sebastián, por su apoyo y amor constante e incondicional, por ser el sustento económico de la familia permitiéndome terminar esta Tesis, por ser mi ejemplo cotidiano de perseverancia, por alentarme y apoyarme en cada decisión que tomaba y por su gran paciencia. A ellos mis gracias infinitas por haberme dado la fuerza y energía necesaria para poder terminar esta Tesis.

Carolina Buffoni

15 de diciembre de 2016 
Este trabajo de Tesis tiene como objetivo principal realizar una estimación de la estructura de la corteza del sector oriental de la Isla Grande de Tierra del Fuego (TdF). En zonas sísmicamente activas, como es el caso de TdF, es importante contar con modelos de velocidades sísmicas que se ajusten lo más posible a la estructura real de la zona, en principio, para que la localización de los sismos locales se pueda realizar con el mínimo error. A efectos de realizar el trabajo se ha contado con datos de cuatro estaciones sismológicas de banda ancha instaladas en la Isla. La zona de estudio presenta un escenario de gran interés ya que su ambiente geotectónico está caracterizado por la conjunción de tres placas tectónicas: la Antártica, la Sudamericana y la de Scotia. En particular las últimas dos definen un sistema de margen transformante que produce fallas con un movimiento sinestral E-O como es el sistema de fallas Magallanes-Fagnano que divide a la Isla en dos bloques continentales. A pesar de que la sismicidad actual de TdF está caracterizada por sismos de baja a mediana magnitud, registrados a partir de la instalación de las estaciones utilizadas, la sismicidad histórica incluye dos eventos de magnitud 7.5 y 7.8 ocurridos el 17 de diciembre de 1949.

Para llevar a cabo el objetivo propuesto se han utilizado dos técnicas que requieren de la información contenida en los registros sismológicos de una manera diferente pero que se pueden complementar. En una primera etapa, se aplica el método de las Funciones del Receptor (FRs), siendo una de las metodologías más utilizadas cuando se quiere estimar la discontinuidad de Mohorovicic y la relación de velocidades sísmicas $\mathrm{V}_{\mathrm{P}} / \mathrm{V}_{\mathrm{S}}$ de la corteza en el sitio en que está ubicada la estación sismológica. A partir de las FRs calculadas se realizan estimaciones del espesor cortical y de la relación de las velocidades sísmicas en cada estación considerando dos métodos: el "H-k stacking” y la inversión mediante el “Neighbourhood Algorithm” (NA). El primero consiste en apilar las amplitudes de las FRs en los tiempos de arribo calculados de las fases Ps, PpPs, PpSs+PsPs para distintos valores de espesor cortical $\mathrm{H}$ y de $\mathrm{V}_{\mathrm{P}} / \mathrm{V}_{\mathrm{s}}$. Mediante el segundo método NA, se lleva a cabo la inversión no-lineal de las FRs realizando un ajuste en cada iteración de un modelo inicial a través de sucesivas capas con aumentos o descensos graduales de la velocidad. La variabilidad de los modelos obtenidos en esta etapa indican que el ambiente geotécnico de esta zona es complejo. Los valores de espesor cortical y de la relación de velocidades sísmicas $\mathrm{V}_{\mathrm{P}} / \mathrm{V}_{\mathrm{S}}$ obtenidos a partir del primer método varían en el rango 24.8-34.8 km y 1.56-1.80, respectivamente, dependiendo de la estación y del grupo de eventos utilizados. En cuanto a la segunda técnica, el 
espesor cortical estimado varía desde 24.5 a $39.2 \mathrm{~km}$ y la relación $\mathrm{V}_{\mathrm{P}} / \mathrm{V}_{\mathrm{S}}$ se estimó en el rango 1.691.88. Estos valores obtenidos son acordes con la presencia de diferentes ambientes geológicos, sistemas de fallas y estructuras plegadas presentes en la Isla. Los valores de espesor cortical obtenidos en la mayoría de las estaciones sismológicas, mediante las dos técnicas, indican que la profundidad de la Moho en el sector oriental de TdF aumenta de norte a sur. Además, los valores de espesor cortical determinados con ambas técnicas, son similares a valores obtenidos en estudios previos realizados mediante otras metodologías en zonas cercanas al área de estudio de este trabajo.

En una segunda etapa se aplica el método de interferometría sísmica (SI). Mediante esta técnica es posible estimar la estructura a través del ruido sísmico o ruido ambiente. A diferencia de la técnica de las FRs, el método de SI no requiere la ocurrencia de terremotos. El concepto se basa en la extracción de la Función de Green Empírica (FGE) entre dos receptores a partir de la correlación cruzada de registros continuos de ruido. Mediante este método es posible obtener información de las ondas superficiales y calcular curvas de dispersión que caracterizan la estructura media entre cada par de estaciones. A partir de los registros continuos de ruido obtenidos en cuatro estaciones sismológicas utilizadas se obtienen seis trayectorias de ondas superficiales. En particular, se considera el cálculo de velocidades de grupo de la onda Rayleigh. Posteriormente las curvas de dispersión se invierten mediante una inversión iterativa ponderada. Teniendo en cuenta que la distancia entre estaciones en la región de estudio varía entre 50 y $100 \mathrm{~km}$, las velocidades que se obtienen aportan información de la estructura de las capas más superficiales de la corteza en TdF, en particular se obtuvieron valores de velocidad de onda $S$ hasta una profundidad de $10 \mathrm{~km}$.

La estructura superficial y local obtenida en la estación DSPA a partir de la inversión de FRs está caracterizada por velocidades de onda $\mathrm{S}$ en el rango 1.75-1.80 km/s en los primeros $2 \mathrm{~km}$ de corteza. Por otro lado, la estructura media obtenida a partir de la inversión de las curvas de dispersión calculada en los trayectos DSPA-TRVA, DSPA-ELCA y DSPA-USHA, presenta valores de $V_{\mathrm{S}}$ entre 2.3 y $2.6 \mathrm{~km} / \mathrm{s}$ para las mismas profundidades. En el caso de la estación ELCA, ubicada en el centro de la zona de estudio, la estructura local de onda S está caracterizada por valores de 2.4 $\mathrm{km} / \mathrm{s}$ en las capas más superficiales. Estos resultados obtenidos, en la zona norte y centro del área de estudio, se correlacionan con la presencia de sedimentos del Terciario y Cretácico superior. En el caso de las estaciones ubicadas en la zona sur (TRVA y USHA), se observan valores de $V_{\mathrm{s}}>3 \mathrm{~km} / \mathrm{s}$ en las capas más superficiales de los modelos obtenidos en ambas estaciones mediante la inversión de FRs. La estructura media obtenida entre TRVA y USHA a partir de la inversión de la curva de dispersión para dicho trayecto, muestra valores de velocidad de onda $\mathrm{S}$ en el rango 3.3-3.7 km/s, en los 2 primeros km de corteza. Dicho aumento de velocidad observado en la zona sur de la Isla es acorde con la presencia de rocas del basamento Paleozoico presente entre ambas estaciones. A profundidades un poco mayores (entre 5 y $8 \mathrm{~km}$ ), se observa un aumento de velocidad en varios de los modelos obtenidos con ambas técnicas que parece estar relacionado con el basamento de la zona de estudio, sugiriendo un aumento de su profundidad de norte a sur. Además, en algunos de 
los modelos obtenidos mediante la técnica NA, se distingue un elevado contraste positivo de velocidad entre los 15 y $17 \mathrm{~km}$ de profundidad que podría estar asociado con la presencia de un sub-basamento en la zona de estudio.

De esta manera los resultados obtenidos con todas las metodologías implementadas se combinan a efectos de caracterizar la corteza del sector oriental de la Isla Grande de Tierra del Fuego. Los resultados presentados en esta Tesis aportan nueva información sobre la estructura cortical de la zona y se espera que contribuyan al desarrollo de futuros trabajos con el fin de profundizar el conocimiento de la estructura y mitigar el impacto de futuros terremotos. 


\section{CAPÍTULO I: Introducción general}

1.1 Motivación del estudio 12

1.2 Organización de la tesis y metodología 13

$\begin{array}{ll}1.3 \text { Zona de estudio } & 14\end{array}$

1.4 Marco geológico y tectónico 15

1.4.1 Sistema de fallas Magallanes - Fagnano 15

$\begin{array}{lr}1.5 \text { Sismicidad histórica } & 19\end{array}$

1.6 Estaciones sismológicas 19

$\begin{array}{ll}1.7 \text { Sismicidad actual } & 22\end{array}$

$\begin{array}{ll}1.8 \text { Otros antecedentes } & 24\end{array}$

\section{CAPÍTULO II: Funciones del Receptor: Teoría y Aplicación}

2.1 Introducción $\quad 27$

2.2 ¿Qué es una Función del Receptor? $\quad 28$

2.3 Descripción matemática 31

2.3.1 Filtro Gaussiano $\quad 34$

2.3.2 Ejemplo con tres arribos $\quad 35$

2.3.3 Deconvolución iterativa en el dominio del tiempo 37

2.4 Aplicación de la técnica en TdF 37

$\begin{array}{ll}2.4 .1 \text { Selección de datos } & 38\end{array}$

2.4.2 Preprocesamiento de los datos 39

2.4.2.1 Conversión del formato y preparación del header 39

2.4.2.2 Reducción de la media y de la pendiente $\quad 40$

2.4.2.3 Rotación de las componentes $\quad 40$

2.4.2.4 Filtro pasabanda $\quad 41$

2.4.3 Cálculo de las FRs $\quad 43$

2.5 Resultados $\quad 43$

2.5.1 Estación TRVA $\quad 44$

2.5.2 Estación USHA

2.5.3 Estación DSPA $\quad 46$

2.5.4 Estación ELCA $\quad 47$

2.5.4.1 Heterogeneidades en la estación USHA 48

\section{CAPÍTULO III: Apilamiento de las Funciones del Receptor: Método de H-k stacking}

3.1 Estimación del espesor cortical y de la relación $V_{\mathrm{P}} / \mathrm{V}_{\mathrm{S}}$

$\begin{array}{ll}3.2 \text { Apilamiento de las FRs } & 54\end{array}$

3.2.1 Método de H-k stacking $\quad 55$

$\begin{array}{ll}\text { 3.2.1.1 Selección de factores de peso } \mathrm{w}_{\mathrm{i}} & 57\end{array}$

3.2.1.2 Estimación de la incertidumbre $\quad 58$

3.3 Aplicación del método en TdF 59

3.3.1 Procedimiento $\quad 59$ 
CAPÍTULO IV: Inversión no-lineal de Funciones del Receptor: Método Neighbourhood Algorithm

4.1 Introducción 65

4.2 Método de Neighbourhood Algorithm (NA) 66

4.2.1 Celdas de Voronoi $\quad 67$

4.2.2 Ventajas del NA $\quad 70$

4.3 Inversión no-lineal de FRs mediante el algoritmo NA en TdF 71

4.3.1 Definición del modelo inicial $\quad 71$

4.3.2 Parámetros de control $\quad 72$

$\begin{array}{ll}\text { 4.3.3 Selección de datos } & 72\end{array}$

4.3.4 Resultados $\quad 73$

$\begin{array}{ll}\text { 4.3.4.1 Estación TRVA } & 73\end{array}$

4.3.4.2 Estación USHA $\quad 76$

4.3.4.3 Estación DSPA $\quad 78$

4.3.4.4 Estación ELCA $\quad 80$

4.3.5 Inversión simultánea de FRs $\quad 81$

$\begin{array}{ll}4.4 \text { Discusión y conclusiones } & 84\end{array}$

CAPÍTULO V: Interferometría sísmica: Correlaciones cruzadas de ruido sísmico ambiental

5.1 Introducción $\quad 86$

5.1.1 Definición de ruido sísmico $\quad 87$

$\begin{array}{ll}5.1 .2 \text { Tipos de ruido } & 88\end{array}$

$\begin{array}{ll}\text { 5.1.3 Contenido espectral } & 88\end{array}$

5.1.4 Microsismo primario y secundario $\quad 91$

5.1.5 Fundamentos 93

5.1.5.1 Correlación cruzada 93

5.1.5.2 ¿Qué es la función de Green? 96

5.2 Aplicación de la técnica en TdF 98

5.2.1 Preprocesamiento de los datos 98

5.2.2 Cálculo de las correlaciones cruzadas $\quad 99$

5.2.3 Apilamiento de las correlaciones cruzadas $\quad 100$

$\begin{array}{ll}5.3 \text { Resultados y discusión } & 102\end{array}$

CAPÍTULO VI: Obtención de curvas de dispersión a partir de ruido sísmico: Cálculo e inversión

6.1 Introducción 106

6.1.1 Ondas Rayleigh y velocidades de dispersión 107

6.2 Cálculo de las curvas de dispersión 108

6.2.1 Efecto de la longitud del apilamiento y de la época estacional del año 111

6.3 Inversión de las velocidades de grupo 114

6.3.1 Resultados 117

$\begin{array}{ll}\text { 6.3.2 Discusión y conclusiones } & 120\end{array}$

CAPÍTULO VII: Resultados y conclusiones generales 121

$\begin{array}{ll}\text { Apéndice A } & 128\end{array}$

Apéndice B 132

$\begin{array}{ll}\text { Referencias } & 136\end{array}$ 


\section{Introducción general}

\subsection{Motivación del estudio}

La Isla Grande de Tierra del Fuego (TdF) (Figura 1.1) presenta un escenario geotectónico diverso y se sitúa en una zona de actividad tectónica, una de cuyas manifestaciones es la actividad sísmica la cual se traduce en la producción de terremotos. Esta región es uno de los pocos lugares de la Tierra donde se pueden observar las características geológicas superficiales y subsuperficiales relacionadas con un límite de placas transformante continental, pero es una de las regiones menos conocidas o estudiadas debido a su lejanía y las dificultades de acceso en gran parte de su territorio.

Si bien la sismicidad más reciente de la región está caracterizada por sismos de baja a mediana magnitud, la Isla posee una sismicidad histórica que incluye dos eventos de carácter destructivo ocurridos el 17 de diciembre de 1949 con magnitudes 7.5 y 7.8 y epicentros $69.0^{\circ} \mathrm{O} 54.2^{\circ} \mathrm{S}$ y $69.2^{\circ}$ O 53.9 S, respectivamente (Jaschek et al., 1982). Si se considera un sismo con epicentro ubicado sobre la falla de Magallanes-Fagnano perpendicularmente a Ushuaia, la distancia epicentral sería de $28 \mathrm{~km}$. En este caso hipotético habría que esperar para la zona de Ushuaia una intensidad de IX en la Escala de Mercalli Modificada (Coronato et al., 1995). Si ocurriese un sismo similar con epicentro cercano a la ciudad de Tolhuin, generaría un gran impacto en la actualidad ya que afectaría al gasoducto que pasa por esa zona. Además la ruta 3, única conexión entre la ciudad de Ushuaia y el resto de las ciudades, se vería afectada dejando a Ushuaia aislada sin energía eléctrica ni calefacción.

En una zona sísmicamente activa es importante estar preparados ante la ocurrencia de terremotos y poder evaluar un plan de contingencia. Para llevar a cabo esto, es fundamental conocer con precisión los sistemas de fallas y campos de estrés de la región. Desde este punto de vista, determinar la estructura cortical es esencial para poder realizar la localización de los eventos sísmicos con el menor error posible y así determinar las zonas con mayor probabilidad de ocurrencia de terremotos. Ésto fue lo que motivó al presente trabajo de Tesis, cuyo principal objetivo fue el de estimar la profundidad de la discontinuidad de Mohorovicic (Moho), la relación de velocidades sísmicas y modelos de velocidad de onda S con el fin de aportar al conocimiento de la estructura cortical en TdF. 


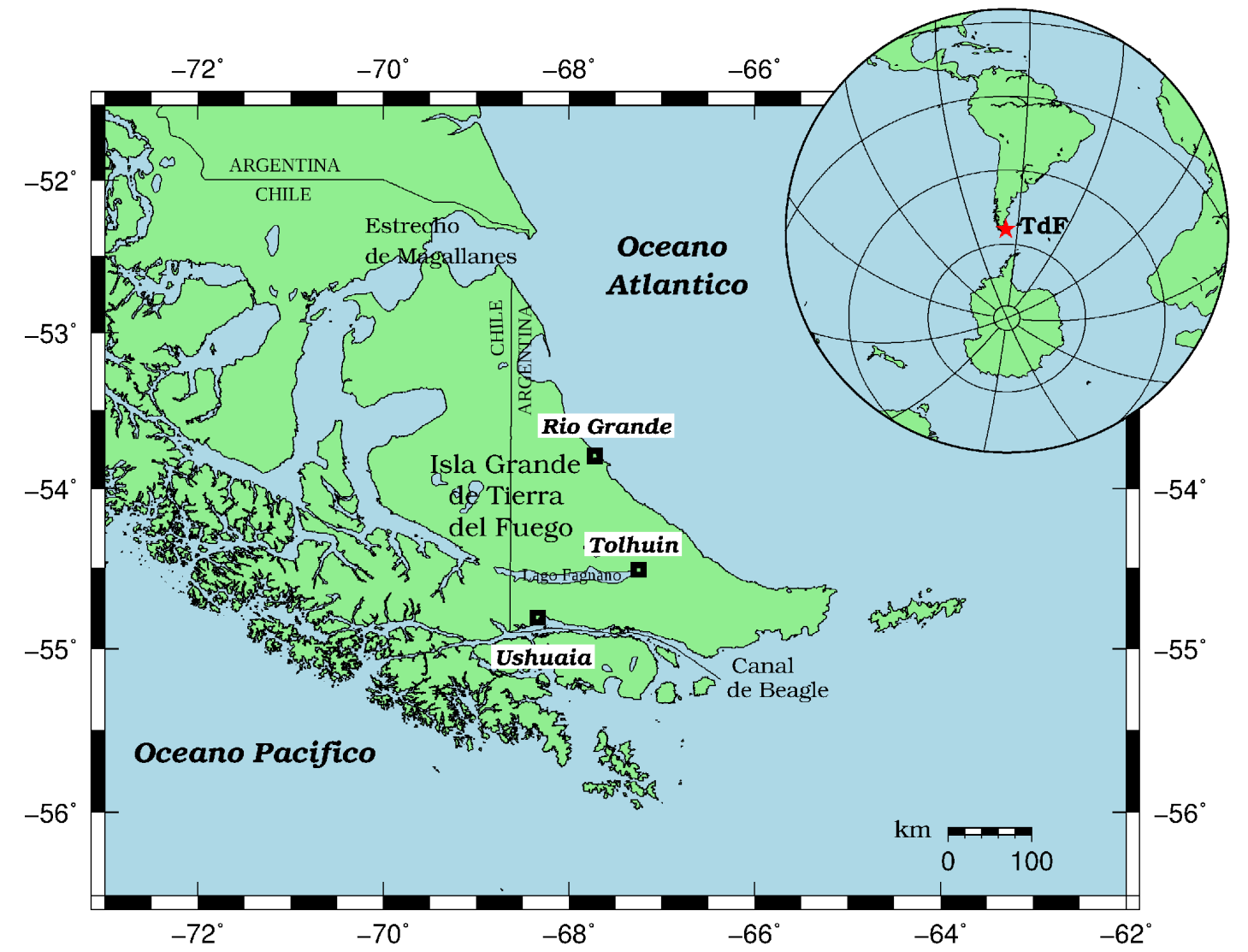

Figura 1.1: Ubicación geográfica de TdF. Río Grande, Tolhuin y Ushuaia son las ciudades principales de la Isla en el sector Argentino.

\subsection{Organización de la tesis y metodología}

La Tesis se compone de siete capítulos. En este primer capítulo se presenta una introducción general donde se describe la zona de estudio, sus características geológicas, tectónicas y sísmicas así como también los estudios previos realizados en la zona mediante otras metodologías. Estos temas son fundamentales para comprender la motivación que dió lugar a este trabajo. Los capítulos dos, tres, cuatro, cinco y seis desarrollan las diferentes metodologías utilizadas para estimar la estructura de la corteza. La primera metodología implementada incluye la técnica del cálculo, apilamiento (stack) e inversión de las Funciones del Receptor (FR). Por otro lado, con el fin de complementar los resultados obtenidos con esta primera técnica, se aplicó el método de la interferometría sísmica a través del cálculo de correlaciones cruzadas de ruido sísmico ambiental. A partir de esta última técnica, se calcularon curvas de dispersión lo cual aportó información sobre la estructura más superficial de la corteza. Finalmente en el último capítulo se analizan los resultados obtenidos por los diferentes métodos y se mencionan las respectivas conclusiones. 


\subsection{Zona de estudio}

Tierra del Fuego es el nombre del Archipiélago compuesto por un grupo de islotes e islas menores que se encuentran ubicadas al sur del Estrecho de Magallanes (Figura 1.1). La Isla Grande de Tierra del Fuego pertenece a este grupo de islas siendo la más grande de Sudamérica y constituye la zona de estudio de la presente Tesis. Alcanza una superficie de aproximadamente $45000 \mathrm{~km}^{2}$ y se divide en dos partes a través del meridiano de $68^{\circ} 36^{\prime} \mathrm{O}$ que corresponde a la frontera internacional: la región oriental corresponde a territorio argentino mientras que la región occidental a territorio chileno. TdF limita con el canal de Beagle al sur, con el Estrecho de Magallanes al norte, con el océano Atlántico al este y con el océano Pacífico al oeste. El sector argentino de la Isla corresponde a la provincia de Tierra del Fuego, Antártida e islas del Atlántico Sur, siendo Ushuaia la capital.



Figura 1.2: Mapa geológico simplificado del sector argentino de TdF adaptado de Tassone et al. (2005). BCS:

Falla del Canal de Beagle, FMF: Falla Magallanes - Fagnano. 1. Basamento (Paleozoico - Jurásico), 2.

Riolitas, basaltos, conglomerados y turbiditas del Jurásico Superior, 3. Depósitos del Cretácico Inferior, 4.

Depósitos del Cretácico Superior, 5. Depósitos deformados del Terciario y 6. Depósitos no deformados del

Terciario. DSPA, ELCA, TRVA y USHA son las estaciones sismológicas utilizadas en el estudio. Las distintas unidades y formaciones geológicas fueron determinadas por Caminos y Nullo (1979), Caminos et al. (1981), Olivero y Martinioni (2001) y Ghiglione (2003) 


\subsection{Marco geológico y tectónico}

El aspecto geológico y tectónico de TdF es el resultado de la evolución del extremo sur de Sudamérica asociado con el ciclo orogénico andino del Mesozoico-Cenozoico que involucró la interacción entre las placas Sudamericana, Antártica y de Scotia (Figura 1.2). La evolución tectónica de esta zona se puede resumir con tres episodios tectónicos: un régimen extensivo durante el Jurásico tardío-Cretácico temprano, un período de compresión durante el Cretácico-Paleoceno y finalmente un episodio de movimiento transformante (strike-slip) a partir del Oligoceno (Menichetti et al., 2008). La evolución tectónica más reciente de Sudamérica está relacionada con los movimientos de la placa de Scotia que trasladó fragmentos continentales a más de $1000 \mathrm{~km}$ de distancia horizontal de TdF (Dalziel et al., 1975).

\subsubsection{Sistema de fallas Magallanes - Fagnano}

El límite entre la placa Sudamericana y la de Scotia define un sistema de fallas que se conoce como Sistema de fallas Magallanes-Fagnano (SFMF). Este sistema está representado por una falla transformante que corre desde la parte oeste del norte de la dorsal de Scotia hacia la fosa de Chile a aproximadamente $50^{\circ}$ S (Fuenzalida, 1972; Dalziel y Brown, 1989). Algunos autores asignan 30 Ma de edad a este sistema, correspondiendo con los estadíos tempranos del desarrollo del oeste del Mar de Scotia (Barker y Burell, 1977). Otros asignan 100 Ma asociado con el movimiento activo entre Sudamérica y la Península Antártica (de Wit, 1977; Grunow et al., 1991, 1992). TorresCarbonnel et al. (2008) estimó que la edad máxima de inicio de transcurrencia es de $\sim 7 \mathrm{Ma}$, coincidiendo con el nacimiento del límite constructivo entre las placas Sandwich y Scotia (Figura 1.3). Este sistema representa el borde de contacto entre las placas Sudamericana y de Scotia y está asociado a una falla continental transformante (Olivero y Martinioni, 2001). En la región de los Andes Fueguinos (Figura 1.3) se encontraron datos que evidencian que el régimen de desplazamiento de rumbo a través del SFMF ha estado activo desde al menos el Eoceno tardío (Olivero y Martinioni, 2001), lo que indica la presencia de las fallas de rumbo en la zona.

La traza principal del SFMF se conoce como Falla de Magallanes-Fagnano (FMF) y pertenece a un grupo de fallas de rumbo subparalelas. La FMF recorre la Isla de este a oeste en la latitud del lago Fagnano y es una falla activa. Tiene un movimiento promedio de unos pocos milímetros por año (Del Cogliano et al., 2000; Smalley et al., 2003; Mendoza et al., 2011, 2015). El modelo más aceptado es el de una falla transformante vertical de dos dimensiones, que estaría soldada a unos 15 km de profundidad (Smalley et al., 2003). Las evidencias geológicas y la orientación de las trazas sugieren que la falla principal es paralela al eje mayor del lago Fagnano (Klepeis, 1994). La Isla 
está dividida en dos bloques continentales como consecuencia de esta falla principal. La región de la Isla ubicada al sur del lago pertenece a la placa de Scotia, la cual se mueve relativamente hacia el este respecto a la placa Sudamericana. Dicha región ofrece un ambiente geológico caracterizado por la Cordillera Fueguina, la cual presenta un rumbo este-oeste como resultado del movimiento transformante entre las placas Sudamericana, la Antártica y la de Scotia. Este movimiento se contrasta con el rumbo norte-sur que muestra la Cordillera de los andes en el resto de Sudamérica. La Cordillera Fueguina está constituida por un zócalo leptometamórfico atribuido al Paleozoico y sobre éste se apoyan rocas volcánicas ácidas que corresponden al Jurásico Medio a Superior. Estas rocas están cubiertas por depósitos de edad cretácica superior y terciaria. Por otro lado, la región ubicada al norte pertenece a la placa Sudamericana, la cual se desplaza relativamente hacia el oeste respecto a la placa de Scotia y presenta un ambiente geológico extra-andino estable. Desde el punto de vista tectónico, estos dos bloques presentan diferentes características: la parte sur de TdF presenta una fuerte evidencia morfológica de actividad cuaternaria relacionada con un régimen de rumbo sinestral E-O, mientras que la zona norte está afectada por una tectónica de extensión asociada con sistemas de fallas normales hacia el este del Estrecho de Magallanes (Menichetti et al., 2008).



Figura 1.3: Ubicación geográfica de las placas Sudamericana, Antártica, Scotia y Sandwich (Imagen extraída y modificada de Olivero y Martinioni, 2001).

Otras fallas pertenecientes al SFMF son: el canal de Beagle, Lasifashaj, Deseado, Carbajal Valley y Río Turbio (Figura 1.4) (Klepeis, 1994; Menichetti et al., 2008). Algunas de estas fallas de rumbo tienen una importante componente extensional asociadas con fallas normales con inclinación hacia el norte, en la zona de Termas (indicada con la presencia de la estación sismólogica TRVA en la Figura 1.4) y Paso Garibaldi (ubicado en las cercanías de la ciudad de Ushuaia), ambas en la zona sur de TdF (Menichetti et al., 2008). Por otro lado se han identificado varias estructuras de extensión caracterizadas por fallas normales con rumbo NO-SE e 




Figura 1.4: Modelo de elevación digital y ubicación de las fallas subsidiarias que comprenden el SFMF. Las estrellas muestran la ubicación de tres de las estaciones utilizadas en este trabajo: ELCA, TRVA y USHA. Los números indican algundas de las principales fallas que componen el SFMS: (1). Lago Deseado, (2). Catamarca, (3). S. Rafael, (4). V. Carbajal, (5). Andorra, (6). Canal de Beagle, (7). V. Lasifashaj, (8) S. Las Pinturas, (9). Knokeke y (10). Río Turbio. (Imagen extraída y modificada de Menichetti et al., 2008).

En el sector centro-este de la Isla, las fallas han sido identificadas principalmente a partir de análisis de imágenes (remote-sensing images). Estos segmentos de fallas han sido localizados en los valles de ríos y generalmente están asociados con mínimos locales de gravedad (Tassone et al., 2005). Velasco et al. (2002) han especulado que la ubicación de las fallas transformantes activas ha migrado hacia el norte y que la actividad tectónica actual se concentra en la falla principal del SFMF y en las fallas subsidiarias hacia el norte. Por otra parte, un estudio paleosísmico realizado en las planicies de la costa Atlántica de la Patagonia revela la presencia de dos fallas cuya fuente ha sido directamente relacionada con la FMF (Bonorino et al., 2012).

La presencia de varias fallas con rumbo N-S que atraviesan la traza principal del SFMF y la limitada información geológica y geofísica de esta zona siguen sin poder permitir la exacta ubicación de algunas fallas activas (Lodolo et al., 2003; Tassone et al., 2005; Menichetti et al., 2008). Sin embargo, a partir de estudios geológicos y geofísicos realizados en algunos sectores de la Isla se ha podido comprobar la presencia de diversas fallas que caracterizan el ambiente geotectónico de la zona. Un ejemplo de esto es la sección geológica obtenida por Tassone et al. (2005) a partir de un modelo numérico de datos gravimétricos (Figura 1.5) donde se observan las diversas estructuras geológicas alrededor de la falla principal del SFMF. 




Figura 1.5: a) Mapa geológico de la zona este del Lago Fagnano realizado en un estudio previo por Tassone et al. (2005). (1). Depósitos glaciolacustres y lacustres del cuaternario, (2). Secuencias glaciolacustres del pleistoceno, (3a). Formación Río Claro, (3b). Grupo La Despedida compuesto por areniscas del terciario, (4). Equivalente a Co. Matrero, (5). Intrusivo Hewhoepen, (6). Formación Yahgan, (7). Clases de inclinación: (a) $0^{\circ}-30^{\circ}$, (b) $30^{\circ}-45^{\circ}$, (c) $45^{\circ}-60^{\circ}$ y (d) $60^{\circ}-85^{\circ}$, (8) Falla inversa, (9) Falla normal, (10) Falla transcurrente y (11) Lineamientos tectónicos inferidos. b) Sección geológica interpretada (Perfil AB del mapa geológico anterior) a partir de un modelo numérico obtenido con datos gravimétricos y combinados con datos litológicos y estructurales obtenidos en el campo realizado realizado en un estudio previo. Los triángulos negros indican la ubicación de las estaciones magnéticas y gravimétricas. L.F indica el Lago Fagnano. Los números a la derecha de las referencias indican las densidades de cada unidad geológica expresadas en $\mathrm{g} / \mathrm{cm}^{3}$. (Imágenes extraídas y modificadas de Tassone et al., 2005). 


\subsection{Sismicidad histórica}

Fernando de Magallanes fue uno de los primeros europeos en llegar a estas tierras en $1620 \mathrm{y}$ fue quien le puso el nombre de Tierra del Fuego. La ciudad de Ushuaia fue fundada en 1868 y se convirtió oficialmente en una ciudad en 1884. En 1895 la población registrada era de 480 habitantes en toda la Isla. Si bien la población era escasa, los antecedentes de actividad sísmica de esta zona se remontan al año 1879 (Lomnitz, 1970). El 2 de febrero de 1879 a las 3 hrs. 30 minutos, hora local, hubo un terremoto de magnitud estimada entre 7 y 7.8 que afectó a toda la región. Este sismo alcanzó una intensidad de 7 en Punta Arenas (Chile), y de 8 en Tierra del Fuego (Argentina).

El 17 de diciembre de 1949 ocurrieron dos eventos de magnitudes 7.5 y 7.8 (Jaschek et al., 1982), con sus precursores y réplicas. Se conocen reportes de testimonios de los habitantes acerca de estos eventos de gran magnitud (Buffoni, 2008). Si bien los daños no fueron económicamente importantes porque las casas estaban construidas de madera y los servicios públicos eran muy simples, es importante considerar el impacto que hoy tendría en la zona un evento de tal magnitud. Al día siguiente se registraron 24 réplicas. Grandes olas y corrientes marinas anormales fueron observadas en Porvenir y en el seno Almirantazgo. Derrumbes asociados al terremoto fueron observados en la costa occidental de Tierra del Fuego y a lo largo de las bancadas del lago Fagnano, y produjeron tres muertes en la costa de la bahía de San Nicolás. Algunos autores han asignado una magnitud de 7.5 al evento ocurrido en 1949 (Lomnitz, 1970).

Jaschek et al., 1982 han relocalizado 1200 epicentros de sismos argentinos ocurridos entre 1920 y 1963. Además de un análisis de sismicidad histórica se conocen reportes de sismos en los años 1929, 1930, 1944, 1949, 1970 y precursores y réplicas del evento de gran magnitud ocurrido el 17 de diciembre de 1949 (Sabbione et al., 2007a).

\subsection{Estaciones sismológicas}

La instalación de estaciones sismológicas en TdF tiene sus inicios en el año 1995. La primera estación sismológica instalada se denominó USHU por su cercanía a la ciudad de Ushuaia (IAAPNRA). Dicha estación fue equipada inicialmente con un sensor Güralp CMG-3T y posteriormente fue reemplazado por un sensor Güralp 40T. Datos provenientes de dicha estación fueron analizados obteniendo como resultado la localización de 41 sismos mediante técnicas que utilizan datos de una sola estación (Febrer et al., 2000). USHU funcionó hasta octubre de 2005 y perteneció a la red Antartic Seismology Argentine Italian Network (ASAIN). La red de estaciones sismológicas argentino-italiana de banda ancha ASAIN funciona desde 2001 y forma parte de convenios entre instituciones argentinas: el Instituto Antártico Argentino y la Universidad Nacional de La Plata (UNLP) con el Istituto Nazionale di Oceanografia e Geofisica Sperimentale (OGS) de 
Trieste, Italia. En junio de 1999, en el marco de un proyecto para el estudio de la geofísica y geodinámica de la Isla de Tierra del Fuego, se instaló la estación sismológica DSPA (Despedida, UNLP-PNRA). Dicha estación se encuentra ubicada en la Estancia Despedida, $50 \mathrm{~km}$ al sudoeste de la ciudad de Río Grande. Inicialmente estuvo equipada con un sensor Lennartz LE-3Dlite de 1 $\mathrm{Hz}$, un adquisidor de tres canales con Conversor Analógico Digital de 16 bits con registro continuo a 25 mps y una PC para la operación, control y almacenamiento de los datos. El primer evento registrado por DSPA ocurrió el 9 de junio de 1999 y correspondió a un sismo cuyo epicentro se localizó a 750 km al este de las Islas Malvinas. En noviembre de 2002, la estación DSPA debió ser ampliada para alojar un nuevo sensor de banda a ancha Güralp 3T el cual fue provisto por el OGS. Registros sísmicos obtenidos en dicha estación fueron analizados desde junio de 1999 hasta marzo del 2002 y se localizaron más de 60 eventos que fueron correlacionados con el ambiente tectónico de Tierra del Fuego y zonas aledañas (Plascencia et al., 2002). En enero de 2003 el sensor Lennartz LE-3Dlite, que había sido previamente instalado en la estación DSPA, fue instalado en la zona de Termas del Río Valdez constituyendo la tercera estación sismológica con el nombre de TRVA, donde funcionó por unos tres meses. Posteriormente dicho sensor fue trasladado a Río Grande e Isla de los Estados (noviembre 2003 a enero 2004) generando estaciones temporarias, como es el caso de la estación IDEA instalada en la Isla de los Estados. En febrero de 2004 volvió a ser instalado en Termas. En el año 2005 fue reemplazado por el sensor Güralp 40T proveniente de la estación USHU, y en febrero de 2006 se dotó a la estación TRVA de otro Güralp 3T provisto por el OGS. El 19 de octubre del 2006 se instaló una nueva estación en la Bahía El Torito a orillas de lago Fagnano denominada BETA. Esta estación tenía instrumental de corto período, equipada con un sensor Lennartz 3D-lite que funcionó hasta febrero de 2011. A partir de noviembre de 2011 BETA fue equipada con el sensor Güralp 40T hasta la fecha. El sensor Lennartz fue instalado temporalmente en una nueva estación denominada SARA, desde junio a diciembre de 2012 con el fin de registrar la sismicidad local. El 12 de octubre de 2007 se instaló la estación sismológica ELCA situada en la estancia Los Cerros. Dicha estación estaba equipada con un sensor Güralp 40T y funcionó hasta abril de 2011. Actualmente las estaciones que se encuentran en funcionamiento y cuya operación y logística está a cargo del personal de la Estación Astronómica de Río Grande (EARG) son: DSPA, TRVA y BETA. Además en la Isla funciona una estación sismológica permanente denominada USHA perteneciente a la red Preparatory Comission for the Comprehensive nuclear-tet-ban treaty Organization (CTBTO) y cuyo responsable local es el Instituto Nacional de Prevención Sísmica (INPRES). Mediante un convenio firmado entre la UNLP y el INPRES, se disponen de datos de esta ultima estación. En la presente Tesis se utilizaron los datos de las estaciones banda ancha DSPA, TRVA, ELCA y USHA por ser las que presentaron mayor calidad y cantidad de datos disponibles (Figuras 1.6-1.9 y Tabla 1.1). 


\begin{tabular}{|c|c|c|c|c|}
\hline Estación & Latitud ( $\left.{ }^{\circ} \mathrm{S}\right)$ & Longitud $\left({ }^{\circ} \mathrm{O}\right)$ & Sensor & Operador \\
\hline USHU & 54.85 & 68.55 & Guralp CMG-3T & EARG \\
\hline DSPA & 53.95 & 68.26 & Guralp CMG-3T & EARG \\
\hline TRVA & 54.68 & 67.34 & Guralp CMG-3T & EARG \\
\hline BETA & 54.60 & 68.20 & Lennartz LE-3Dlite & EARG \\
\hline ELCA & 54.34 & 67.85 & Guralp CMG-40T & EARG \\
\hline USHA & 54.81 & 68.43 & Streckeisen STS-2 & INPRES \\
\hline SARA & 53.43 & 68.18 & Lennartz LE-3Dlite & EARG \\
\hline IDEA & 54.80 & 64.36 & Lennartz LE-3Dlite & EARG \\
\hline
\end{tabular}

Tabla 1.1: Estaciones sismológicas en TdF. Actualmente las estaciones que están en funcionamiento son TRVA, DSPA, BETA y USHA.


Figura 1.6: Estación USHA ubicada en las cercanías de la ciudad de Ushuaia y administrada por el INPRES. Izq.:vista de la estación. Der.: albergue del primer sensor con la cual estuvo equipada la estación en sus inicios. Actualmente dicha estación está equipada con un sensor STS-2.
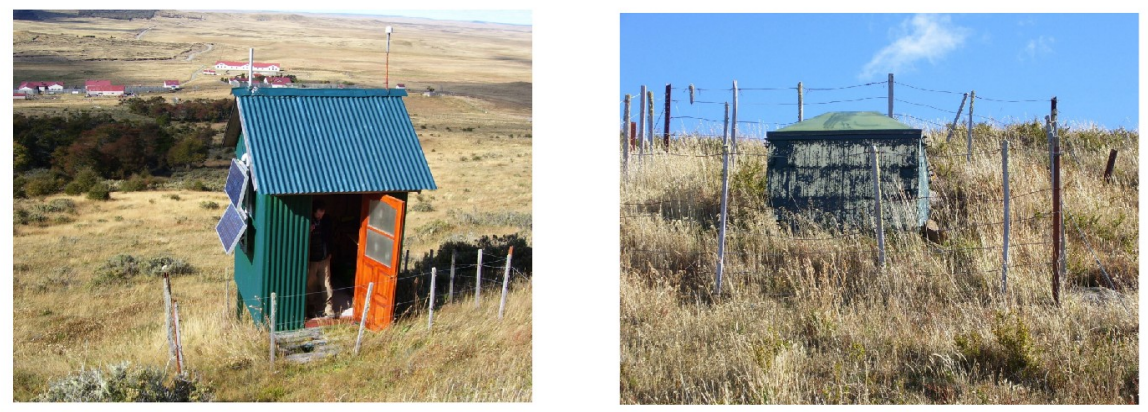

Figura 1.7: Estación DSPA ubicada en la estancia Despedida. Izq.: albergue del sistema de adquisición, almacenamiento y provisión de energía. Der.: albergue del sensor. 

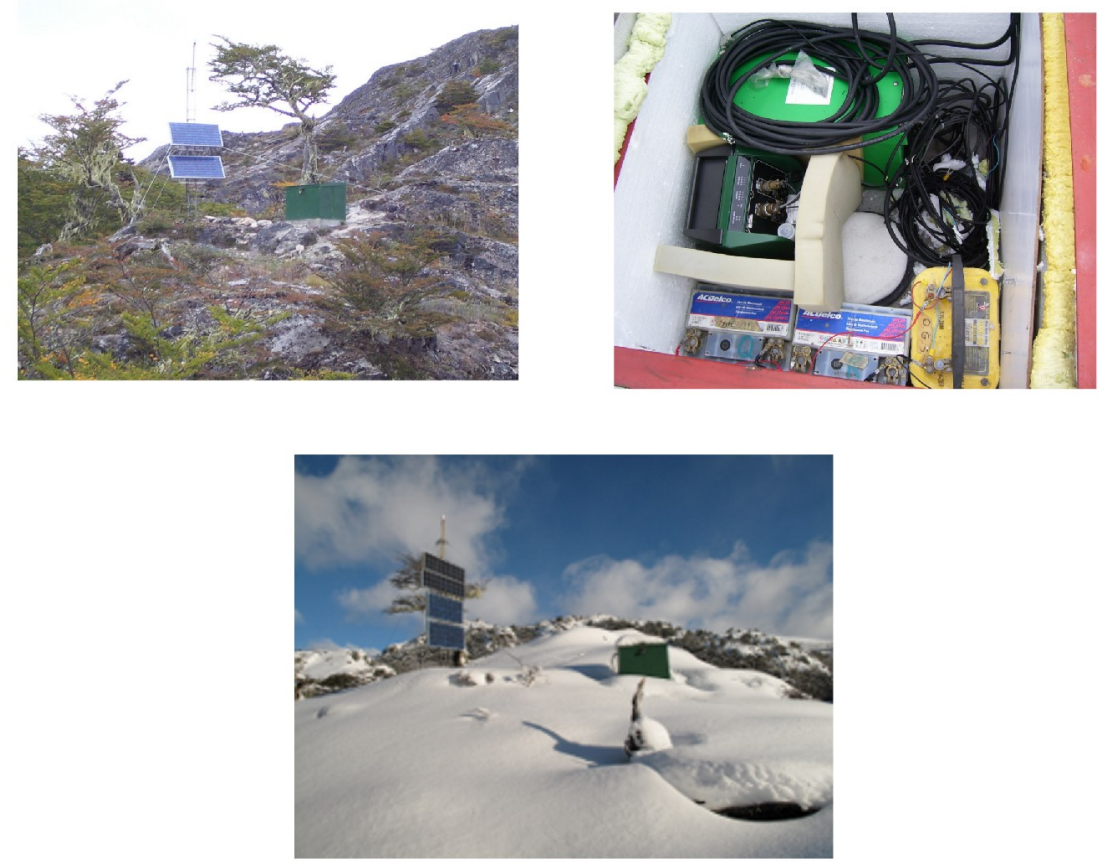

Figura 1.8: Estación TRVA. Izq.: vista panorámica de la estación. Der.: detalle del albergue del sensor. Se observa además una imagen de la estación durante los meses de invierno.


Figura 1.9: Estación ELCA. Izq.: ubicación del sensor. Der.: vista de la estación.

\subsection{Sismicidad actual}

La instrumentación en TdF se inició en la década del 90 con una sola estación. Desde este punto de vista, obtener precisiones hipocentrales con mínimo error ha sido uno de los problemas a resolver. Por otra parte, la instalación y mantenimiento de las estaciones resultan muy complicados dada la accesibilidad, la topografía y las condiciones meteorológicas que se presentan, principalmente durante los meses de invierno. Con las primeras estaciones sismológicas instaladas 
en la Isla, comenzaron a registrarse sismos de baja a mediana magnitud. A pesar que es notorio el escaso número de estaciones sismológicas de redes globales por la ubicación geográfica de la región, a medida que se avanzó en la instalación de nuevas estaciones ha sido posible mejorar la calidad de la localización de los eventos. El hecho de que la sismicidad registrada sea de baja y mediana magnitud, indica la importancia de contar con una red sismológica local, ya que escasos sismos fueron registrados por centros internacionales de cálculo de hipocentros.

Febrer et al. (2000) fue uno de los primeros autores en analizar los registros sísmicos de las primeras estaciones sismológicas instaladas en la Isla. En su trabajo como en los que le siguieron otros autores (Plascencia et al., 2002; Sabbione et al., 2007a, 2007b; Buffoni et al., 2009) se ha realizado la localización de eventos de baja a mediana magnitud que han sido correlacionados con el ambiente tectónico que caracteriza a esta región. El estudio de sismicidad realizado por Febrer et al. (2000) tiende a confirmar que además de los grandes sismos históricos publicados entre otros, en Jaschek et al. (1982), existe una sismicidad difusa de baja magnitud en torno a la falla FMF. Por otro lado existen evidencias que indican la presencia de por lo menos una agrupación de epicentros, de magntitud menor que 3.5, asociados con la Cordillera de Darwin, estructura que pudo haber actuado como una visagra estructural durante la evolución oroclinal de los Andes PatagónicoFueguinos (Febrer, 2002).

Sabbione et al. (2007a) presentaron el primer catálogo de sismicidad de la región (Figura 1.10).

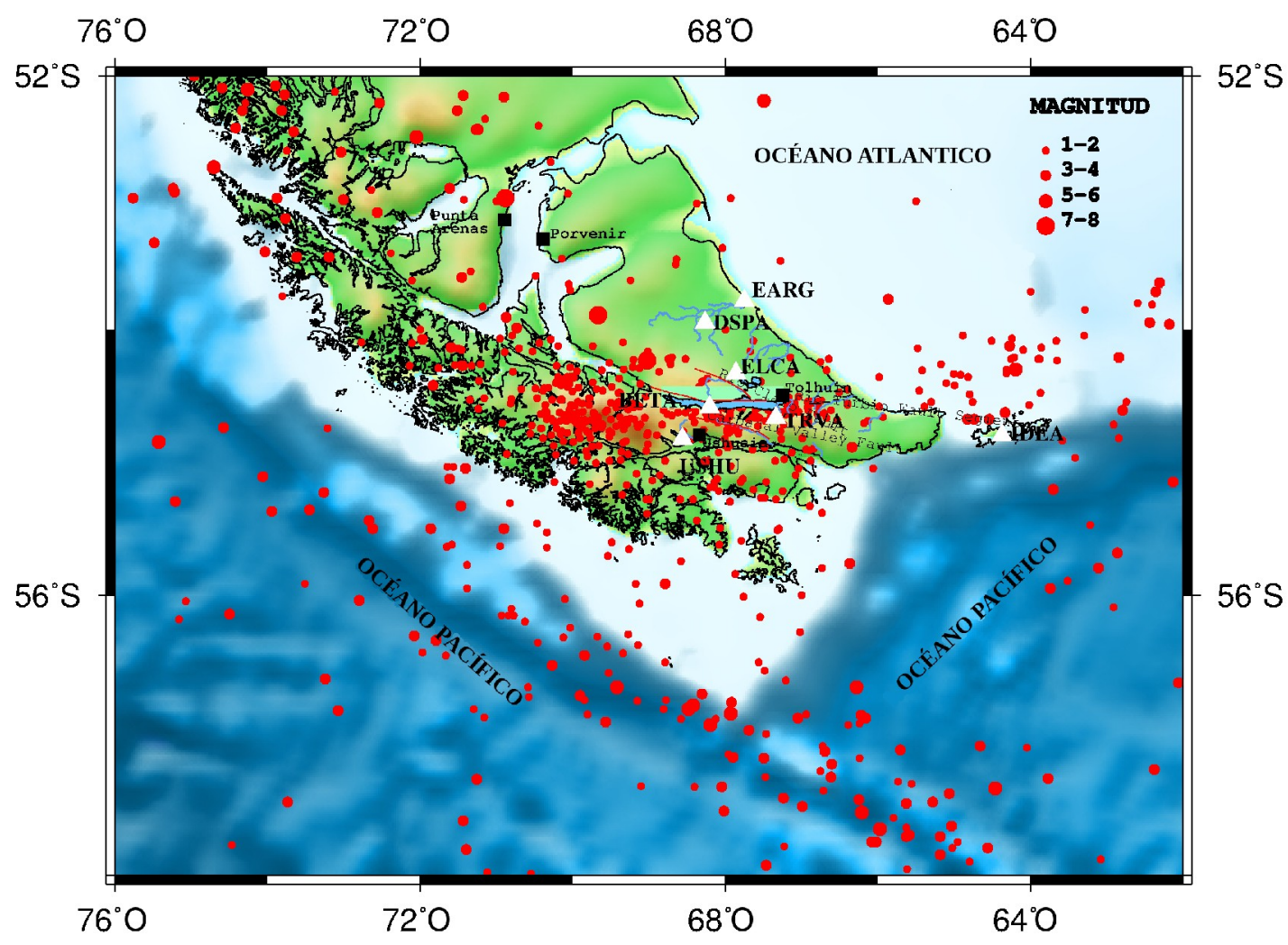

Figura 1.10: Catálogo de sismicidad de TdF (Imagen extraída y modificada de Sabbione et al., 2007a). 
El mismo fue confeccionado a partir de datos recolectados por centros nacionales, regionales e internacionales (INPRES, CERESIS, ISC, USGS), y de la sismicidad registrada por las estaciones de TdF. Durante el período junio 1999 - julio 2007, la red local de estaciones registró alrededor de 750 eventos de magnitudes inferiores a 4.4. Dicho catálogo reúne la sismicidad histórica de la zona y la registrada hasta julio de 2007, mostrando una concordancia con el ambiente geotectónico de la región.

Por otro lado, en la Figura 1.11 se observa la localización de eventos registrados por las estaciones de TdF durante 1999 y 2015. Esto evidencia la continua liberación de energía que existe en la zona asociada con el movimiento relativo entre las placas de Scotia y Sudamericana.

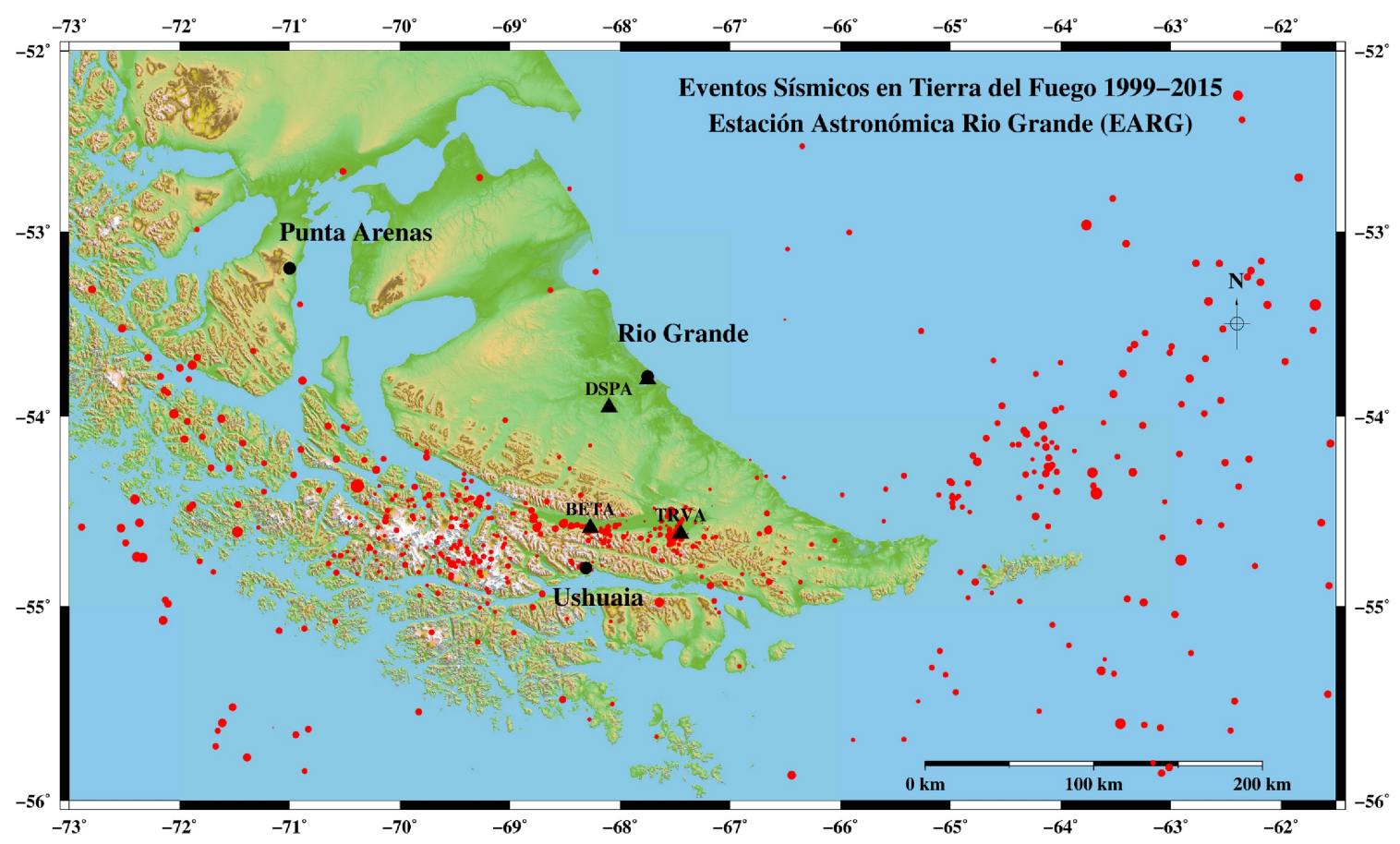

Figura 1.11: Localización de eventos sísmicos en TdF. DSPA, BETA, TRVA son las estaciones sismológicas que se encuentran en funcionamiento en la actualidad dependientes de EARG. (Mapa provisto por EARG).

\subsection{Otros antecedentes}

En los incisos anteriores ya se ha mencionado el motivo por el cual TdF representa un área clave en la investigación de la geodinámica involucrada en la interacción de las placas Sudamericana y de Scotia, siendo de particular interés ya que en esta zona se encuentra el único segmento sobre tierra que existe entre el contacto de dichas placas. Una manera de estudiar las 
zonas sísmicamente activas consiste en realizar un análisis de deformación de las componentes de velocidad horizontal de la zona. En 1993 comenzaron las observaciones geodésicas en la zona con el fin de estudiar el movimiento relativo a través de la FMF. Desde entonces se ha constituido una red regional a partir de la cual es posible estudiar el proceso cinemático que está teniendo lugar en la Isla. Los primeros resultados mostraron velocidades de $5 \mathrm{~mm}$ por año (Del Cogliano et al., 2000) y de 6.6 +- $1.3 \mathrm{~mm}$ por año (Smalley et al., 2003). Mendoza et al. (2011) determinaron un cinturón de deformación de 30 km de ancho situado en la traza principal del sistema de fallas, el cual se mueve horizontalmente con una velocidad promedio relativa de $4.4 \pm 0.6$ (este) y $-0.3 \pm$ 0.4 (norte) mm por año (Figura 1.12).

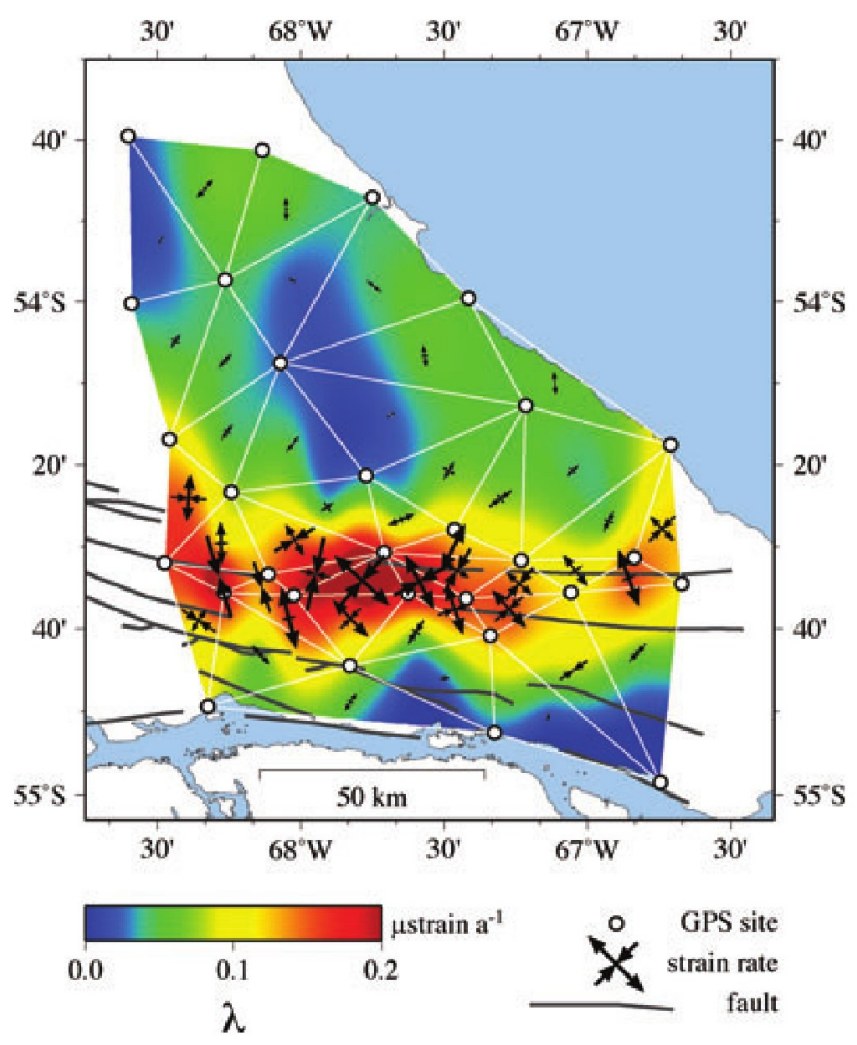

Figura 1.12: Componentes principales de la tasa de deformación dibujados como vectores de acortamiento o extensión. (Imagen extraída de Mendoza et al., 2011).

Estudios más recientes (Mendoza et al., 2015) indican que el movimiento a través del SFMF es de $5.9 \pm 0.2 \mathrm{~mm}$ por año (Figura 1.13). Esto es una clara evidencia de las zonas con mayor actividad de deformación y del movimiento que se está llevando a cabo a lo largo de la zona del SFMF. El campo de velocidades de deformación obtenido demuestra que la región del sector oriental de TdF puede ser dividida en dos zonas separadas por la cuenca del lago Fagnano. El 
promedio de las velocidades horizontales en ambas zonas es similar pero existe una diferencia en la dirección del movimiento en cada una de ellas. La mayor deformación se observó a lo largo de la traza principal del SFMF.

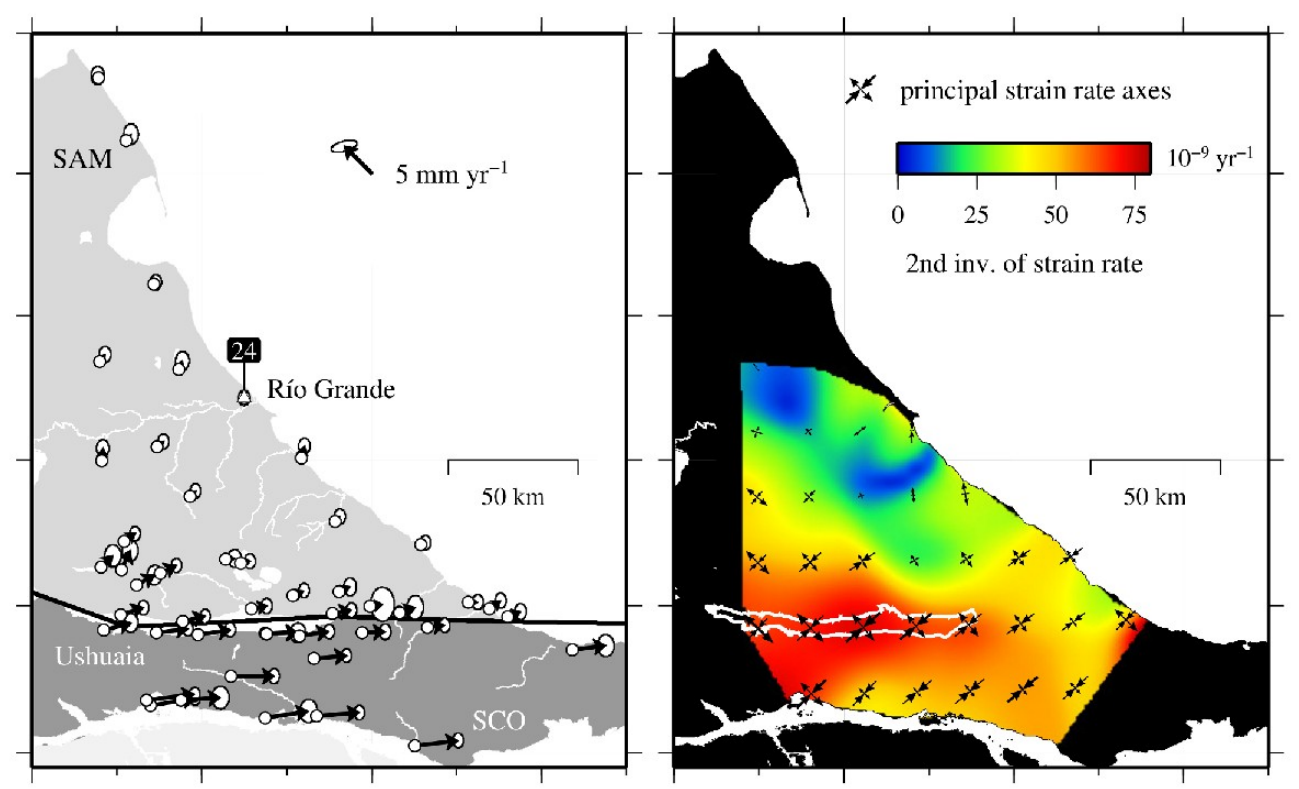

Figura 1.13: Izq.: velocidades intersísmicas observadas referidas a la estación Río Grande (RIOG). Der.: deformación superficial obtenida a partir de invertir las velocidades observadas. La mayor deformación se observa en los alrededores de la falla principal del SFMF (imagen extraída de Mendoza et al., 2015).

En cuanto a la estructura cortical de la región, son muy pocos los estudios que se han realizado con el fin de obtener dicha información en regiones cercanas a TdF. Si bien en esta Tesis se presentan los primeros modelos de velocidades sísmicas y espesores corticales en la zona de estudio, los trabajos previos realizados en regiones cercanas sirven para contrastar los resultados obtenidos en este trabajo. Ludwig et al (1965) presentó el resultado de 22 perfiles de refracción sísmica obtenidos en la zona del Estrecho de Magallanes (situado al oeste de TdF, ver Figura 1.1), obteniendo un espesor cortical de 29.6 a 34.8 km. Por otro lado, a partir de la inversión de formas de ondas regionales, se estimó que el espesor cortical en el extremo sur de Sudamerica varía entre 26 y 36 km (Robertson Maurice et al., 2003). Lawrence y Wiens (2004) realizaron un análisis de funciones del receptor y dispersión de ondas superficiales en la Patagonia Chilena, sugiriendo una profundidad de la Moho con valores comprendidos entre 28 a 34 km. En la presente Tesis se espera estimar valores de espesor cortical, relación de velocidades sísmicas y modelos de velocidad de onda S, con el fin de caracterizar la estructura en el sector oriental de TdF y contrastarlos con los resultados de trabajos previos realizados en zonas cercanas al área de estudio. 


\section{Funciones del Receptor: Teoría y Aplicación}

\subsection{Introducción}

El método de Funciones del Receptor (FRs) comenzó a tener popularidad en el año 1964 cuando Phinney analizó ondas P telesísmicas de largo período con el fin de estimar la estructura de velocidades en la litosfera (Phinney, 1964). Algunos años después, Burdick y Langston (1977) percibieron cierta energía cercana al primer arribo de la onda $\mathrm{P}$ en la componente SV de un sismograma. Modelando las formas de onda correspondientes a diferentes estructuras de velocidad, concluyeron que esa energía era el resultado de una conversión originada en alguna discontinuidad cercana al receptor.

Posteriormente, numerosas investigaciones han analizado la estructura de la corteza utilizando múltiples registros sísmicos pero sólo unas pocas teorías se han desarrollado para estudiar las velocidades de onda S en la corteza y manto superior debajo de una estación de tres componentes. Algunos autores que desarrollaron la técnica fueron Vinnik (1977), Burdick y Langston (1977), Langston (1979, 1981), Owens et al. (1984), Kind y Vinnik (1988), Ammon y Zandt (1993), Cassidy (1995), entre otros.

Cuando se registra una onda sísmica en una estación sismológica, ésta contiene información de la fuente del sismo, de la estructura por la cual se propagó la onda, de la respuesta del instrumento que la registra y de la estructura local cercana al receptor. El método de las FRs consiste en eliminar todos los efectos no deseados en el registro sísmico y mantener sólo aquellos correspondientes a la estructura bajo la estación sismológica. Esta técnica es una de las más utilizadas para estimar la profundidad de la Moho y la relación de velocidades sísmicas en la corteza.

Una FR se puede definir como una serie temporal que, cuando se convoluciona con la componente vertical de un sismograma, reproduce la componente horizontal del mismo, siendo las amplitudes y tiempos de arribo de las fases sensibles a la estructura cercana al receptor (Langston, 1979). 
Esta metodología ha sido desarrollada en el dominio de la frecuencia y en el dominio del tiempo. En el primer caso, Phinney (1964) utilizó la razón del espectro de amplitud para estimar las características de la estructura. Langston (1979) extendió el método utilizando una deconvolución en el dominio frecuencial y su transformada inversa para obtener la respuesta local en el dominio del tiempo. Esta última técnica es una de las más utilizadas para calcular las FRs y es a veces conocida como “water-level deconvolution” (Clayton y Wiggins, 1976; Bostock, 1996; Dueker y Sheehan, 1997; Zhu y Kanamori, 2000; Lawrence y Shearer 2006). Otra aproximación consiste en la deconvolución en el dominio del tiempo (Oldenburg, 1981; Abers et al., 1995; Gurrola et al., 1995; Yuan et al., 1997; Zhu, 2004) que puede ser resuelta utilizando el método de mínimos cuadrados. En 1991, Ammon desarrolló un método de inversión que permitía encontrar contrastes de velocidad sin información previa de la estructura. Este método aplicaba la técnica de deconvolución espectral con water level desarrollado por Clayton y Wiggins, (1976). Algunos años más tarde, Ligorria y Ammon (1999) desarrollaron la "deconvolución iterativa en el dominio del tiempo" siendo una de las más utilizadas hasta el momento por proveer buenos resultados cuando los registros tienen gran contenido de ruido.

Varios autores han utilizado la técnica de FRs para estudiar distribuciones de velocidad de onda $\mathrm{S}$ en corteza y manto superior, para estimar la relación de velocidades sísmicas y espesores corticales (Ammon et al., 1990; Ammon y Zandt, 1993; Zhu y Kanamori, 2000; Tomfohrde y Nowack, 2000; Assumpçao et al., 2002; Gilbert et al., 2006; Perarnau et al., 2010; Gans et al., 2011; Ammirati et al., 2015; Ryan et al., 2016; Akpan et al., 2016; Stanciu et al., 2016), para estudiar la geometría de reflectores (Langston, 1979; Langston, 1981; Cassidy et al., 1998) así como también se ha utilizado la inversión de FRs para determinar la estructura debajo de una estación (Owens et al., 1984; Ammon y Zandt, 1993; Mangino et al.,1993, Perarnau et al., 2010, Ammirati et al., 2013).

La deconvolución en el dominio frecuencial es más eficiente mientras que la deconvolución en el dominio temporal es más estable. Ambas técnicas funcionan bien si la relación señal/ruido (SNR) es alta. En el presente estudio se aplicó la deconvolución iterativa en el dominio del tiempo con el fin de calcular las FRs en TdF.

\section{2 ¿Qué es una Función del Receptor?}

El concepto básico del método es que parte de la energía de una onda P telesísmica incidente en una discontinuidad entre la corteza y manto superior se convertirá en S (Ps) en dicha discontinuidad, y llegará a la estación unos segundos después del arribo de la onda P directa. El arribo inicial en una FR corresponde a la onda P directa. Luego le sigue el arribo de la fase 
convertida Ps que está relacionada con el contraste de impedancia de la Moho, y finalmente se encuentran los arribos de otras fases PpPs y PpSs+PsPs, que son las reverberaciones entre la superficie y los contrastes de impedancia y son conocidas como fases múltiples (Figura 2.1). La amplitud, el tiempo de arribo y la polaridad de las fases convertidas dependerá de la estructura de velocidades de onda S por debajo de la estación.

Las fases convertidas Ps se observan mejor a distancias epicentrales entre $30^{\circ}$ y $90^{\circ}$. Considerar este rango de distancias epicentrales es importante para evitar los efectos de triplicación debido a los gradientes de velocidad en el manto superior (para distancia epicentral $<30^{\circ}$ ) y los efectos de difracción que pueden presentar las fases debido a la interacción con el núcleo (Paulssen y Stutzmann, 1996) (para distancia epicentral $>90^{\circ}$ ).



Figura 2.1: Izquierda: Diagrama de rayos correspondiente a un telesismo arribando a una estación sísmica de tres componentes. Sólo se muestra la onda P directa y las ondas que se convierten en onda S. El resto de las ondas son removidas cuando se genera la FR. Derecha: Se muestra la traza en función del tiempo correspondiente a la FR obtenida a partir del diagrama de la izquierda. Se muestran las principales ondas $\mathrm{P}$ convertidas en un medio sobre un semiespacio. Las letras en minúscula se refieren a las ondas convertidas que se propagan entre la interfase y la superficie libre. (Extraído y modificado de Ammon, 1991).

Las FRs pueden ser calculadas mediante el proceso matemático de deconvolución y como ya se mencionó, este cálculo puede realizarse en el dominio del tiempo o en el de la frecuencia. Es decir, mediante la deconvolución de la componente radial de un sismograma con la componente vertical, se obtiene una forma de onda con amplitudes máximas positivas y negativas que se conoce como “Función del Receptor”. Esos máximos y mínimos corresponden a las conversiones y reverberaciones en la estructura cercana al receptor. 
Si se considera una onda directa P que arriba a una estación sismológica luego de la ocurrencia de un telesismo (Figura 2.2), durante su propagación por el medio, esta onda presentará ciertas reflexiones y refracciones así como también conversiones.


Figura 2.2: a) Ejemplo de un telesismo registrado por la estación TRVA (ver ubicación en la Figura 1.2) donde se observa con claridad el arribo de las ondas de cuerpo P y S y el tren de ondas superficiales (R). Se observan además una ampliación del arribo de la onda P en la componente vertical, norte-sur y este-oeste del 
registro telesísmico y las FR radial y transversal, calculadas a partir de las 3 componentes.

Supongamos que la onda durante su propagación incide con cierto ángulo sobre una discontinuidad, como resultado se tiene una onda P y una onda SV emergentes que llegarán a la estación luego del arribo de la onda P directa. La cantidad de energía de la onda P que se convierte en onda SV dependerá del ángulo de incidencia de la onda inicial P. Cuanto más cercana sea la incidencia a la perpendicular de la discontinuidad, menor será el ángulo. Considerando un modelo de velocidades sísmicas 1D y un ángulo de incidencia agudo (como ocurre para los primeros arribos de telesismos), es posible asumir que toda la energía convertida de la onda P estará en la componente horizontal del receptor.

Además de los arribos directos de las fases convertidas, existen otros rayos que se reflejan una o más veces entre la superficie y una discontinuidad y se denominan fases múltiples. Las cinco reverberaciones con energía significativa generadas por una discontinuidad simple que se han identificado en una FR son: Ps, PpPs, PsPs, PpSs y PsSs (Ammon, 1991). La última reverberación es muy pequeña en comparación a las otras y no es muy utilizada. PsPs y PpSs tienen el mismo tiempo de arribo por contener la misma cantidad de trayectos de ondas P y S, por eso suele denotarse PsPs+PpSs. Para denotar las reverberaciones se utiliza la onda P directa desde la fuente a la Moho, o a una discontinuidad, y cada letra adicional corresponde a un trayecto a través de la corteza de onda P o S, en minúscula se refiere a las ondas que viajan desde la interfase hacia la superficie.

Las FRs se pueden calcular considerando las ondas P, aunque también es posible calcularlas a partir de registros de ondas S como lo han hecho algunos autores (Oreshin et al., 2002; Vinnik et al., 2004a; Angus et al., 2006; Heit et al., 2008; Hansen et al., 2009; Hansen y Dueker, 2009). En el presente trabajo se calcularon las FRs a partir de ondas P telesísmicas. Aunque se utilicen registros de ondas $\mathrm{P}$ durante el procesamiento, las FRs son principalmente sensibles a las velocidades de onda S.

\subsection{Descripción matemática}

Considerando el diagrama de rayos de la Figura 2.1 y asumiendo una estructura de velocidades unidimensional, la respuesta de la Tierra debido a una onda P incidente en dicha discontinuidad puede ser escrita mediante dos componentes de movimiento. La componente vertical Z(t) y la componente radial $\mathrm{R}(\mathrm{t})$ se definen como: 


$$
\begin{aligned}
& Z(t)=\sum_{k=0}^{n} z_{k} s\left(t-t_{k}\right) \\
& R(t)=\sum_{k=0}^{n} r_{k} s\left(t-t_{k}\right)
\end{aligned}
$$

siendo $\mathrm{s}(\mathrm{t})$ la señal temporal de la fuente, $\mathrm{z}_{\mathrm{k}} \mathrm{y} \mathrm{r}_{\mathrm{k}}$ las amplitudes del k-ésimo rayo en cada componente $\mathrm{y}_{\mathrm{k}}$ el tiempo de arribo en la superficie. La suma se considera para $\mathrm{n}$ rayos obteniendo la onda directa $\mathrm{P}$ cuando $\mathrm{k}=0$.

Asumiendo que $s\left(t-t_{k}\right)$ es una función delta, las transformadas de Fourier de ambas componentes se pueden escribir como:

$$
\begin{aligned}
& Z(w)=z_{0} \sum_{k=0}^{n} \hat{z_{k}} \mathrm{e}^{-i w t_{k}} \\
& R(w)=r_{0} \sum_{k=0}^{n} \hat{r_{k}} \mathrm{e}^{-i w t_{k}}
\end{aligned}
$$

siendo $\hat{z_{k}}=z_{k} / z_{0}$ la amplitud del k-ésimo rayo normalizada por la amplitud del rayo $\mathrm{z}_{0}$ que corresponde al arribo directo de la onda P y $w$ la frecuencia radial. Lo mismo será para el caso de $\hat{r_{k}}$. En la Figura 2.3 se indican $\mathrm{z}_{0} \mathrm{y} \mathrm{r}_{0}$.

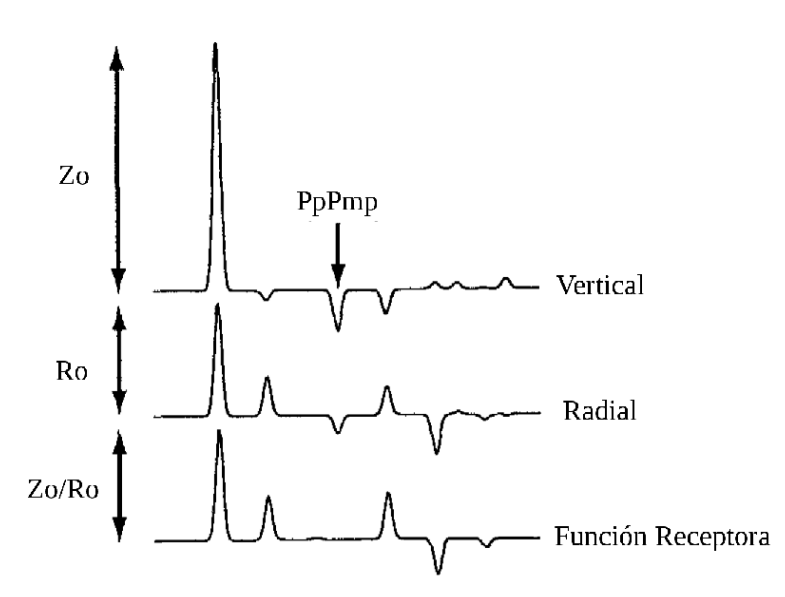

Figura 2.3: Esquema de la respuesta vertical y radial debido a una señal telesísmica que se registra en una estación de tres componentes y su FR resultante. Notar que la fase PpPmp (o PpPp) que indica la reverberación de la onda $\mathrm{P}$ entre la Moho y la superficie, no está presente en la FR ya que arriba con el mismo ángulo de incidencia que la onda directa P y es removida mediante el proceso de equalización de la fuente. (Extraído y modificado de Ammon, 1991). 
El procedimiento de ecualización de la fuente propuesto por Langston (1979) consiste en realizar la deconvolución de la componente vertical con la componente radial del movimiento:

$$
H(w)=\frac{S(w) R(w)}{S(w) Z(w)}=\frac{R(w)}{Z(w)}
$$

siendo $\mathrm{S}(\mathrm{w})$ el espectro de la fuente y $\mathrm{H}(\mathrm{w})$ la transformada de Fourier de la FR radial $\mathrm{h}(\mathrm{t})$. La ecuación (2.5) es lo que normalmente se utiliza para definir la FR en el dominio de la frecuencia. De la misma manera es posible obtener la FR transversal, dividiendo las componentes transversal y vertical en el dominio de la frecuencia.

Es necesario asumir que $\mathrm{S}(\mathrm{w})$ y $\mathrm{Z}(\mathrm{w})$ son distintas de cero lo cual puede no ser el caso cuando se trabaja con datos reales. Este problema se puede resolver mediante el método de "water-level deconvolution" (Clayton y Wiggins, 1976), en el cual los valores pequeños del denominador de la ecuación (2.5) son reemplazados por una fracción del máximo valor del denominador (Figura 2.4). Con este método se evita una división por cero o por valores pequeños y se reduce el efecto de elementos de baja frecuencia en las FRs haciendo que el proceso sea numéricamente estable.

De esta manera la FR se puede escribir como:

$$
H(w)=\frac{R(w) \ddot{Z}(w)}{Z(w) \ddot{Z}(w)}
$$

donde " denota al complejo conjugado, siendo $\frac{1}{Z}=\frac{\ddot{Z}}{Z \ddot{Z}}$

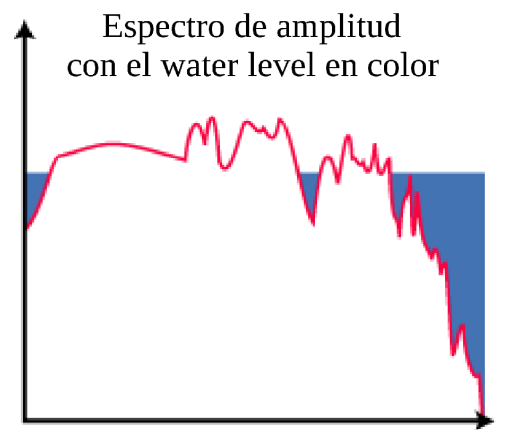

Frecuencia



Frecuencia

Figura 2.4: Método de deconvolución de water-level (Extraído y modificado de Ammon, 2006).

El método de water-level reemplaza la ecuación (2.6) por la siguiente expresión:

$$
H(w)=\frac{R(w) \ddot{Z}(w)}{f(w)} G(w)
$$


donde

$$
f(w)=\max [Z(w) \ddot{Z}(w), c \max \{Z(w) \ddot{Z}(w)\}]
$$

$\mathrm{y}$

$$
G(w)=N \exp \left(\frac{-w^{2}}{4 \mathrm{a}^{2}}\right)
$$

En la ecuación (2.8), “c” determina el mínimo valor permitido en el denominador (denotado como water level), "N” es la constante de normalización, $w$ la frecuencia y G(w) el filtro Gaussiano con pulsos de ancho "a”.

\subsubsection{Filtro Gaussiano}

El filtro Gaussiano G de la ecuación (2.9) tiene un solo parámetro que controla la respuesta en frecuencia y es el valor de “a”. La selección de este parámetro afectará por lo tanto al cálculo de las FRs en cuanto al contenido frecuencial. El parámetro "a” controla el ancho del pulso Gaussiano y el contenido frecuencial de la FR.

Algunos autores han utilizado un filtro Gaussiano con valor de 5.0 (Zhu y Kanamori, 2000), mientras que otros han utilizado un valor de 2.0 (Gilbert y Sheehan, 2004). En la Tabla 2.1 se observan algunos valores del parámetro "a” y la correspondiente frecuencia cuando $G(w)=0.1$.

\begin{tabular}{cc}
\hline Valor de a & $\begin{array}{c}\text { Frecuencia (Hz) cuando } \\
\mathrm{G}(\mathrm{w})=0.1\end{array}$ \\
\hline 5 & 2.4 \\
2.5 & 1.2 \\
2 & 1.0 \\
1.25 & 0.6 \\
1 & 0.5 \\
\hline
\end{tabular}

Tabla 2.1: Valores de "a" correspondiente al valor de frecuencia cuando $G(w)=0.1$

En general, valores pequeños de “a” (por ejemplo 1 o 2) se utilizan para examinar el contenido de baja frecuencia presente en los datos, mientras que valores grandes (por ejemplo 5) se utilizan para analizar contenido de alta frecuencia (Cassidy, 1992). Las FRs calculadas con valores grandes de "a”, permiten analizar con más detalle las estructuras, mientras que las calculadas con valores pequeños, ofrecen información más general y simple. En la Figura 2.5, se compara una FR 
calculada con un valor de a=2.5 (baja frecuencia) con otra FR calculada con un valor de $a=5$ (frecuencias más altas). En ambos casos una conversión Ps se observa entre 4 y 5 s, pero en la FR calculada con un valor mayor de "a” se observan además la presencia de otras fases asociadas a la estructura superficial (con tiempos de arribo $\sim 1$ s y 2.7 s).

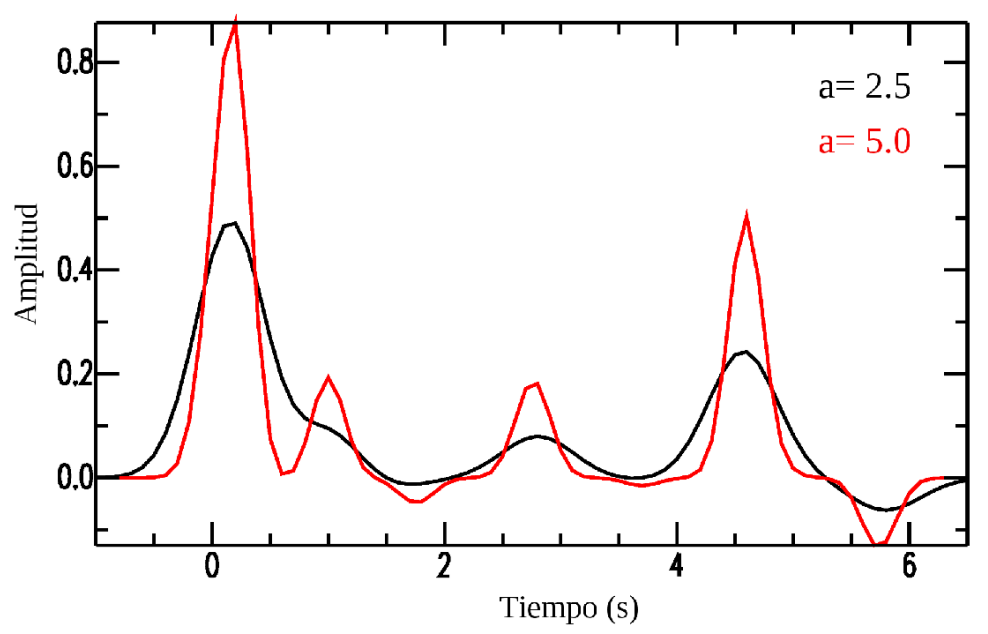

Figura 2.5: FRs radiales calculadas con un valor del factor “a” igual a 2.5 (en negro) y 5.0 (en rojo) a partir de un telesismo registrado en la estación TRVA (ver ubicación en la Figura 1.2). Los datos del telesismo son: Fecha: 22/02/2006, Hora origen: 22:19:07.8, Distancia epicentral: 78.9º Profundidad: 11 km y Magnitud: 7.

\subsubsection{Ejemplo con tres arribos}

Si se considera los primeros tres claros arribos que llegan a una estación: la onda P directa, la onda P reflejada en la interfase por ejemplo corteza-manto superior y la onda P convertida en S en dicha interfase (ver Figura 2.1). Así, una onda P incidente en una discontinuidad que separa dos medios con diferentes velocidades sísmicas, produce una onda reflejada y refractada a lo largo de la interfase. Si el medio no es fluido se generará una onda S. En el caso de ondas telesísmicas, la onda convertida a S posee más energía en la componente horizontal que en la vertical del sismograma.

La transformada de Fourier del movimiento de la componente radial $\mathrm{R}$ y vertical $\mathrm{Z}$ de los tres arribos se puede escribir como sigue:

$$
\begin{aligned}
& R(w)=r_{0}\left(1+\hat{r_{p}} e^{-i w t_{p}}+\hat{r}_{s} e^{-i w t_{s}}\right) \\
& Z(w)=z_{0}\left(1+\hat{z_{p}} e^{-i w t_{p}}+\hat{z_{s}} e^{-i w t_{s}}\right)
\end{aligned}
$$


El primer término dentro del paréntesis de la derecha en las dos ecuaciones anteriores corresponde a la onda P directa, el segundo término corresponde a la onda P reflejada y el tercero a la onda S. La mayor parte de energía de la onda S se encuentra en la componente horizontal, por lo que es claro que la amplitud de la onda S relativa a la amplitud de la onda directa P debe ser mucho menor que 1. Si se toma en cuenta esto junto con las ecuaciones (2.5), (2.10) y (2.11) se obtiene:

$$
H(w)=\frac{r_{0}}{z_{0}} \frac{1+\hat{r_{p}} e^{-i w t_{p}}+\hat{r_{s}} e^{-i w t_{s}}}{1+\hat{z_{p}} e^{-i w t_{p}}}
$$

Para una onda plana que llega a una interfase horizontal los rayos son paralelos entre sí y $\hat{r_{p}}=\hat{z_{p}}$ entonces se puede escribir:

$$
H(w)=\frac{r_{0}}{z_{0}} \frac{1+\hat{z_{p}} e^{-i w t_{p}}+\hat{r_{s}} e^{-i w t_{s}}}{1+\hat{z_{p}} e^{-i w t_{p}}}
$$

$\hat{z_{p}}$ es la amplitud relativa de la onda reflejada $\mathrm{P}$ y generalmente es muy pequeña respecto a la amplitud de la onda directa P. Si se aplica un desarrollo de Taylor en el denominador de la ecuación (2.13):

$$
\frac{1}{1+\hat{z_{p}} e^{-i \omega t_{p}}}=1-\left(\hat{z}_{p} e^{-i w t_{p}}\right)+\left(\hat{z}_{p} e^{-i w t_{p}}\right)^{2}-\left(\hat{z_{p}} e^{-i w t_{p}}\right)^{3}+\ldots
$$

y considerando sólo los términos de orden menor que 1 del desarrollo se puede escribir:

$$
H(w)=\frac{r_{0}}{z_{0}}\left(1+\hat{r}_{s} e^{-i w t_{s}}+\hat{z}_{p} e^{-i w t_{p}}\right)\left(1-\hat{z}_{p} e^{-i w t_{p}}\right)
$$

Despreciando los términos de orden menor que cero en la multiplicación anterior se obtiene:

$$
H(w)=\frac{r_{0}}{z_{0}}\left(1+\hat{r}_{s} e^{-i w t_{s}}\right)
$$

y en el dominio del tiempo:

$$
h(t)=\frac{r_{0}}{z_{0}}\left(\delta(t)+\hat{r}_{s} \delta\left(t-t_{k}\right)\right)
$$

siendo $\quad \delta(t)$ la función delta. 
Se puede concluir a partir de la última ecuación que sólo la onda directa $\mathrm{P}$ y la convertida a $\mathrm{S}$ estarán presentes en la FR. Este procedimiento se puede realizar de la misma manera considerando múltiples capas (Ammon, 1991).

\subsubsection{Deconvolución iterativa en el dominio del tiempo}

Ligorria y Ammon (1999), propusieron la técnica de la deconvolución iterativa en el dominio del tiempo la cual asume que una FR consiste en una superposición de impulsos múltiples. El concepto matemático de esta técnica está desarrollado en Kikuchi y Kanamori (1982). El fundamento se basa en una minimización por el método de mínimos cuadrados de la diferencia entre la componente horizontal del sismograma y una señal calculada mediante la convolución de un tren iterativo de pulsos con la componente vertical del sismograma. Esta técnica se puede aplicar tanto para la componente radial como la transversal. Ya que los impulsos implican un rango de frecuencias infinitas, se utiliza un ancho de banda delimitado y en la mayoría de casos los pulsos son Gaussianos, es decir pulsos cuya envolvente es una función Gaussiana. De esta manera, realizando la correlación cruzada de las dos componentes es posible determinar el tiempo del primer pulso en la función correlacionada. Este resultado se convoluciona con la componente vertical del sismograma y es sustraído de la componente radial original. Luego se realiza nuevamente la correlación cruzada del resultado anterior con la componente vertical. Este proceso se repite varias veces. Cuando se dispone de datos de banda ancha con buena SNR, cualquiera de los métodos de deconvolución funcionan bien. Pero en presencia de datos con mala SNR, el método de deconvolución iterativa en el dominio del tiempo es uno de los más utilizados por no presentar inestabilidades y aunque es un poco más lento en su cómputo, suele ser mejor en procesos de automatización.

\subsection{Aplicación de la técnica en TdF}

En el siguiente diagrama se muestran los pasos llevados a cabo en el procedimiento para el cálculo de las FRs en TdF y que serán detallados a continuación (Figura 2.6): 




Figura 2.6: Diagrama de los pasos realizados con el fin de calcular las FRs en TdF y ejemplo de una FR radial obtenida en las estaciones sismológicas TRVA, DSPA, USHA y ELCA respectivamente (la ubicación de las estaciones se muestra en la Figura 1.2 del Capítulo I).

\subsubsection{Selección de datos}

Los datos utilizados han sido registrados en cuatro estaciones sismológicas instaladas en la parte argentina de TdF: DSPA, ELCA, TRVA y USHA. La ubicación de las estaciones está marcada con estrellas en el mapa de la Figura 1.2 del Capítulo I. Todas las estaciones son de banda ancha como se mencionó en la sección 1.6 del Capítulo I, localizadas en sitios remotos y funcionando con energía eléctrica y solar.

La selección de eventos telesísmicos se realizó considerando un catálogo de sismicidad obtenido por el National Earthquake Information Center (NEIC-USGS) para el período enero 2006- diciembre 2010. El catálogo está compuesto por telesismos con magnitud $\mathrm{m}_{\mathrm{b}}>5.5 \mathrm{y}$ distancia epicentral entre $30^{\circ}$ y $90^{\circ}$. El rango de distancia epicentral fue seleccionado con el fin de evitar fases triplicadas provenientes de las zonas de transición del manto inferior y superior como se mencionó en la sección 2.2.

Se analizó uno por uno cada registro de cada estación con el fin de seleccionar los telesismos que tuvieran un claro arribo de la onda P directa con buena SNR. Dependiendo la disponibilidad de los datos y la calidad en cada estación, se reunieron un total de 309 telesismos (Figura 2.7 y Tabla A.1 del Apéndice A). 




Figura 2.7: Ubicación epicentral de los telesismos analizados (en azul y rojo) y los seleccionados posteriormente con buena calidad de FR (en azul). Los tres círculos concéntricos determinan la distancia epicentral de $30^{\circ}, 60^{\circ}$ y $90^{\circ}$. TdF está indicado con una estrella en el centro del mapa generado mediante el software GMT (Wessel y Smith, 1995).

\subsubsection{Preprocesamiento de los datos}

Se utilizó el software Seismic Analysis Code (Goldstein y Snoke, 2005) para preprocesar los sismogramas. Los pasos del preprocesado fueron los siguientes:

\subsubsection{Conversión del formato y preparación del header}

Los registros de las cuatro estaciones fueron convertidos a formato SAC. Posteriormente, fueron cortados considerando una ventada de 80 segundos de tiempo que comenzaba 5 segundos antes del arribo directo de la onda P. Luego se incorporó la información de ubicación y hora origen de cada telesismo en los respectivos SAC headers de cada registro. El header o encabezamiento de cada registro contiene información acerca del dato registrado. Los datos incorporados fueron la latitud, longitud, profundidad del evento y la hora origen. Esta información es necesaria para el cálculo de las FRs. 


\subsubsection{Reducción de la media y de la pendiente}

El procedimiento para remover la media de un registro consiste en desplazar la línea media de las amplitudes del mismo a nivel cero. Por otro lado, se eliminó la pendiente con el fin de eliminar de los datos cualquier sesgo y poder obtener una media horizontal (Figura 2.8).



b)

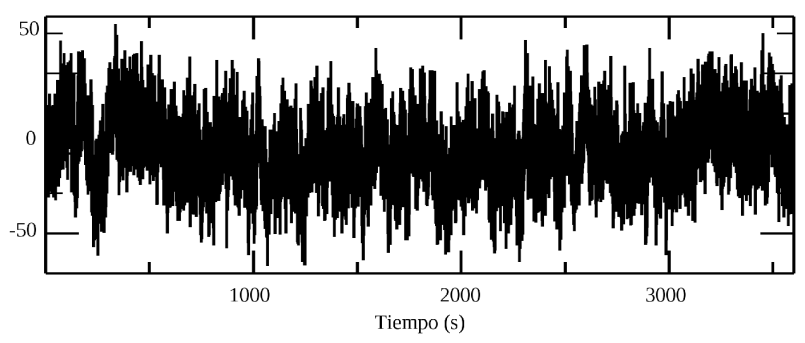

Figura 2.8: a) Registro obtenido en la estación TRVA sin preprocesar (componente norte-sur). b) Mismo registro que (a) pero luego de remover la media y la pendiente.

Estos dos pasos son necesarios en el tratamiento de señales sísmicas y se aplican con los comandos "rmean" y "rtrend" dentro del software SAC.

\subsubsection{Rotación de las componentes}

El movimiento generado por un terremoto es registrado por un sismómetro a lo largo de las direcciones Vertical (Z), Norte-Sur (N-S) y Este-Oeste (E-O). Sin embargo los datos registrados no están alineados con los ejes en los cuales se propaga la energía liberada siendo necesario una rotación de las componentes que lo ha registrado. Existen dos tipos de rotación: el sistema de rotación ZRT, que es una rotación bidimensional, y el sistema de rotación LQT, siendo este último una rotación tridimensional. En el sistema ZRT, la componente vertical tiene la misma dirección que la original registrada por el dato (Figura 2.9). Este sistema entonces rota las componentes 
horizontales N-S y E-O a componentes radial y transversal R y T de la siguiente manera:

$$
\left(\begin{array}{l}
R \\
T \\
Z
\end{array}\right)=\left(\begin{array}{ccc}
\cos (\theta) & \sin (\theta) & 0 \\
-\sin (\theta) & \cos (\theta) & 0 \\
0 & 0 & 1
\end{array}\right)\left(\begin{array}{c}
E \\
N \\
Z
\end{array}\right)
$$

siendo $\quad \theta=\frac{3 \Pi}{2}-\Phi \quad$ y $\Phi$ el backazimut (BAZ).

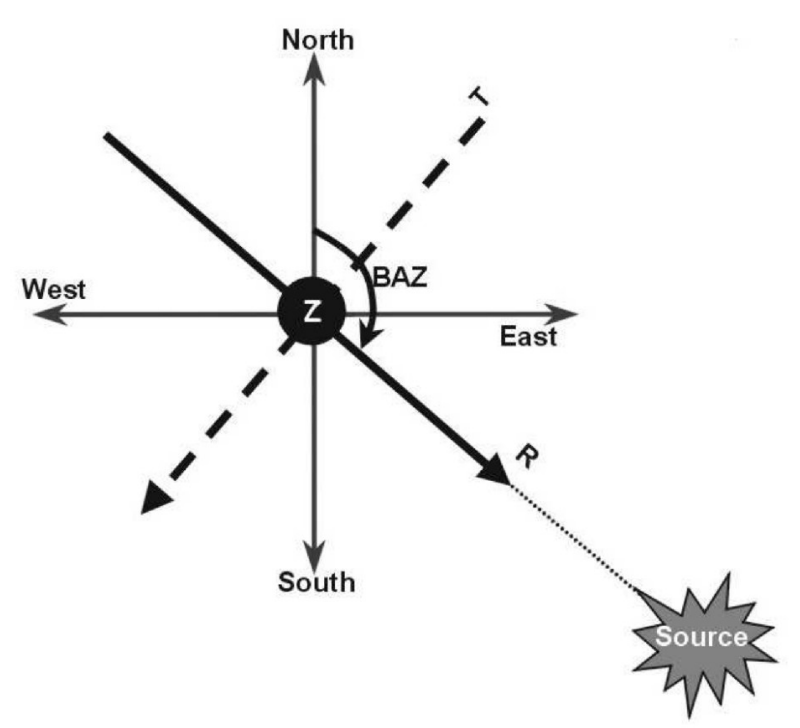

Figura 2.9: Esquema de rotación del sistema ZNE al sistema ZRT. (Extraído de Ekrem, 2002).

En este trabajo se aplicó una rotación bidimensional.

\subsubsection{Filtro pasabanda}

Los datos fueron filtrados con un filtro pasabanda en tres rangos de frecuencia diferentes: 0.02$1 \mathrm{~Hz}, 0.5-2 \mathrm{~Hz}$ y $0.08-2 \mathrm{~Hz}$ (Figura 2.10). Utilizar FRs calculadas con bajas frecuencias puede reducir el potencial de resultados sesgados por energía de scattering que expresa el decaimiento de la amplitud de las ondas cuando se propagan (Mangino et al., 1993). Sin embargo la selección de un rango de frecuencias bajas puede complicar la identificación de discontinuidades superficiales. En el caso de TdF, se realizó una prueba preliminar en diferentes bandas de frecuencias y se seleccionó aquella que daba mejores formas de onda y un claro arribo de la fase Ps. 


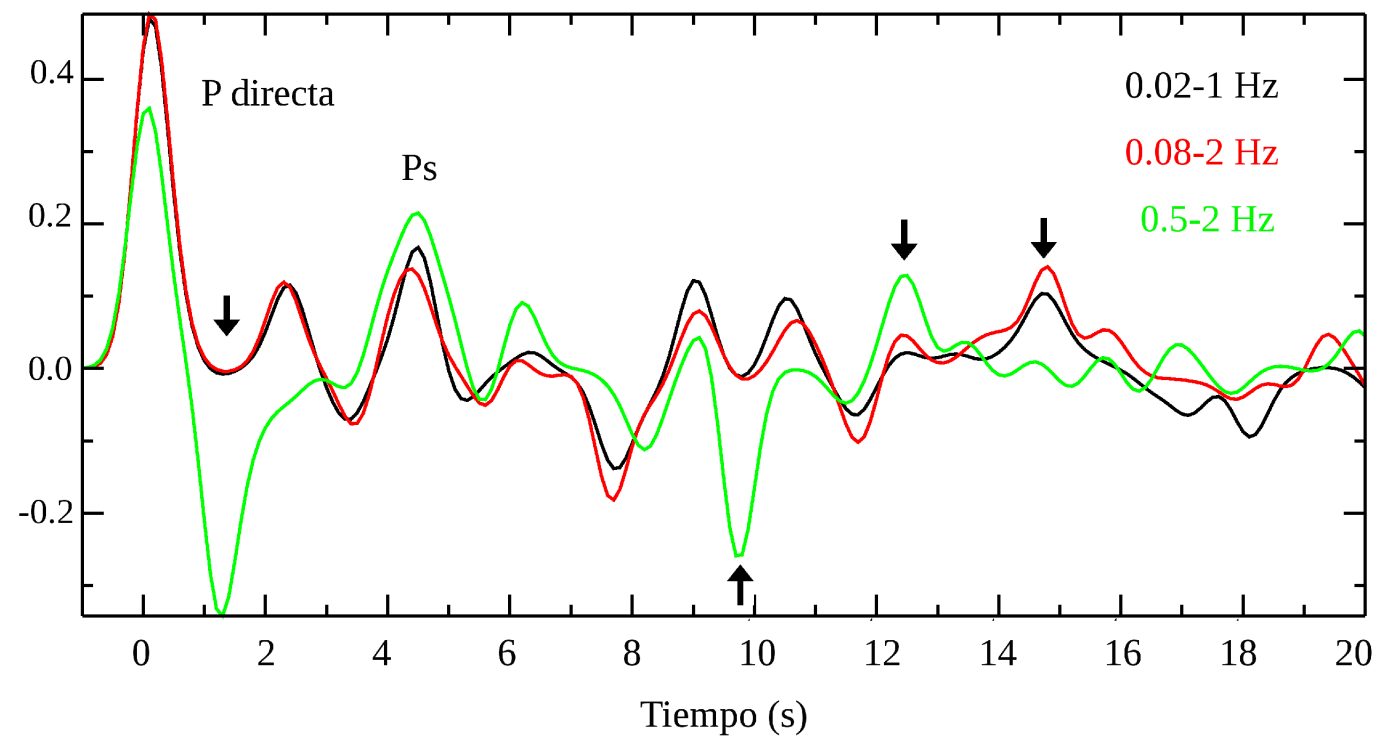

Figura 2.10: Ejemplo del efecto del filtro pasabanda utilizado en el cálculo de las FRs radiales en TdF. Las FRs fueron calculadas a partir del mismo evento telesísmico filtrado en tres rangos de frecuencia diferente: 0.02-1 Hz (negro), 0.08-2 Hz (rojo) y 0.5-2 Hz (verde). Las flechas negras indican las principales diferencias observadas en cuanto a amplitud de las distintas fases que arriban.



Figura 2.11: Ejemplo de una FR radial calculada a partir de un telesismo registrado en la estación TRVA pero con el dato preprocesado en tres rangos de frecuencia diferentes. La línea roja punteada indica el arribo de la fase Ps que se observa claramente cuando los datos son filtrados con un filtro pasabanda 0.02-1 Hz, y no se observa con la misma claridad en los otros dos casos. 
Según una inspección visual, en la mayoría de los resultados no se observó un claro arribo de la fase convertida Ps cuando se utilizaban los rangos de frecuencia $0.5-2 \mathrm{~Hz}$ y $0.08-2 \mathrm{~Hz}$ (Figura 2.11). Para el posterior análisis de las FRs se seleccionaron aquellas calculadas en el rango de frecuencias de $0.02-1 \mathrm{~Hz}$.

\subsubsection{Cálculo de las FRs}

Una vez preprocesados los datos se procedió a la aplicación del algoritmo de deconvolución iterativa en el dominio del tiempo propuesto por Ligorria y Ammon (1999). Antes de calcular la deconvolución se aplicó un filtro Gaussiano con un factor de a=2.5. El significado del parámetro "a” fue discutido en la sección 2.3.1. La selección de este valor depende de cada problema en particular. En el caso de TdF, las FRs fueron calculadas con distintos valores de filtros Gaussianos desde 1.0 a 5.0. Para el análisis posterior de las mismas, se seleccionó aquellas calculadas con un filtro Gaussiano de 2.5 ya que correspondían a las FR obtenidas que presentaron la conversión Ps con mayor claridad en su arribo.

El presente estudio se enfocó en el cálculo de las FRs radiales a partir de registros telesísmicos de onda P. En el caso de USHA, se analizó un caso en particular siendo necesario el cálculo de las FRs transversales (ver sección 2.5.4.1).

\subsection{Resultados}

Con el fin de analizar los resultados en cada estación, se seleccionaron aquellas FRs que cumplieran con el siguiente criterio:

$\rightarrow$ claro arribo de la conversión Ps

$\rightarrow$ primer pulso positivo, correspondiente al arribo de la onda directa P con un tiempo de arribo igual a $0 \mathrm{~s}$

Es importante destacar que, debido a la presencia de ruido en los datos, muchas de las FRs obtenidas no cumplieron con el criterio anterior y desafortunadamente tuvieron que ser descartadas como se mencionó en la sección 2.4.1. Para el posterior análisis se seleccionaron un total de 106 FRs entre las cuatro estaciones (Tabla 2.2). Los telesismos correspondientes a las FRs seleccionadas están indicados en el mapa de la Figura 2.7 de la sección 2.4.1 (en color azul). 
Las FRs obtenidas en todas las estaciones muestran un grado de complejidad que era de esperar debido al ambiente geotéctonico presente en la zona. En el mapa de la Figura 1.2 (Capítulo I, sección 1.3) se observa que todas las estaciones están localizadas cerca de sistemas de fallas y en los márgenes de diferentes unidades estructurales. A pesar de esto, en todas las FRs seleccionadas se observa el arribo de la fase convertida Ps. Las reberveraciones PpPs y PpSs+PsPs fueron más difíciles de identificar debido a la complejidad de los datos.

\begin{tabular}{ccccc}
\hline \hline Estación & Latitud $\left(\mathbf{(}^{\circ}\right)$ & Longitud $\left(^{(}\right)$ & Telesismos & FRs \\
\hline \hline TRVA & -54.68 & -67.34 & 116 & 42 \\
USHA & -54.81 & -68.43 & 52 & 27 \\
DSPA & -53.95 & -68.26 & 101 & 25 \\
ELCA & -54.34 & -67.85 & 40 & 12 \\
\hline \hline
\end{tabular}

Tabla 2.2. Número de telesismos utilizados para el cálculo (indicados en rojo en la Figura 2.7) y número de FRs seleccionadas en cada estación (calculadas a partir de los telesismos indicados en azul en la Figura 2.7).

\subsubsection{Estación TRVA}

En la Figura 2.12 se observan las FRs seleccionadas en la estación TRVA en función del BAZ. Luego de la selección de las mismas en esta estación, así como en las estaciones DSPA y USHA, se observó que los telesismos correspondientes a las FRs están principalmente localizados hacia el SO y N de TdF. Además las FRs seleccionadas demostraron tener patrones similares en ambos grupos ( $\mathrm{N}$ y SO) de BAZ por lo tanto, para el posterior análisis en los siguientes capítulos, se separó a las FRs en dos grupos teniendo en cuenta los puntos de conversión: grupo 1 (eventos provenientes del SO) y grupo 2 (eventos provenientes del $\mathrm{N}$ ).

En esta estación TRVA, el arribo de la fase convertida Ps se observó alrededor de los 4.2 s y $4 \mathrm{~s}$ después del arribo de la onda directa $\mathrm{P}$ para el grupo 1 y para el grupo 2 respectivamente. Las FRs correspondientes al grupo 2 mostraron mayor complejidad que las del grupo 1. Se observó además una significativa fase negativa entre los 6.5 y $8 \mathrm{~s}$ en todas las FRs del grupo 1 y en algunas del grupo 2. A pesar de que el límite litosfera-astenósfera (LAB) es más fácil de detectar a partir de la técnica de FRs de ondas S (Li et al., 2004; Kumar et al., 2005; Rychert et al., 2007; Abt et al., 2010; Ford et al., 2010; Rychert y Harmon 2016), dicha fase negativa podría estar indicando el LAB en la zona de TdF. 
BAZ (⿳)
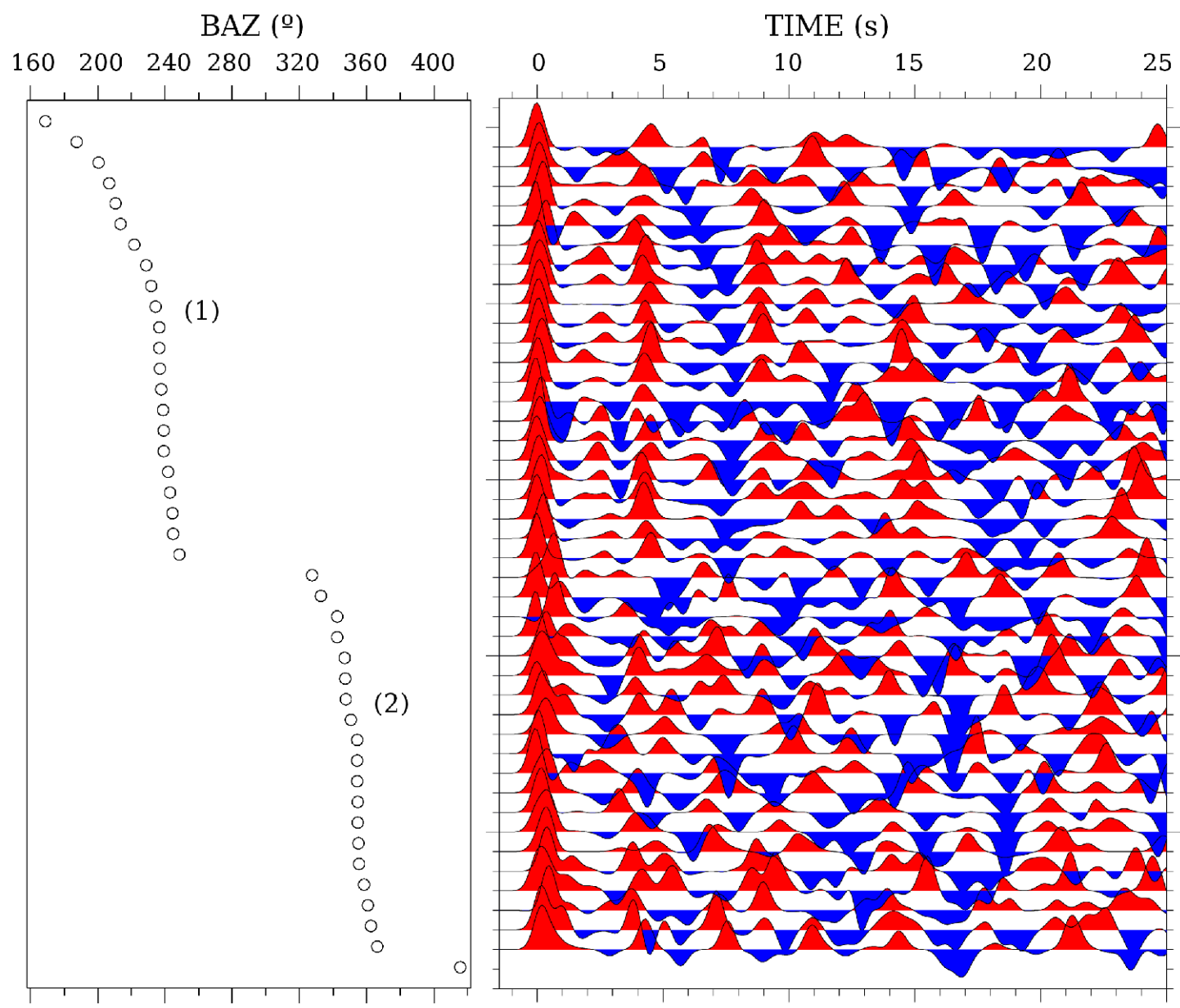

Figura 2.12: FRs radiales seleccionadas en la estación TRVA. (1) y (2) indican los grupos 1 (eventos provenientes del SO) y grupo 2 (eventos provenientes del N), respectivamente. En todas las estaciones las FRs fueron graficadas utilizando el software GSAC (Herrmann, 2013).

\subsubsection{Estación USHA}

A pesar del gran número de FRs descartadas, las FRs de mejor calidad seleccionadas fueron observadas en las estaciones USHA y TRVA. En el caso de la estación USHA, el arribo de la fase convertida Ps se observó entre los 3 y 3.3 s luego del arribo de la onda directa P, y a diferencia de TRVA, arribando antes y con mayor amplitud para el grupo 1 (Figura 2.13). La conversión posiblemente proveniente del LAB también parece observarse en USHA en todos los eventos entre los 7.5 y 8 s. Se observó además un claro cambio de polaridad en función del BAZ para la fase con arribo entre los 1.3 y 1.5 s, siendo negativa para las FRs del grupo 1 y positiva para el grupo 2. Los cambios de polaridad pueden ser causados por la presencia de anisotropía y/o discontinuidades con 
inclinación (Cassidy, 1992; Peng y Humphreys, 1997; Savage, 1998). Este caso en particular es analizado con más detalle en la sección 2.5.4.1. Entre los $10 \mathrm{~s}$ y los $15 \mathrm{~s}$ se observa una fase positiva que podría estar indicando la conversión PpPs.

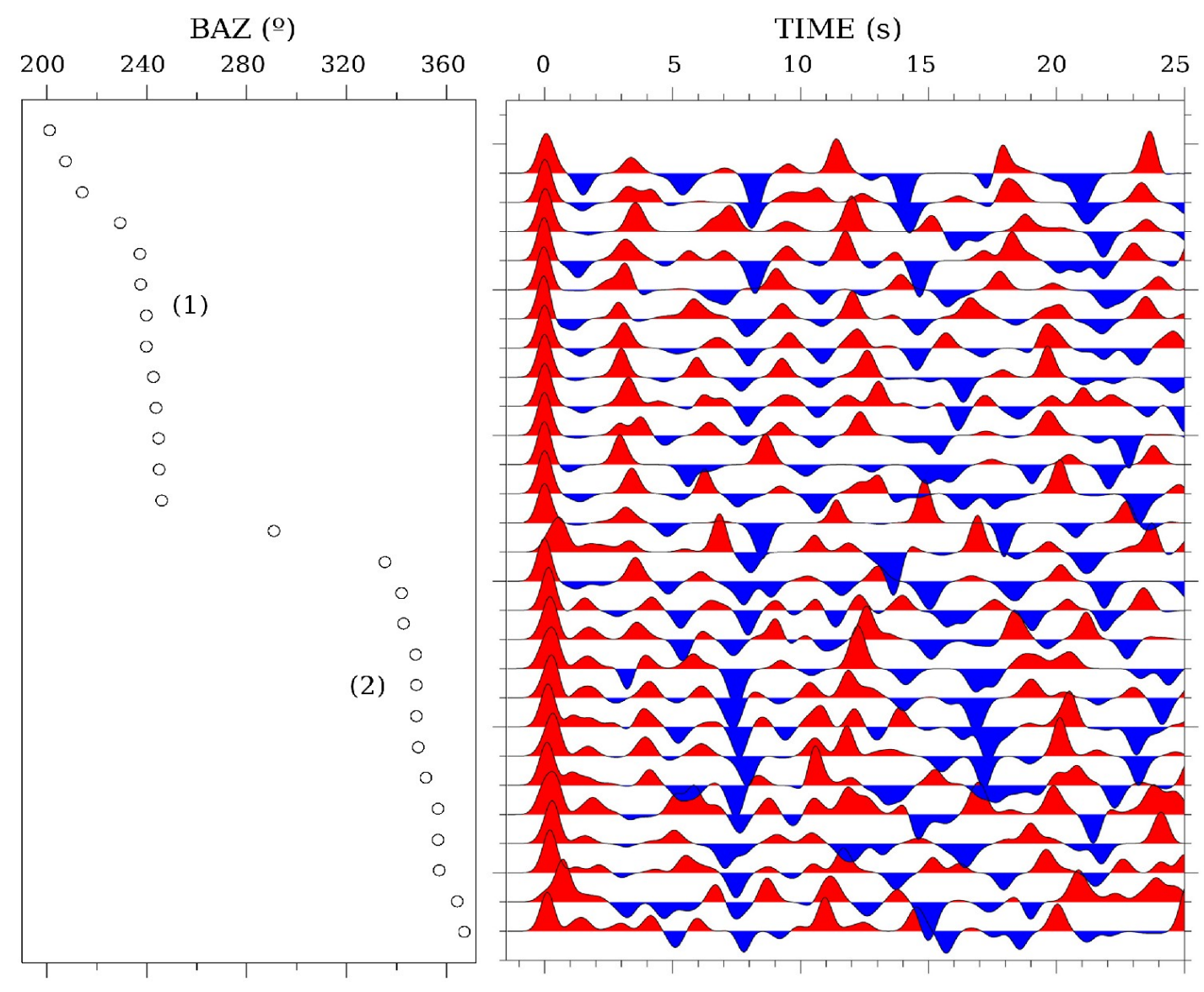

Figura 2.13: FRs radiales seleccionadas en la estación USHA. (1) y (2) indican los grupos 1 (eventos provenientes del SO) y grupo 2 (eventos provenientes del $\mathrm{N}$ ), respectivamente.

\subsubsection{Estación DSPA}

Las FRs obtenidas en la estación DSPA muestran similar a mayor complejidad en comparación con las obtenidas en las estaciones TRVA y USHA (Figura 2.14). A pesar de que DSPA es la estación en la que se tuvieron que descartar el mayor número de FRs, fue posible identificar la conversión Ps en la mayoría de las FRs radiales seleccionadas. Dicho arribo se observó entre los 33.5 s luego del arribo de la onda directa P. Las FRs seleccionadas en este caso también se agruparon según el BAZ en grupo 1 y grupo 2 como en las estaciones TRVA y USHA.. Se observa una considerable fase positiva alrededor de los 9-9.5 s para el grupo 2. Las FRs del grupo 1 muestran 
mayor complejidad en sus formas de onda.

En el grupo 2 se identifica una fase negativa entre los $15-20$ s que podría estar indicando la fase múltiple PpSs+PsPs.
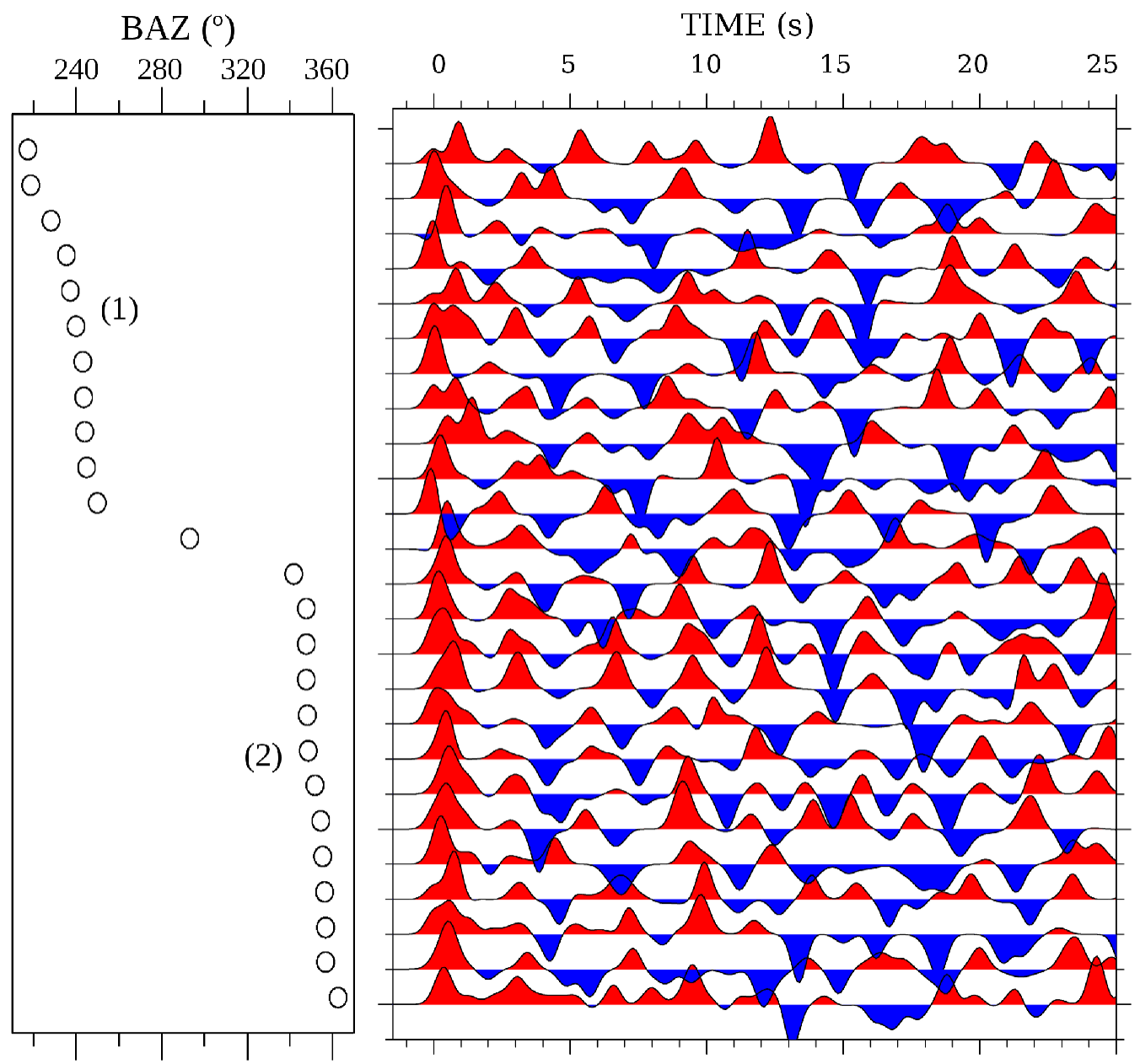

Figura 2.14: FRs radiales seleccionadas en la estación DSPA. (1) y (2) indican los grupos 1 (eventos provenientes del SO) y grupo 2 (eventos provenientes del $\mathrm{N}$ ), respectivamente.

\subsubsection{Estación ELCA}

Los eventos utilizados en el posterior análisis de las FRs se analizaron considerando dos clusters de telesismos ( $\mathrm{SO}$ y $\mathrm{N}$ ) no siendo el caso de ELCA por el escaso número de FRs seleccionadas. En este caso se obtuvieron muy pocas FRs de buena calidad correspondientes a telesismos ubicado al SO de la estación (grupo 1) en su mayoría. Para esta estación ELCA el 
análisis posterior se realizó teniendo en cuenta sólo este grupo de FRs. En la Figura 2.15 se observan las 12 formas de ondas seleccionadas. Las fases múltiples fueron más difíciles de identificar en esta estación.
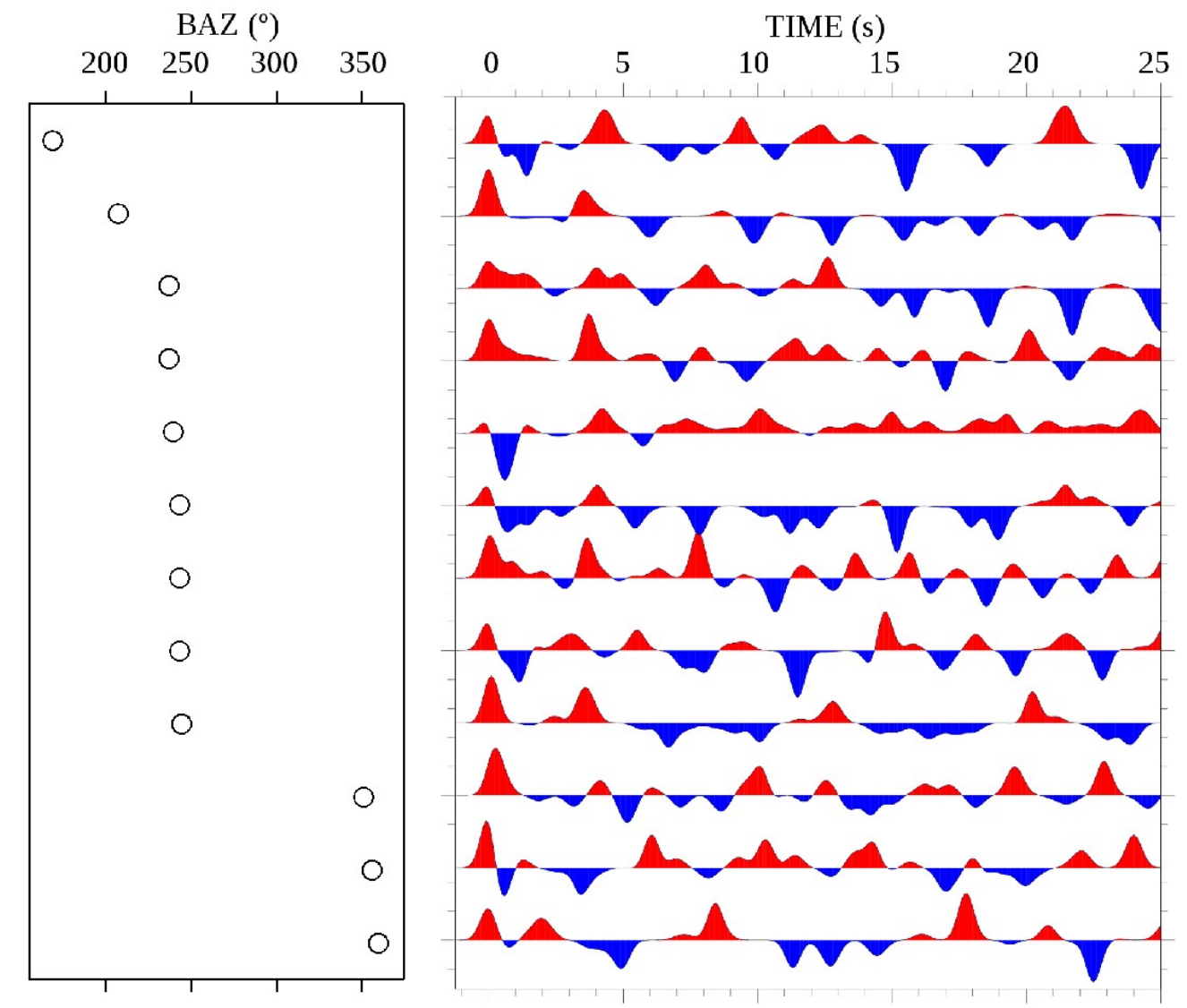

Figura 2.15: FRs radiales seleccionadas en la estación ELCA.

\subsubsection{Heterogeneidades en la estación USHA}

El análisis de FRs se basa en una teoría de capas horizontales. Sin embargo, la presencia de diversidas fallas y pliegues que caracterizan el ambiente geotectónico de TdF genera FRs cuyas formas de onda a menudo no son correctamente modeladas alterando el mejor ajuste de datos y modelo de capas horizontales. Por este motivo, es importante tener presente que para aplicar la técnica de FRs se asumió un modelo de capas horizontales y no se han considerado heterogeneidades. Sin embargo, en el análisis de los resultados obtenidos, se observó la presencia de una fase con un notorio cambio de polaridad en función del BAZ en la estación USHA.

Si se considera un modelo unidimensional e isótropo de capas horizontales, las conversiones Ps 
sólo se observarán en las componentes vertical y radial de un sismograma. Sin embargo, la presencia de ciertas complejidades en la Tierra como capas con anisotropía, inclinación o el scattering de las señales sísmicas, generan ondas con energía en las componentes transversales (Fouch y Rondenay, 2006). Además una interfase con inclinación o anisotropía conduce a variaciones en el tiempo de viaje y en la amplitud de las ondas.

Casiddy (1992), Peng y Humphreys (1997) y Savage (1998) son algunos de los trabajos que han analizado la presencia de una capa con inclinación y/o anisotropía teniendo en cuenta entre otras cosas, los cambios de polaridades de las fases presentes en las FRs radiales y transversales. En presencia de anisotropía, se espera observar una diferencia del tiempo de arribo de la conversión Ps en la FR radial y transversal (Peng y Humphrey, 1997). Por otro lado, la presencia de interfases con inclinación genera cambios en las amplitudes de las fases convertidas Ps respecto al azimut alrededor del punto de observación y además genera un arribo directo en la componente transversal (Megna y Morelli, 1994). Algunos autores determinan el strike de inclinación de las capas considerando los cambios de polaridad teniendo en cuenta la forma de onda de la fase P original (Owens et al., 1988; Zhang y Langston, 1995).

Si se considera una fase Ps que se propaga por un medio anisótropo, ésta se separará en dos fases: una fase rápida y una fase lenta que se irán separando en tiempo entre sí a medida que las ondas se propagan. Estas dos fases proyectadas en la componente radial concentran mayor energía. Mientras que si se elimina el efecto de las mismas en la componente transversal, la energía de dicha componente disminuirá (Figura 2.16). En la práctica y en presencia de datos de campo, la característica más significativa que produce la anisotropía es la diferencia en tiempos de arribo de las fases convertidas en la componente radial y transversal.

Por otro lado, si se considera una onda P que en su propagación incide en una interfase con inclinación, se generarán conversiones en ambas componentes de la FR, pero llegarán con el mismo tiempo de arribo y frecuencia. En este caso se observa un cambio de polaridad de la fase Ps en la componente transversal respecto de la onda P directa exhibida por la componente radial. La característica más significativa en el caso de presencia de interfases con inclinación son los cambios de polaridad de las fases en las componentes radial y transversal.

La presencia de interfases con inclinación (Langston, 1977; Zhang y Langston, 1995) y los efectos de anisotropía (McNamara y Owens, 1993), pueden ser analizados y distinguidos con la técnica de FRs si se consideran las componentes transversales para distintos BAZ.

Con este fin, se calcularon las FRs transversales en la estación USHA (Figura 2.17). En general, las diferencias en el tiempo de arribo de la fase Ps en la componente radial y transversal indican la presencia de anisotropía. A pesar de no observar estas diferencias de tiempos de arribo en el caso de USHA, sí se observa energía en las componentes transversales y un cambio de polaridad de ciertas fases sugiriendo en este caso, la presencia de capas con inclinación. Considerando las amplitudes de las fases y sus polaridades en las componentes radiales y transversales es posible estimar la 
dirección y magnitud de inclinación de una interfase.



Figura 2.16: Ejemplo del resultado de FRs sintéticas obtenidas a partir de un modelo con presencia de anisotropía y otro con una Moho con inclinación. La fase convertida Ps se observa a los $3 \mathrm{~s}$. N indica el norte y coincide con la dirección de inclinación y con la dirección de anisotropía. R y T son las componentes radial y transversal de las FRs, respectivamente. Se observa el cambio de polaridad de las fases dependiendo del

BAZ en el caso de una Moho con inclinación. (Extraído y modificado de Peng y Humphreys, 1997).

Por otro lado, hay que tener presente que las FRs transversales presentan gran inestabilidad en presencia de ruido además que son sensibles a los efectos de estructuras superficiales (Langston, 1981). Por este motivo, el presente estudio se limitó al análisis de las FRs radiales, mientras que las componentes transversales sólo se consideraron para el caso de la estación USHA. En la Figura 2.17 se observan las FRs radiales y transversales obtenidas en la estación USHA, luego de realizar la selección en función de la calidad de las mismas. En dicha Figura, y en la Figura 2.13 (sección 2.5.2) se observa una fase posterior al primer arribo alrededor de los 1.3-1.5 s con un cambio de polaridad en función del BAZ. Además las FRs transversales contienen una notable energía lo que indica que la estructura en esta zona no es simple. 

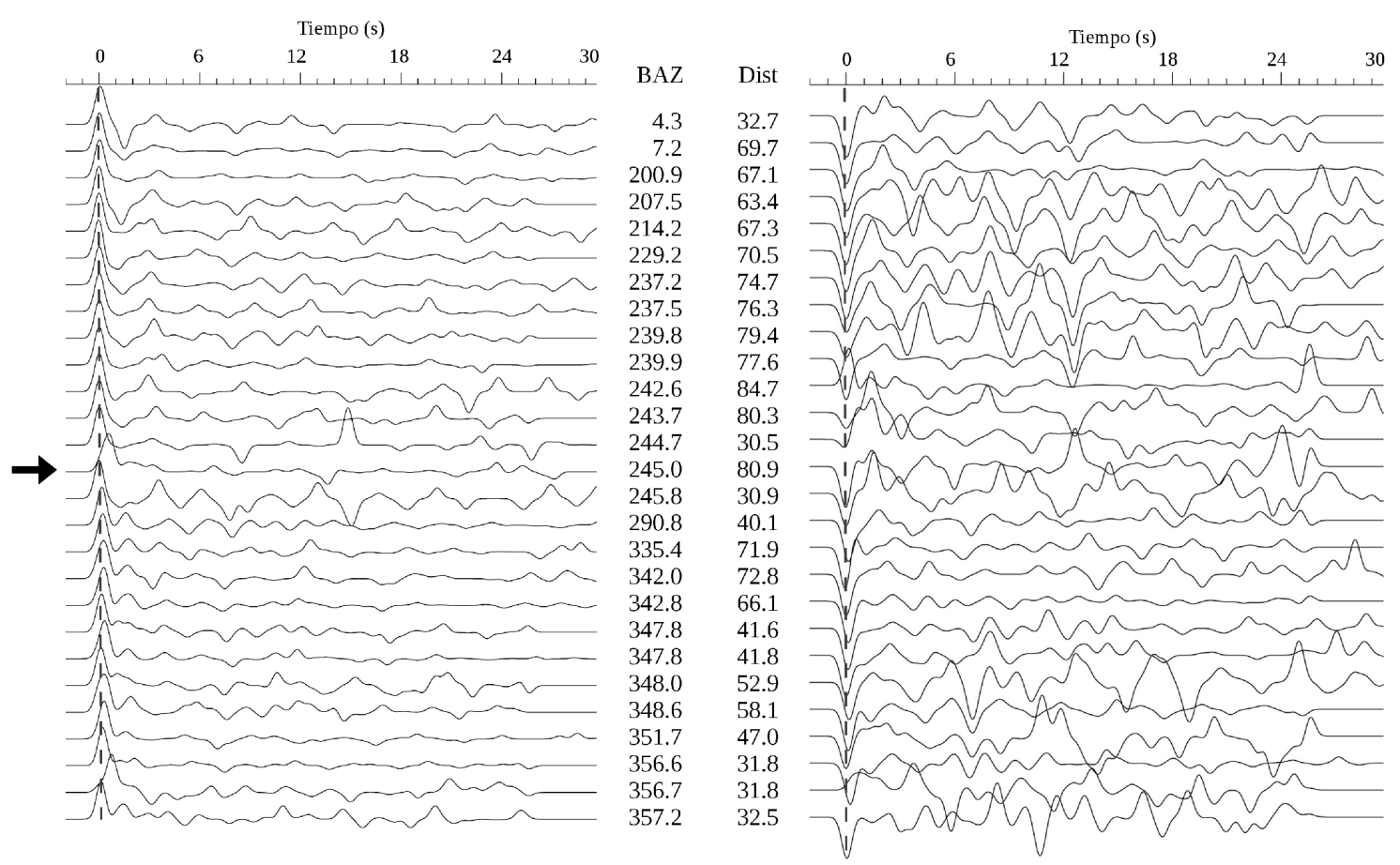

Figura 2.17: FRs radiales (izquierda) y transversales (derecha) seleccionadas en la estación USHA. Se observa la información de BAZ y distancia epicentral (Dist) de los telesismos. La línea punteada indica el primer arribo en ambos casos. Las FR transversales muestran en la mayoría de los casos un primer arribo negativo. La flecha negra indica el BAZ donde se comienza a ver un cambio de polaridad de la fase con tiempo de arribo entre 1.3 y $1.5 \mathrm{~s}$ luego del primer arribo de la onda P.

En el caso particular de la estación USHA pareciera que la observación es mejor explicada por la presencia de una capa con inclinación. Para verificar esta hipótesis, se calcularon las FRs sintéticas basadas en la teoría de rayos de Frederiksen y Bostock (2000). El modelo considerado consiste en 2 capas sobre el manto superior. La profundidad de la Moho se estimó a los $32 \mathrm{~km}$ y se consideró una capa a $5 \mathrm{~km}$ de profundidad con una inclinación de $30^{\circ} \mathrm{NE}$ (strike $280^{\circ}$ ). Luego de varias pruebas preliminares, las velocidades de onda P y S para las 3 capas consideradas fueron: $\mathrm{Vs}_{1}=3.5 \mathrm{~km} / \mathrm{s}, \mathrm{Vp}_{1}=6.3 \mathrm{~km} / \mathrm{s}, \mathrm{Vs}_{2}=3.9 \mathrm{~km} / \mathrm{s}, \mathrm{Vp}_{2}=7 \mathrm{~km} / \mathrm{s}, \mathrm{Vs}_{3}=4.4 \mathrm{~km} / \mathrm{s}$ y $\mathrm{Vp}_{3}=8 \mathrm{~km} / \mathrm{s}$. Se calcularon las FRs sintéticas considerando un BAZ de $240^{\circ}$ y $360^{\circ}$. La comparación entre las FRs sintéticas y las obtenidas a partir de los datos registrados en USHA, muestra un cambio de polaridad similar (Figura 2.18). En la Figura 2.18 se observa el apilamiento de las FRs radiales (en negro) y transversales (en rojo) para los grupos 1 y 2 . El cambio de polaridad se indica con una flecha. Además las FRs transversales sintéticas también muestran una disminución de amplitud alrededor de 1.3 - 1.5 s como en el caso de las FRs obtenidas en USHA. Para hacer un mejor modelado se requerirían más datos con una mejor cobertura azimutal para muestrear la interfase en 
diferentes lugares y poder disminuir las ambigüedades en los modelos finales obtenidos. Desafortunadamente esto no se puede llevar a cabo debido a la presencia de ruido sísmico en los datos de TdF y el gran número de FRs calculadas y descartadas por tener mala calidad, lo que se traduce en una escasa cobertura azimutal. Simplemente con este ejemplo se quiere demostrar que en el caso de USHA, los datos observados estarían sugiriendo la presencia de una capa con inclinación y por consiguiente, los resultados obtenidos deberían considerarse con cautela teniendo presente que la técnica de FRs considera un modelo de capas horizontales sin buzamiento. De todas maneras, no se puede descartar que la estructura esté compuesta por capas inclinadas y estructuras anisótropas. La presencia de capas inclinadas es esperada por observar energía de la onda P en las componentes transversales, algo que no se espera para estructuras planas sin buzamiento con anisotropía azimutal de onda S (Figuras 2.16 y 2.17).
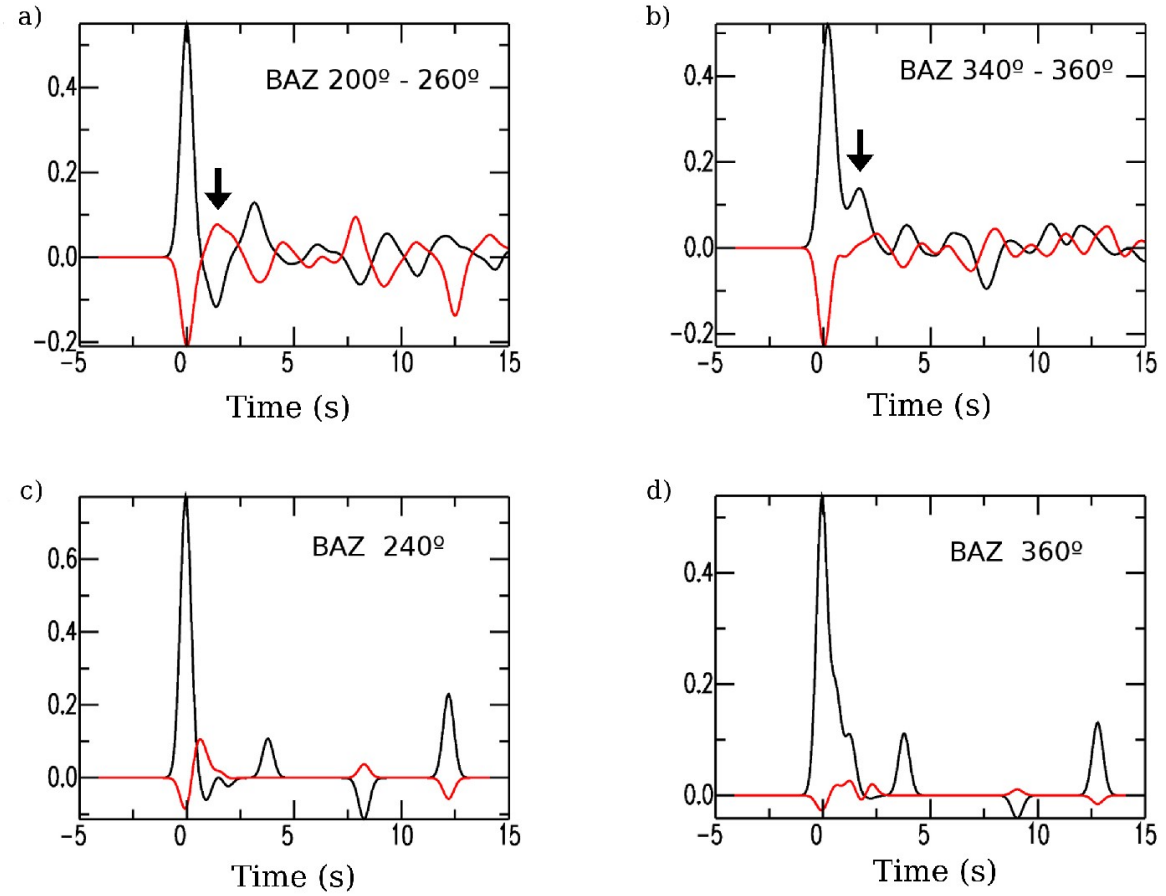

Figura 2.18: apilamiento de las FRs radiales (negro) y transversales (Rojo) obtenidas en USHA con un BAZ de a) $200^{\circ}-260^{\circ}$ y b) $340^{\circ}-360^{\circ}$. FRs sintéticas c) BAZ $240^{\circ}$ y d) BAZ $360^{\circ}$, calculadas para una Moho con una profundidad de $32 \mathrm{~km}$ y una discontinuidad a $5 \mathrm{~km}$ de profundidad inclinada $30^{\circ}$ al NE. Las flechas negras indican el cambio de polaridad de la fase en cuestión observada entre 1.3 y 1.5 s. 


\section{Apilamiento de las Funciones del Receptor:}

Método de H-k stacking

\subsection{Estimación del espesor cortical y de la relación $V_{\mathrm{P}} / \mathrm{V}_{\mathrm{S}}$}

La Moho que determina el límite entre corteza y manto superior, representa un cambio en las velocidades sísmicas así como también cambios en la composición química y en la reología. Para caracterizar la estructura cortical de una región es importante estimar la profundidad de la Moho o espesor cortical (H), la cual está relacionada con la geología y con la evolución tectónica del área de estudio. Durante las últimas décadas, se han llevado a cabo numerosos estudios con el fin de obtener información de la estructura cortical en diferentes regiones utilizando la técnica de las FRs descripta en el Capítulo II (Langston, 1977; Zandt y Ammon, 1995; Zhu y Kanamori, 2000; Zhu, 2004). La conversión Ps y sus múltiples PpPs y PpSs+PsPs contienen información de la profundidad de la Moho y de la relación de velocidades sísmicas $\mathrm{V}_{\mathrm{p}} / \mathrm{V}_{\mathrm{s}}$. Para calcular dicha profundidad, es necesario convertir el tiempo de arribo de la fase Ps a profundidad y para eso se requiere información previa de las velocidades sísmicas que muchas veces se aproximan por un perfil simple en velocidad de ondas $\mathrm{P}$ y de la relación de velocidades sísmicas $\mathrm{V}_{\mathrm{P}} / \mathrm{V}_{\mathrm{s}}$. Pero si no se dispone de dicha información, es posible estimar $\mathrm{H}$ y $\mathrm{V}_{\mathrm{P}} / \mathrm{V}_{\mathrm{S}}$ considerando además los tiempos de arribo de las fases múltiples o reverberaciones.

Si se considera velocidades de corteza promedio para las ondas $\mathrm{P}$ y $\mathrm{S}$, es posible estimar el espesor cortical teniendo en cuenta la diferencia de tiempos de arribo de la onda P directa y de la fase convertida Ps de la siguiente manera (Zhu y Kanamori, 2000):

$$
H=\frac{t_{P s}}{\sqrt{\frac{1}{V_{s}^{2}}-p^{2}}-\sqrt{\frac{1}{V_{p}^{2}}-p^{2}}}
$$

siendo $p$ el parámetro del rayo sísmico de la onda incidente. 
Un problema que existe es el trade-off entre el espesor y las velocidades sísmicas. Sin embargo, ya que $t_{\mathrm{Ps}}$ representa la diferencia del tiempo de viaje de la onda $\mathrm{S}$ respecto de la onda $\mathrm{P}$ en la corteza, la dependencia de $\mathrm{H}$ con $\mathrm{V}_{\mathrm{P}}$ no es tan significativacomo con $\mathrm{V}_{\mathrm{S}}$ (o más precisamente con la relación de velocidades sísmicas que denominamos k). Esto se puede demostrar con el siguiente ejemplo numérico:

Si se considera una velocidad media $V_{\mathrm{P}}=6.3 \mathrm{~km} / \mathrm{s}$ y $\mathrm{k}=1.732$, para un espesor cortical de $30 \mathrm{~km}$ se obtiene:

$$
\Delta H=\frac{d H}{d V_{p}} \Delta V_{p}=4.3 \Delta V_{p}(\mathrm{~km})
$$

Así la incertidumbre de $\mathrm{H}$ es $<0.5 \mathrm{~km}$ si se considera $0.1 \mathrm{~km} / \mathrm{s}$ de incertidumbre en $\mathrm{V}_{\mathrm{p}}$. Sin embargo el espesor $\mathrm{H}$ es muy dependiente de la relación de velocidades sísmicas $\mathrm{V}_{\mathrm{P}} / \mathrm{V}_{\mathrm{S}}$ :

$$
\Delta H=\frac{d H}{d k} \Delta k=-40.2 \Delta k(k m)
$$

es decir, si el valor de k varía en 0.1, esto se traduce en un cambio de $4 \mathrm{~km}$ en el espesor cortical (Zhu y Kanamori, 2000).

Una manera de reducir esta ambigüedad es utilizar las fases múltiples que se observan después de la llegada de la conversión Ps:

$$
\begin{gathered}
H=\frac{t_{P p P s}}{\sqrt{\frac{1}{V_{s}^{2}}-p^{2}}+\sqrt{\frac{1}{V_{p}^{2}}-p^{2}}} \\
H=\frac{t_{P p S s+P S P s}}{2 \cdot \sqrt{\frac{1}{V_{s}^{2}}-p^{2}}}
\end{gathered}
$$

De esta manera, conociendo el parámetro del rayo sísmico, los tiempos de propagación de las conversiones presentes en una FR y asumiendo una velocidad $V_{P}$, es entonces posible estimar $\mathrm{H}$ y $\mathrm{V}_{\mathrm{P}} / \mathrm{V}_{\mathrm{S}}$ a partir de las ecuaciones anteriores (Zhu, 1993; Zandt y Ammon, 1995).

\subsection{Apilamiento de las FRs}

Cuando las FRs son de muy buena calidad, basta con identificar los arribos de las 
reverberaciones y estimar el espesor cortical y la relación de velocidades sísmicas. Sin embargo, en muchas regiones la complejidad de los ambientes tectónicos y geológicos se ven reflejados en formas de ondas a partir de las cuales la identificación de las fases en una FR es más complicada como es el caso de TdF. Además generalmente se trabaja con telesismos de diferentes magnitudes, profundidades y BAZ por lo que la propagación de las ondas en cada caso puede ser muy diferente y la SNR no es buena. En estos casos se suele utilizar la técnica de apilamiento (o stacking en inglés) de las FRs que consiste en promediar todas las FRs obtenidas y así mejorar la SNR.

Las ventajas de apilar las FRs pueden resumirse en que:

reduce significativamente el ruido sísmico

$>$ realza la presencia de fases coherentes

$>$ aumenta la SNR

\subsubsection{Método de H-k stacking}

Cuando una señal telesísmica es registrada en una estación, ésta viene acompañada de ruido sísmico ambiental, scattering por heterogeneidades corticales y conversiones de onda $\mathrm{P}$ a $\mathrm{S}$ relacionadas con otras discontinuidades de velocidades sísmicas diferentes a la Moho. Debido a esto, a menudo es complicado identificar la conversión Ps y sus múltiples debidas a la Moho presentes en una FR. Para favorecer la identificación de las conversiones, Zhu y Kanamori (2000) propusieron un algoritmo que estima el espesor cortical considerando el apilamiento de las FRs. Esta técnica es conocida como "H-k stacking" y ha sido aplicada en numerosos trabajos para investigar la estructura cortical en diferentes regiones (Zhu y Kanamori, 2000; Ai et al., 2005; Tomlinson et al., 2006; Lombardi et al., 2008; Piana Agostinetti y Amato, 2009; Thompson et al., 2010; Wang et al., 2010; Akpan et al., 2016).

El concepto de esta técnica se basa en generar un modelo de capas horizontales para cada par de valores de espesor cortical y relación de velocidades sísmicas $(\mathrm{H}, \mathrm{k})$ y calcular los tiempos de arribo de las fases relativas a la onda $\mathrm{P}$ directa correspondiente a un parámetro de rayo conocido. Conociendo esos tiempos de arribo el stack s(H-k) está dado por la ecuación:

$$
s(H, k)=w_{1} r\left(t_{1}\right)+w_{2} r\left(t_{2}\right)-w_{3} r\left(t_{3}\right)
$$

siendo $r(t)$ la FR radial, $w_{i}$ los factores de pesos de cada fase, $t_{1}, t_{2}$ y $t_{3}$ los tiempos de arribo 
calculados de las fases Ps, PpPs y PpSs+PsPs correspondientes a un valor de $\mathrm{H}$ y de k. El signo negativo en el arribo de la fase PpSs+PsPs se debe a la polaridad inversa esperada respecto de los otros arribos. El stack $\mathrm{s}(\mathrm{H}, \mathrm{k})$ alcanza el valor máximo cuando las tres fases son sumadas constructivamente con el valor correcto de $\mathrm{H}$ y k (Figura 3.1).

La aplicación de esta técnica requiere de un valor previo de velocidad $\mathrm{V}_{\mathrm{P}}$. Un cambio de 0.1 $\mathrm{km} / \mathrm{s}$ del valor de esa velocidad inicial corresponde a un cambio en el espesor cortical de $0.5 \mathrm{~km}$ (Zhu y Kanamori, 2000), lo que demuestra que la técnica depende de cierto conocimiento previo de velocidades. La ventaja de este método es que no es necesario picar tiempos de arribo. La Figura 3.1 muestra las curvas que representan la contribución de la conversión directa Ps y de sus dos múltiples principales PpPs y PpSs+PsPs a las amplitudes sumadas como una función de espesor cortical y relación de velocidades sísmicas.

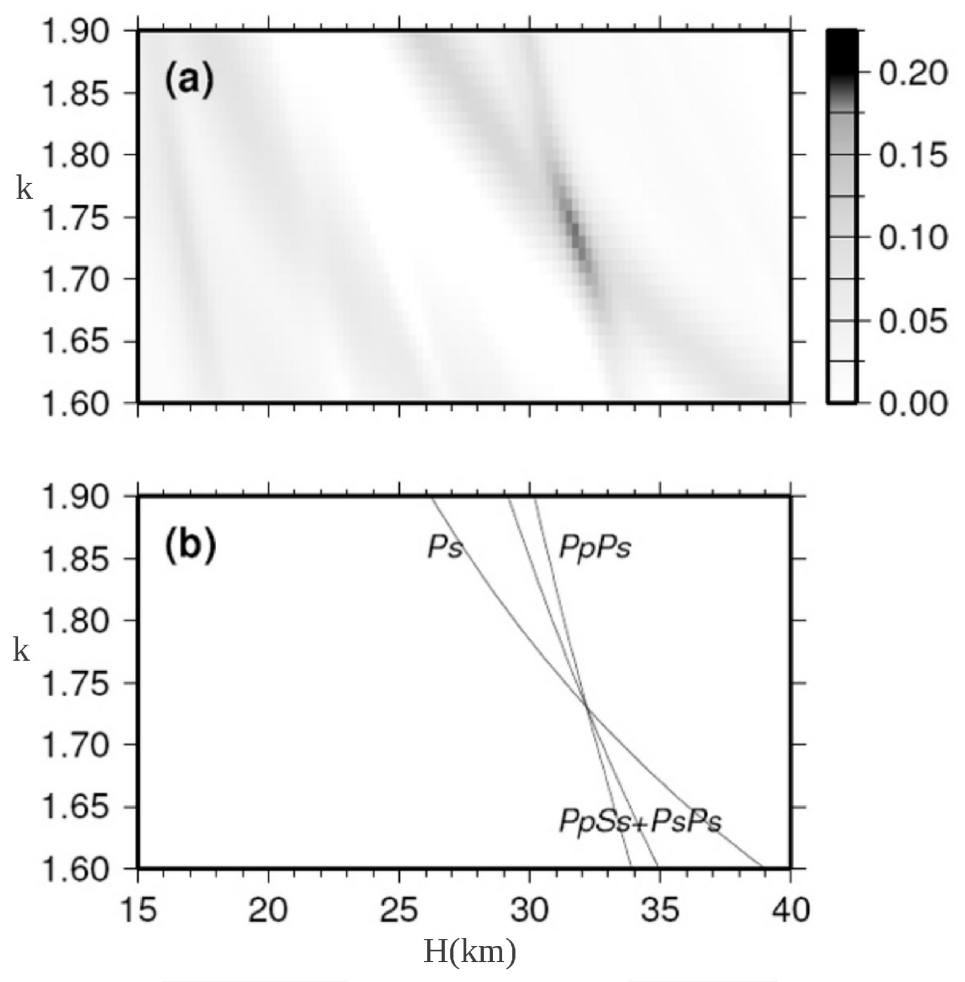

Figura 3.1: a) Resultado del stack s(H,k) de las FRs que alcanza el valor máximo en la zona más oscura cuando el valor de $\mathrm{H}$ y $\mathrm{V}_{\mathrm{P}} / \mathrm{V}_{\mathrm{s}}$ utilizados en el stack son los correctos. b) Relación entre $\mathrm{H}$ y k para las diferentes fases convertidas. Cada línea representa la contribución al apilamiento de la fase Ps y sus múltiples. (Extraído y modificado de Zhu y Kanamori, 2000). 


\subsubsection{Selección de factores de peso $w_{i}$}

Para poder aplicar la técnica de H-k stacking es necesario seleccionar ciertos parámetros iniciales como la velocidad $\mathrm{V}_{\mathrm{P}}$ y los factores de pesos $\mathrm{w}_{\mathrm{i}}$ de las fases convertidas. Zhu y Kanamori (2000) seleccionaron $\mathrm{w}_{1}=0.7, \mathrm{w}_{2}=0.2 \mathrm{y} \mathrm{w}_{3}=0.1$, siendo $\mathrm{w}_{1}, \mathrm{w}_{2} \mathrm{y} \mathrm{w}_{3}$ los pesos de las fases Ps, PpPs y PpSs+PsPs, respectivamente. Su criterio de selección se basa en el hecho de que la fase Ps es la que generalmente se observa con mayor amplitud y la que más contribuye en la FR en comparación con las fases múltiples. La selección de estos factores de peso es arbitraria y dependerá de cada problema en particular, teniendo en cuenta la calidad de los datos, la SNR como también la identificación visual de la conversión Ps y sus reverberaciones.

En algunos casos una pequeña variación en los factores de peso puede dar un resultado muy diferente (Figura 3.2), lo que demuestra que la solución del problema no es única y que se requiere información previa de la estructura para poder discernir entre una solución y otra.
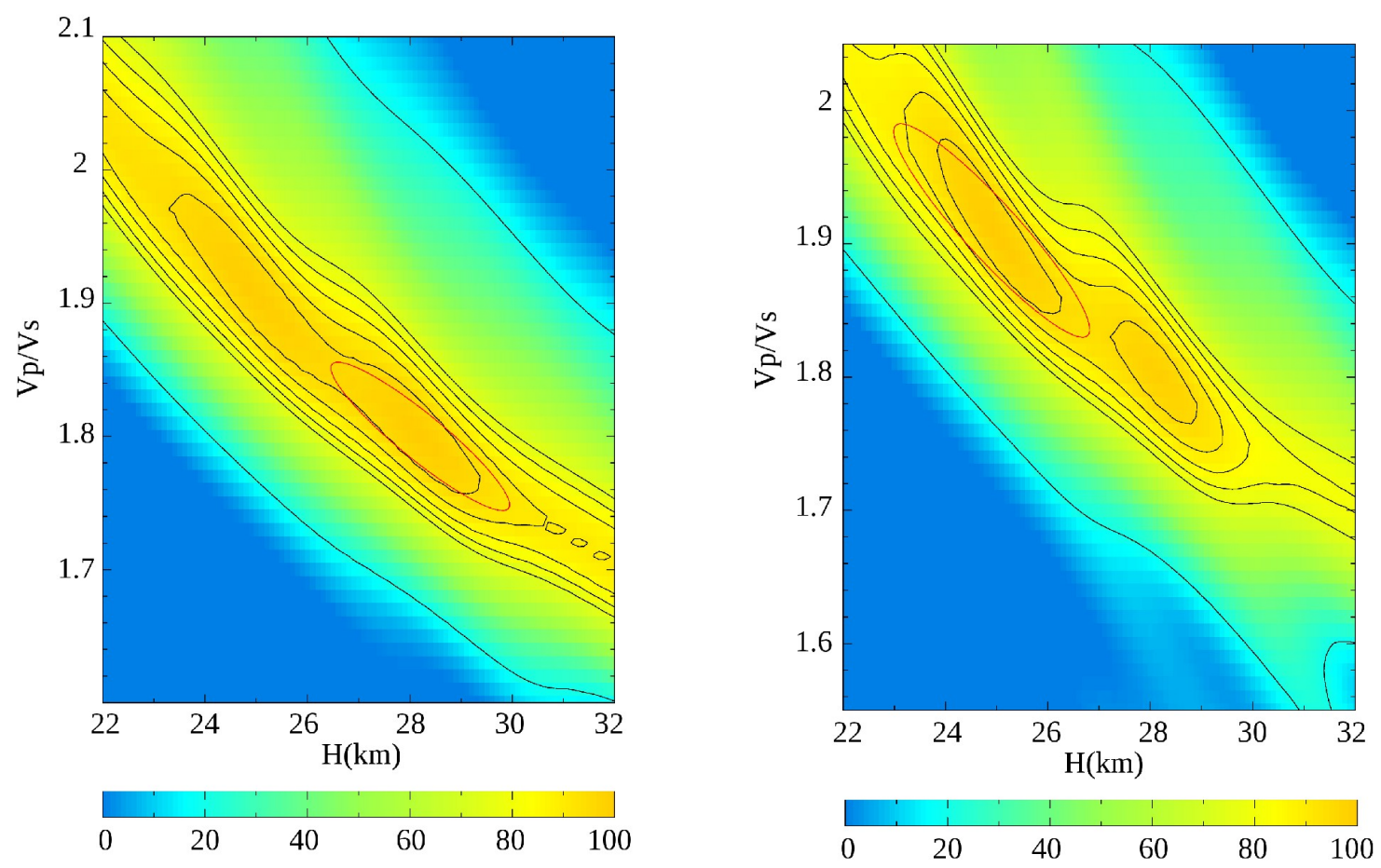

Figura 3.2: Ejemplo de aplicar la técnica de H-k stacking en la estación ELCA con pesos: izq.) w1=0.7, w2=0.2 y w3=0.1; der.) w1=0.5, w2=0.3 y w3=0.2. En ambos casos se utilizaron las mismas FRs y velocidad


en el apilamiento. Los círculos rojos indican en cada caso el máximo valor del stack s(H,k). 
Con el fin de calcular el apilamento de las FRs obtenidas en TdF, se propuso un criterio considerando una combinación de los pesos $\mathrm{w}_{\mathrm{i}}$, pero asignando mayor peso relativamente a la fase convertida Ps por ser la que mejor se identificaba. De esta manera fue posible comprobar si los resultados se modificaban por un cambio del valor del factor de peso o si los valores encontrados eran robustos.

El criterio adoptado fue el siguiente:

- $\mathrm{W}_{1}>\mathrm{w}_{2}>\mathrm{w}_{3}$,

- $\mathrm{w}_{1}+\mathrm{w}_{2}+\mathrm{w}_{3}=1$

- $\quad \mathrm{w}_{1} \geq 0.5$

\subsubsection{Estimación de la incertidumbre}

Cuando se aplica la técnica de H-k stacking hay dos métodos para determinar el error en $\mathrm{H}$ y k. El primero estima el error basándose en la curvatura del pico que representa el máximo del stack, siendo el error mayor cuanto más grande sea la curvatura del pico máximo. La segunda forma de estimar el error es mediante el método de bootstrap (Efron y Tibshirani, 1991), que genera un nuevo grupo de FRs mediante un remuestreo aleatorio. Luego realiza el apilamiento de este nuevo grupo y destaca el máximo global. Este procedimiento se repite tantas veces como se quiera para producir un grupo de valores de $\mathrm{H}$ y k en el cual es posible calcular la desviación estándar. Es importante destacar que este valor de desviación estándar tiene un significado diferente en el caso de tener un solo máximo global bien definido en oposición a varios máximos locales, en los cuales ninguno es significativamente diferente del otro. Es decir, en el primer caso, el error refleja el ancho del pico e indica una verdadera incertidumbre en los valores. En el segundo caso, el máximo global de cada bootstrap individual puede corresponder a un máximo local diferente e indica que puede haber varias interpretaciones y el sistema no puede diferenciarlas. En general, pequeños valores de la desviación estándar indican un solo máximo presente, y grandes valores indican la presencia de múltiples máximos locales similares. De todas formas, la desviación estándar no es estrictamente una medida de error sino una medida de la robustez de los valores $\mathrm{H}$ y $\mathrm{k}$ con respecto a variaciones en el grupo de datos. Asimismo, una alta robustez generalmente indica un resultado estable, pero que puede tener un error debido a la elección de la velocidad promedio $V_{\mathrm{p}}$.

El método de bootstrap es una buena manera de caracterizar y controlar el resultado ya que contribuyen tanto el ancho del pico máximo como la existencia de múltiples máximos similares. La existencia de múltiples máximos representa un problema al momento de determinar $\mathrm{H}$ y $\mathrm{k}$ automáticamente pero puede ser considerado como una fuente de información. Es decir, la presencia de varios máximos locales indica que la estructura es más compleja que un modelo 
compuesto por simples capas horizontales y deberían ser analizados con mayor detalle.

\subsection{Aplicación del método en TdF}

\subsubsection{Procedimiento}

Para aplicar el método de H-k stacking, las FRs obtenidas en TdF fueron seleccionadas manualmente de manera de utilizar sólo aquellas que incluyeran una conversión Ps visible y un pulso positivo a los 0 s que corresponde al arribo directo de la onda P. Luego de dicha selección (ver Figura 2.7 del Capítulo II), se observó que los eventos seleccionados están ubicados básicamente en el SO y $\mathrm{N}$ de TdF y que las formas de onda de las FRs en cada estación varían dependiendo del BAZ del telesismo. Por otro lado, con el fin de determinar el criterio de selección de pesos definido en la sección 3.2.1, se realizaron varias pruebas en cada estación teniendo en cuenta todas las FR seleccionadas sin separar por BAZ. Estos resultados preliminares demostraron que los valores de $\mathrm{H}$ y k en algunos casos eran ambiguos y se observó la presencia de varios máximos locales o soluciones posibles en algunos casos. Un ejemplo de esto se muestra en la Figura 3.2 cuando se realizaron las pruebas con datos de la estación ELCA. Teniendo en cuenta esto, y que además se observó una variabilidad de la forma de ondas de las FRs en función del BAZ, para el posterior análisis, los datos fueron separados en dos grupos: grupo 1 (eventos provenientes del SO) y grupo 2 (eventos provenientes del N) como se mencionó en las secciones 2.5.1 a 2.5.4 del Capítulo II. Esta separación se basó en los puntos de conversión de los telesismos que generaron las FRs seleccionadas (Figura 3.3).

Dichos puntos también conocidos como piercing points en inglés, son puntos en los que la onda P se convierte en S, cuya localización es calculada a partir del tiempo de propagación entre la estación y la fuente del telesismo y considerando un valor de espesor cortical medio de $30 \mathrm{~km}$ en este trabajo. En el caso de la estación ELCA, debido al menor tiempo de observación por ser la última estación instalada, la cantidad de eventos fue menor en comparación con el resto de las estaciones obteniéndose desafortunadamente un escaso número de FRs de buena calidad correspondientes a eventos situados al SO de TdF.

Según el criterio adoptado en la sección 3.2.1 en relación a la selección de factores de pesos, se aplicó el método de $\mathrm{H}-\mathrm{k}$ stacking, obteniendo 8 soluciones de $\mathrm{H}$ y k para cada grupo en cada estación teniendo en cuenta todas las posibles combinaciones de factores de pesos para la conversión Ps y sus múltiples. Se asumió una velocidad de corteza promedio de $\mathrm{V}_{\mathrm{P}}=6.1 \mathrm{~km} / \mathrm{s}$ basado en el modelo obtenido por Lawrence y Wiens (2004) en la estación sísmica VTDF localizado en la Estancia Vicuña, Tierra del Fuego $\left(-54.14^{\circ},-68.71^{\circ}\right)$. Además se aplicó el H-k stacking conjuntamente con un algoritmo de bootstrap de 200 repeticiones. 


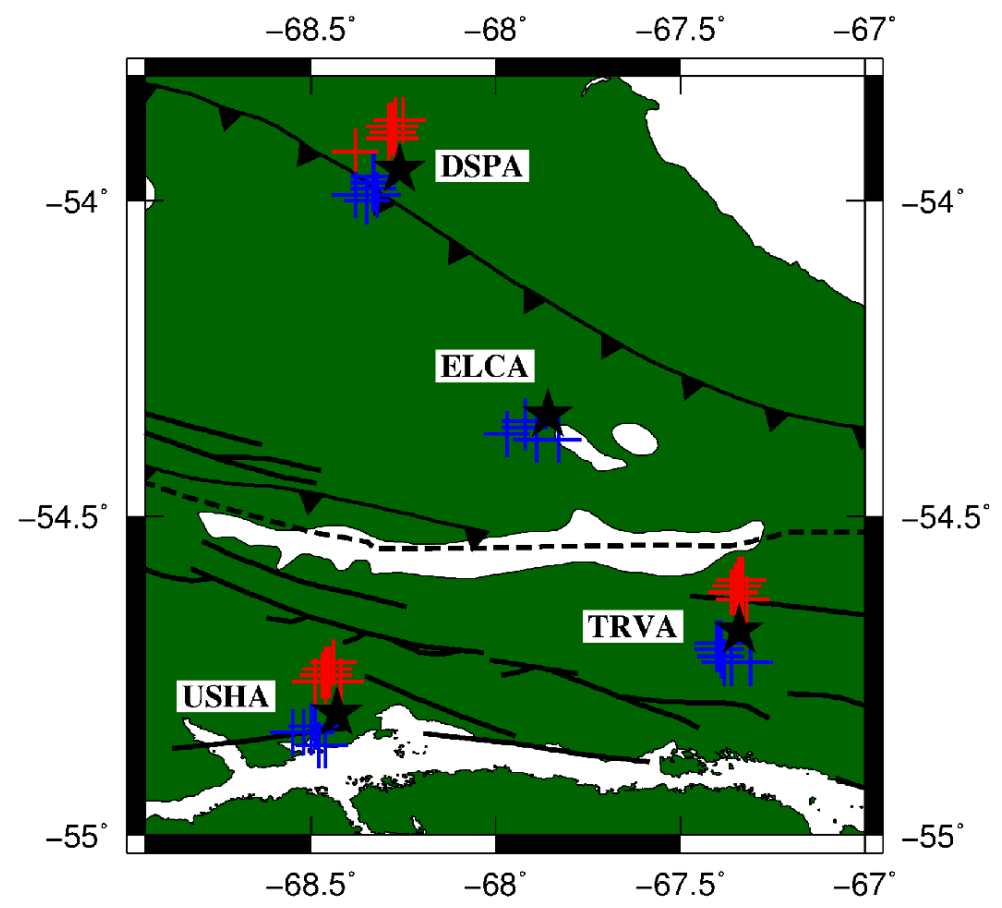

Figura 3.3: Ubicación de los puntos de conversión de los telesismos que generaron las FRs seleccionadas: grupo 1 (eventos al SO de la estación) en azul y grupo 2 (eventos al N de la estación) en rojo. Para la localización de los puntos de conversión se asumió una Moho a 30 km de profundidad.

\subsubsection{Resultados y conclusiones}

En las Figuras 3.4 a 3.7 se observa el resultado de aplicar el método a cada grupo de cada estación considerando el caso particular cuando los factores de peso son: $\mathrm{w}_{1}=0.7, \mathrm{w}_{2}=0.2$ y w $\mathrm{w}_{3}=$ 0.1. En algunos casos se observan otros máximos o soluciones posibles, pero cuando se aplica el bootstrap el resultado final siempre gira en torno a los mismos valores, independientemente de los factores de pesos utilizados.

Si bien el grupo 1 y 2 de la estación TRVA y el grupo 1 de la estación USHA son los que se encuentran cercanos a fallas geológicas, demostraron tener resultados más robustos y con menor variación. Al contrario, el grupo 1 de la estación DSPA y ELCA es el que dio resultados de $\mathrm{H}$ y $\mathrm{V}_{\mathrm{P}} / \mathrm{V}_{\mathrm{S}}$ con mayor error. Dicho grupo corresponde a eventos ubicados al SO de las respectivas estaciones. Esto podría deberse a que se encuentran ubicadas en el límite de diferentes unidades geológicas. La presencia significativa de sedimentos puede alterar la conversión Ps y complicar la estimación de la profundidad de la Moho. 


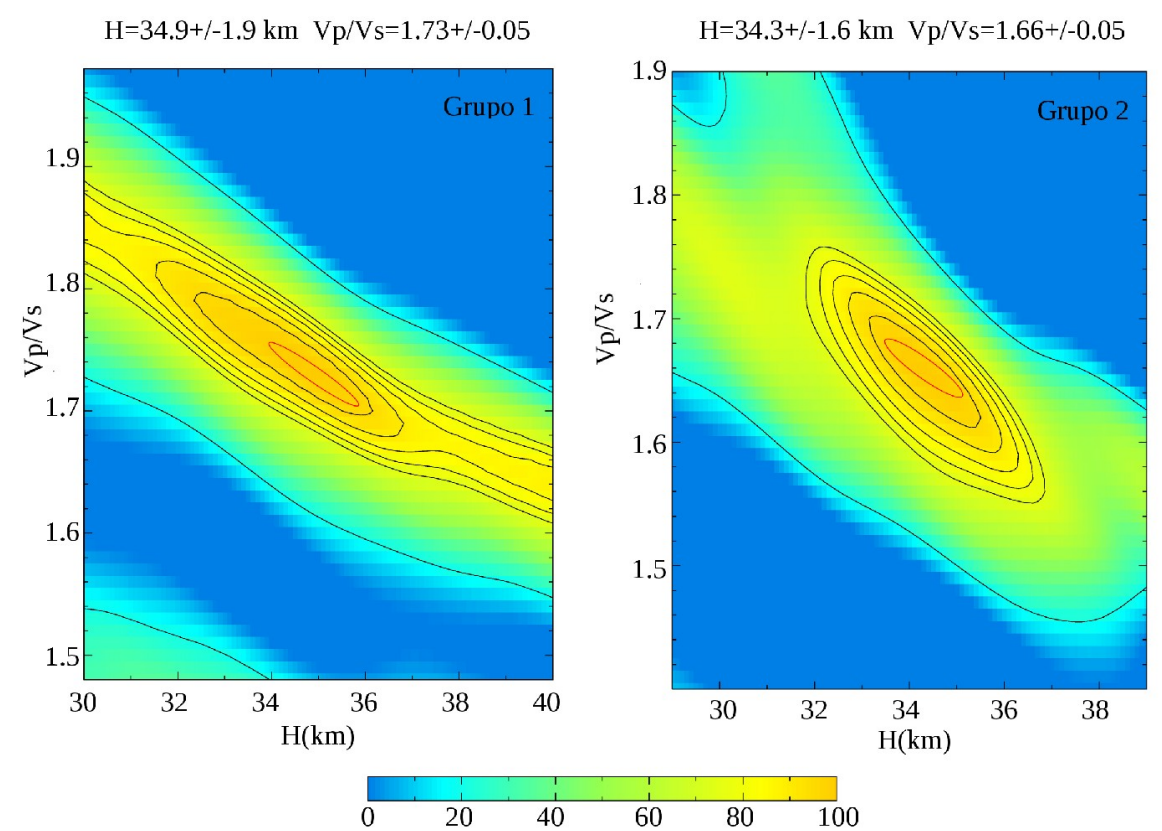

Figura 3.4: Resultados obtenidos en la estación TRVA para el grupo 1 (izquierda, eventos al SO de la estación) y grupo 2 (derecha, eventos al $\mathrm{N}$ de la estación) considerando los factores de pesos $\mathrm{w}_{1}=0.7, \mathrm{w}_{2}=$ 0.2 y w $_{3}=0.3$. Los valores +/- son la desviación estándar obtenidos por bootstrap.

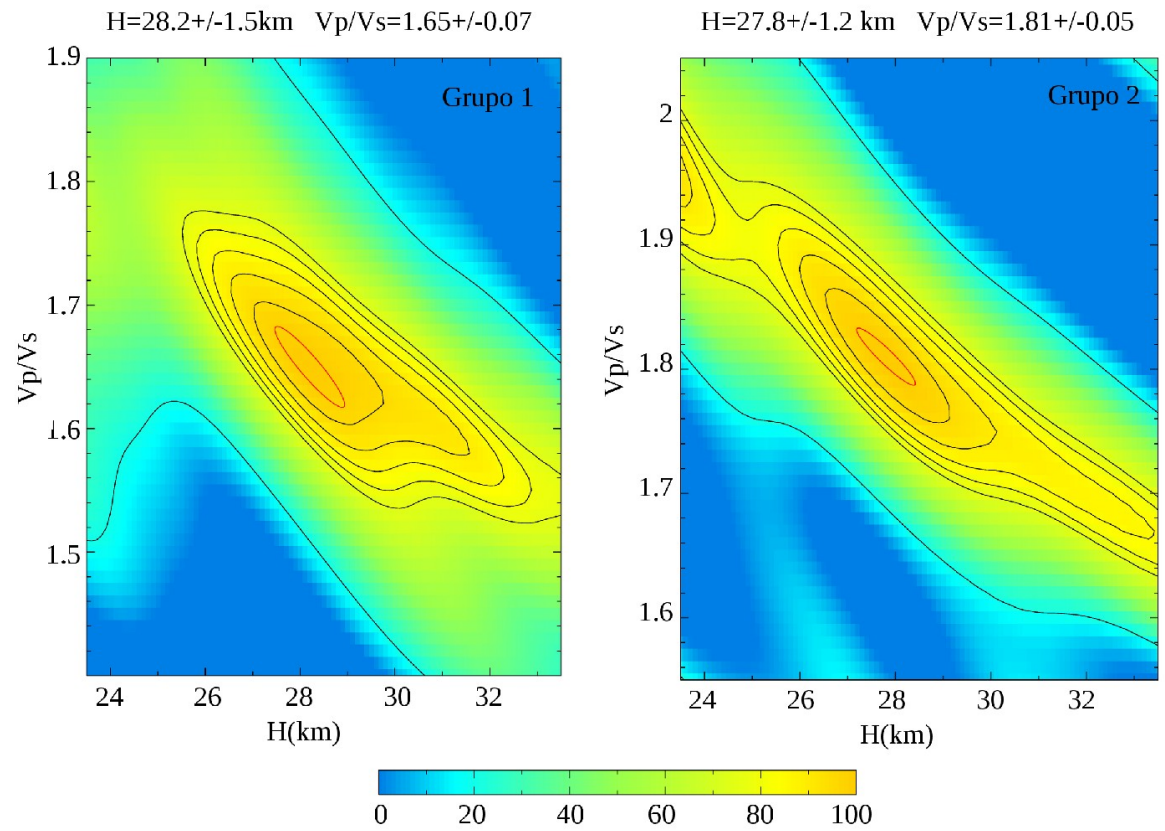

Figura 3.5 : Resultados obtenidos en la estación USHA para el grupo 1 (izquierda, eventos al SO de la estación) y grupo 2 (derecha, eventos al $\mathrm{N}$ de la estación) considerando los factores de pesos $\mathrm{w}_{1}=0.7, \mathrm{w}_{2}=$ 0.2 y w $_{3}=0.3$. Los valores +/- son la desviación estándar obtenidos por bootstrap. 


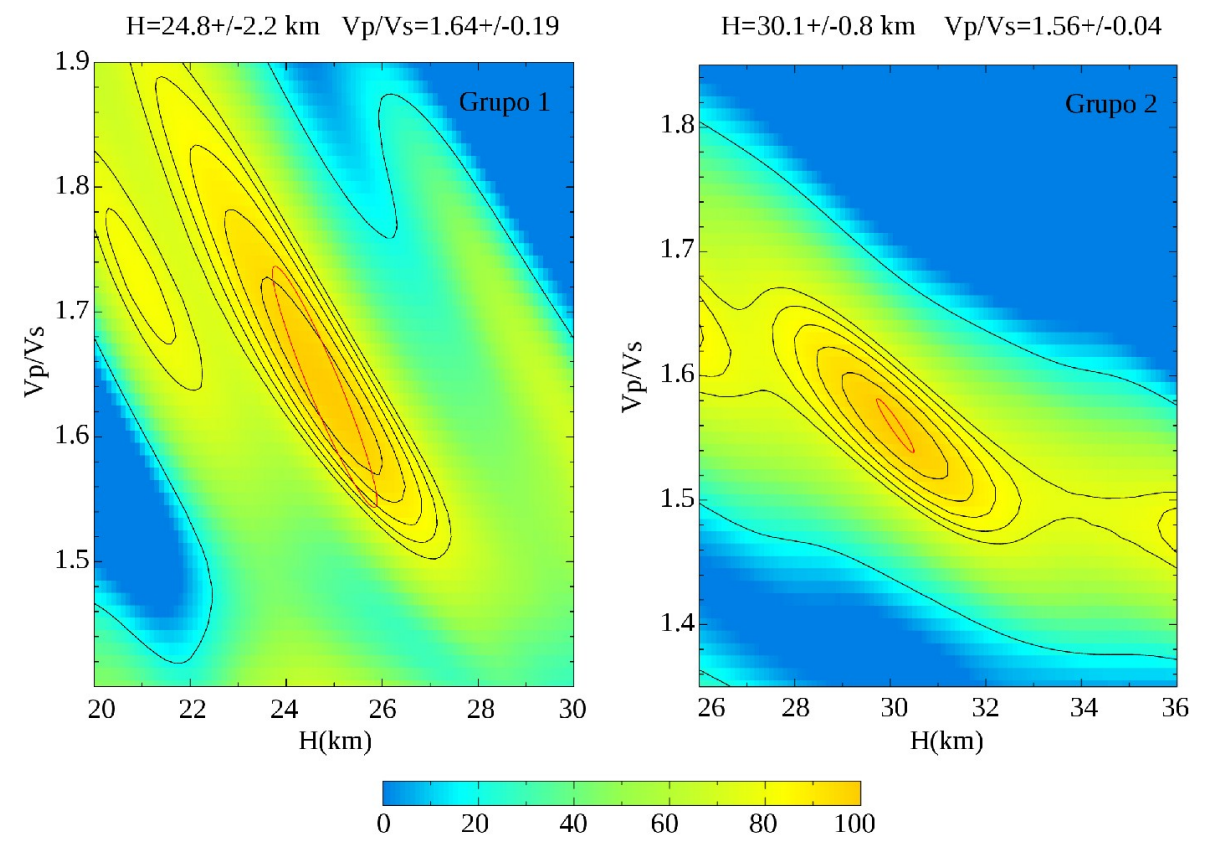

Figura 3.6: Resultados obtenidos en la estación DSPA para el grupo 1 (izquierda, eventos al SO de la estación) y grupo 2 (derecha, eventos al $\mathrm{N}$ de la estación) considerando los factores de pesos $\mathrm{w}_{1}=0.7, \mathrm{w}_{2}=$ 0.2 y w $_{3}=0.3$. Los valores +/- son la desviación estándar obtenidos por bootstrap.

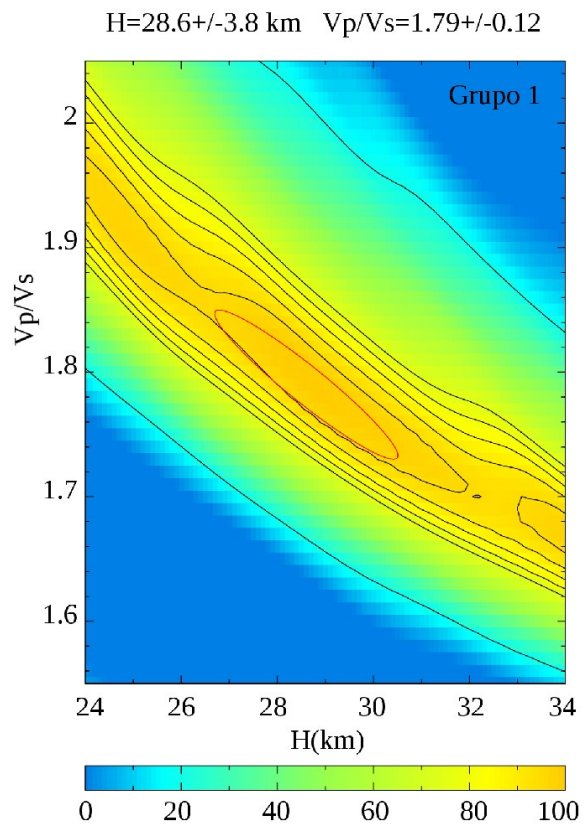

Figura 3.7 : Resultados obtenidos en la estación ELCA para el grupo 1 (eventos al SO de la estación) considerando los factores de pesos $\mathrm{w}_{1}=0.7, \mathrm{w}_{2}=0.2$ y w $\mathrm{w}_{3}=0.3$. Los valores + - son la desviación estándar obtenidos por bootstrap. 
En las Figura 3.8 se observan todas las soluciones posibles cuando se aplican diferentes factores de peso en cada grupo de cada estación. Los valores de espesor cortical y de la relación de velocidades sísmicas $\mathrm{V}_{\mathrm{P}} / \mathrm{V}_{\mathrm{S}}$ están en el rango de 24.8 - $34.8 \mathrm{~km}$ y 1.56 - 1.80, respectivamente dependiendo de la estación, del grupo de puntos de conversión y de la combinación de factores de pesos utilizados. El valor mínimo de espesor cortical fue obtenido en la estación DSPA mientras que el valor máximo se obtuvo en la estación TRVA, sugiriendo un aumento del espesor cortical de la Isla de norte a sur.

Las diferentes soluciones para las diferentes combinaciones de factores de pesos se encuentran alrededor de sus valores medios. En la tabla 3.1 se observa el valor medio de cada solución con su desviación estándar. La desviación estándar demuestra la variación de los resultados con respecto a la variación de los pesos. En todas las estaciones, luego de la separación en los dos grupos, las soluciones obtenidas son robustas respecto a cambios en los factores de pesos lo que da confianza en los resultados presentados.

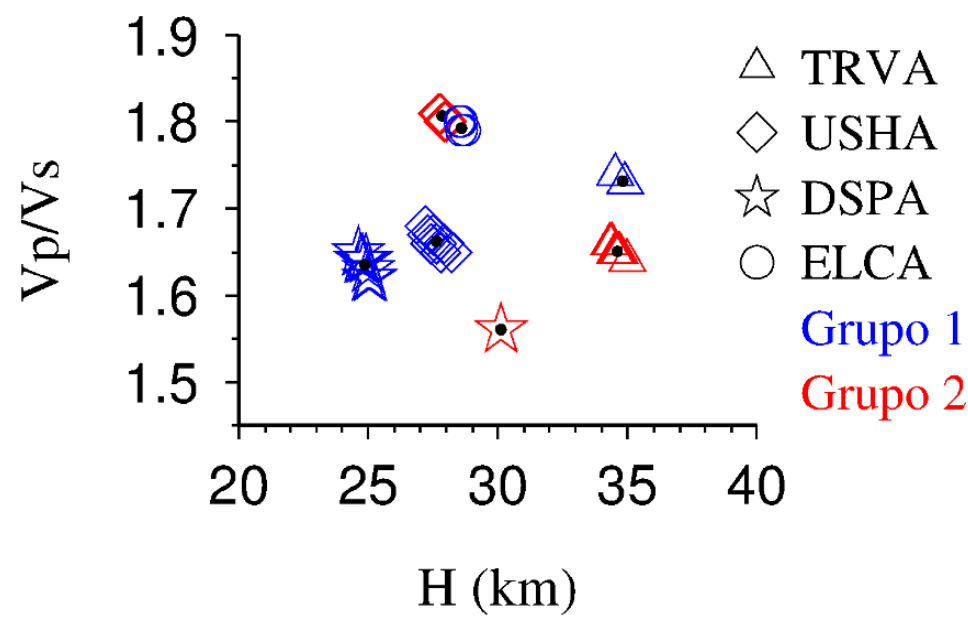

Figura 3.8: Valores de espesor cortical $\mathrm{H}$ y relación de velocidades sísmicas $\mathrm{V}_{\mathrm{P}} / \mathrm{V}_{\mathrm{S}}$ obtenidos en cada grupo de cada estación a partir del método de H-k stacking. Se utilizaron diferentes símbolos y colores para diferenciar las estaciones y grupos de eventos, respectivamente. Grupo 1 representa a los eventos provenientes del SO y grupo 2 a eventos provenientes del N. Los puntos negros representan el valor medio de la profundidad de la Moho $(\mathrm{H})$ y de la relación de velocidades sísmicas $\mathrm{V}_{\mathrm{P}} / \mathrm{V}_{\mathrm{S}}$ obtenido en cada grupo de cada estación. Para cada grupo existen 8 soluciones teniendo en cuenta las diferentes combinaciones de pesos. 


\begin{tabular}{|c|c|c|c|c|}
\hline Estación - Grupo & $\overline{\mathbf{H}}(\mathbf{k m )}$ & $\boldsymbol{\sigma}_{\overline{\mathbf{H}}} \mathbf{( k m )}$ & $\overline{\mathbf{V p} / \mathbf{V s}}$ & $\boldsymbol{\sigma}_{\overline{\mathbf{k}}}$ \\
\hline TRVA - Grupo 1 & 34.8 & 0.1 & 1.73 & 0.004 \\
\hline TRVA - Grupo 2 & 34.6 & 0.2 & 1.65 & 0.06 \\
\hline USHA - Grupo 1 & 27.6 & 0.3 & 1.66 & 0.01 \\
\hline USHA - Grupo 2 & 27.8 & 0.1 & 1.80 & 0.005 \\
\hline DSPA - Grupo 1 & 24.8 & 0.1 & 1.63 & 0.01 \\
\hline DSPA - Grupo 2 & 30.1 & 0 & 1.56 & 0 \\
\hline ELCA - Grupo 1 & 28.6 & 0.05 & 1.77 & 0.02 \\
\hline
\end{tabular}

Tabla 3.1: Valores medios y su desviación estándar del resultado de H y k obtenido en cada grupo y para cada estación. 


\section{Inversión no-lineal de Funciones del Receptor: Método Neighbourhood Algorithm}

\subsection{Introducción}

Un método eficaz para estimar la estructura cortical en una región es el de la inversión de FRs (Owens et al., 1987; Ammon et al., 1990; Sambridge et al., 1996; Shibutani et al., 1996; Piana Agostinetti et al., 2002; Zor et al., 2003; Frederiksen et al., 2003; Lawrence y Wiens, 2004; Gosselin et al., 2015). Mediante esta técnica, las FRs son invertidas a un modelo unidimensional de velocidades de onda $\mathrm{S}$ que reproduce una estimación de la estructura sísmica debajo de una estación sismológica. Sin embargo, el problema de inversión de FRs es complejo por su nolinealidad y por la no-unicidad de sus soluciones (Ammon et al., 1990; Sambridge, 1999). Resolver la inversión mediante el cálculo de derivadas parciales respecto de los parámetros del modelo es complicado de realizar, además las soluciones suelen quedar atrapadas en mínimos locales y son dependientes de los modelos iniciales.

Una estrategia muy utilizada para resolver los problemas de inversión es la aplicación de los métodos de búsqueda directa. Es decir, resolver el problema directo iterativamente, estimando los parámetros del modelo, prediciendo los datos y evaluando el error entre los datos observados y los calculados. Los primeros métodos de búsqueda directa se basaban en un muestreo uniforme pseudo-aleatorio del espacio de los parámetros (Wiggins, 1969). De esta manera, los métodos de búsqueda de Monte Carlo comenzaron a implementarse como una alternativa en los problemas de inversión de FRs. Dichos métodos consisten en realizar una búsqueda aleatoria para acelerar el muestreo uniforme del espacio del modelo. Estos incluyen técnicas de optimización global como Genetic Algorithm (GA) (Shibutani et al., 1996; Levin y Park, 1997; Clitheroe et al., 2000; Chang et al., 2004), Niching Genetic Algorithm (NGA) (Lawrence y Wiens, 2004), Simulated Annealing (SA) (Vinnik et al., 2004b, 2006), Very Fast Simulated Annealing (Zhao et al., 1996). Los métodos de búsqueda estocásticos como el método Uniforme de Monte Carlo (UMC), SA y GA resultaron muy populares al momento de encontrar familias de modelos ubicados en un espacio de parámetros 
multidimensional que minimizan la diferencia entre las predicciones teóricas y las observaciones. La función que mide el ajuste de las observaciones por las simulaciones teóricas se llama función objetivo o de desajuste (misfit function). En este trabajo se utiliza el término función de desajuste y el objetivo es minimizar dicha función. Todas las metodologías mencionadas anteriormente se diferencian entre sí en la estrategia de muestreo del espacio multidimensional de los modelos, sin estancamientos de modelos en mínimos locales.

Posteriormente Sambridge (1999) desarrolló el método denominado Neighbourhood Algorithm (NA). La propuesta se basó en un nuevo algoritmo de optimización en el que la búsqueda de nuevos modelos es mejor guiada si se tiene en cuenta la información de modelos previos en los que la búsqueda directa fue resuelta. Por definición el método UMC no utiliza información de modelos existentes ya que cada muestra nueva es independiente de las muestras previas. En el caso de los métodos SA y GA, sí se utiliza información de modelos obtenidos previamente pero de manera diferente. Un método de búsqueda aleatorio, como es el de Monte Carlo, puede dar como resultado el mejor modelo pero requiere un mayor costo computacional, por no ser un método que guía el muestreo en el espacio de los modelos, un factor que se tiene en cuenta cuando se trabaja con gran cantidad de datos.

El método utilizado en este trabajo es el NA (Sambridge, 1999). Este método es similar a los métodos GA y SA y constituye una buena alternativa para resolver problemas de inversión nolineales. A través de cada iteración se realiza un ajuste del modelo inicial para sucesivas capas con aumentos o descensos graduales de la velocidad. Es decir, el método consiste en minimizar las diferencias entre las FRs observadas y las calculadas a partir de ciertos modelos de prueba. Además, el NA utiliza la información de las muestras del espacio de modelos previos y aproxima la función de desajuste en todos los sitios del espacio del modelo. Cuando se aplica este método es necesario asumir un modelo inicial de velocidades unidimensional e isótropo y el problema de inversión es resuelto mediante una serie de iteraciones sin requerir el cálculo de derivadas parciales. La solución no es única y para poder restringir las posibles soluciones es necesario disponer de cierta información previa de la zona de estudio. A continuación se brindan más detalles de este método.

\subsection{Método Neighbourhood Algorithm (NA)}

Sambridge (1999) propuso un algoritmo que realiza una optimización en los problemas de inversión. El método consiste en realizar un muestreo del espacio de los modelos, seleccionando las regiones con mejor ajuste entre las observaciones y los modelos, volviéndolas a muestrear de manera que las regiones más muestreadas sean aquellas donde se obtiene el mejor ajuste de los 
modelos con los datos. Este método pertenece a la familia de los métodos de optimización de Monte Carlo y se basa en lo siguiente:

"La filosofía detrás de este algoritmo es que el ajuste de cada modelo previo es representativo de la región de su espacio en su vecindario”. (Sambridge, 1999, p484).

El método NA utiliza un concepto geométrico definiendo las celdas de Voronoi que son las regiones más cercanas alrededor de las muestras del modelo cuyo punto medio coincide con la muestra del modelo. La clave de la técnica es generar nuevas muestras mediante un remuestreo de las celdas de Voronoi con densidad local uniforme. En la siguiente sección 4.2.1 se describe con más detalle el concepto de estas celdas.

Este algoritmo depende sólo de dos parámetros de control: el número de modelos generados en cada iteración Ns y el número de celdas a muestrear Nr. Si se selecciona un valor de Ns/Nr cercano a 1 el algoritmo será explorativo, es decir que el algoritmo realiza la búsqueda en todo el espacio de los modelos sin interés en regiones locales. $\mathrm{Si} \mathrm{Ns} / \mathrm{Nr}$ es cercano a 0 , el algoritmo utiliza información local para seleccionar los mejores modelos de la vecindad (Sambridge, 1999).

El procedimiento del algoritmo NA se puede resumir en los siguientes pasos:

(1) Generar un conjunto inicial de modelos Ns uniformemente distribuidos en el espacio de los parámetros.

(2) Calcular la función de desajuste para los modelos Ns generados y determinar los modelos Nr con el desajuste (misfit) más bajo.

(3) Generar nuevos modelos Ns realizando una búsqueda uniforme y aleatoria a través de las celdas de Voronoi en las cuales están los $\mathrm{Nr}$ modelos elegidos con el desajuste más bajo.

(4) Ir al paso 2.

(5) El algoritmo se detiene cuando realiza las iteraciones deseadas.

De esta manera en cada iteración las muestras nuevas estarán concentradas en los alrededores donde existe el mejor ajuste entre los modelos y los datos.

\subsubsection{Celdas de Voronoi}

El método NA utiliza un concepto geométrico para realizar la búsqueda en el espacio de los parámetros del modelo y se basa en la definición de celdas de Voronoi. El espacio de los modelos es dividido en celdas que son las regiones más cercanas alrededor de las muestras del modelo, con 
la muestra del modelo elegida en el punto medio de la celda. La ventaja de utilizar estas celdas, consiste en que las muestras nuevas estarán concentradas en las cercanías donde se obtiene un mejor ajuste entre los modelos y las observaciones. Para estos nuevos modelos, se calculan las FRs sintéticas y también el desajuste entre las FRs sintéticas y las observadas. En la siguiente iteración, se construyen nuevamente las FRs sintéticas para un nuevo conjunto Ns de modelos. Estos nuevos modelos son seleccionados teniendo en cuenta los $\mathrm{Nr}$ modelos previos con el desajuste más bajo. Entonces, de manera aleatoria se realiza una búsqueda a través de las llamadas celdas de Voronoi, con el modelo anterior como punto inicial y se utiliza para seleccionar las muestras del modelo siguiente. De esta manera, se seleccionarán los modelos de la región con el desajuste más bajo en lugar de los que presentan valores de desajuste más alto y así el algoritmo convergerá el modelo óptimo.

Consideremos un número $\mathrm{n}_{\mathrm{p}}$ de muestras en el espacio de un modelo para el cual se quiere determinar la función de desajuste utilizando la construcción geométrica de celdas de Voronoi. Cada celda corresponde a la región vecina más cercana alrededor de la muestra previa. Utilizado la norma $\mathrm{L}_{2}$ (II), la distancia entre los modelos $\mathrm{m}_{\mathrm{a}} \mathrm{y} \mathrm{m}_{\mathrm{b}}$ está dada de la siguiente manera:

$$
\text { II } m_{a}-m_{b} I I=\left[\left(m_{a}-m_{b}\right)^{T} \cdot C_{M}^{-1}\left(m_{a}-m_{b}\right)\right]^{1 / 2}
$$

donde $C_{M}$ es una matriz que no-dimensionaliza el espacio de los parámetros, por ejemplo la matriz covarianza de un modelo a priori. El tamaño y forma de las celdas de Voronoi dependerá de la elección de $\mathrm{C}_{\mathrm{M}}$. Una elección simple sería una matriz diagonal cuyos elementos sean $1 / \mathrm{s}_{\mathrm{i}}{ }^{2}$. En este caso se puede reducir la matriz $\mathrm{C}_{\mathrm{M}}$ a la matriz identidad multiplicando cada parámetro del eje por un conjunto de factores de escala $\mathrm{s}_{\mathrm{i}}(\mathrm{i}=1, \ldots ., \mathrm{d})$.

Si se considera que $\mathrm{P}=\left(\mathrm{m}_{1}, \ldots . ., \mathrm{m}_{\mathrm{np}}\right)$ es un conjunto de puntos de un espacio con dimensión $\mathrm{d}$, siendo $2 \leq \mathrm{n}_{\mathrm{p}} \leq \infty \mathrm{y} \mathrm{m}_{\mathrm{i}} \neq \mathrm{m}_{\mathrm{j}}$ para $\mathrm{i} \neq \mathrm{j}$, se puede realizar una definición formal de las celdas de Voronoi:

$$
V\left(m_{i}\right)=\left\{x I I I x-m_{i} I I \leq I I x-m_{j} I I\right\} \quad \text { para } \mathrm{j} \neq \mathrm{i},\left(\mathrm{i}, \mathrm{j}=1, \ldots \mathrm{n}_{\mathrm{p}}\right)
$$

siendo $\mathrm{V}\left(\mathrm{m}_{\mathrm{i}}\right)$ la celda de Voronoi del punto $\mathrm{m}_{\mathrm{i}}, \mathrm{m}_{\mathrm{i}}$ los puntos del conjunto P y II la norma $\mathrm{L}_{2}$.

Como la función de desajuste resulta conocida para todas las muestras previas, la aproximación del algoritmo NA a la superficie de desajuste (superficie NA) es generada estableciendo el desajuste como una constante dentro de cada celda. Entonces para evaluar el desajuste de aproximación en cualquier punto sólo se necesita encontrar la muestra que esté más cercana al punto nuevo. 
Las celdas de Voronoi son únicas para cualquier distribución y densidad de muestras, y su tamaño es inversamente proporcional a la densidad de la muestra. Esto quiere decir que la superficie NA tendrá variaciones pequeñas de desajuste donde están presentes las muestras originales y, variaciones de gran escala donde el muestreo es escaso. Lo interesante de este concepto es que el tamaño y forma del vecindario alrededor de cada muestra está determinado completamente por la muestra en sí misma.

A continuación se muestra un ejemplo que explica gráficamente cómo se generan las celdas de Voronoi utilizando la información de las muestras previas: En la Figura 4.1 se observan 9 puntos negros, uno para cada celda de Voronoi, y 7 círculos en la zona sombreada de la celda del medio. Estos 7 círculos representan el centro de los siguientes modelos, es decir, se transforman en puntos iniciales para las siguientes muestras. A medida que el algoritmo realiza estos pasos, las celdas previas se van achicando a medida que se van generando nuevas celdas. Mientras el espacio de los parámetros se divide en pequeñas celdas de Voronoi, las muestras mantienen siempre la distancia con su vecindario y así el borde de la celda está en el camino medio entre el punto inicial y el de cada vecindario. Esto hace que cada celda sea única.

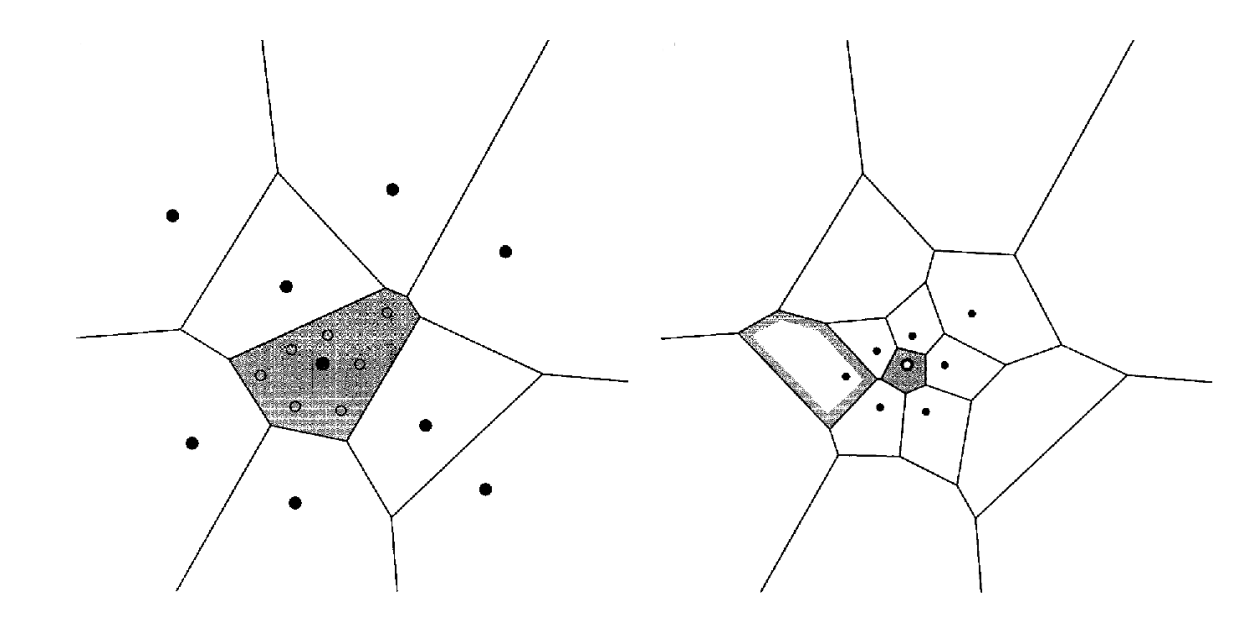

Figura 4.1: Ejemplo de cómo se construyen las celdas de Voronoi y cómo puede aumentar, disminuir o mover el centro de la densidad de muestreo en el algoritmo NA. A la izquierda, nueve puntos negros rellenos están distribuidos con sus celdas de Voronoi correspondientes. En la celda sombreada se agregan por ejemplo 7 puntos (círculos vacíos) y se disminuye la densidad de muestreo (imagen a la derecha). Si en la imagen de la derecha, el nuevo modelo sombreado que se muestra a la izquierda se convierte en el mejor ajuste entre los datos observados y calculados, entonces en la siguiente iteración los nuevos modelos se generarán en dicha celda. De esta manera la densidad del muestreo disminuirá y la región a muestrear se volverá a mover.

(Imagen extraída de Sambridge, 1999).

A continuación se presenta otro ejemplo de un problema de dos parámetros ilustrado en la Figura 4.2. En el panel superior izquierdo se observa el conjunto inicial de 10 puntos distribuidos 
cuasi-aleatoriamente. En cada etapa del NA, el espacio es dividido en un conjunto de celdas de Voronoi, cada una alrededor del modelo previamente muestreado. Estas celdas se utilizan para guiar el siguiente muestreo de manera aleatoria. A medida que las iteraciones aumentan, el algoritmo se concentra en las regiones donde el ajuste entre los modelos y los datos es mejor. En este ejemplo, el algoritmo NA distribuye 20 nuevos puntos en las mejores 20 celdas de Voronoi en cada iteración. El panel superior derecho muestra las celdas de Voronoi después de 100 puntos, y el panel inferior izquierdo muestra la geometría de la búsqueda luego de agregar 500 puntos más. Claramente todos los máximos son bien muestreados de esta manera. Sambridge (1999) demostró que a pesar que el NA se basa en un concepto geométrico, su aplicación resulta en una búsqueda altamente adaptativa y computacionalmente práctica hasta en problemas de espacios con grandes dimensiones (ver panel inferior de la derecha de la Figura 4.2).

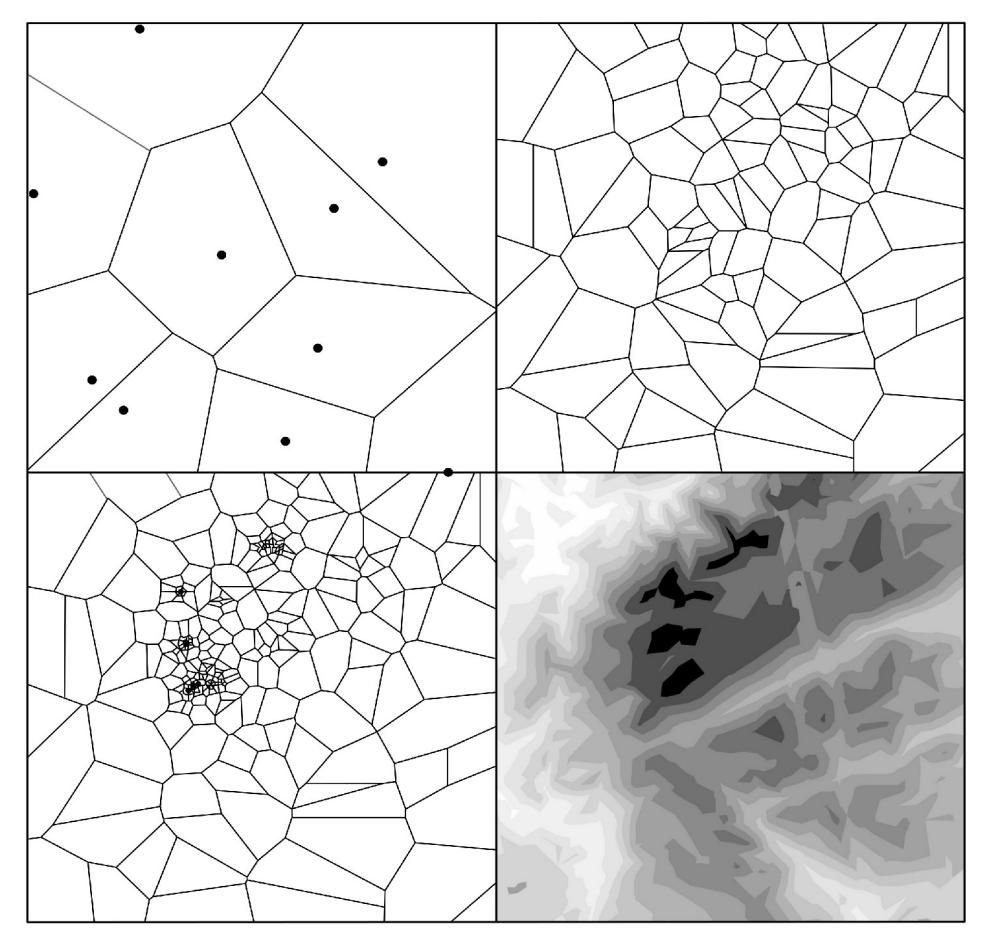

Figura 4.2: La figura ilustra las tres etapas del NA. El panel superior a la izquierda muestra 10 puntos iniciales distribuidos aleatoriamente con sus correspondientes celdas de Voronoi. El panel superior a la derecha muestra las celdas de Voronoi para los 100 primeros puntos generados por el NA y el panel inferior a la izquierda muestra las celdas de Voronoi para 500 puntos. En el panel inferior a la derecha se observan zonas oscuras que corresponden a aquellas para las cuales se obtuvo el mejor ajuste. (Extraído de Sambridge, 1999).

\subsubsection{Ventajas del NA}

Se pueden enunciar tres ventajas principales si se compara el método NA con los métodos SA y 
GA:

- Sólo se involucran dos parámetros del modelo: el número de muestras del modelo en cada iteración Ns y el número de modelos remuestreados Nr. Además del número de iteraciones que hay que definir.

- La información de modelos previos es utilizada de una manera más práctica con el fin de minimizar el tiempo de cálculo computacional.

- La utilización de celdas de Voronoi favorece a que las soluciones no queden atrapadas en mínimos locales, de esta manera se realiza un mejor muestreo del espacio de los modelos y se obtiene una convergencia más rápida.

El método NA utiliza las muestras previas para aproximar la función de desajuste en todos los lugares del espacio del modelo de manera contraria al UMC en el que cada muestra nueva es independiente de la muestra previa. Esta aproximación propuesta por Sambridge (1999) se puede incorporar a cualquier método de búsqueda directa. Por ejemplo se podría utilizar el método GA o SA en el punto (2) del procedimiento del algoritmo (sección 4.2). Sin embargo el método propuesto por Sambridge (1999) utiliza las propiedades de las celdas de Voronoi para guiar el muestreo en el espacio de los parámetros del modelo, es decir, generando nuevas muestras o modelos mediante un remuestreo de las celdas de Voronoi con densidad local uniforme.

\subsection{Inversión no-lineal de FRs mediante el algoritmo NA en TdF}

\subsubsection{Definición del modelo inicial}

Para realizar la inversión de las FRs en este trabajo mediante el algoritmo NA, es necesario en primer lugar definir un modelo de velocidades sísmicas inicial. En la Tabla 4.1 se muestra la parametrización del espacio de los modelos utilizada en el proceso de inversión realizado en este trabajo. La estructura cortical fue dividida en 5 capas horizontales siendo la última capa la de mayor velocidad, y el modelo considera 4 parámetros en cada capa: el espesor, la velocidad de onda $\mathrm{S}$ en la parte superior $\left(\mathrm{V}_{\mathrm{S} 1}\right)$ e inferior $\left(\mathrm{V}_{\mathrm{S} 2}\right)$ de cada capa y la relación de velocidades sísmicas $\left(\mathrm{V}_{\mathrm{P}} / \mathrm{V}_{\mathrm{S}}\right)$. Además, el algoritmo permite definir un rango de variación de estos parámetros en cada capa como se observa en la tabla. Por ejemplo en la primer capa, somo se observa en la Tabla 4.1, el espesor varía entre 0 y $5 \mathrm{~km}$, las velocidades $V_{\mathrm{S} 1}$ y $V_{\mathrm{S} 2}$ varía dentro del rango 1.75 a 3.20 y la relación $\mathrm{V}_{\mathrm{P}} / \mathrm{V}_{\mathrm{S}}$ toma valores que van desde 1.65 a 2.20. Esta parametrización fue adoptada luego de realizar varias pruebas preliminares con diferentes modelos iniciales en las que se fue ajustando el rango de los valores de cada parámetro a medida que los datos calculados y observados tenían 
mejor ajuste. De esta manera, la inversión se realiza considerando un gran conjunto de modelos iniciales cuyos parámetros varían según indica la Tabla 4.1.

\begin{tabular}{|c|c|c|c|}
\hline Espesor $(\mathbf{k m})$ & $\mathbf{V}_{\mathbf{s 1}}\left(\mathbf{k m ~ s}^{-1}\right)$ & $\mathbf{V}_{\mathbf{s} 2}\left(\mathbf{k m ~ s}^{-1}\right)$ & $\mathbf{V}_{\mathbf{p}} / \mathbf{V}_{\mathbf{s}}$ \\
\hline $0-5$ & $1.75-3.20$ & $1.75-3.20$ & $1.65-2.20$ \\
\hline $5-15$ & $3.20-4.00$ & $3.20-4.00$ & $1.65-1.85$ \\
\hline $5-20$ & $3.10-4.00$ & $3.10-4.00$ & $1.65-1.85$ \\
\hline $10-20$ & $3.60-4.50$ & $3.60-4.50$ & $1.65-1.85$ \\
\hline $10-30$ & $4.00-5.00$ & $4.00-5.00$ & $1.65-1.85$ \\
\hline
\end{tabular}

Tabla 4.1: Parametrización del espacio de los modelos iniciales utilizados en la inversión. La parametrización está compuesta por 20 parámetros (5 capas).

\subsubsection{Parámetros de control}

La implementación del algoritmo NA requiere la definición de dos parámetros de control como se mencionó en la sección 4.2. Luego de varias pruebas preliminares realizadas con diferentes parámetros de control Nr y Ns, se adoptó un rango de valores para los mismos. De esta manera, la inversión es llevada a cabo para todas las combinaciones posibles de dichos parámetros:

- Número de modelos generados en cada iteración Ns: 20, 40, 50

- Número de celdas a muestrear en cada iteración Nr: 2, 3, 4, 5, 6, 7, 8, 9, 10, 20

En cuanto al número de iteraciones, se consideró la misma estrategia probando diferentes valores pero finalmente se adoptó la cantidad de 200 iteraciones para cada inversión. Es decir, en cada una de las 200 iteraciones, el algoritmo explora la cantidad de modelos (Ns) y remuestrea el número de celdas de Voronoi (Nr) definidos anteriormente para el presente estudio.

\subsubsection{Selección de datos}

En la sección 3.3.1 del Capítulo III, las FRs seleccionadas fueron agrupadas en: grupo 1 (eventos provenientes del SO) y grupo 2 (eventos provenientes del N). Para maximizar la SNR, se realizó el apilamiento de las FRs seleccionadas en cada grupo de cada estación. Se utilizó la ventana de tiempo correspondiente a $5 \mathrm{~s}$ antes del arribo de la onda P directa hasta 30 s después del 
mismo. Cada forma de onda presente en la Figura 4.3 corresponde a un apilamiento de las FRs y para cada una se realizó la inversión teniendo en cuenta los parámetros de control mencionados anteriormente. De esta manera, considerando el apilamiento de las FRs, se realizaron dos inversiones por estación (una para cada grupo) con excepción de ELCA que contó con un solo grupo. Cabe destacar que en cada inversión se tuvieron en cuenta todas las posibles combinaciones de los dos parámetros de control mencionados en la sección 4.3.2.

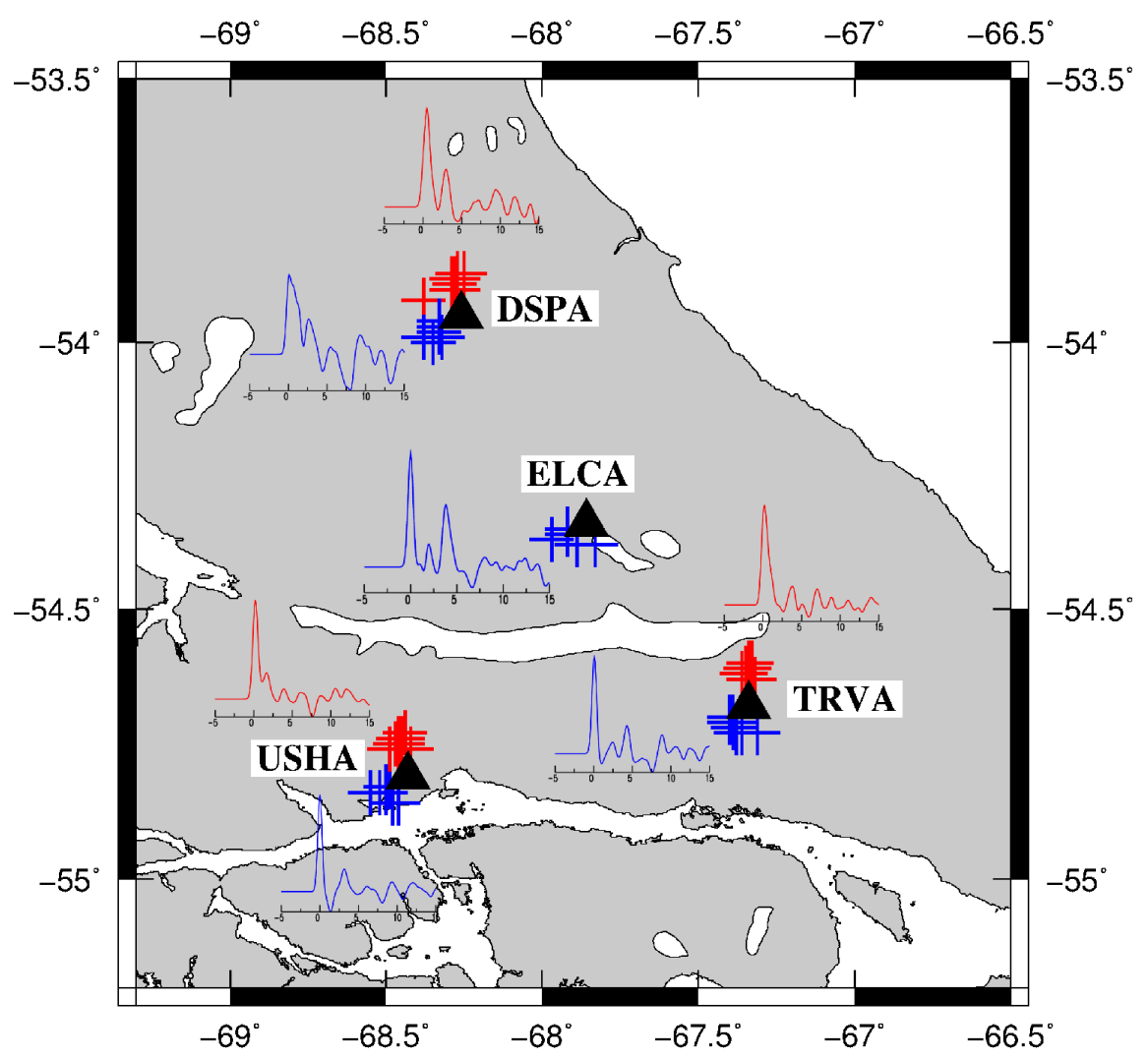

Figura 4.3: Apilamiento de las FRs radiales correspondientes al grupo 1 (eventos al SO de la estación, en azul) y grupo 2 (eventos al $\mathrm{N}$ de la estación, en rojo) para las cuatro estaciones sismológicas utilizadas en el proceso de inversión (en la Figura se muestra sólo los primeros 15 s de la forma de onda). Las cruces denotan

los puntos de conversión en profundidad de los eventos seleccionados considerando una profundidad hipotética de $30 \mathrm{~km}$. Nótese la diferente forma de onda de las FRs correspondientes a ambos grupos de las estaciones DSPA, TRVA y USHA.

\subsubsection{Resultados}

\subsubsection{Estación TRVA}

En las Figuras 4.4 y 4.5 se observa el resultado de aplicar el algoritmo NA a los grupos 1 y 2 de la estación TRVA mostrados en la Figura 4.3, respectivamente. El espacio de los parámetros está 
indicado por las líneas negras, y la zona sombreada gris es donde el algoritmo muestrea todas las posibles combinaciones de los parámetros de control. El área verde indica la densidad de los mejores 1000 modelos, el color es logarítmicamente proporcional al número de modelos, como se muestra en la escala. El modelo de velocidades $V_{S}$ y relación $V_{\mathrm{P}} / V_{S}$ que mejor se ajusta a los datos observados se indica en color rojo. Para el grupo 1 (al SO de TRVA), el modelo con mejor ajuste se caracteriza por tener la Moho a los $39.2 \mathrm{~km}$, mientras que para el grupo 2 (ubicado al $\mathrm{N}$ de TRVA) se encontró a los 36.6 km.

a)

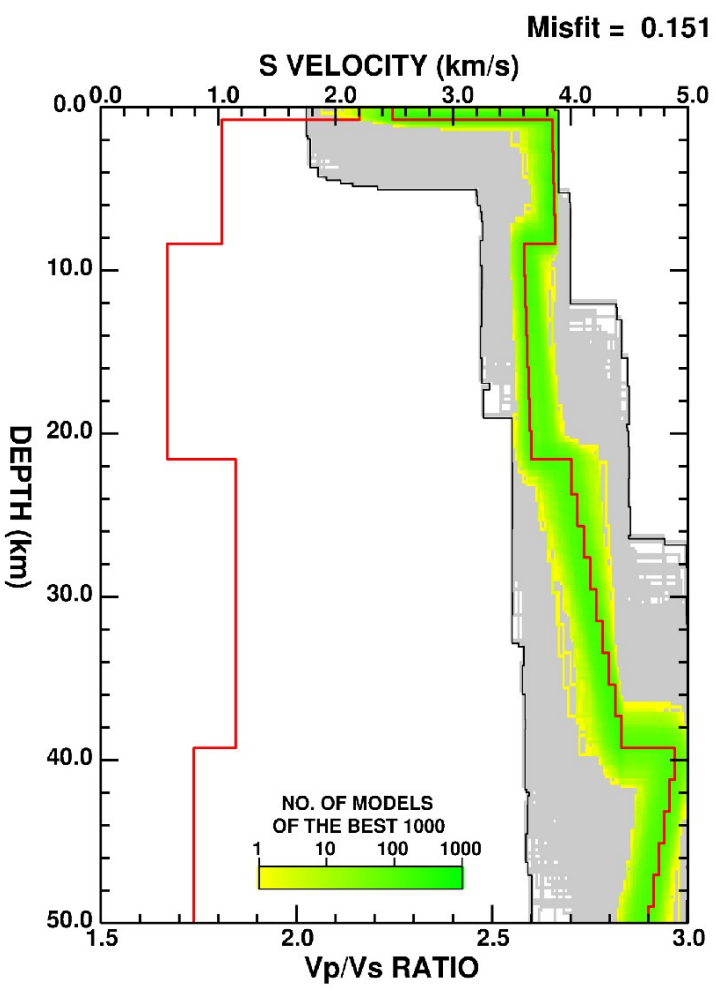

b)

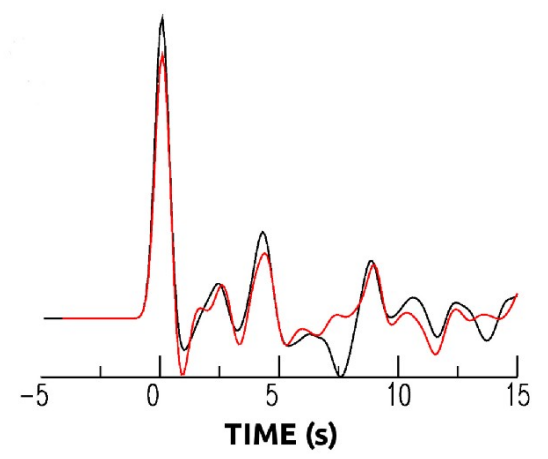

Figura 4.4: Resultado de aplicar el NA a las FRs radiales del grupo 1 para la estación TRVA (la ubicación de la estación se muestra en la Figura 1.2 del Capítulo I). a): modelo final (a la derecha) y relación $\mathrm{V}_{\mathrm{P}} / \mathrm{V}_{\mathrm{S}}$ que mejor ajusta con los datos observados (en rojo). b): comparación entre el apilamiento de las FRs radiales observadas (negro) y la FR radial sintética (rojo) correspondiente al mejor modelo obtenido. 


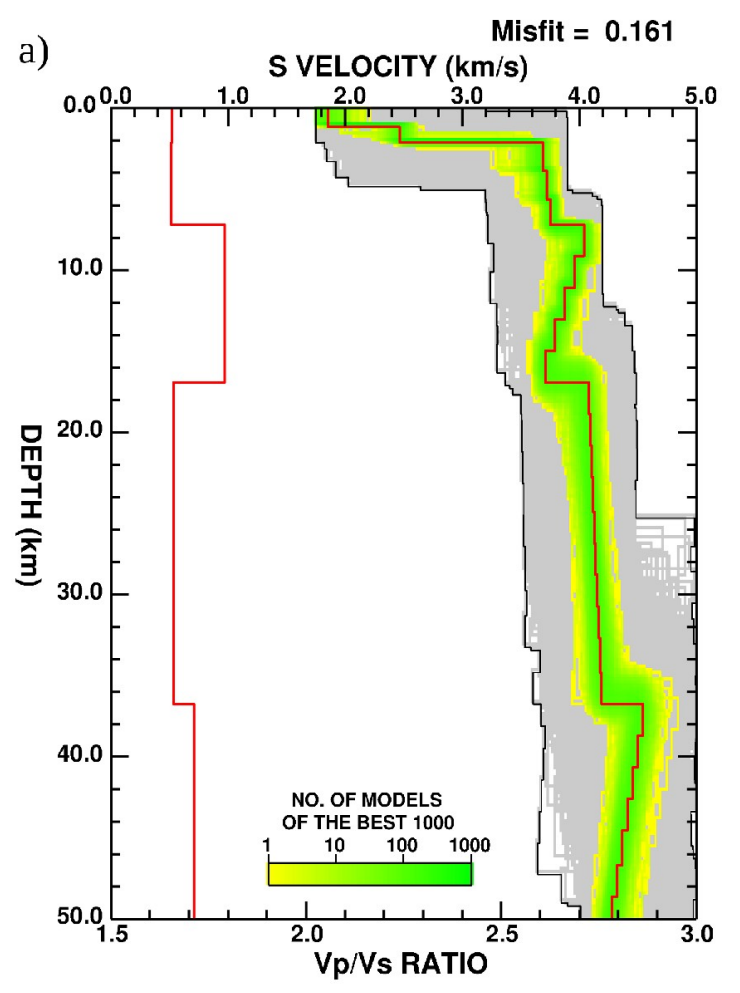

b)

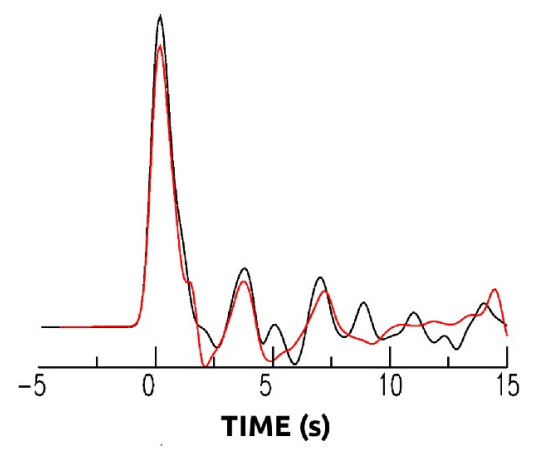

Figura 4.5: Resultado de aplicar la inversión NA a las FRs radiales del grupo 2 para la estación TRVA (la ubicación de la estación se muestra en la Figura 1.2 del Capítulo I). a): modelo final (a la derecha) y relación $\mathrm{V}_{\mathrm{P}} / \mathrm{V}_{\mathrm{S}}$ que mejor ajusta con los datos observados (en rojo). b): comparación entre el apilamiento de las FRs radiales observadas (negro) y la FR radial sintética (rojo) correspondiente al mejor modelo obtenido.

En ambos grupos se observa un rápido aumento de la velocidad $V_{\mathrm{S}}$ en las capas más superficiales. Asimismo se distingue una discontinuidad entre los 6 y $8 \mathrm{~km}$ de profundidad caracterizado por un aumento de velocidad para el grupo 2 y por una disminución de velocidad para el grupo 1. Dicha discontinuidad se observa a la misma profundidad en ambos grupos. En cuanto a la relación de velocidades sísmicas $\mathrm{V}_{\mathrm{P}} / \mathrm{V}_{\mathrm{S}}$, los valores promedios obtenidos mediante la técnica de NA son 1.87 (grupo 1) y 1.69 (grupo 2). La diferencia de estos valores encontrados puede deberse a que esta estación se encuentra en el límite de dos unidades geológicas distintas: Formación Lemaire y Formación Yaghan y Beauvoir, determinadas por Caminos y Nullo, (1979), 
Caminos et al., (1981), Olivero y Martinioni, (2001) y Ghiglione, (2003) (ver Figura 1.2 del Capítulo I). Además de las variaciones de composición de la geología, el grado de complejidad de la estructura en cuanto a geometría de fallas, discontinuidades o heterogeneidades volumétricas es diferente para cada grupo de esta estación lo cual se refleja en las diferentes formas de ondas obtenidas en las FRs.

\subsubsection{Estación USHA}

Los modelos que mejor ajuste tuvieron con los datos observados para la estación USHA corresponden a una estructura cortical con espesor de $32.3 \mathrm{~km}$ (grupo 1) y $34 \mathrm{~km}$ (grupo 2) y con el mismo valor de relación de velocidades sísmicas $\mathrm{V}_{\mathrm{P}} / \mathrm{V}_{\mathrm{S}}=1.81$ para ambos grupos.

a)

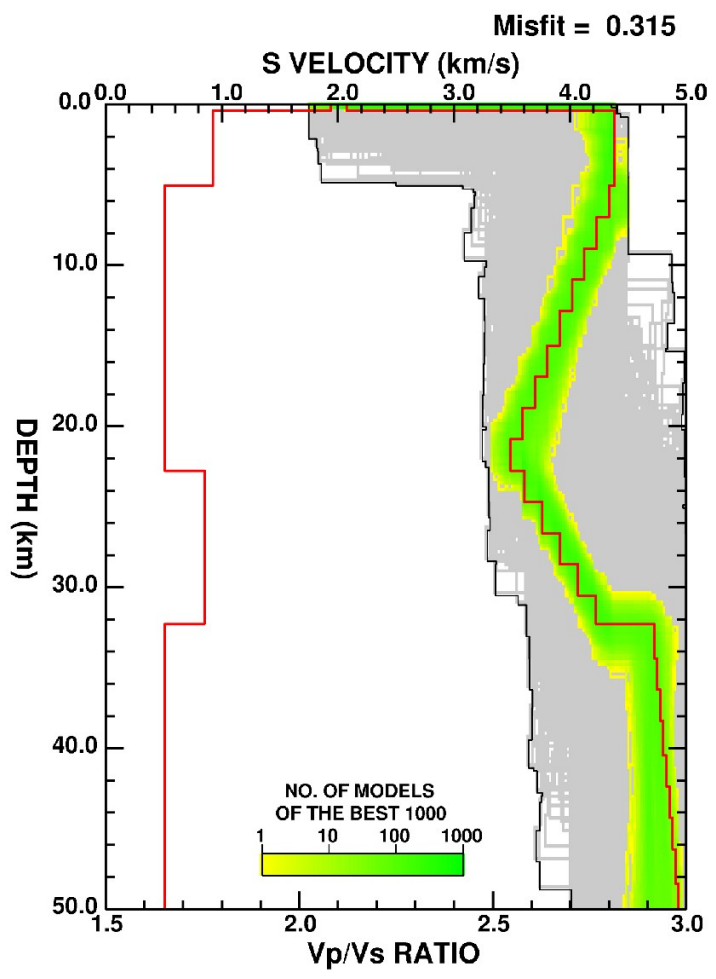

b)

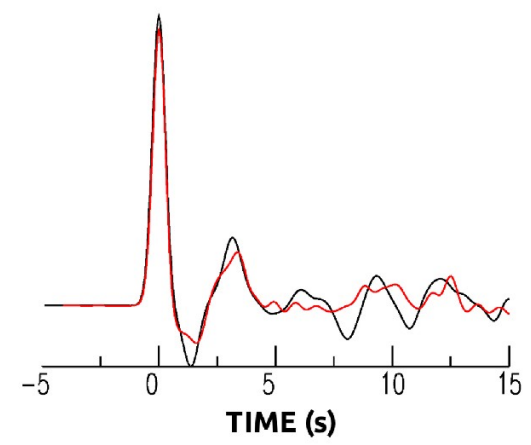

Figura 4.6: Resultado de aplicar la inversión NA a las FRs radiales del grupo 1 para la estación USHA (la 
ubicación de la estación se muestra en la Figura 1.2 del Capítulo I). a): modelo final (a la derecha) y relación $\mathrm{V}_{\mathrm{P}} / \mathrm{V}_{\mathrm{S}}$ que mejor ajusta con los datos observados (en rojo). b): comparación entre el apilamiento de las FRs radiales observadas (negro) y la FR radial sintética (rojo) correspondiente al mejor modelo obtenido.

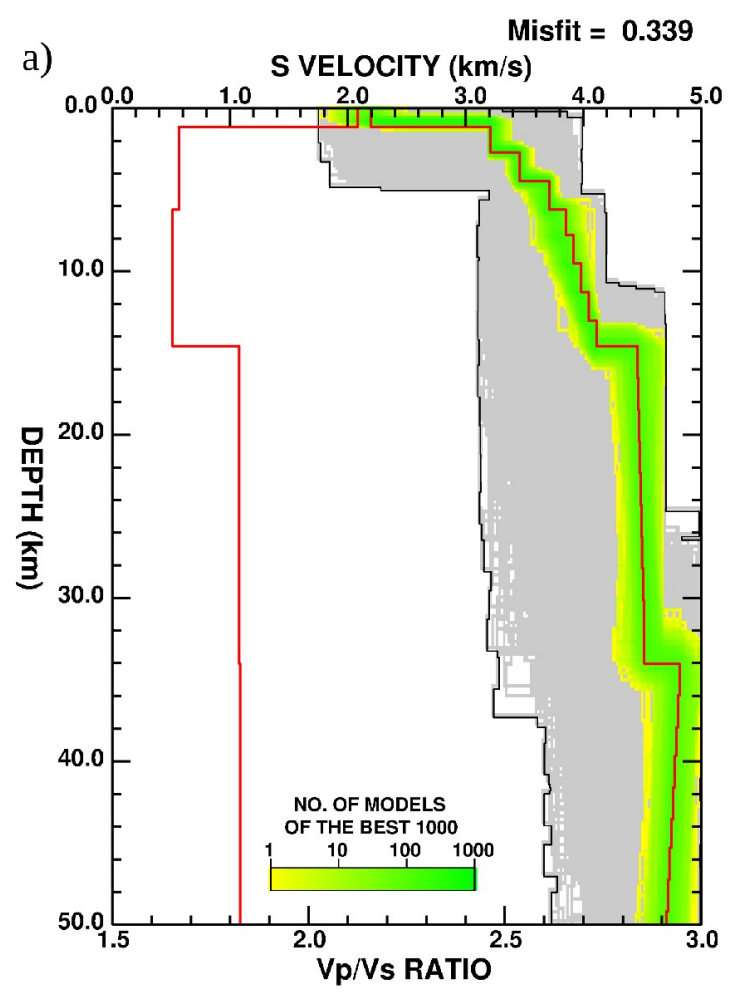

b)

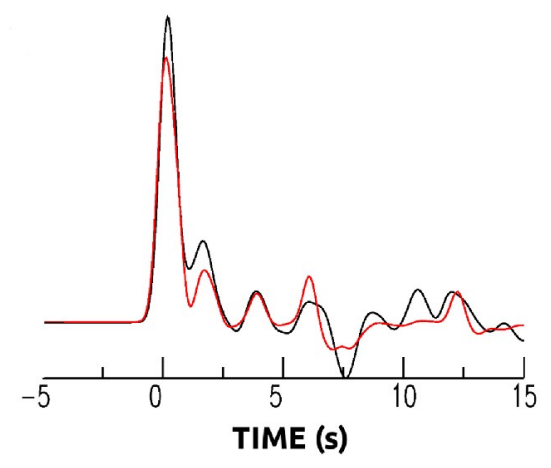

Figura 4.7: Resultado de aplicar la inversión NA a las FRs radiales del grupo 2 para la estación USHA (la ubicación de la estación se muestra en la Figura 1.2 del Capítulo I). a): modelo final (a la derecha) y relación $\mathrm{V}_{\mathrm{P}} / \mathrm{V}_{\mathrm{S}}$ que mejor ajusta con los datos observados (en rojo). b): comparación entre el apilamiento de las FRs radiales observadas (negro) y la FR radial sintética (rojo) correspondiente al mejor modelo obtenido.

En el modelo obtenido a partir de los eventos provenientes del SO a la región de estudio, se observa una disminución de la velocidad de onda $\mathrm{S}$ a partir de los $5 \mathrm{~km}$ evidenciado por la fase negativa que arriba antes de la conversión Ps (Figura 4.6b). Por otro lado, se observa una fase positiva antes de la conversión Ps (Figura 4.7b) indicando un incremento de velocidad para los 
datos del grupo 2. Este cambio de polaridad de la fase mencionada fue discutido en la sección 2.5.4.2 del Capítulo II, indicando la posible presencia de una capa con inclinación a los $5 \mathrm{~km}$ de profundidad. La presencia de dicha capa inclinada genera una disminución de velocidad en el modelo obtenido para el grupo 1 que es considerado como artilugio por no cumplirse la condición de capas horizontales, uno de los requisitos que se asume cuando se aplica el método de FRs.

\subsubsection{Estación DSPA}

Ambos grupos de la estación DSPA (mostrados en la Figura 4.3 de la sección 4.3.3), revelaron modelos finales con una Moho a los $26 \mathrm{~km}$ (grupo 1) y $24.5 \mathrm{~km}$ (grupo 2) de profundidad. Esto indicaría que el espesor cortical en la Isla aumentaría de norte a sur.

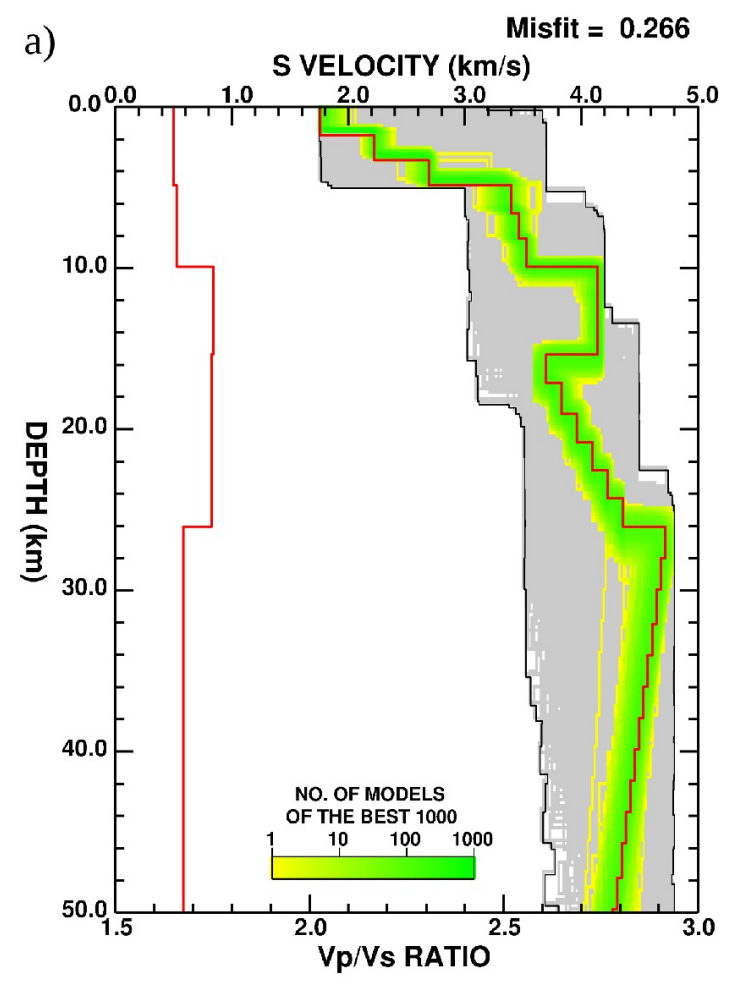

b)

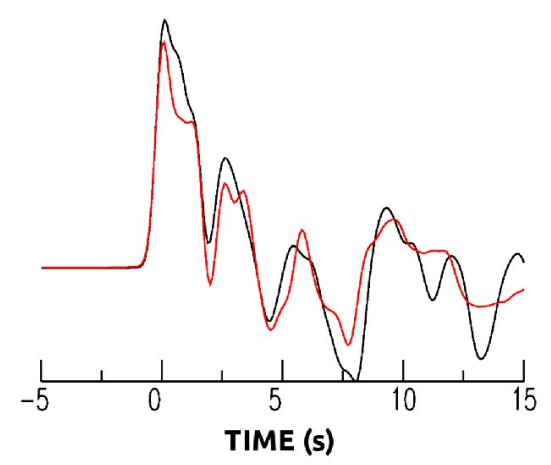

Figura 4.8: Resultado de aplicar la inversión NA a las FRs radiales del grupo 1 para la estación DSPA (la 
ubicación de la estación se muestra en la Figura 1.2 del Capítulo I). a) modelo final (a la derecha) y relación $\mathrm{V}_{\mathrm{P}} / \mathrm{V}_{\mathrm{S}}$ que mejor ajuste tiene con los datos observados (en rojo). b) comparación entre el apilamiento de las FRs radiales observadas (negro) y la FR radial sintética (rojo) correspondiente al mejor modelo obtenido.

Respecto a la relación de velocidades sísmicas, el mayor valor promedio encontrado en TdF (1.88) fue para el grupo 2 de la estación DSPA. La estructura de velocidad de onda S en esta estación, indica un considerable aumento entre los 4 y 8 km de profundidad (Figuras 4.8 y 4.9). A pesar de que en la estación DSPA se tuvieron que descartar un gran número de FRs por su mala calidad, fue posible con las FRs restantes obtener valores de $\mathrm{H}$ y $\mathrm{V}_{\mathrm{p}} / \mathrm{V}_{\mathrm{S}}$ que caracterizan la corteza de esa zona.

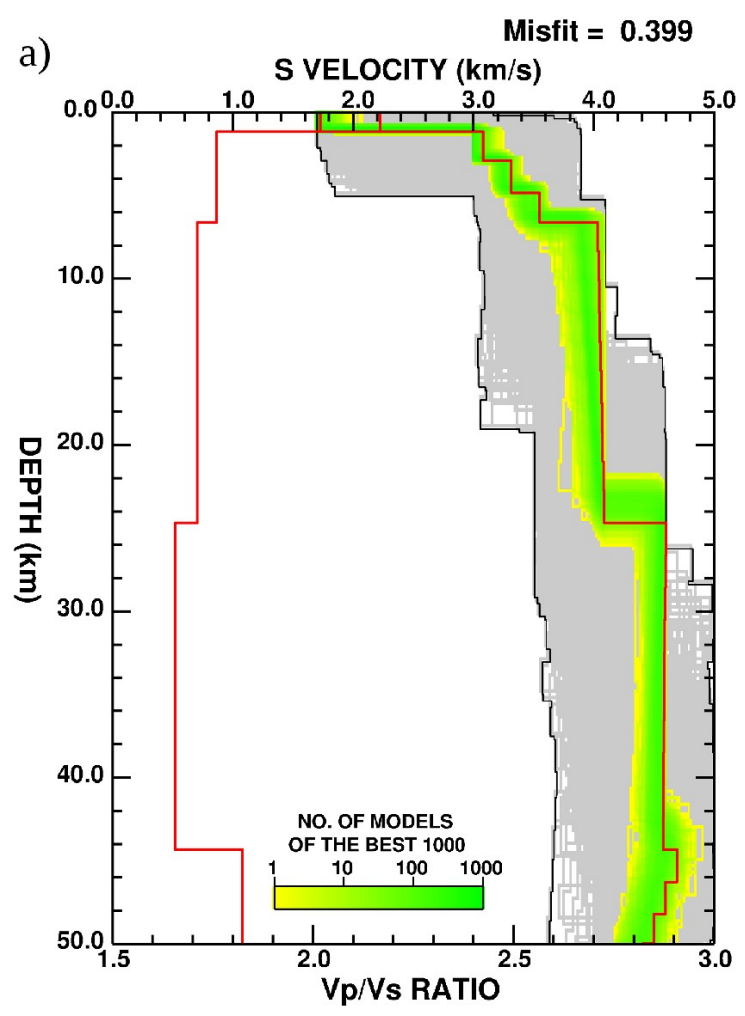

b)

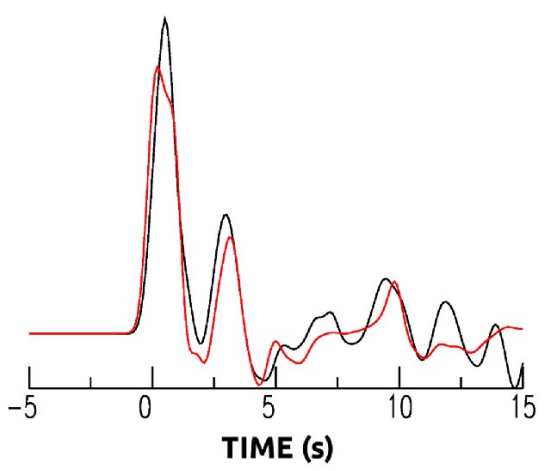

Figura 4.9: Resultado de aplicar la inversión NA a las FRs radiales del grupo 2 para la estación DSPA (la 
ubicación de la estación se muestra en la Figura 1.2 del Capítulo I). a) modelo final (a la derecha) y relación $\mathrm{V}_{\mathrm{P}} / \mathrm{V}_{\mathrm{S}}$ que mejor ajuste tiene con los datos observados (en rojo). b) comparación entre el apilamiento de las FRs radiales observadas (negro) y la FR radial sintética (rojo) correspondiente al mejor modelo obtenido.

\subsubsection{Estación ELCA}

Debido al número reducido de FRs seleccionadas en esta estación, el análisis se realizó sólo para los eventos provenientes del SO (grupo 1, mostrado en la Figura 4.3 de la sección 4.3.3). La estructura de velocidad de onda $\mathrm{S}$ revela un espesor cortical de $26.4 \mathrm{~km}$ indicando nuevamente el aumento gradual de espesor de norte a sur en la Isla (Figura 4.10).

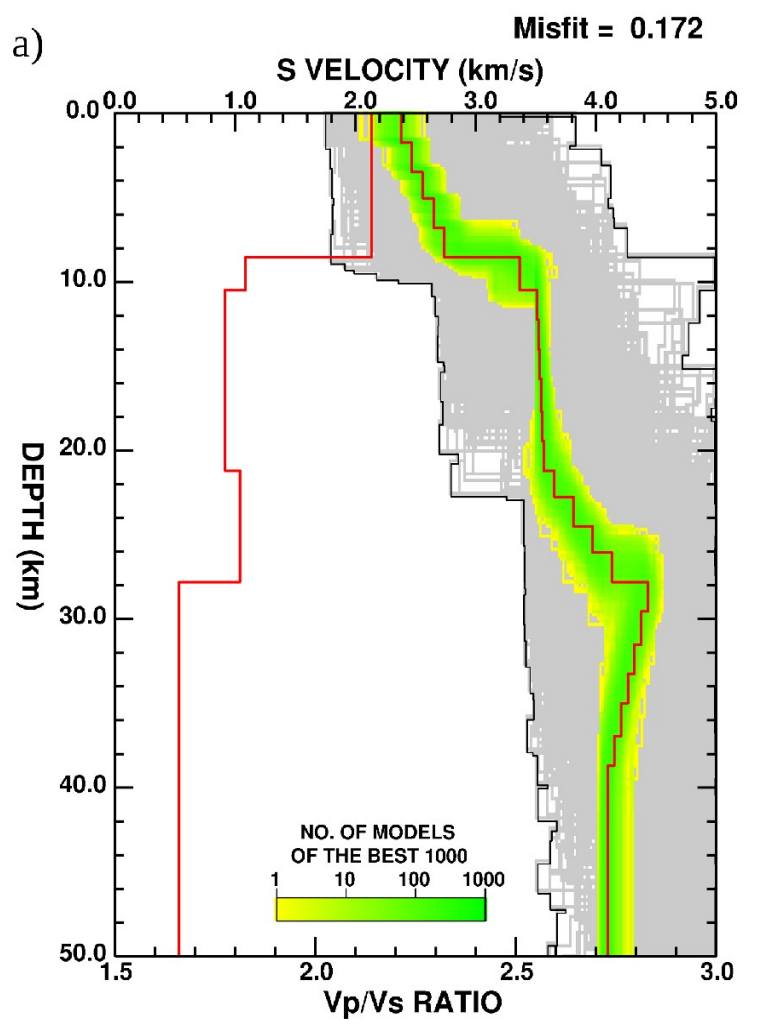

b)

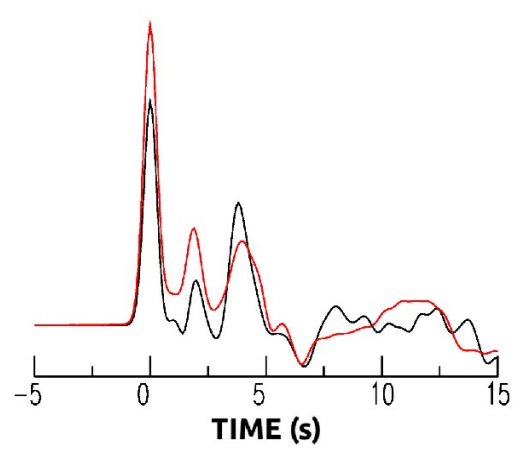

Figura 4.10: Resultado de aplicar la inversión NA a las FRs radiales del grupo 1 para la estación ELCA (la 
ubicación de la estación se muestra en la Figura 1.2 del Capítulo I). a) modelo final (a la derecha) y relación $\mathrm{V}_{\mathrm{P}} / \mathrm{V}_{\mathrm{S}}$ que mejor ajuste tiene con los datos observados (en rojo). b) comparación entre el apilamiento de las FRs radiales observadas (negro) y la FR radial sintética (rojo) correspondiente al mejor modelo obtenido.

La relación de velocidades sísmicas $\mathrm{V}_{\mathrm{P}} / \mathrm{V}_{\mathrm{s}}$ promedio correspondiente a dicho modelo es de 1.87. Se observa una pronunciada discontinuidad a los $8 \mathrm{~km}$ de profundidad. Además, el modelo obtenido en esta estación demuestra tener un rango de velocidad de onda $\mathrm{S}$ entre $2.4-2.7 \mathrm{~km} / \mathrm{s}$ en los primeros $8 \mathrm{~km}$ de corteza, a diferencia de los demás modelos finales que en su mayoría muestran un rápido incremento de $\mathrm{V}_{\mathrm{S}}$ con valores mayores a $3 \mathrm{~km} / \mathrm{s}$ en las capas más superficiales de la corteza.

\subsubsection{Inversión simultánea de FRs}

En lugar de invertir el apilamiento de las FRs de cada grupo, se puede buscar también el modelo que mejor ajusta a cada forma de onda que constituye el apilamiento, es decir, cada FR del grupo considerado. En este trabajo, el problema de inversión se resolvió de dos maneras: 1) realizando la inversión del apilamiento de las FRs y 2) realizando la inversión simultánea de las FRs sin considerar el apilamiento de las mismas. En ambos casos el resultado final es una familia de modelos que mejor ajusta a los datos observados, dentro de la cual se puede obtener un modelo óptimo. Los resultados de realizar la inversión considerando el apilamiento de las FRs fueron presentados en la sección 4.3.4.

En esta sección se presenta además, los resultados de aplicar el algoritmo NA considerando la inversión simultánea de las FRs, en cada grupo de cada estación. El hecho de que las estrategias de inversión mencionadas en los puntos 1) y 2) sean independientes, permite analizar si hay variaciones o similitudes entre los modelos finales ya que se espera modelos semejantes o iguales en un caso ideal.

Para realizar la inversión simultánea de las FRs, considerando el aporte de cada forma de onda individual, es necesario calcular el ángulo de incidencia de la primera onda P para cada FR. En un principio se puede asignar un peso a cada una de las FRs pero en este trabajo se consideró el mismo para todas. En cuanto al modelo de velocidades inicial y a los parámetros de control, se adoptaron los mismos valores utilizados en la inversión del apilamiento de las FRs.

En las Figuras 4.11 a 4.14 se observan los resultados de aplicar el algoritmo NA a cada grupo de cada estación, considerando las FRs individuales. El área verde indica la densidad de los mejores 1000 modelos, el color es logarítmicamente proporcional al número de modelos, como se muestra en la escala. El modelo que mejor ajusta a los datos observados se presenta en color azul. En el 
caso de la estación ELCA sólo se analiza la inversión del grupo 1.
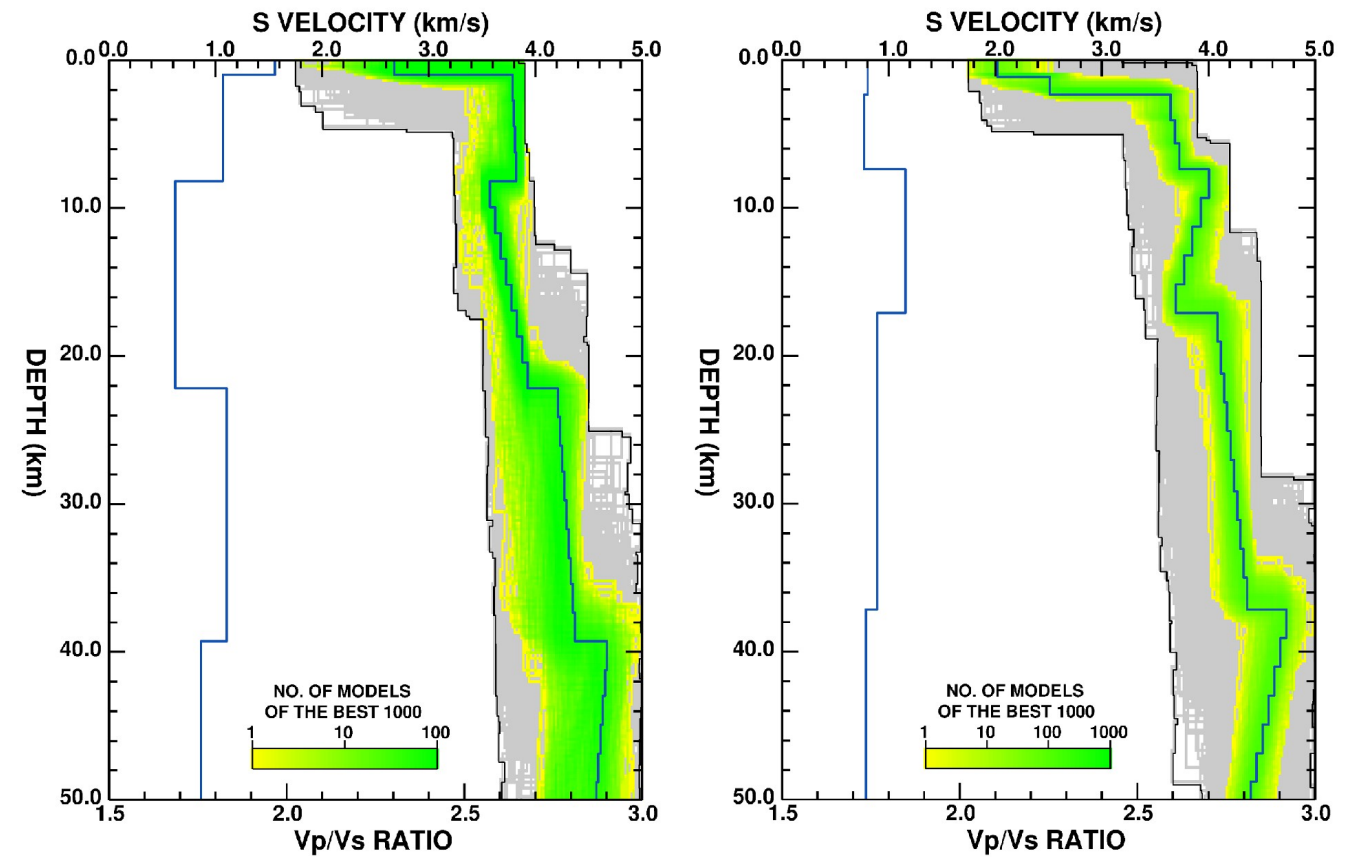

Figura 4.11: Resultado de la inversión simultánea considerando las FRs radiales del grupo 1 (izquierda, eventos del SO, Figura 4.3) y del grupo 2 (derecha, eventos del N, Figura 4.3) para la estación TRVA.
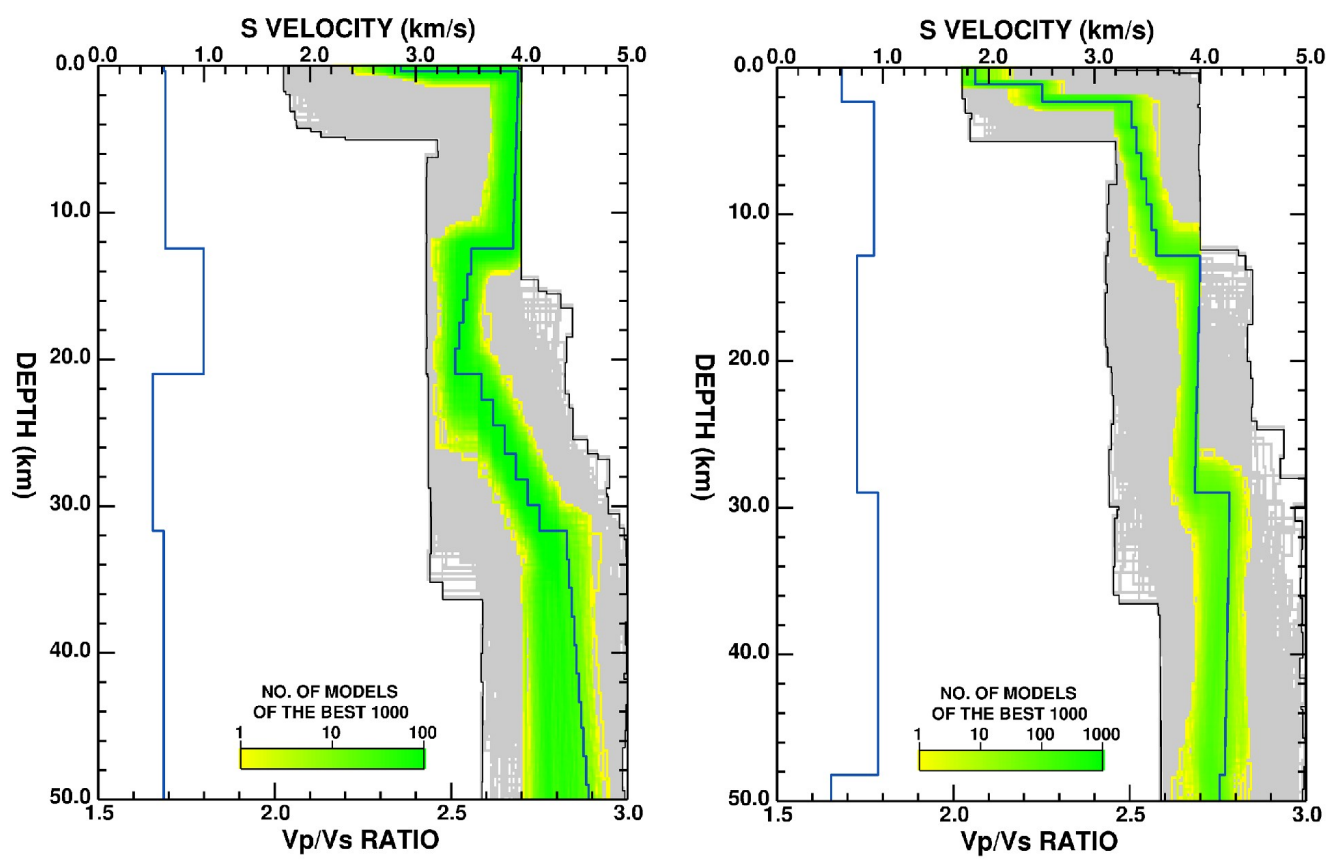

Figura 4.12: Resultado de la inversión simultánea considerando las FRs radiales del grupo 1 (izquierda, eventos del SO, Figura 4.3) y del grupo 2 (derecha, eventos del N, Figura 4.3) para la estación USHA. 

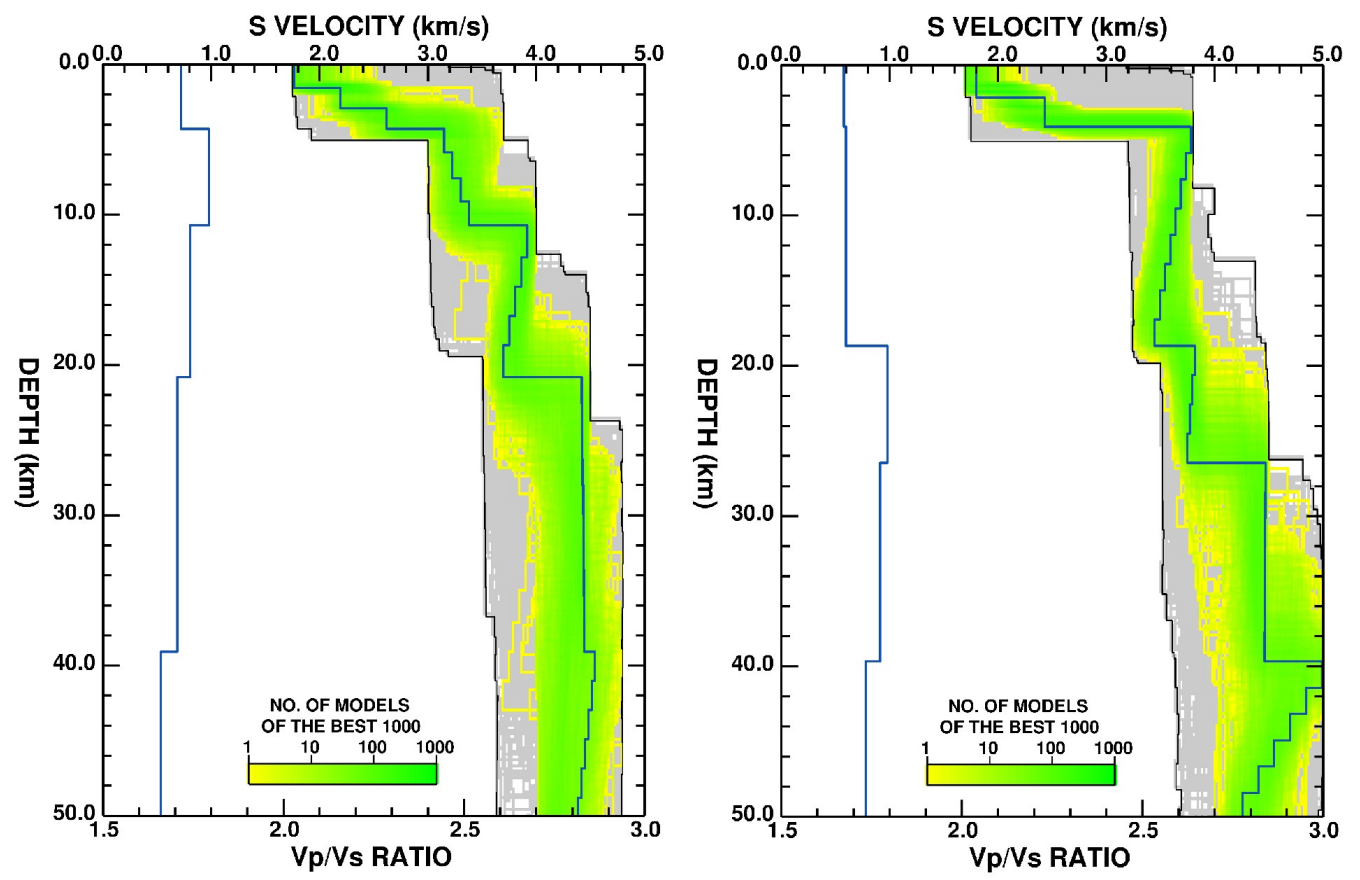

Figura 4.13: Resultado de la inversión simultánea considerando las FRs radiales del grupo 1 (izquierda, eventos del SO, Figua 4.3) y del grupo 2 (derecha, eventos del N, Figura 4.3) para la estación DSPA.

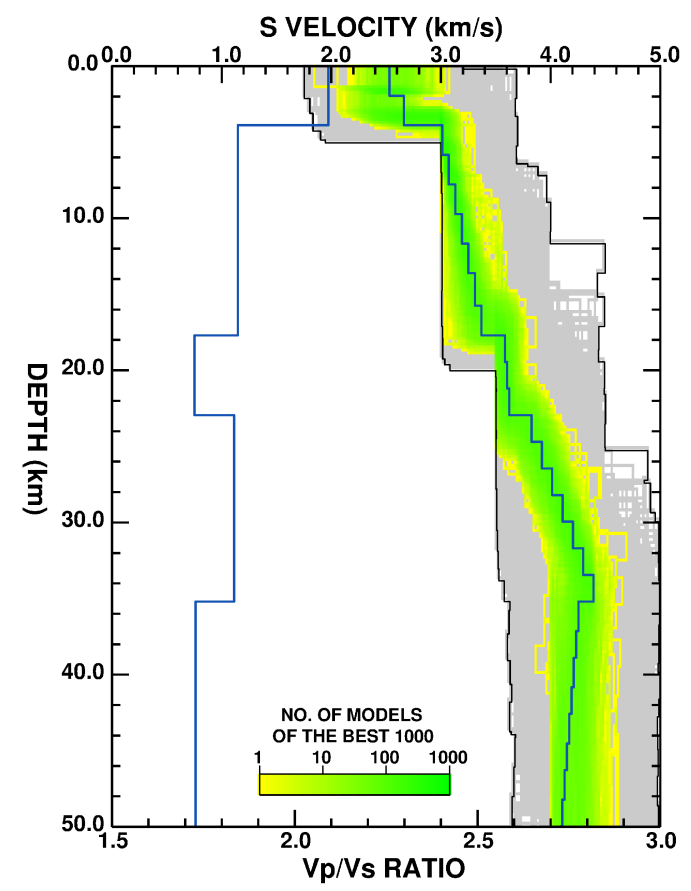

Figura 4.14: Resultado de la inversión simultánea considerando las FRs radiales del grupo 1 (eventos del SO, Figura 4.3) para la estación ELCA. 


\subsection{Discusión y conclusiones}

Los resultados obtenidos a partir de la inversión del apilamiento de FRs mediante el algoritmo NA, demuestran que la estructura cortical de TdF está caracterizada por un espesor que varía desde 24.5 a $39.2 \mathrm{~km}$. Estos valores se comparan favorablemente con estudios previos de refracción sísmica realizados en el Estrecho de Magallanes (a $150 \mathrm{~km}$ al oeste de la zona de estudio de este trabajo, aproximadamente) con resultados de 29.6 a $34.8 \mathrm{~km}$ de espesor (Ludwig et al., 1965), con los valores obtenidos a partir de la inversión regional de formas de onda (26-36 km) realizados en la Patagonia por Robertson Maurice et al. (2003), con los resultados obtenidos a partir de la inversión conjunta de FRs y velocidad de fase de ondas superficiales realizado en la Patagonia (28$34 \mathrm{~km}$ ) por Lawrence y Wiens (2004) y con los valores obtenidos mediante el método de H-k stacking desarrollado en el Capítulo III. Este último caso se discute en el Capítulo VII.

Los resultados de todas las inversiones con diferentes parámetros de control convergen a modelos similares. Los modelos obtenidos en las estaciones indican un aumento de espesor cortical hacia el sur de la Isla. Además, cuando se considera la inversión simultánea de las FRs, se observó una convergencia a modelos similares presentados para la inversión de las FRs apiladas, generando confianza en los resultados presentados.

Por otro lado, se pueden distinguir algunas características similares entre los modelos obtenidos en los diferentes grupos de cada estación:

Las velocidades $\mathrm{V}_{\mathrm{s}}$ más bajas ( 1.75-1.8 km/s) se obtuvieron bajo la estación DSPA en los primeros 1 a $2 \mathrm{~km}$ de profundidad. Dicho resultado se correlaciona con el ambiente geológico de la zona descripto por Olivero y Martinioni (2001) asociado con depósitos del Terciario. El modelo obtenido en la estación ELCA, demuestra que la estructura local en dicha estación está caracterizada por valores de $V_{\mathrm{S}}$ entre 2.4 y $2.7 \mathrm{~km} / \mathrm{s}$ en los primeros 8 $\mathrm{km}$ de corteza. Dicha estación se encuentra ubicada en la zona conocida como antepaís, donde se encuentran los mayores espesores sedimentarios cenozoicos (Olivero y Martinioni, 2001, Tassone et al., 2005; Menichetti et al., 2008).

> La localización del grupo 2 (eventos al N) de la estación TRVA y la del grupo 1 (eventos al SO) de la estación USHA coinciden según el ambiente geológico de la zona, como se observa en el mapa de la Figura 1.2 de la sección 1.3 del Capítulo I. Los modelos obtenidos en ambos grupos presentan similares valores de velocidad de onda $\mathrm{S}$ en el primer kilómetro de profundidad ( 1.9-2.1 km/s). Est resultado parece estar asociado con la presencia de depósitos del Cretácico Inferior (Tassone et al., 2005).

$>$ Las velocidades más altas en el primer kilómetro en profundidad de corteza ( $2.2-2.5$ $\mathrm{km} / \mathrm{s}$ ), se obtuvieron para el grupo 1 (eventos al SO) de la estación TRVA y el grupo 2 
(eventos al N) de la estación USHA. Ambos se ubican en la zona donde aflora la Formación Lemaire, compuesta por riolitas, basaltos, conglomerados, turbiditas, entre otros, del Jurásico Superior (Olivero y Martinioni, 2001)

> Además, en el caso de las estaciones ubicadas en el sur de la Isla (TRVA y USHA), la velocidad de onda S aumenta rápidamente en los primeros kilómetros de profundidad. Este incremento en las velocidades podría estar relacionado con algunos cuerpos magmáticos intrusivos presentes en la zona y que fueron detectados por otros métodos geofísicos (Tassone et al., 2005; Peroni et al., 2009). Por otro lado, Lawrence y Wiens (2004), determinaron $0 \mathrm{~km}$ de espesor de sedimentos en la estación VTDF (perteneciente al experimento SEPA y ubicada al sur de la Isla con coordenadas $54.14 \mathrm{~S}$ y $68.71 \mathrm{O}$ ) a partir de la inversión conjunta de FRs y velocidad de fase de ondas superficiales mediante la técnica NGA. Este estimación también parecería observarse en algunos de los modelos obtenidos donde se observa velocidades de onda S mayores en las primeras capas de la corteza.

$>$ La estructura de velocidad de onda S obtenida en la mayoría de las estaciones demuestra la presencia de una discontinuidad entre los 5 y $8 \mathrm{~km}$ de profundidad que se interpreta como el basamento al que hacen referencia Tassone et al. (2005) y Adaros Cárcamo (2003). Además, en los resultados obtenidos en este trabajo también se observa una profundización del basamento hacia el sur de la Isla como en el primer trabajo mencionado.

$>$ En algunos casos, los resultados indican un fuerte contraste positivo de velocidad entre los 15 y 17 km de profundidad. Dicha discontinuidad podría ser interpretada como el subbasamento observado en los perfiles de refracción sísmica realizados en el Estrecho de Magallanes por Ludwig et al. (1965).

En general todos los modelos obtenidos y las formas de ondas de las FRs seleccionadas demuestran que la estructura en esta zona es bastante compleja mediante la presencia de diversas fallas y estructuras geológicas. No obstante, fue posible la determinación de modelos corticales y la identificación de algunas discontinuidades intracorticales que se correlacionan con los resultados de estudios previos realizados mediante otras técnicas que exploraron niveles más superficiales en zonas cercanas al área de estudio. La inversión de las FRs fue llevada a cabo considerando el apilamiento y las formas de onda de las FRs individuales. En ambos casos, se realizaron diferentes pruebas y se analizaron los resultados considerando distintos parámetros de control. En todos los casos los resultados convergen a modelos similares demostrando la robustez de los resultados presentados. De todas maneras, la presencia de capas con inclinación en la estructura que no son modeladas correctamente por las FRs pueden generar un sesgado en los perfiles de velocidad. 


\section{Interferometría sísmica: Correlaciones cruzadas de ruido sísmico ambiental}

\subsection{Introducción}

Dentro de la Sismología, las herramientas o fuentes de energía más importantes para estudiar el interior de la Tierra son los terremotos y las explosiones. Desde hace más de un siglo que los terremotos están siendo registrados, localizados y utilizados para entender el interior terrestre. Las curvas de tiempo de recorrido de las ondas de cuerpo P y S, las velocidades de dispersión de grupo y fase de las ondas superficiales y la información derivada de los modos normales de la Tierra dieron información para determinar la estructura interna de la Tierra. De manera similar, la Sismología basada en fuentes explosivas, permitió estudiar la zona más superficial de la corteza con mayor resolución que con los terremotos. En ambos casos, el conocimiento de la estructura fue posible mediante la presencia de fuentes específicas (Bormann, 2002).

En las últimas dos décadas se introdujo el concepto de interferomería sísmica mediante el cual es posible estimar la estructura interna de la Tierra a través de un campo ondulatorio en un régimen difuso. Dentro de la interferometría sísmica se distingue la interfermetría de ruido (seismic noise interferometry) y la interferometría de coda (coda wave interferometry). Ambas se basan en los mismos principios y se distinguen básicamente por el origen del campo ondulatorio. Esta Tesis se centra en la interferometría de ruido sísmico ambiental ya que el mismo es ubicuo y abundante para el presente estudio. Esta técnica consiste en calcular la correlación cruzada del ruido sísmico registrado entre pares de estaciones sismológicas prescindiendo la ocurrencia de un terremoto. Así se comenzó a utilizar la información contenida en los registros de ruido sísmico para estimar la distribución de velocidades sísmicas del medio en el que se propagaron las ondas (Campillo y Paul, 2003; Shapiro y Campillo, 2004; Shapiro et al., 2005; Sabra et al., 2005b; Mottaghi et al., 2013).

Desde hace ya algunos años, se ha demostrado desde el marco teórico (Lobkis y Weaver 2001; Derode et al., 2003; Wapenaar, 2004; Snieder, 2004; Roux et al., 2005; Larose et al., 2005; Wapenaar et al., 2006; Snieder et al., 2007) y experimental (Campillo y Paul, 2003; Paul et al., 2005; Shapiro y Campillo 2004; Sabra et al., 2005a; Roux et al. 2005; Schimmel et al., 2011) que si 
se calcula la correlación cruzada entre dos sensores que registraron ruido ambiente, se puede obtener información de las propiedades del subsuelo.

El concepto de esta técnica se basa en la extracción de la Función de Green Empírica (FGE) entre dos sensores a partir de la correlación cruzada de registros continuos de ruido. Dicha función se detalla con más detalle en la sección 5.1.5.2. Dado que el ruido sísmico representa una fuente continua con un amplio contenido frecuencial, es posible a partir de este método, realizar estudios de estructura a diferentes escalas, monitorear una región ininterrumpidamente o detectar las fuentes principales de ruido, entre otros. En todos los casos, la resolución obtenida dependerá del número, la geometría y la distancia entre las estaciones sísmicas que se utilicen. En un caso ideal, se espera que la resolución sea mejor ya que cada estación actúa como una fuente virtual para la otra. Esto permite iluminar las heterogeneidades de manera más uniforme que con ondas generadas por terremotos, cuya ocurrencia espacial y temporal está limitada por ocurrir puntualmente y no en todas las regiones.

La interferometría sísmica es una técnica que está en constante evolución por ser una disciplina que recientemente despertó mucho interés. En este capítulo se pretende dar una visión simplificada con el objetivo de presentar el principio básico del método que se utiliza en este trabajo de tesis doctoral.

\subsubsection{Definición de ruido sísmico}

El ruido sísmico, también llamado ruido ambiente o ruido sísmico ambiental, es el registro temporal continuo de un sismómetro que no contiene sismos de naturaleza natural o artificial como pueden ser debido un terremoto o una explosión. (Figura 5.1)

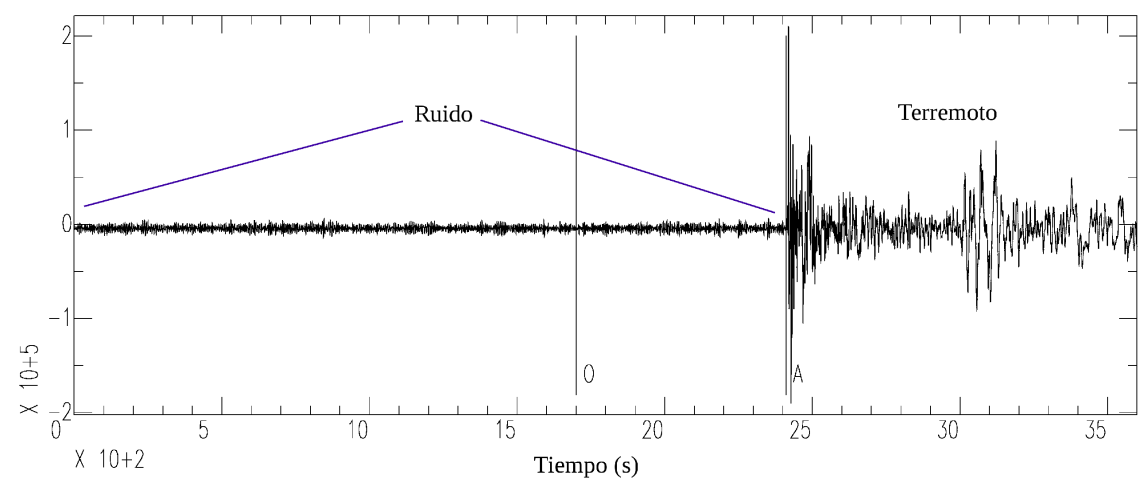

Figura 5.1: Registro de la componente vertical de una hora de duración obtenido en la estación TRVA donde se observa el comienzo de un terremoto y el ruido sísmico registrado previo a la ocurrencia del mismo. O y A indican la hora origen y el arribo de la onda P. Los datos del terremoto son: Fecha y hora origen: 09/12/2007, 07:28:20,82, latitud: $-26^{\circ}$, longitud: -177.51, profundidad: $152 \mathrm{~km}$, distancia epicentral: $79.9^{\circ}$ y magnitud: 
6.6. En este ejemplo, la señal previa al terremoto es considerada ruido y puede ser utilizada para estimar las propiedades del medio por el que se propagan las señales registradas.

El ruido sísmico solía ser una señal no deseada en los registros sísmicos que generalmente era removida para poder estudiar con detalle, por ejemplo, las características de un terremoto. Pero durante las últimas dos décadas el ruido sísmico es considerado una señal a partir de la cual se pueden obtener propiedades de la propagación de ondas superficiales e indirectamente de ondas de cuerpo entre dos receptores y donde uno de los receptores se puede considerar como fuente virtual.

\subsubsection{Tipos de ruido}

El ruido sísmico puede ser clasificado como natural o artificial dependiendo del origen del mismo. El clima, los océanos y la litosfera interaccionan entre sí dando como resultado la generación de ruido ambiente. Por ejemplo, una fuerte tormenta genera una perturbación en la atmósfera que impacta en el océano generando un patrón de ondas que crea una continua presión en el fondo del océano con intensidad variable. Esa perturbación en el fondo del océano genera ondas elásticas que viajan a través del medio y son registradas en los sensores como en el caso de un terremoto o una explosión. El mecanismo de generación de ondas sísmicas en aguas profundas y en áreas costeras es diferente como se explica más adelante. Lo mismo ocurre con las mareas terrestres, las olas, el viento, etc. En este caso se dice que el ruido sísmico es natural. La continua interacción de la baja frecuencia $(<1 \mathrm{~Hz})$ de las olas oceánicas con la corteza terrestre es la causa principal del ruido ambiente natural y este efecto es observado globalmente.

Por otro lado existe el ruido sísmico artificial o antropogénico, que es el generado por la actividad del hombre como por ejemplo debido a la industria, tráfico, maquinarias, actividades mineras y agrícolas, etc.

\subsubsection{Contenido espectral}

El ruido ambiente está compuesto por señales con diferente origen y contenido espectral (Figura 5.2). El espectro de frecuencias abarca un rango amplio que va desde las decenas de $\mathrm{Hz}$ hasta el orden de $10^{-3} \mathrm{~Hz}$.

Las diferentes fuentes naturales y artificiales que generan ruido sísmico en su mayoría no están relacionadas entre sí y son continuas. Además, forman parte de un proceso estacionario estocástico sin un espectro de fase definido por lo que la densidad espectral de amplitud y el espectro de fase 
no pueden ser calculados. En su lugar, se suele utilizar la densidad de potencia espectral (DPS) para caracterizar el contenido espectral de los registros. Para definir la DPS, es necesario recordar que la transformada de Fourier G de una función temporal g(t) es:

$$
G\left(f, T_{r}\right)=\int_{0}^{T_{r}} g(t) \mathrm{e}^{-i 2 \Pi f t} d t
$$

siendo $\mathrm{T}_{\mathrm{r}}$ la longitud de la serie temporal $\mathrm{y} f$ la frecuencia.

La densidad espectral de potencia $\mathrm{P}(\mathrm{f})$ se define como la transformada de Fourier de la autocorrelación de la señal g(t):

$$
P(f)=\int_{-\infty}^{\infty} a(\tau) \mathrm{e}^{-i 2 \Pi f \tau} d \tau
$$

donde a $(\tau)$ es la autocorrelación de la función temporal g(t).

Considerando valores discretos de frecuencia $\mathrm{f}_{\mathrm{k}}$, se puede escribir:

$$
G_{k}=\frac{G\left(f_{k}, T_{r}\right)}{\Delta t}
$$

con $f_{k}=\frac{k}{N \Delta t}$ para $\mathrm{k}=1,2, \ldots, \mathrm{N}-1, \quad N=\frac{T}{\Delta t}$ el número de muestras en cada segmento $\mathrm{T}$ de la serie y $\Delta \mathrm{t}$ el intervalo de muestreo.

Teniendo en cuenta las ecuaciones anteriores, la DPS se define como sigue:

$$
P_{k}=\frac{2 \Delta t}{N}\left(\left|G_{k}\right|\right)^{2}
$$

Este parámetro es muy utilizado para analizar el contenido espectral en registros que contienen ruido sísmico proveniente de diferentes tipos de fuentes (Figura 5.2).

El ruido de origen antropogénico genera señales de alta frecuencia con origen cercano al receptor. Por otro lado los eventos sísmicos como son los terremotos, abarcan un amplio rango del espectro que dependerá de la fuente del mismo, la estructura del medio y de la distancia que recorren las ondas sísmicas (desde 10 hasta $10^{-2} \mathrm{~Hz}$ ).

Un registro sísmico de ruido que no contenga señales debido a la actividad antropogénica o a la presencia de un terremoto, tendrá señales que dominan el espectro en el rango 0.05-0.4 Hz, 
conocido como "banda microsísmica”. La señal con menor frecuencia está relacionada con las oscilaciones libres de la Tierra y se denomina “hum”. Dicha señal está generada por vibraciones continuas del océano y la atmósfera en el rango de $10^{-2}-10^{-3} \mathrm{~Hz}$ (Nishida et al., 2009).
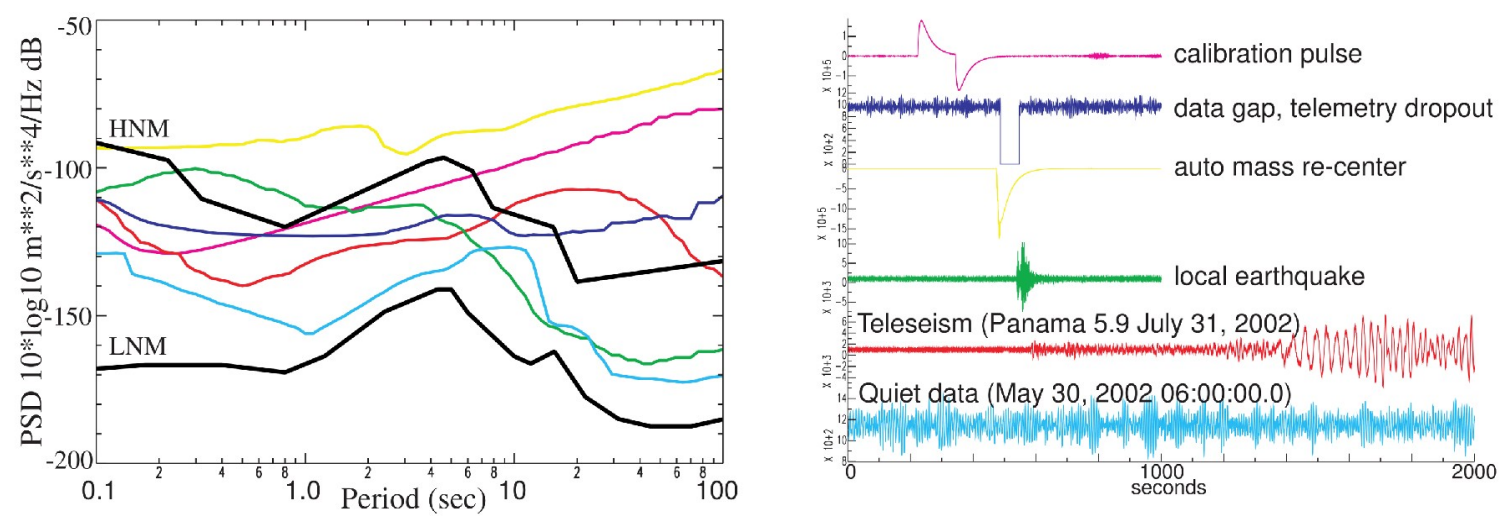

Figura 5.2: Ejemplo del contenido espectral de diferentes registros temporales mediante la DPS. Las líneas negras indican los modelos de ruido alto (HNM) y bajo (LNM) determinados por Peterson (1993). Se observa cada señal con su color y contenido espectral, respectivamente. (Imagen extraída de McNamara y Buland, 2004).

En la Tierra hay oscilaciones permanentes que incluyen muchas frecuencias. En la Tabla 5.1 se observa un rango típico de frecuencias generadas por diferentes fuentes sísmicas.

\begin{tabular}{|c|c|}
\hline Tipo de señal & Frecuencia (Hz) \\
\hline Mareas terrestres & $0.00001-0.0001$ \\
\hline Oscilaciones libres de la tierra, terremotos & $0.0001-0.001$ \\
\hline Ondas superficiales, terremotos & $0.001-0.01$ \\
\hline Ondas superficiales, ondas P y S, terremotos con M > 6 & $0.01-0.1$ \\
\hline Ondas P y S, terremotos con $\mathrm{M}>2$ & $0.1-10$ \\
\hline Ondas P y S, terremotos con $\mathrm{M}<2$ & $10-1000$ \\
\hline
\end{tabular}

Tabla 5.1: Esquema simplificado de frecuencias típicas generadas por fuentes sísmicas. M indinca la magnitud del terremoto.

El ruido sísmico de corto período puede tener un origen natural como el viento, mediante la fricción sobre el terreno, árboles u objetos que se balancean o vibran con el viento, etc. El espectro 
de este tipo de ruido comprende desde $0.5 \mathrm{~Hz}$ hasta 15-60 Hz (Young et al., 1996). Pero la fuente dominante del ruido sísmico de corto período es la actividad humana. La mayor parte del ruido sísmico abarca a las ondas superficiales por su generación en la superficie y por su menor atenuación. La penetración en profundidad de una onda superficial depende de la frecuencia y de la longitud de onda.

\subsubsection{Microsismo primario y secundario}

El término "microsismo" se suele utilizar para los registros de ondas sísmicas débiles y continuas que sólo puede ser detectado por sismógrafos. Los registros de este tipo están generados por ruido ambiente aunque también se utiliza este término para designar un sismo de pequeña magnitud $(\mathrm{M}<2.5)$. En esta Tesis el término microsismo refiere a aquellos que han sido generados por ruido sísmico.

El espectro del ruido sísmico está dominado por dos picos microsísmicos (primario y secundario) cuyos máximos se observan alrededor de los 15 s y 7 s de período, respectivamente (Figura 5.3). El mecanismo de generación de ambos microsismos está directamente vinculado con la actividad oceánica (Webb, 2007), con una gran influencia de la atmósfera mediante la ocurrencia de tormentas.

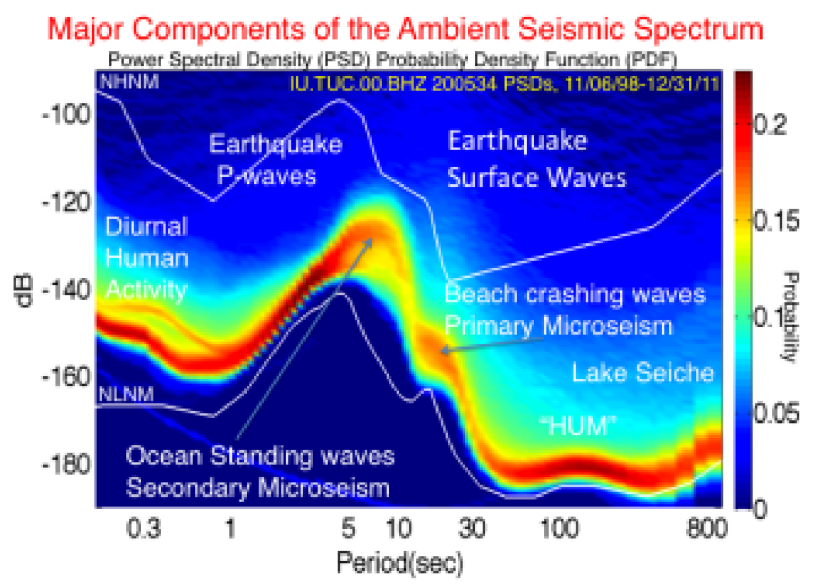

Figura 5.3: Ejemplo de los principales componentes presentes en el espectro del ruido ambiente. Los microsismos primarios (Primary Microseism) y secundarios (Secondary Microseism) están indicados con flechas. Se indica además la fuente principal de ruido en cada banda de frecuencia. NHNM y NLNM se definen en la Figura 5.2 como los niveles de ruido alto (HNM) y ruido bajo (LHN). (Extraído y modificado de McNamara and Buland, 2004). 
El microsismo primario se genera sólo en aguas someras en regiones costeras mediante la interacción de las olas oceánicas con el fondo marino. La energía de la onda es convertida en energía sísmica a través de las variaciones verticales de presión o a través de las olas cuando rompen en la playa (Figura 5.4) (Hasselmann, 1963 ; Haubrich et al., 1963).

Por otro lado, el mecanismo de generación del microsismo secundario está relacionado con la superposición de ondas del océano de igual período pero con dirección de propagación opuesta (Longuet-Higgins, 1950; Tanimoto, 2007). Las ondas generadas pueden interaccionar con las ondas reflejadas en la costa como se observa en la Figura 5.4. Las interacciones pueden ser con ondas generadas por una misma tormenta o incluso por diferentes tormentas separadas cientos a miles de kilómetros y días (Ardhuin et al., 2011). El principio de generación se basa en el mecanismo en el que las ondas de igual período pero con direcciones opuestas de propagación interfieren y generan perturbaciones que oscilan al doble de la frecuencia de la onda oceánica. Estas perturbaciones de presión pueden propagarse en aguas profundas y generar microsismos secundarios. La excitación más eficiente de ondas sísmicas se da para frecuencias a las cuales las perturbaciones de presión están en resonancia con el agua (Stutzmann et al. 2012; Gualtieri et al., 2013).

En ambos casos, el ancho, la amplitud y el período en el que prevalecen ambos microsismos dependerá de la localización del receptor respecto de la costa, de la latitud y de la época del año. Una característica de los microsismos oceánicos (primario y secundario) es que experimentan poca atenuación por lo que se pueden propagar cientos de kilómetros tierra dentro. Además el ruido microsísmico está presente en todo el globo terrestre.
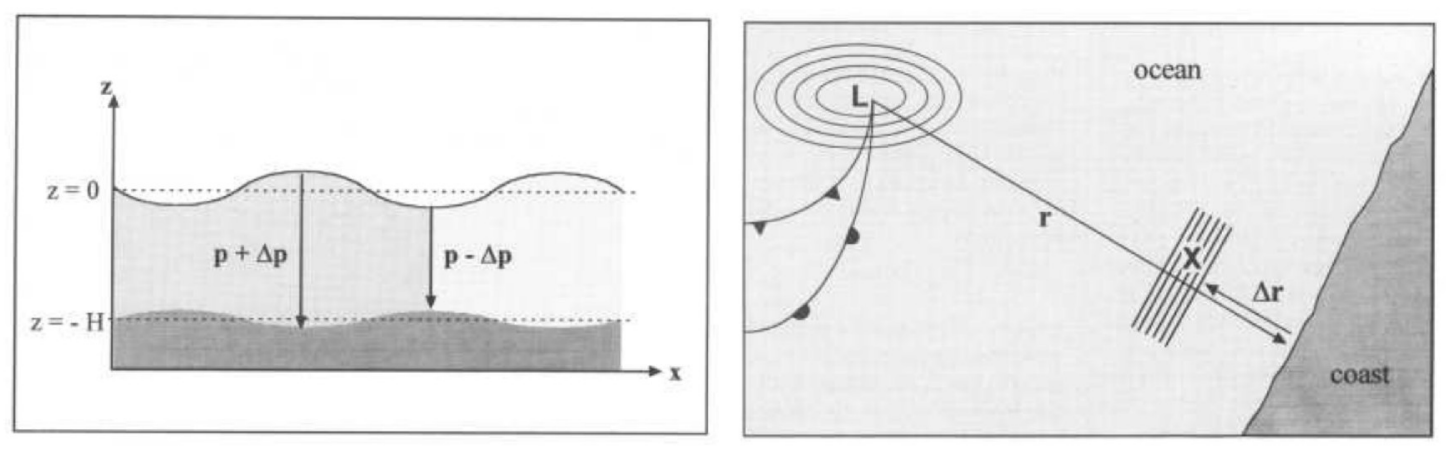

Figura 5.4: Esquema de los mecanismos de generación de microsismos primarios (izquierda) y secundarios (derecha). En el caso del microsismo secundario, "X" indica la zona de interacción de ondas con direcciones de propagación opuestas y L representa una zona de baja presión. (extraído de Friedrich et al., 1998). 


\subsubsection{Fundamentos}

\subsubsection{Correlación cruzada}

La correlación cruzada es una operación que mide la similitud de dos series temporales identificando el tiempo de retraso o "lag time" como se suele llamar en inglés, que se define como la cantidad de tiempo que una de las señales está desfasada respecto a la otra.

La correlación cruzada $\mathrm{C}(\tau)$ de dos señales temporales $\mathrm{u}_{1}(\mathrm{t})$ y $\mathrm{u}_{2}(\mathrm{t})$ se define como sigue:

$$
C(\tau)=u_{1}(t) * u_{2}(t)=\int_{-\infty}^{\infty} u_{1}(t) u_{2}(t+\tau) d t
$$

siendo * el operador de correlación cruzada, t el tiempo, $\tau$ el tiempo de retraso o tiempo que se desplaza la función $\mathrm{u}_{2}(\mathrm{t})$ respecto a $\mathrm{u}_{1}(\mathrm{t})$ (Figura 5.5).

Se ha demostrado teóricamente que si se considera una distribución espacial aleatoria de fuentes de ruido, la correlación cruzada de la señal registrada en dos estaciones converge a la función de Green del medio que existe entre ellos (Weaver, 2005; Gouédard et al., 2008).

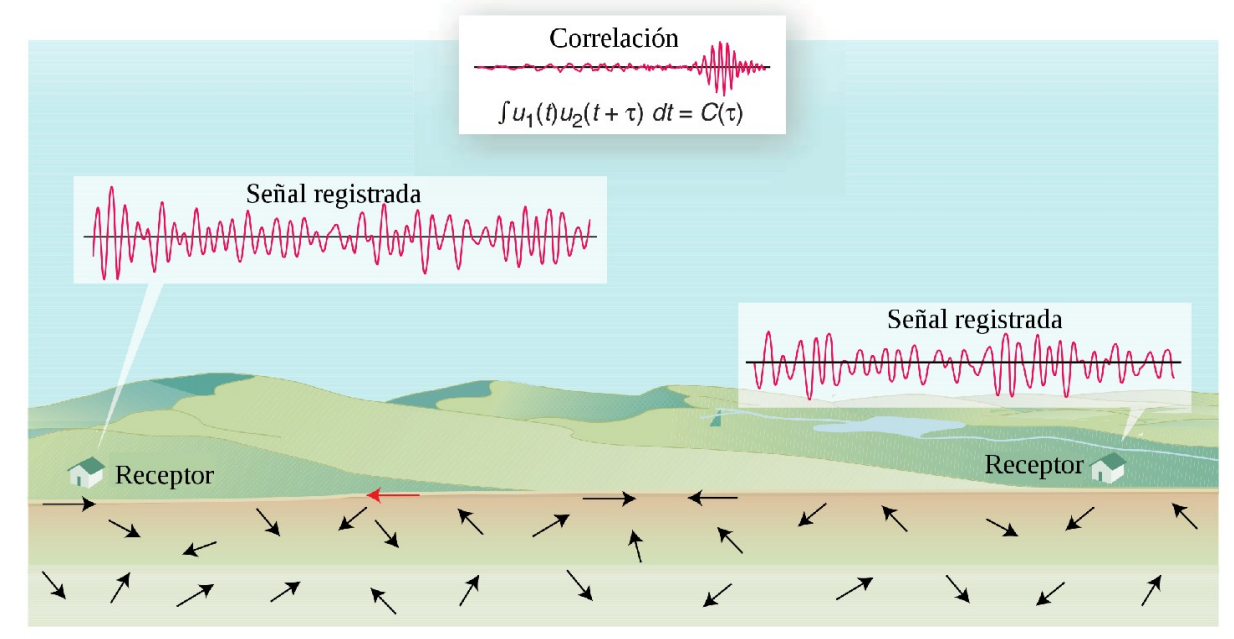

Figura 5.5: Ejemplo de registros de señales aleatorias en dos receptores ante la presencia de un campo de ondas difuso generado por fuentes distantes y/o por múltiple scattering. Además se muestra un rayo que ocasionalmente es registrado en ambas estaciones (flecha roja). En el panel de arriba se muestra la correlación de ambas señales registradas. (Extraída y modificada de Weaver, 2005).

La correlación cruzada de los registros de ruido ambiente entre dos estaciones está compuesta 
por una parte con tiempo de retraso positivo y otra con tiempo de retraso negativo, que comúnmente se llaman parte causal y acausal, respectivamente.

Debido a que el ruido sísmico proviene de todas las direcciones, los registros contendrán energía que ha viajado en ambas direcciones. Por consiguiente, las funciones de Green que resulten de la correlación cruzada de ruido ambiente contendrán energía en la parte causal y acausal siendo simétricas respecto de un tiempo de retraso cero cuando la cantidad de energía que se propaga en ambas direcciones es la misma.

En la Figura 5.6 se observa la parte causal y acausal de la correlación cruzada entre dos estaciones de TdF.
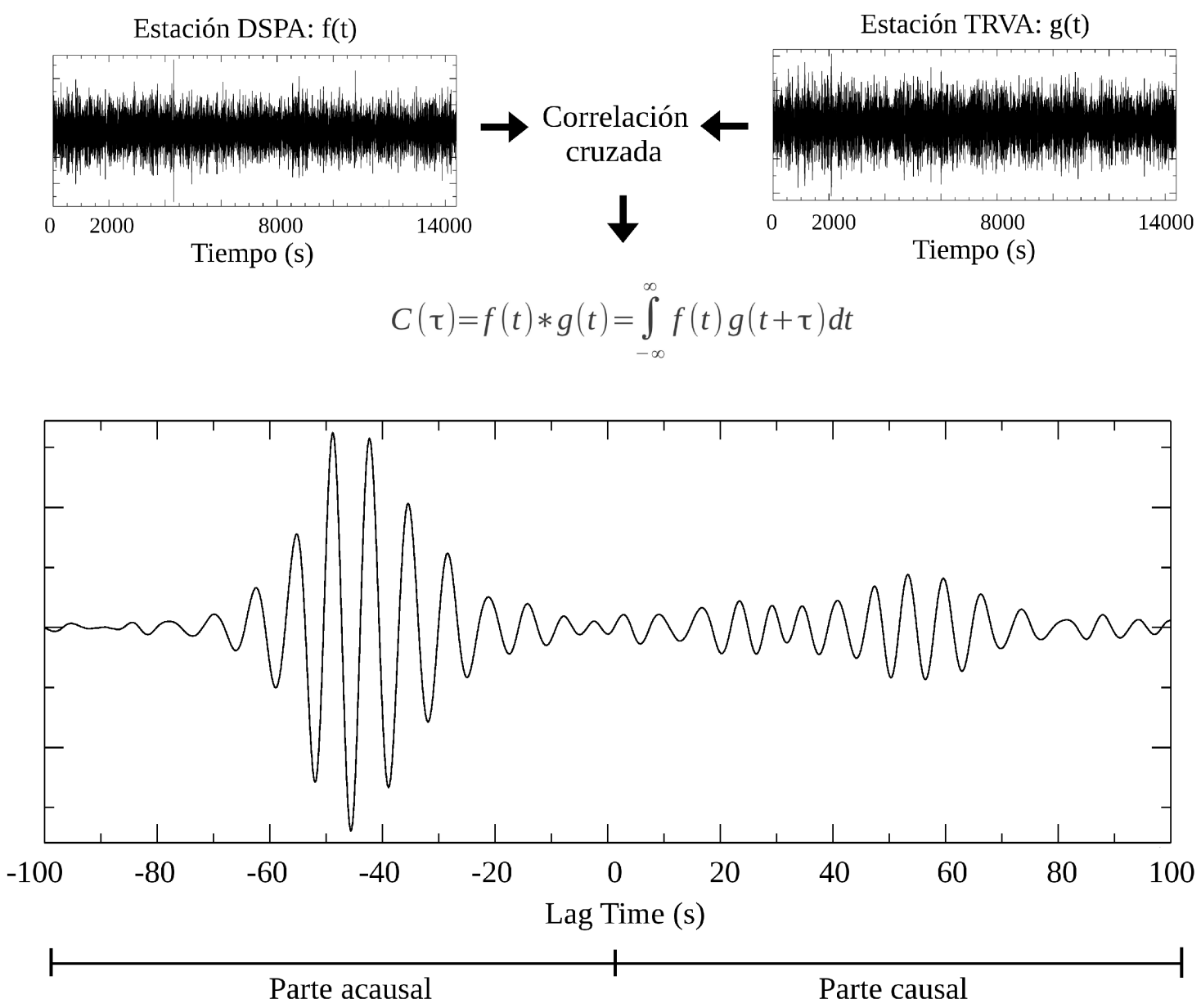

Figura 5.6: Ejemplo de la componente vertical de dos registros de ruido sísmico obtenidos en las estaciones DSPA y TRVA y la correspondiente correlación cruzada de los mismos. En este ejemplo, se observa que la mayor cantidad de energía debida a las ondas superficiales corresponde a un tiempo de retraso negativo. De esta manera, se puede concluir que en este caso, la energía se propaga desde TRVA a DSPA ya que la correlación se realizó de DSPA a TRVA con un tiempo de retraso negativo. 
La parte causal representa la energía que llega a la estación TRVA como respuesta a una fuente impulsiva en la estación DSPA, mientras que la parte acausal representa la energía que llega a la estación DSPA como respuesta a una fuente ubicada en la estación TRVA. Asumiendo que las funciones de Green son las mismas en ambas direcciones, la parte causal y acausal contienen la misma información pero en tiempo reverso. En la práctica esto no ocurre porque generalmente el campo ondulatorio no es isótropo ya que las fuentes no están distribuidas de forma homogénea y por lo tanto la energía no es la misma en todas las direcciones. Debido a esto la correlación cruzada de dos registros de ruido sísmico suele ser asimétrica. Teniendo en cuenta la definición de la parte causal y acausal de una correlación es posible conocer desde qué receptor provienen las ondas más energéticas considerando el tiempo de retraso positivo y negativo.

a
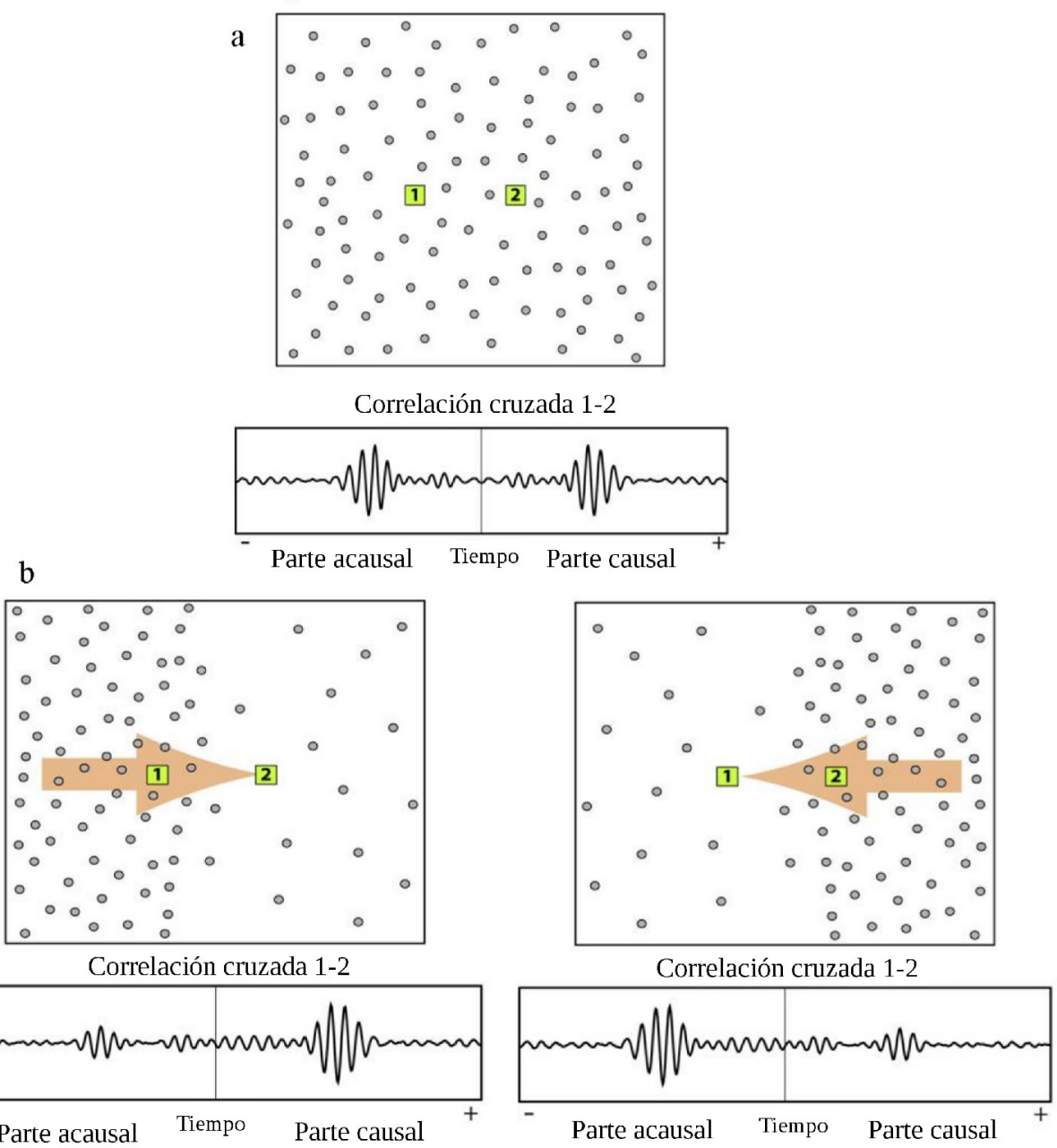

Figura 5.7: Ilustración del efecto en la correlación cruzada entre dos receptores 1 y 2 ante la presencia de una distribución de fuentes de ruido sísmico a) homogénea y b) no homogénea. En el primer caso (a) la correlación cruzada es simétrica respecto del tiempo de retraso 0, mientras que en el segundo caso (b) la correlación cruzada no es simétrica (pero si las curvas de tiempo de recorrido), lo cual está asociado a una distribución anisótropa de las fuentes. (Extraída y modificada de Stehly et al., 2006). 
Si las fuentes de ruido están distribuidas homogéneamente en el espacio, la correlación cruzada es simétrica respecto del tiempo de retraso 0. Es decir la parte causal y acausal son idénticas (Figura 5.7a). En la práctica y con datos de campo, esto no suele ocurrir y generalmente la parte negativa y positiva de la correlación cruzada entre un par de estaciones no es igual (Figura 5.8). En este último caso, se calcula la media entre la parte causal y la acausal con el fin de obtener lo que se denomina señal o correlación simétrica. El cálculo de la señal simétrica promedia el efecto de fuentes de ruido distribuidas en forma no homogénea e incrementa la energía de fases coherentes frente a aquella de las señales incoherentes. Normalmente la extracción de información de ondas superficiales, estimando sus velocidades, se suele realizar considerando la señal simétrica.
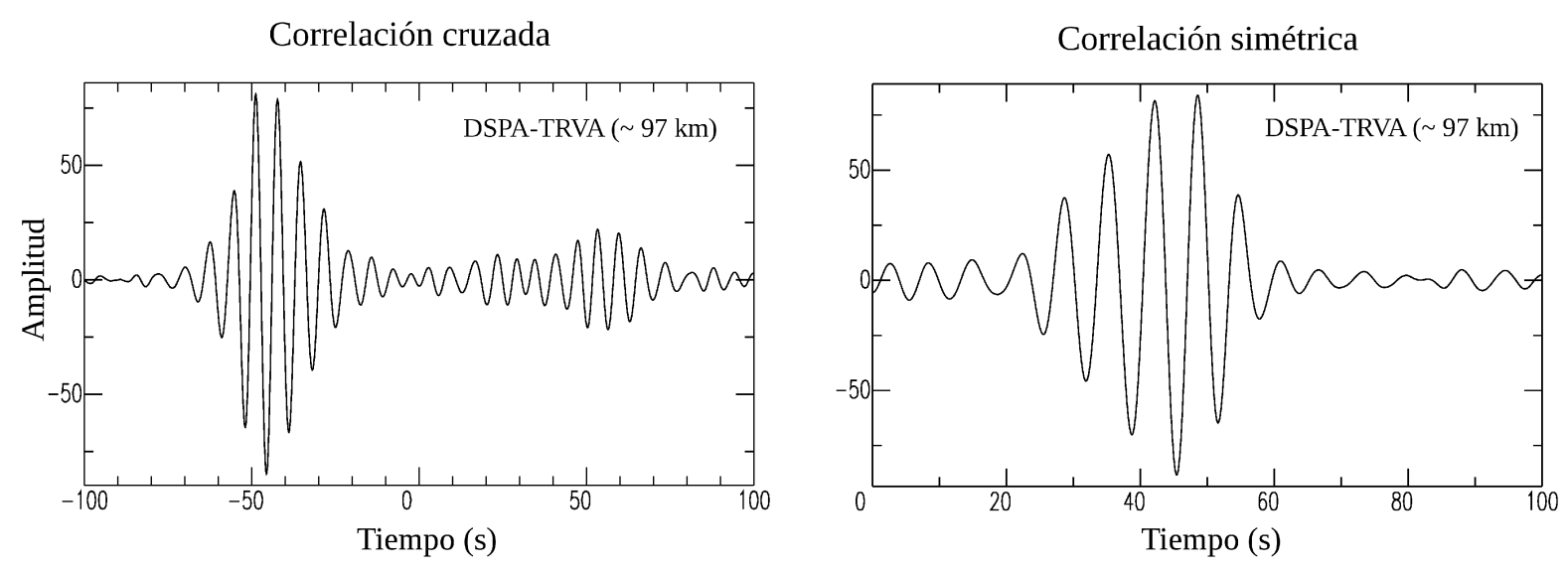

Figura 5.8: Correlación cruzada de ruido sísmico registrado en las estaciones DSPA y TRVA (izquierda) y la correspondiente correlación simétrica (derecha) como resultado de realizar la media entre la parte causal y acausal de la correlación cruzada. Para el cálculo se utilizó la componente vertical. La distancia entre ambas estaciones en este ejemplo es $97 \mathrm{~km}$.

\subsubsection{2 ¿Qué es la función de Green?}

La función de Green se define como la respuesta del medio a una fuerza impulsiva y su estimación permite predecir el comportamiento del suelo para una cierta localización frente a ciertas perturbaciones.

Con el fin de obtener las funciones completas de Green, las fuentes que generan ruido sísmico deben estar distribuidas uniformemente en el subsuelo (Wapenaar, 2004). El ruido sísmico proveniente del océano no está uniformemente distribuido y está compuesto en su mayoría por ondas cuya dirección de propagación es paralela a la superficie terrestre (ondas tipo Rayleigh y Love). Sin embargo, se ha demostrado que es posible reconstruir parcialmente la función de Green del medio entre dos receptores considerando la energía correspondiente a las ondas superficiales que se propagan entre ellos (Lobkis y Weaver, 2001; Derode et al., 2003). 


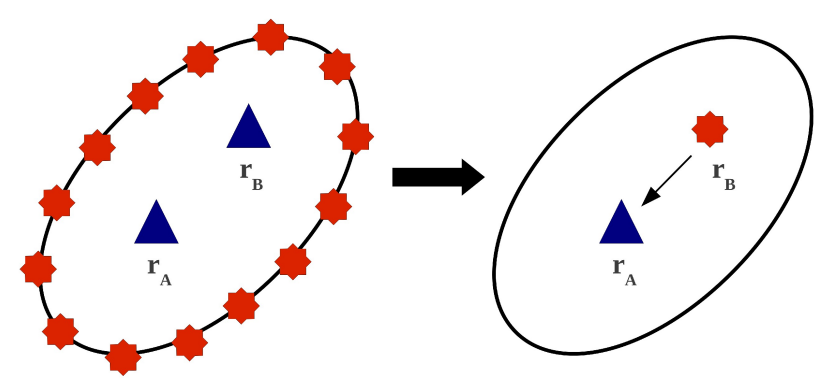

Figura 5.9: Esquema de una distribución de fuentes de ruido sísmico (estrellas rojas) alrededor de dos estaciones A y B con posición $\mathrm{r}_{\mathrm{A}}$ y $\mathrm{r}_{\mathrm{B}}$ (izquierda) y su representación según el concepto de la interferometría sísmica (derecha) en el que se considera que se puede obtener la función de Green del medio a partir de la correlación cruzada entre las estaciones A y B como si la fuente estuviera ubicada en una de las estaciones (fuente virtual).

El concepto de la interferometría sísmica se basa en el concepto de fuente virtual (Figura 5.9). Es decir, mediante la correlación cruzada de registros de ruido sísmico de dos estaciones, es posible construir el campo de ondas que registraría una de las estaciones si consideramos una fuente ubicada en la otra estación y viceversa (Rickett y Claerbout, 1999).

La correlación cruzada de trazas de ruido sísmico $\mathrm{C}_{\mathrm{AB}}(\mathrm{t})$ está relacionada con la función de Green del medio mediante la siguiente ecuación (Lobkis y Weaver, 2001; Roux et al., 2005), siendo $\mathrm{G}_{\mathrm{AB}}$ la función de Green del medio entre las estaciones A y B:

$$
\frac{d C_{A B}(t)}{d t} \approx-G_{A B}(t)+G_{A B}(-t) \quad-\infty<t<\infty
$$

donde $\mathrm{C}_{\mathrm{AB}}(\mathrm{t})$ y $\mathrm{C}_{\mathrm{AB}}(-\mathrm{t})$ es la parte causal y acausal de la correlación cruzada entre las estaciones $\mathrm{A}$ y $\mathrm{B}$, respectivamente.

Según Lin et al. (2008):

$$
G_{A B}(t)=\frac{-d C_{A B}(t)}{d t} \quad \text { y } \quad G_{B A}(t)=\frac{-d C_{A B}(-t)}{d t} \quad 0 \leq t<\infty
$$

Y considerando el teorema de reciprocidad espacial de las funciones de Green:

$$
G_{A B}(t)=G_{B A}(t)
$$


se obtiene la correlación simétrica como la media entre la parte causal y acausal.

De esta manera, es posible calcular la función de Green del medio en el que se propagaron las ondas superficiales entre dos estaciones a partir de la correlación simétrica de las trazas temporales de ruido sísmico registrado en las dos estaciones:

$$
G_{A B}(t)=\frac{-d}{d t}\left[\frac{C_{A B}(t)+C_{A B}(-t)}{2}\right] \quad 0 \leq t<\infty
$$

\subsection{Aplicación de la técnica en TdF}

\subsubsection{Preprocesamiento de los datos}

Con el fin de calcular las correlaciones cruzadas de ruido sísmico en TdF, los datos fueron preprocesados tomando en cuenta los siguientes pasos:

Extracción de los datos y conversión: Se realizó la extracción del año 2008 de la base de datos de las estaciones sismológicas de banda ancha: DSPA, TRVA, ELCA y USHA cuya localización de muestra en el mapa de la Figura 1.2 del Capítulo I. El formato original de los mismos Güralp, Seed y Miniseed fue convertido a SAC (Goldstein y Snoke, 2005) considerando la componente vertical y eliminando aquellos registros que tuvieran irregularidades o gaps con el fin de tener registros continuos para la posterior correlación cruzada. Además, los datos estaban almacenados en registros de 1 hora, por lo que se prepararon registros de 24 hs.

$>$ Remoción la respuesta de instrumento: Para poder comparar los datos registrados en estaciones sismológicas equipadas con diferentes instrumentos, es necesario eliminar la respuesta del instrumento en cada registro. Este proceso consiste en realizar la deconvolución del registro con la respuesta del instrumento en la banda de frecuencias de interés. Las cuatro estaciones utilizadas en este trabajo están equipadas con instrumentos diferentes por lo que se calculó la respuesta de cada uno y fueron removidas de los datos mediante el software SAC.

Remoción de la media y la pendiente: Este paso es muy común en el tratamiento de señales sísmicas y se suelen realizar dentro del software SAC con los comandos "rmean" y “rtrend”. La reducción de la media consiste en desplazar la línea media del registro a nivel cero mientras que la reducción de pendiente consiste en eliminar del registro cualquier 
sesgo con el fin de obtener una media horizontal. Este paso del preprocesado de señales sísmicas fue explicado también en la sección 2.4.2.2 del Capítulo II.

Selección de la ventana de tiempo y corte de los extremos: Cuando los datos son filtrados en un rango determinado de frecuencias, la forma de onda se ve afectada disminuyendo su amplitud en los extremos del registro. Para eliminar este efecto es necesario cortar los extremos del registro luego de realizar la deconvolución del mismo con la respuesta del instrumento. Es fundamental realizar este paso antes de decimar el registro, ya que el corte puede introducir un error al tiempo inicial que es proporcional al intervalo de muestreo, y al decimar este intervalo aumenta.

Decimación: El proceso de decimación se suele realizar con el fin de reducir el tiempo de cómputo y aumentar la eficiencia. El mismo consiste en reducir el número de muestras del registro. Los datos originales estaban compuestos de registros con $20 \mathrm{mps}$, lo que significa una frecuencia de Nyquist de $10 \mathrm{~Hz}$, suficiente para la extracción de ondas superficiales. Pero con el fin de reducir el tiempo de cálculo, los datos fueron decimados a $10 \mathrm{mps}$ luego del paso anterior.

Filtrado Pasabanda: Los datos fueron filtrados con un pasabanda cuya frecuencia máxima fue fijada al valor de $0.4 \mathrm{~Hz}$, mientras que las frecuencias inferiores se ajustaron de manera que la distancia entre cada par de estaciones corresponda, al menos, a tres longitudes de onda. Los valores de la frecuencia mínima así están comprendidas en el rango $0.06-0.12 \mathrm{~Hz}$, dependiendo las estaciones sismológicas utilizadas en el cálculo.

\subsubsection{Cálculo de las correlaciones cruzadas}

Luego del preprocesamiento de los datos, se calcularon las correlaciones cruzadas entre pares de estaciones. En el presente trabajo se seleccionaron datos de $n=4$ estaciones sismológicas de banda ancha, esto significa que se obtendrán $n(n-1) / 2=6$ funciones de Green o trayectos de ondas superficiales.

Se aplicó la técnica conocida como phase cross-correlation (PCC) que se basa en la coherencia de fase de señales analíticas (Schimmel, 1999).

La señal analítica $s(\mathrm{t})$ de una serie temporal $u(\mathrm{t})$ se define como:

$$
s(t)=u(t)+i H[u(t)]
$$


siendo $\mathrm{H}[\mathrm{u}(\mathrm{t})]$ la transformada de Hilbert de la serie temporal $\mathrm{u}(\mathrm{t})$.

La forma exponencial de la señal analítica s(t) se puede escribir como:

$$
s(t)=a(t) \exp (i \Phi(t))
$$

siendo $a(\mathrm{t})$ la envolvente y $\Phi(\mathrm{t})$ la fase instantánea.

De esta manera la PCC queda definida de la siguiente manera:

$$
c_{p c c}(t)=\frac{1}{2 \mathrm{~T}} \sum_{\tau=\tau_{0}}^{\tau_{0}+T}\left\{\left(\left|e^{i \Phi(t+\tau)}+e^{i \Psi(\tau)}\right|\right)^{v}-\left(\left|e^{i \Phi(t+\tau)}-e^{i \Psi(\tau)}\right|\right)^{v}\right\}
$$

En este caso $\mathrm{c}_{\mathrm{pcc}}$ mide la similitud de dos series temporales $\mathrm{u}_{1}$ (primer paréntesis) y $\mathrm{u}_{2}$ (segundo paréntesis) como funciones del tiempo de retraso. Las fases instantáneas de $\mathrm{u}_{1} \mathrm{y} \mathrm{u}_{2} \operatorname{son} \Phi(\tau)$ y $\Psi(\tau)$, respectivamente. La sensibilidad de $c_{p c c}(t)$ se puede aumentar utilizando la potencia $v>1$. En este trabajo y con los datos utilizados fue suficiente considerar $v=1$. Además la norma de PCC es $\left|\mathrm{C}_{\mathrm{pcc}}\right|$ $\leq 1$, indicando una perfecta correlación cuando $\mathrm{c}_{\mathrm{pcc}}=1 \mathrm{y}$ una anticorrelación cuando $\mathrm{c}_{\mathrm{pcc}}=-1$.

Por otro lado se calculó además la correlación cruzada convencional pero los resultados obtenidos con PCC demostraron ser más eficientes al momento de extraer infromación de ondas Rayleigh en la zona de estudio.

\subsubsection{Apilamiento de las correlaciones cruzadas}

La presencia de señal coherente frente a la de señal incoherente en una correlación, se puede incrementar sumando o apilando las correlaciones cruzadas calculadas con registros en diferentes rangos de tiempo. Pero esto a menudo dependerá de la ubicación de la estación, de la época del año, del azimut entre el par de estaciones así como también de la distribución de las fuentes. Generalmente cuanto mayor sea el número de correlaciones, mejor se verá la señal coherente (Bensen et al., 2007).

El apilamiento o stack es la suma de las correlaciones cruzadas calculadas con trazas registradas en diferentes rangos de tiempo para el mismo par de estaciones. Además de aumentar la SNR, este procedimiento se lleva a cabo con el fin de aproximar el caso al de una distribución isótropa de ruido sísmico. El espectro de ruido sísmico está dominado por los microsismos primario y secundario, cuyo origen está vinculado con los océanos y costas, como ya se ha mencionado en la sección 5.4.1, y varían según la época del año. Al apilar las correlaciones cruzadas en distintas 
épocas del año, se promedia las variaciones de los cambios de forma que pueden surgir por la variabilidad estacional, considerando una distribución de las fuentes de ruido más homogénea y de esta manera se puede obtener la función de Green del medio.

Se utilizaron dos tipos diferentes de apilamiento. El primero consiste en realizar la suma lineal de las correlaciones cruzadas en el dominio del tiempo conocido como stack lineal. Mediante este proceso, la amplitud de las fases coherentes con más energía aumenta incrementando la relación de señal coherente frente a la señal incoherente. La desventaja de este método es que las fases coherentes con poca energía no aumentan su amplitud de la misma manera que lo hacen las que tienen más energía.

Por otro lado, se aplicó también un apilamiento en el dominio del tiempo y de la frecuencia conocido como time-frequency domain PWS (tf-PWS) propuesto por Schimmel et al. (2011). Esta técnica realiza la suma de todas las fases coherentes independientemente de la amplitud basándose en el método phase weighted stack (PWS) desarrollado por Schimmel y Paulssen (1997). El PWS es una suma no-lineal en el que se asigna un peso a cada muestra mediante una medida de coherencia.

La técnica propuesta por Schimmel et al. (2011) se basa en descomponer la señal en el dominio tiempo-frecuencia mediante la representación analítica de la transformada S (Stockwell el al., 1996). De esta manera, el apilamiento de fase en el dominio de tiempo-frecuencia está definido como:

$$
c_{p s}(\tau, f)=\left(\mid \frac{1}{N} \sum_{j=1}^{N}\left(\frac{S_{j}(\tau, f) e^{i 2 \Pi f \tau}}{\left|S_{j}(\tau, f)\right|}\right)\right)^{v}
$$

siendo $\mathrm{S}_{\mathrm{j}}(\tau, \mathrm{f})$ la transformada $\mathrm{S}$ de la j-ésima serie temporal (en este caso, correlación cruzada) y $\mathrm{N}$ es el número de trazas o muestras. Es decir que para calcular $\mathrm{c}_{\mathrm{ps}}(\tau, \mathrm{f})$ cada traza individual debe ser transformada al dominio tiempo-frecuencia. El parámetro $v$ ajusta la transición entre señal coherente y menos coherente en la suma. Aquí se utiliza v=1. Por consiguiente el apilamiento $\mathrm{S}_{\mathrm{pws}}(\tau, \mathrm{f})$ se obtiene mediante la multiplicación en el dominio tiempo-frecuencia de la coherencia de fase $\mathrm{C}_{\mathrm{ps}}(\tau, \mathrm{f})$ y la transformada $\mathrm{S}$ de la suma lineal de todas las correlaciones $\mathrm{S}_{\mathrm{ls}}(\tau, \mathrm{f})$ :

$$
S_{p w s}(\tau, f)=c_{p s}(\tau, f) S_{l s}(\tau, f)
$$

Luego se aplica la transformada S inversa (Schimmel y Gallart, 2005; Simon et al., 2007) con el fin de transformar $\mathrm{S}_{\mathrm{pws}}(\tau, \mathrm{f})$ al dominio del tiempo $\mathrm{S}_{\mathrm{pws}}(\mathrm{t})$ para su interpretación. 


\subsection{Resultados y discusión}

En la Figura 5.10 se compara el stack lineal (t-lin) y el phase weighted stack (tf-PWS) considerando las correlaciones cruzadas TRVA-USHA y DSPA-TRVA. En ambos casos, los dos tipos de apilamiento mejoran la relación señal-ruido y se observa la emergencia para una onda tipo Rayleigh. Sin embargo, la aplicación de la técnica PCC demostró ser suficiente para la extracción de las señales en este trabajo. En lo que sigue se utiliza el stack lineal de las correlaciones cruzadas para extraer información del medio no siendo necesaria la aplicación del tf-PWS.
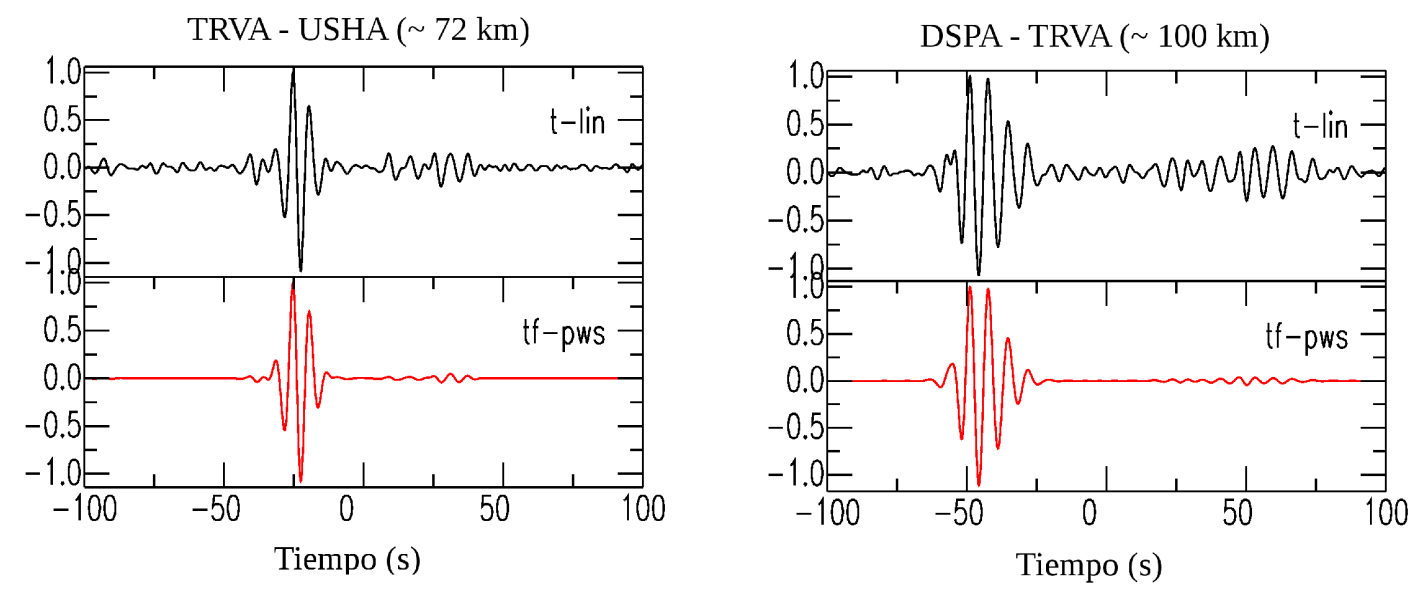

Figura 5.10: Ejemplo de los dos tipos de apilamiento de la correlación cruzada (PCC) de ruido sísmico (1 año de datos) de las estaciones TRVA-USHA (izquierda, la distancia entre estaciones es 72 km), y DSPA-TRVA

(derecha, la distancia entre estaciones es $100 \mathrm{~km}$ aproximadamente). El eje vertical indica la amplitud normalizada. Las trazas en los paneles superiores (en negro) fueron obtenidas mediante el stack lineal (t-lin), mientras que los paneles inferiores (en rojo) corresponden a la aplicación del phase weighted stack (tf-PWS).

Las correlaciones cruzadas fueron calculadas aplicando el método PCC. En la Figura 5.11 se muestra el apilamiento lineal de un año de datos de las correlaciones cruzadas realizadas entre todos los pares de estaciones disponibles de TdF. En todos los casos se observa la emergencia de ondas superficiales para tiempos de retraso negativos. Es decir que en todos los casos, la mayor cantidad de energía proviene de estaciones ubicadas al sur ya que la primer estación considerada en cada cálculo está ubicada al norte de la segunda estación y por lo tanto las ondas que provienen del sur se manifiestan con un tiempo de retraso negativo (la ubicación de las estaciones se muestra en la Figura 1.2 del Capítulo I).

Las formas de onda obtenidas a partir de la correlación cruzada de ruido ambiente son señales dominadas por los picos microsísmicos en las bandas de frecuencia $0.05-0.10$ y $0.1-0.2 \mathrm{~Hz}$, y los microsismos se propagan predominantemente como ondas Rayleigh (LaCoss et al., 1969; Friedrich 
et al., 1998; Bromirsky, 2001; Bromirski y Duennebier, 2002; Stehly et al., 2006; Stutzmann et al., 2012).

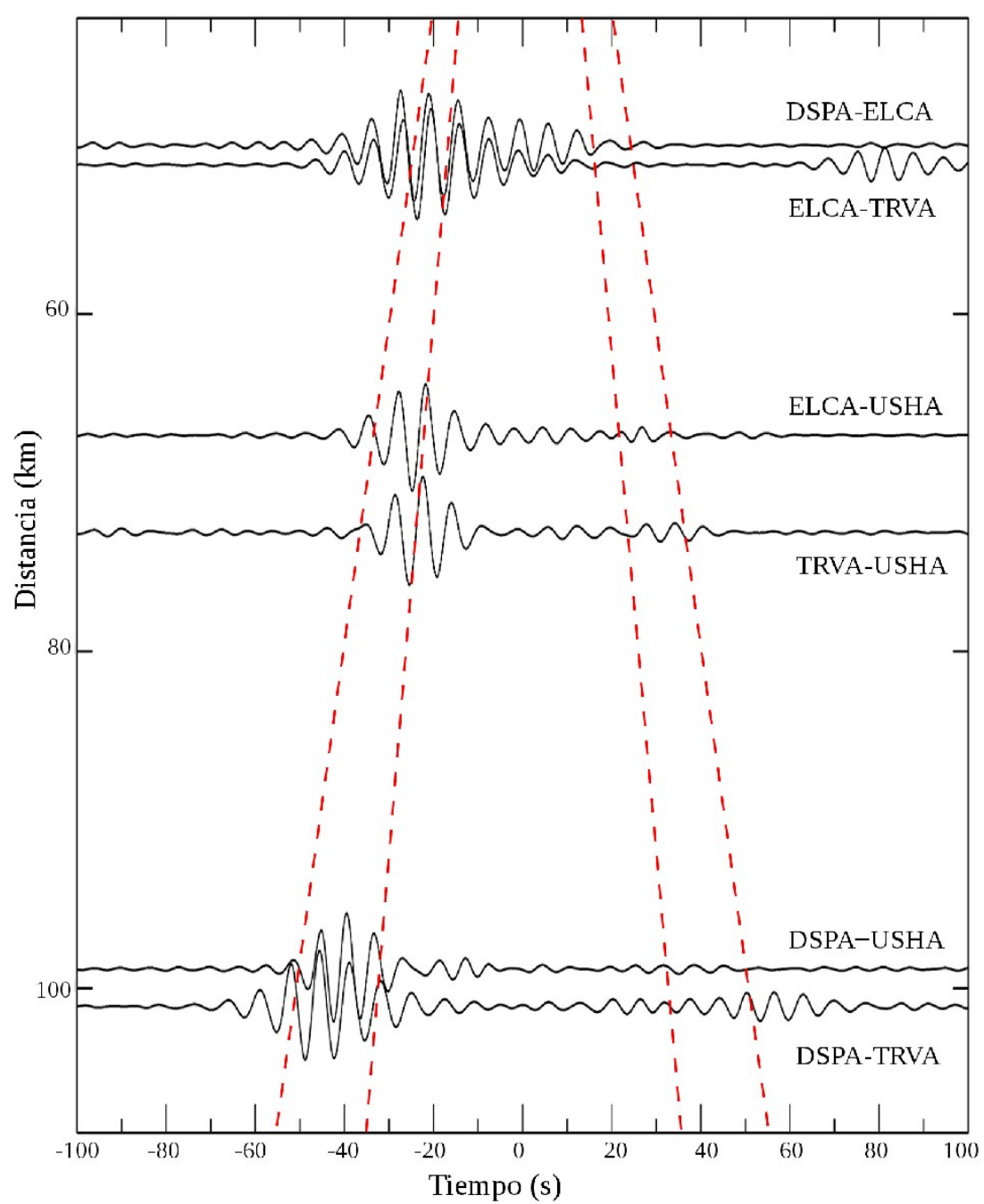

Figura 5.11: Apilamiento lineal de las correlaciones cruzadas (PCC) de registros de ruido sísmico obtenidos en las estaciones de banda ancha de TdF cuya localización puede verse en la Figura 1.2 del Capítulo I. Las líneas punteadas rojas indican las curvas de tiempo de recorrido con pendientes v=2 km/s y v=3 km/s que representan valores típico de propagación de ondas superficiales en los períodos considerados. El eje vertical indica la distancia entre las estaciones utilizadas en cada correlación cruzada.

Con el fin de calcular las curvas de dispersión, se calculó además la correlación simétrica descripta en la sección 5.1.5.2 (Figura 5.12). En el capítulo siguiente se calculan las curvas de dispersión considerando dichas correlaciones simétricas con el fin de extraer información de la estructura media entre cada par de estaciones. 

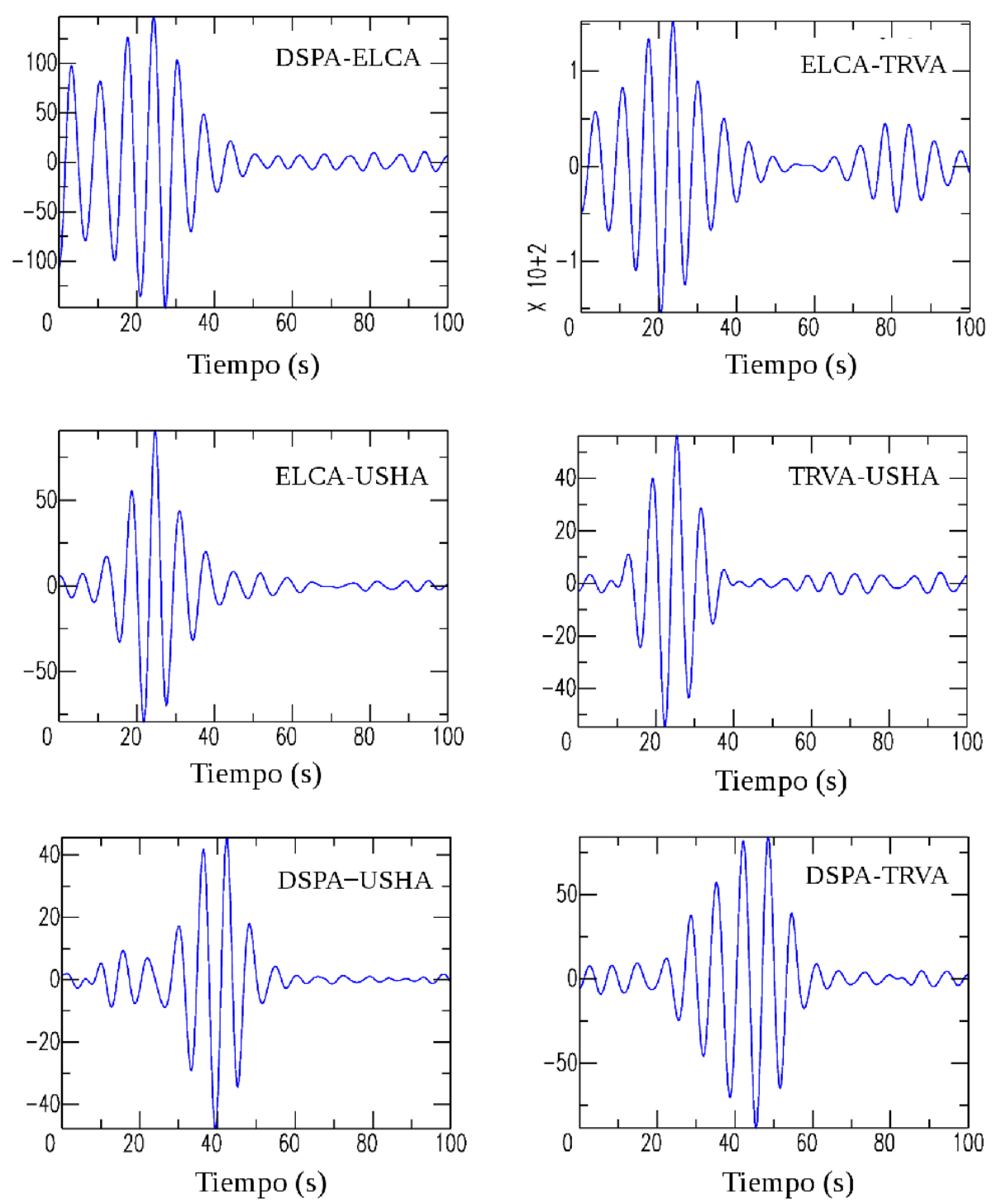

Figura 5.12: Correlación simétrica obtenida a partir de las correlaciones cruzadas observadas en la Figura 5.11. Se consideró esta señal simétrica para el posterior cálculo de curvas de dispersión.

En la práctica, cuando se trabaja con registros reales de ruido sísmico, las correlaciones cruzadas obtenidas entre pares de estaciones no son simétricas respecto de un tiempo de retraso cero. Esto ocurre porque el campo ondulatorio del ruido sísmico no es difuso o acimutalmente isótropo debido a la distribución geográfica de las fuentes sísmicas (Mulargia, 2012). A pesar de esto, es posible obtener información de la función de Green del medio considerando ciertas características del ruido sísmico:

1) El ruido sísmico está compuesto principalmente por ondas superficiales. Esto se debe a que la fuente principal de generación del ruido está cerca de la superficie (Snieder, 2004; Kedar et al., 
2008). Los receptores y las fuentes están en la superficie y las ondas superficiales son más energéticas por experimentar menor atenuación (geometrical spreading). Por consiguiente el ruido sísmico oceánico está compuesto principalmente por ondas superficiales Rayleigh y Love, las cuales se propagan paralelamente a la superficie terrestre.

2) El scattering de las ondas y el apilamiento de las correlaciones cruzadas de diferentes épocas favorecen la homogeneidad de la distribución de las fuentes de ruido sísmico. De esta manera, se puede obtener una función de Green del medio a pesar que el ruido sísmico sea un campo anisótropo.

3) La correlación cruzada de registros de ruido sísmico entre pares de estaciones está principalmente dominada por la contribución de las fuentes localizadas en la proximidad de la línea que conecta las estaciones (Snieder, 2004). Teniendo en cuenta esto, la presencia de una cantidad suficiente de fuentes de ruido localizadas favorablemente permite la obtención de ondas superficiales que se propagan entre las dos estaciones. 


\section{Obtención de curvas de dispersión a partir de ruido sísmico: Cálculo e inversión}

\subsection{Introducción}

La implementación del cálculo de las correlaciones cruzadas de ruido sísmico entre pares de estaciones fue uno de los grandes avances dentro de la sismología con el fin de estudiar la estructura interna de la Tierra. Como se mencionó en el capítulo anterior, esto fue posible mediante la obtención de la función empírica de Green del medio que separa un par de estaciones (Lobkis y Weaver, 2001; Shapiro y Campillo, 2004). A partir de dicha función es posible extraer información de las ondas superficiales que se propagan en el medio entre las dos estaciones y así calcular curvas de dispersión que conservan información del medio. Es decir, a partir de las correlaciones cruzadas de registros de ruido ambiente es posible extraer información acerca de la dispersión de ondas superficiales calculando velocidades de grupo y fase.

Este tipo de ondas son una herramienta de gran utilidad para estudiar la estructura interna de la Tierra ya que presentan el fenómeno de dispersión, es decir, su velocidad de propagación depende de la frecuencia y este concepto es utilizado para estimar las características del medio por el que se propagaron las ondas. Las ondas superficiales a diferentes períodos son sensibles ante la presencia de diversas estructuras en el subsuelo a diferentes profundidades. En general, a partir de períodos cortos se puede obtener información de capas superficiales, mientras que para períodos largos, las ondas superficiales son sensibles a capas más profundas. Calculando sus velocidades de propagación es posible realizar gráficos de velocidad en función del período. A partir de estos gráficos o curvas de dispersión, y mediante un proceso de inversión, es posible inferir ciertas características del medio por el cual se propagaron las ondas. 


\subsubsection{Ondas Rayleigh y velocidades de dispersión}

Existen dos tipos de ondas superficiales: las ondas Rayleigh y las ondas Love. Si se considera un medio elástico con una superficie libre, las ondas Rayleigh pueden generarse y propagarse a través del medio. En el caso de las ondas Love existen además otras restricciones para su formación. En este trabajo sólo se han obtenido ondas Rayleigh (modo fundamental) calculando sus velocidades de dispersión.

Cada una de las ondas superficiales que se propagan a lo largo de un semiespacio elástico lateralmente isótropo tiene un número infinito de modos: el modo fundamental de índice $\mathrm{k}=0 \mathrm{y}$ los modos superiores con $\mathrm{k}=1,2, \ldots$ Los modos involucran frecuencias finitas en las que el sistema puede vibrar. En el presente trabajo sólo se hace referencia al modo fundamental de las ondas Rayleigh, los modos superiores no fueron observados ni analizados.

En general, la profundidad a la cual las ondas Rayleigh son más sensibles es $\lambda / 2$, siendo $\lambda$ la longitud de onda (Dahlen y Tromp, 1998). Los periodos cortos ( $\mathrm{T} \leq 10 \mathrm{~s}$ ) generan información sobre estructuras corticales superficiales, mientras que los períodos mayores de ondas Rayleigh (por ej. $\mathrm{T}=30 \mathrm{~s}$ ) son sensibles a contrastes en la corteza inferior y al espesor de toda la corteza.

La medición de la velocidad de propagación de las ondas superficiales se puede realizar a través de dos medidas: la velocidad de fase y la velocidad de grupo. La primera se define como la velocidad con la que se propaga la fase c de cada componente armónico y se define como (Hofmann, 2015):

$$
c(w)=\frac{w}{k} \quad \text { Velocidad de fase }
$$

siendo w la frecuencia angular, $\mathrm{k}$ el número de onda definido por $k=\frac{2 \Pi}{\lambda}$ y $\lambda$ la longitud de onda.

Por otro lado, la velocidad de grupo u se define como la velocidad de la onda envolvente que transporta la energía y se define como:

$$
u(w)=\frac{d w}{d k} \quad \text { Velocidad de grupo. }
$$

La velocidad de grupo y de fase son principalmente sensibles a la velocidad de onda S en función de la profundidad y del período. 


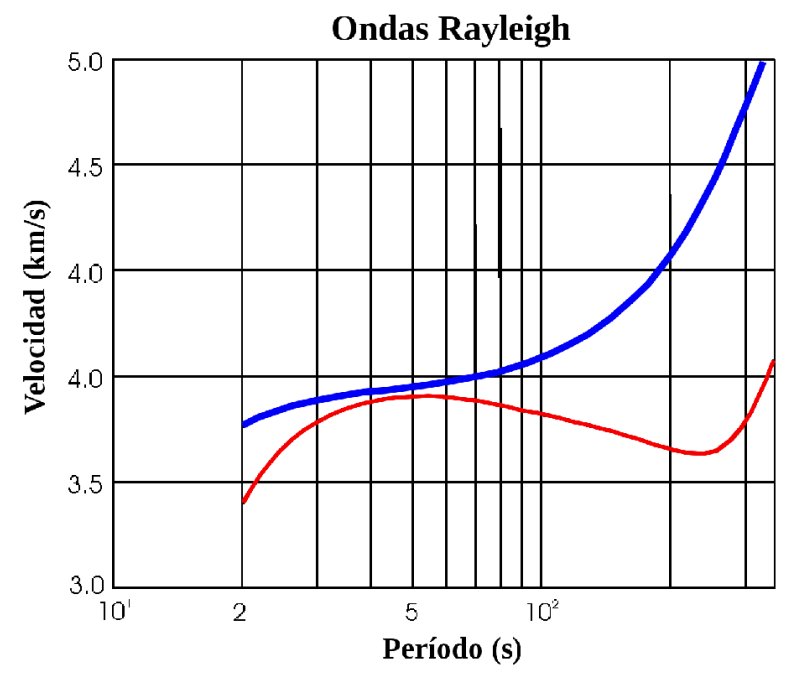

Figura 6.1: Curvas de dispersión correspondiente a las velocidades de grupo (en rojo) y de fase (en azul) del modo fundamental de ondas Rayleigh realizado para el modelo PREM (Dziewonski y Anderson, 1981).

(Extraído y modificado de Bormann, 2002).

\subsection{Cálculo de las curvas de dispersión}

El presente trabajo se orientó a medir las velocidades de grupo de ondas Rayleigh a partir de correlaciones cruzadas de registros de ruido sísmico entre pares de estaciones.

Para medir las velocidades de grupo es necesario calcular la representación en tiempofrecuencia de las correlaciones cruzadas. Dziewonski et al. (1969) calcula la representación en tiempo-frecuencia con ventanas Gaussianas. En este trabajo se utilizó la transformada S desarrollada por Stockwell et al. (1996) que es similar a la metodología realizada por Dziewonski et al. (1969). La transformada S de la función h(t) se define como:

$$
S(\tau, f)=\int_{-\infty}^{\infty} h(t) \frac{|f|}{\sqrt{2 \Pi}} \mathrm{e}^{-\frac{(\tau-t)^{2} f^{2}}{2}} \mathrm{e}^{-i 2 \Pi f t} d t
$$

siendo $\mathrm{f}$ la frecuencia, y S el operador transformada a partir del cual es posible recuperar la señal h(t) a partir de su transformada de Fourier H(f) (Stockwell et al., 1996).

Las velocidades de grupo se calcularon para el rango de frecuencias indicado en la Tabla 6.1. La máxima frecuencia fue fijada al valor de $0.4 \mathrm{~Hz}$, mientras que las frecuencias inferiores se ajustaron de manera que la distancia entre cada par de estaciones corresponda, al menos, a tres longitudes de onda. El rango de distancias entre estaciones varía desde 50 a $100 \mathrm{~km}$. Las frecuencias utilizadas en este estudio indican que las ondas Rayleigh tendrán una sensibilidad de 
estructuras en las capas más superficiales de la corteza.

\begin{tabular}{ccc}
\hline \hline Correlación cruzada & $\mathbf{f}_{\mathbf{1}} \mathbf{( H z )}$ & $\mathbf{f}_{\mathbf{2}} \mathbf{( H z )}$ \\
\hline \hline DSPA-ELCA & 0.12 & 0.4 \\
TRVA-ELCA & 0.12 & 0.4 \\
ELCA-USHA & 0.10 & 0.4 \\
TRVA-USHA & 0.08 & 0.4 \\
DSPA-USHA & 0.06 & 0.4 \\
DSPA-TRVA & 0.07 & 0.4 \\
\hline \hline
\end{tabular}

Tabla 6.1: Frecuencias utilizadas en el cálculo de las curvas de dispersión de ondas Rayleigh. Se indican la frecuencia inferior $\left(\mathrm{f}_{1}\right)$ y la frecuencia superior o máxima $\left(\mathrm{f}_{2}\right)$ que coincide en todos los casos y que han sido seleccionadas según lo mencionado en la sección 6.2 .

Mediante la representación en tiempo-frecuencia de las correlaciones cruzadas, utilizando la transformada S, las velocidades de grupo son luego medidas a partir de los máximos del espectro de amplitud de dicha representación (Figuras 6.2 y 6.3). Estas llegadas en tiempo-frecuencia se transforman en velocidad de grupo considerando la distancia entre cada par de estaciones
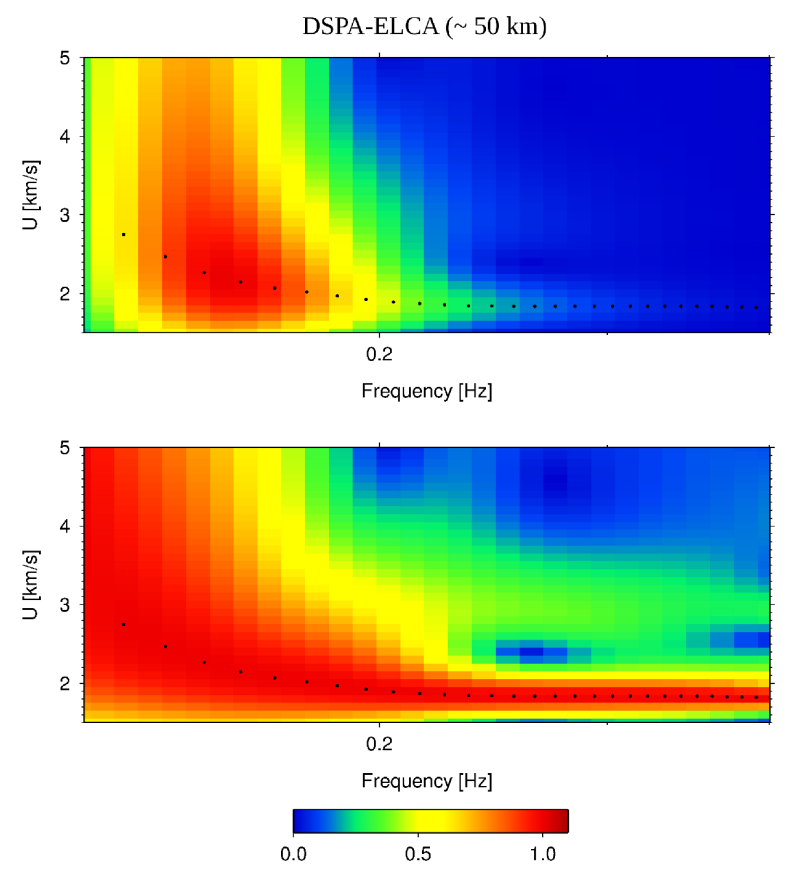

Figura 6.2: Velocidades de grupo obtenidas a partir de la correlación cruzada de los registros de ruido sísmico 
obtenido en DSPA-ELCA. Los puntos negros representan la velocidad de grupo en función de la frecuencia. Para el cálculo se utilizó el apilamiento lineal de la correlación cruzada (PCC) de un año de datos. El gráfico superior demuestra la distribución de energía normalizada a uno. En el gráfico inferior se normalizó la energía de cada frecuencia a uno para visualizar la llegada de grupo o energía. El eje de las frecuencias es logarítmico. La distancia entre las estaciones DSPA y ELCA es $50 \mathrm{~km}$.
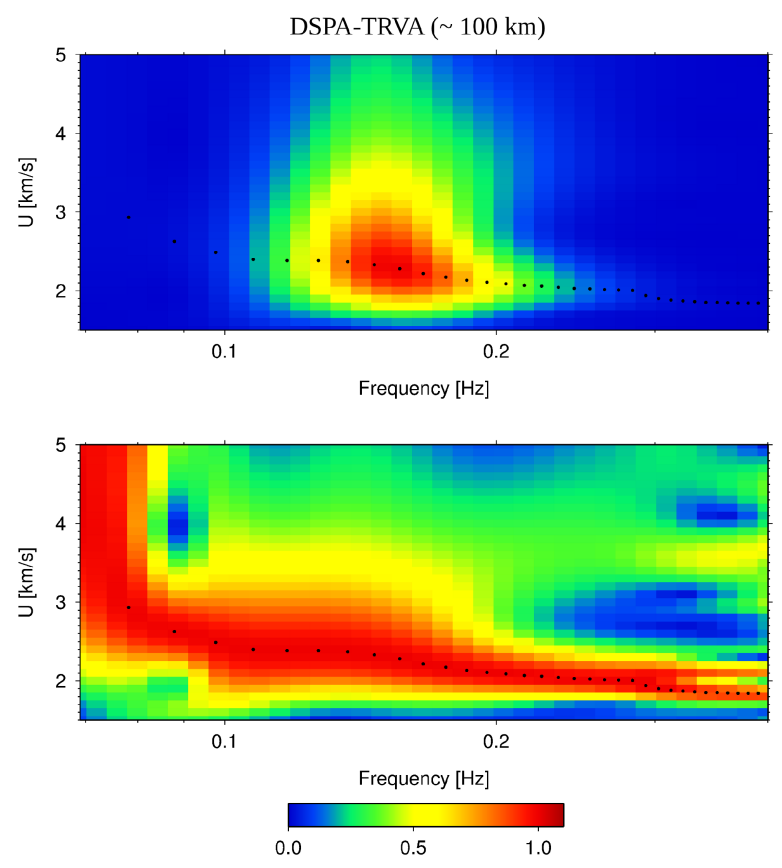

Figura 6.3: Velocidades de grupo obtenidas a partir de la correlación cruzada de los registros de ruido sísmico obtenido en DSPA-TRVA. Los puntos negros representan la velocidad de grupo en función de la frecuencia. Para el cálculo se utilizó el apilamiento lineal de la correlación cruzada (PCC) de un año de datos. El gráfico superior demuestra la distribución de energía normalizado a uno. En el gráfico inferior se normalizó la energía de cada frecuencia a uno para visualizar la llegada de grupo o energía. El eje de las frecuencias es logarítmico. La distancia entre las estaciones DSPA y TRVA es 100 km.

Este procedimiento se realizó para todas las correlaciones cruzadas que se obtuvieron en TdF. Las curvas de dispersión representadas en función de la frecuencia que no se enseñan aquí se muestran en el Apéndice B. En la Figura 6.4 se observan todas las curvas de dispersión en función del período, extraídas a partir de todas las correlaciones cruzadas realizadas considerando las cuatro estaciones de banda ancha de TdF. 

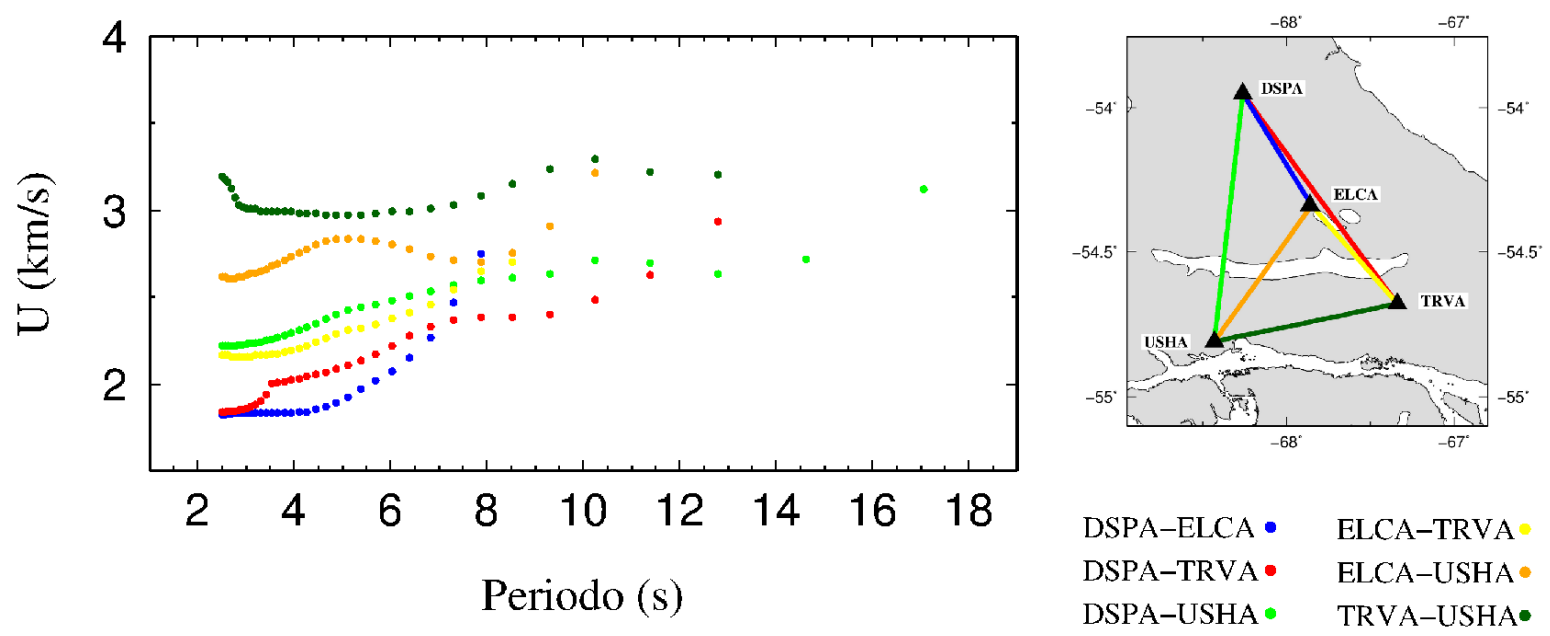

Figura 6.4: Izq.: Curvas de dispersión obtenidas a partir de las correlaciones cruzadas (PCC) para todas las combinaciones de estaciones posibles. Se consideró el apilamiento de un año de datos en todos los casos. Los diferentes colores indican las estaciones sismológicas involucradas en el cálculo de cada curva. Las velocidades de grupos fueron calculadas en los rangos de frecuencias que se muestran en la Tabla 6.1. Der.: Trayectorias entre estaciones, los colores se corresponden con las curvas de dispersión calculadas para cada par de estaciones.

\subsubsection{Efecto de la longitud del apilamiento y de la época estacional del año}

En la Figura 5.11 del Capítulo V, se observa el resultado de realizar el apilamiento de las correlaciones cruzadas de ruido sísmico considerando un año de datos, en particular los registros de todas las estaciones sismológicas durante el año 2008. Sin embargo, es interesante comprobar la robustez de los resultados analizando si diferentes longitudes de apilamiento de las correlaciones pueden indicar cambios en las curvas de dispersión determinadas.

En la Figura 6.5 se observan tres ejemplos del apilamiento secuencial de 10, 20 y 30 días consecutivos a partir de los registros de ruido sísmico obtenidos durante el verano y sus respectivas curvas de dispersión. En este caso, es apreciable la rápida convergencia del apilamiento de las correlaciones a la función empírica de Green y la estabilidad de las curvas de dispersión en general.

Por otro lado, se analizó además el apilamiento considerando diferentes épocas del año. La fuente de ruido sísmico es una fuente continua pero ésta puede variar dependiendo la época del año ya que el origen principal del ruido está relacionado con la actividad oceánica y ésta a su vez está influenciada por la actividad atmosférica que varía ampliamente desde el verano al invierno. McNamara y Buland (2004) analizaron los niveles de ruido ambiente en estaciones de banda ancha 
localizadas en Estados Unidos y encontraron que existen variaciones considerables en el ruido sísmico registrado durante el transcurso del día y entre las estaciones del año. Por otro lado, Stutzmann et al. (2009) demostraron que el espectro del microsismo secundario tiene ciertas características a nivel global que dependen de la latitud de la estación y de la época del año. En dicho estudio, observaron que la amplitud del ruido sísmico es mayor en ambos hemisferios durante el invierno, mientras que para latitudes cercanas al ecuador, la amplitud es estable durante todo el año. En el caso del área de estudio de esta Tesis, la localización de las estaciones se encuentra en una zona remota y el clima varía dependiendo de la época del año. Tal como se mencionara en el Capítulo I se reitera la dificultad de acceso que tienen algunas estaciones principalmente durante los meses de invierno y debido a esto, la escasez de datos en dichos meses.

En la Figura 6.6 se observan las curvas de dispersión obtenidas a partir de las correlaciones cruzadas entre tres pares de estaciones sismológicas, considerando el mes de enero (verano), junio (invierno) y noviembre (primavera). En el caso del resultado obtenido para DSPA-TRVA, las curvas de dispersión obtenidas muestran estabilidad para diferentes épocas del año. En el caso de TRVA-USHA, las curvas obtenidas considerando los registros del verano y el invierno muestran una diferencia para períodos cortos $(2.5 \mathrm{~s}<\mathrm{T}<3 \mathrm{~s})$. Los valores de velocidad de grupo encontradas son mayores para el invierno y menores durante el verano. Esta diferencia no fue observada en el caso de ELCA-TRVA. En este último caso, los valores de velocidad de grupo obtenidos son mayores durante la primavera y menores y prácticamente iguales durante el verano y el invierno (Figura 6.6) lo cual demuestra que las fuentes de ruido, a dichos períodos, varían según la época del año.

De esta manera, a partir de los datos obtenidos en las estaciones de TdF, se puede concluir que las fuentes de ruido sísmico en esta zona varían dependiendo la estación del año. Estos cambios de velocidades pueden deberse a fuentes de ruido localizadas en diferentes áreas, siendo interesante analizar en un futuro las direcciones de las fuentes principales de ruido en esta zona. De ambas figuras se puede concluir que las curvas de dispersión demuestran mayor variación si se analizan diferentes épocas del año y no tanto si se utilizan diferentes apilamientos para la misma época. En el primer caso, se obtiene rápidamente una convergencia a una forma de onda más estable y consecuentemente a una curva de dispersión, pero dichas curvas pueden estar sesgadas por la época del año debido a las particularidades o fenómenos meteorológicos y distribuciones de fuentes de ruido (Figura 6.6) que causan un campo ondulatorio, el cual es anisótropo con predominancia de ciertas direcciones de propagación que varía en función de la época del año. Esto demuestra la importancia de promediar los datos para un período de tiempo mayor. La línea punteada negra en la Figura 6.6 representa la media anual y se espera que se aproxime a la dispersión esperada. En este caso, las principales variaciones se observan para frecuencias bajas y altas (alrededor de $\mathrm{T}=3 \mathrm{~s}$ y $\mathrm{T}=8 \mathrm{~s}$ ) mientras que para los períodos intermedios ( $3 \mathrm{~s}<\mathrm{T}>8 \mathrm{~s}$ ) las curvas se mantienen más estables. 
a)

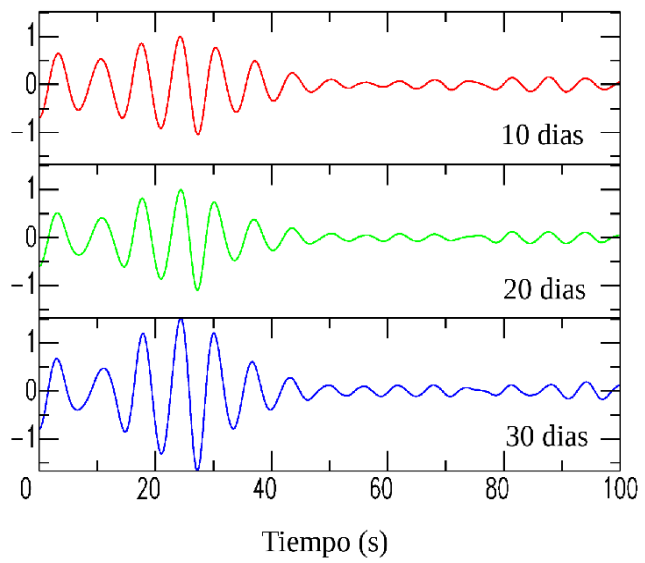

b)

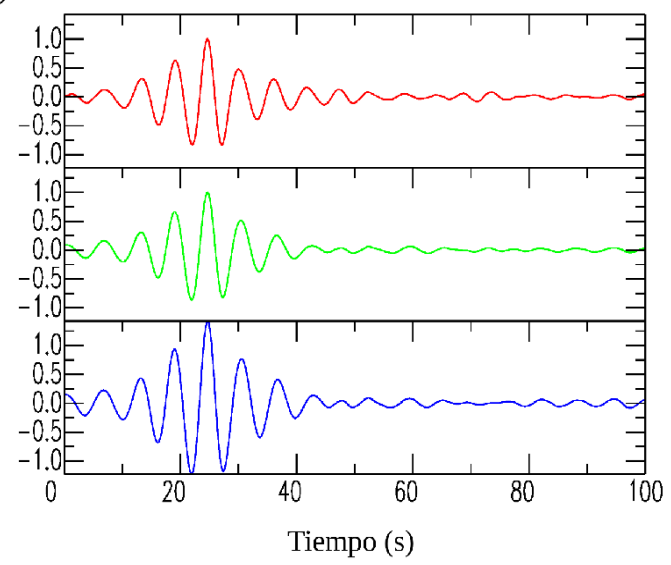

c)

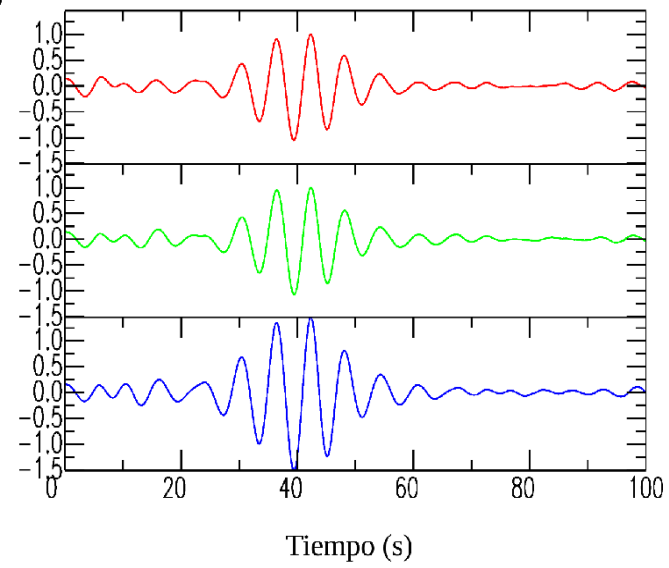

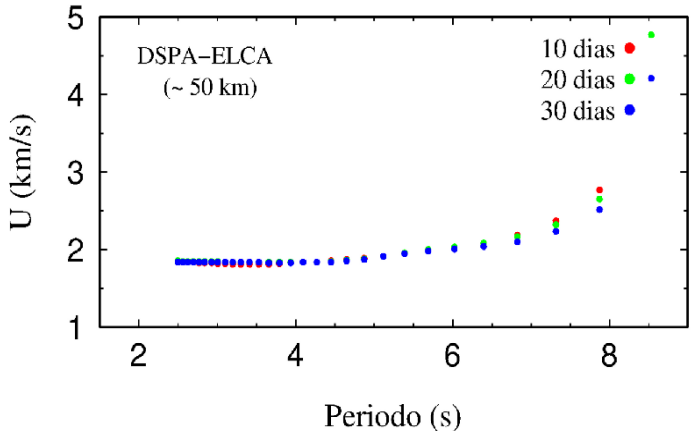
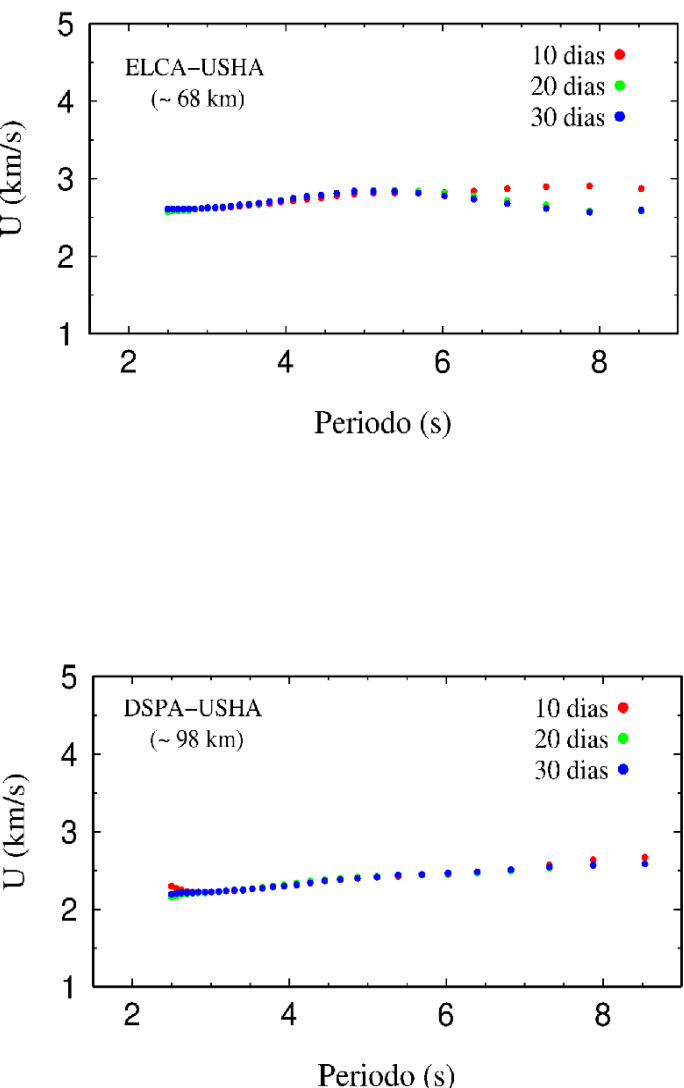

Figura 6.5: Apilamiento secuencial de 10, 20 y 30 días consecutivos de las correlaciones cruzadas (PCC) (izquierda) realizadas considerando a) DSPA-ELCA (50 km de distancia), b) ELCA-USHA (68 km de distancia) y c) DSPA-USHA (98 km de distancia). A la derecha se observa la curva de dispersión obtenida en cada caso. 

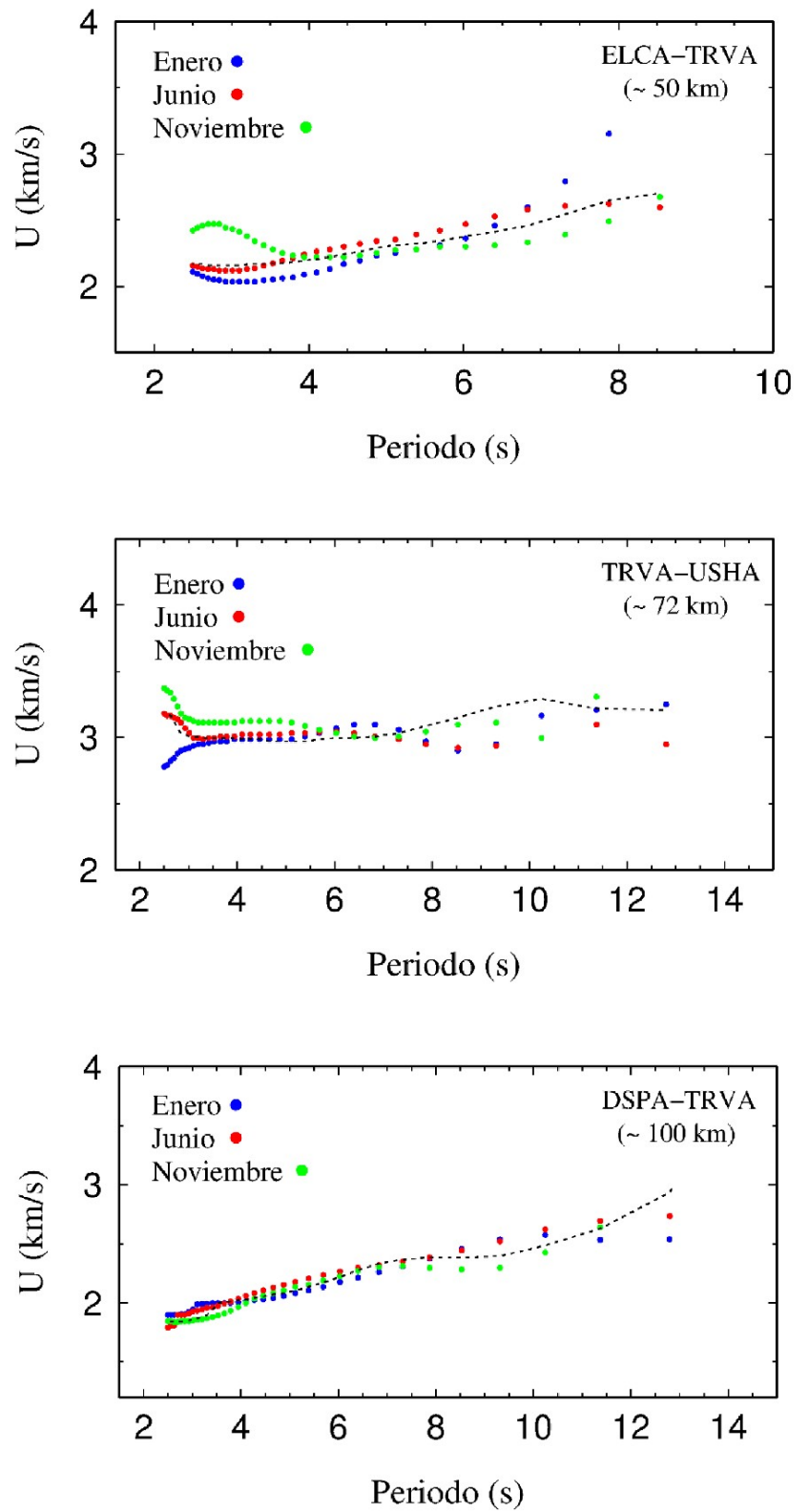

Figura 6.6: Curvas de dispersión obtenidas a partir de la correlación cruzada (PCC) entre a) DSPA y TRVA, b) TRVA y USHA y c) ELCA y TRVA, considerando tres meses diferentes del año. La línea punteada negra indica la curva de dispersión obtenida a partir de la correlación cruzada de los registros de ruido sísmico de un año. Se observa una pequeña divergencia entre las curvas para los períodos 2s y 4s en los dos paneles superiores lo cual podría deberese al efecto de fuentes de ruido locales.

\subsection{Inversión de las velocidades de grupo}

Las velocidades de dispersión de las ondas superficiales están relacionadas con las velocidades de la onda $\mathrm{P}$, “ $\mathrm{V}_{\mathrm{P}}$ ”, de la onda $\mathrm{S}$, "V $\mathrm{V}_{\mathrm{S}}$ ", con la densidad del medio " $\rho$ ” y los espesores de las 
diversas capas "e":

$$
c, u=f\left(V_{p}, V_{s}, \rho, e\right)
$$

siendo c y u las velocidades de fase y grupo de las ondas superficiales, respectivamente.

Es decir, a partir de la inversión de las velocidades de ondas superficiales es posible obtener una estructura de velocidades de ondas sísmicas del subsuelo. Dicha relación es no-lineal y los valores de $V_{P}, V_{S}$ y $\rho$ calculados a partir de curvas de dispersión no son únicos. En general, las velocidades de las ondas superficiales son principalmente sensibles a la velocidad de onda $\mathrm{S}$ y por este motivo, normalmente se calcula sólo $\mathrm{V}_{\mathrm{s}}$.

Para determinar la estructura de velocidad de onda $\mathrm{S}$ se utilizan métodos de inversión que buscan el modelo que mejor ajusta a los valores observados de las ondas superficiales. El proceso consiste en generar velocidades de dispersión para diferentes modelos de $\mathrm{V}_{\mathrm{S}}$ que se comparan con las observadas y se realiza un proceso iterativo en el que se ajustan los parámetros del modelo para minimizar la diferencia entre las velocidades observadas y calculadas. La solución no es única y generalmente se consigue una familia de modelos que se ajustan a los datos observados.

Para realizar la inversión de las curvas de dispersión en este trabajo, se aplicó el código SURF96 que está incluido dentro del paquete Computer Programs of Seismology (Herrmann, 2013). Dicho algoritmo realiza un proceso iterativo de inversión a partir de un modelo inicial y además ofrece la posibilidad de asignar pesos a las distintas capas.

Cuando se quiere estimar la velocidad de onda S mediante una inversión basada en la inversión por mínimos cuadrados, como es el caso de SURF96, el modelo inicial tiene una importante influencia sobre los resultados. Para obtener modelos confiables, es importante partir de alguna información previa conocida para la zona de estudio o iniciar la inversión utilizando varios modelos iniciales para encontrar las diferentes familias de modelos que puedan explicar las observaciones. A tal efecto, para definir los modelos iniciales se consideró como punto de partida el modelo CRUST 1.0 (Laske et al., 2013) definido para la zona de estudio (Tabla 6.2).

\begin{tabular}{cccc}
\hline \hline Espesor $(\mathrm{km})$ & $\mathrm{V}_{\mathrm{P}}(\mathrm{km} / \mathrm{s})$ & $\mathrm{V}_{\mathrm{S}}(\mathrm{km} / \mathrm{s})$ & $\rho\left(\mathrm{g} / \mathrm{cm}^{3}\right)$ \\
\hline \hline 1 & 2.50 & 1.07 & 2.11 \\
2.5 & 4.00 & 2.13 & 2.37 \\
8 & 6.00 & 3.50 & 2.72 \\
10.5 & 6.50 & 3.74 & 2.82 \\
10 & 7.10 & 4.04 & 2.99 \\
\hline
\end{tabular}

Tabla 6.2: Modelo CRUST 1.0 correspondiente a la zona de estudio (Laske et al., 2013). 
Cuando se realiza la inversión considerando un modelo inicial con velocidades bajas, como es el caso del CRUST 1.0 para algunas regiones de la Tierra, es probable que el resultado converja a modelos con zonas de baja velocidad (comunicación personal de R. Herrmann). Esto fue comprobado mediante las primeras pruebas que se realizaron con el fin de definir el modelo inicial a utilizar y otros parámetros de la inversión.

Por consiguiente para realizar la inversión de las 6 curvas de dispersión se consideraron tres modelos iniciales. Los primeros dos modelos iniciales A y B (Tabla 6.3) son una parametrización adaptada a partir del modelo CRUST 1.0 obtenido para TdF. En ambos casos se dividió los primeros $10 \mathrm{~km}$ de corteza en capas de $1 \mathrm{~km}$ de espesor y se consideró un leve aumento del valor de las velocidades sísmicas. Esta discretización en capas se llevó a cabo con el fin de considerar ciertas incertidumbres en profundidad y teniendo en cuenta que el dato obtenido de las curvas de dispersión en este trabajo es sensible a los primeros km de profundidad de corteza.

El tercer modelo inicial C, es el modelo final obtenido a partir de realizar la inversión considerando al modelo B como inicial. De esta manera, el modelo C es diferente para cada curva de dispersión. Los valores de velocidad y densidad considerados para el manto en los tres modelos iniciales fueron: $V_{\mathrm{P}}=7.99 \mathrm{~km} / \mathrm{s}, \mathrm{V}_{\mathrm{S}}=4.44 \mathrm{~km} / \mathrm{s}$ y $\rho=3.3 \mathrm{~g} / \mathrm{cm}^{3}$, según el modelo CRUST 1.0.

\begin{tabular}{cccc}
\hline \hline Espesor $(\mathrm{km})$ & $\mathrm{V}_{\mathrm{P}}(\mathrm{km} / \mathrm{s})$ & $\mathrm{V}_{\mathrm{S}}(\mathrm{km} / \mathrm{s})$ & $\rho\left(\mathrm{g} / \mathrm{cm}^{3}\right)$ \\
\hline \hline 1 & $3.50(3.80)$ & $1.52(1.63)$ & 2.1 \\
1 & $3.80(4.00)$ & $1.63(1.71)$ & 2.2 \\
1 & $4.20(4.30)$ & $2.24(2.30)$ & 2.3 \\
1 & $4.70(4.80)$ & $2.51(2.56)$ & 2.4 \\
1 & $4.90(5.00)$ & $2.62(2.70)$ & 2.5 \\
1 & $5.20(5.30)$ & $2.88(2.94)$ & 2.6 \\
1 & $5.50(5.60)$ & $3.07(3.11)$ & 2.7 \\
1 & $5.80(5.90)$ & $3.31(3.40)$ & 2.7 \\
1 & $5.90(6.10)$ & $3.47(3.50)$ & 2.7 \\
1 & $6.00(6.20)$ & $3.50(3.64)$ & 2.8 \\
10.5 & $6.50(6.60)$ & $3.74(3.78)$ & 2.9 \\
10 & $7.10(7.10)$ & $4.04(4.04)$ & 2.9 \\
\hline
\end{tabular}

Tabla 6.3: Modelos iniciales A y B. Los valores de las velocidades entre paréntesis corresponden al Modelo B.

Se realizaron varias pruebas preliminares con el fin de establecer los valores de los parámetros de la inversión, suavizados y factores de pesos. Considerando que la inversión se centró en los primeros $10 \mathrm{~km}$ de corteza, se asignaron pesos a las últimas capas de manera que sus valores 
permanezcan constantes. Además se analizó la inversión primero fijando los espesores e invirtiendo la velocidad y viceversa. Finalmente se llevaron a cabo tres inversiones (entre 50 y 200 iteraciones) para cada curva de dispersión considerando los tres modelos iniciales.

\subsubsection{Resultados}

En las Figuras 6.7 y 6.8 se observa el resultado de la inversión de cada curva de dispersión considerando los tres modelos iniciales A, B y C. Por otro lado, se muestra como resultado final, los valores del error estándar obtenido en cada caso (Tabla 6.4).

\begin{tabular}{ccc}
\hline \hline Estaciones & Modelo inicial & Error estándar $(\mathbf{k m} / \mathbf{s})$ \\
\hline \hline ELCA-TRVA & Modelo A & 0.0180 \\
$(50 \mathrm{~km})$ & Modelo B & 0.0161 \\
& Modelo C & 0.0153 \\
\hline \hline DSPA-ELCA & Modelo A & 0.0415 \\
$(50 \mathrm{~km})$ & Modelo B & 0.0420 \\
& Modelo C & 0.0406 \\
\hline \hline DSPA-TRVA & Modelo A & 0.0308 \\
$(100 \mathrm{~km})$ & Modelo B & 0.0333 \\
& Modelo C & 0.0306 \\
\hline \hline DSPA-USHA & Modelo A & 0.0168 \\
$(98 \mathrm{~km})$ & Modelo B & 0.0159 \\
& Modelo C & 0.0095 \\
\hline \hline TRVA-USHA & Modelo A & 0.0369 \\
$(72 \mathrm{~km})$ & Modelo B & 0.0376 \\
& Modelo C & 0.0383 \\
\hline \hline ELCA-USHA & Modelo A & 0.0464 \\
$(68 \mathrm{~km})$ & Modelo B & 0.0467 \\
& Modelo C & 0.0491 \\
\hline \hline
\end{tabular}

Tabla 6.4 Valores del error estándar de velocidad obtenido en la inversión de las curvas de dispersión. La primer columna de la tabla indica el par de estaciones sismológicas de banda ancha consideradas para la estimación de la correlación cruzada (PCC) o trayectoria de las ondas superficiales. El valor debajo indicado entre paréntesis de cada par de estaciones representa la distancia horizontal entre las mismas. 
a)

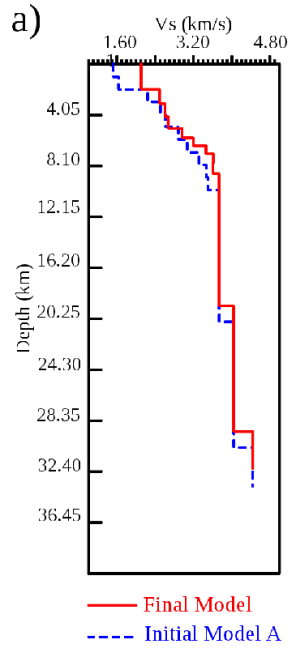

b)

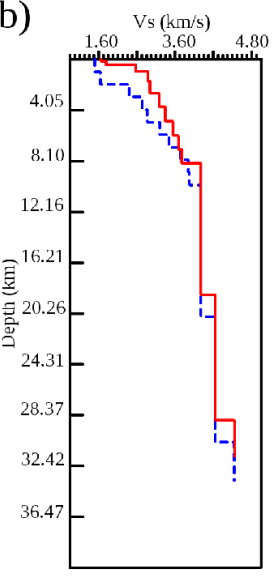

_ Final Model ---- Initial Model A

c)

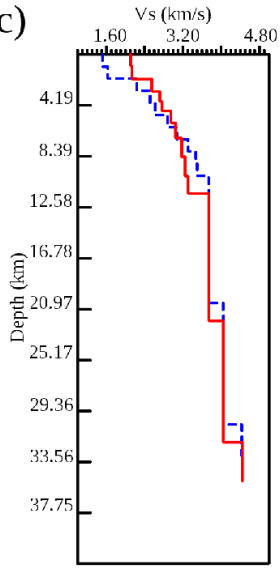

__-_- Initial Model A
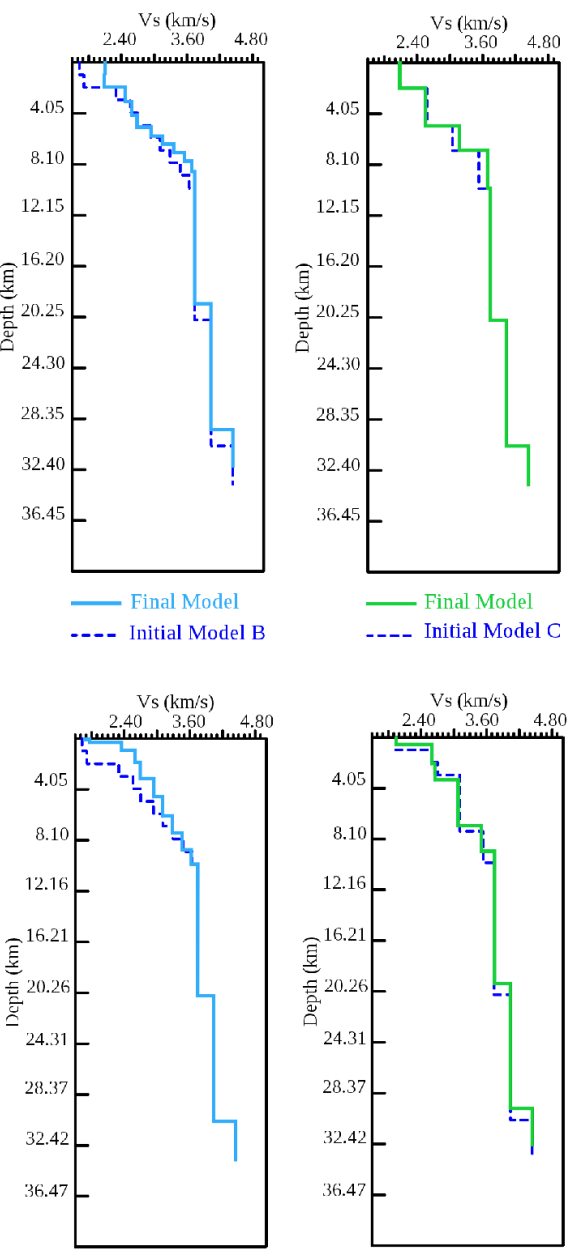

_.-- Initial Model B
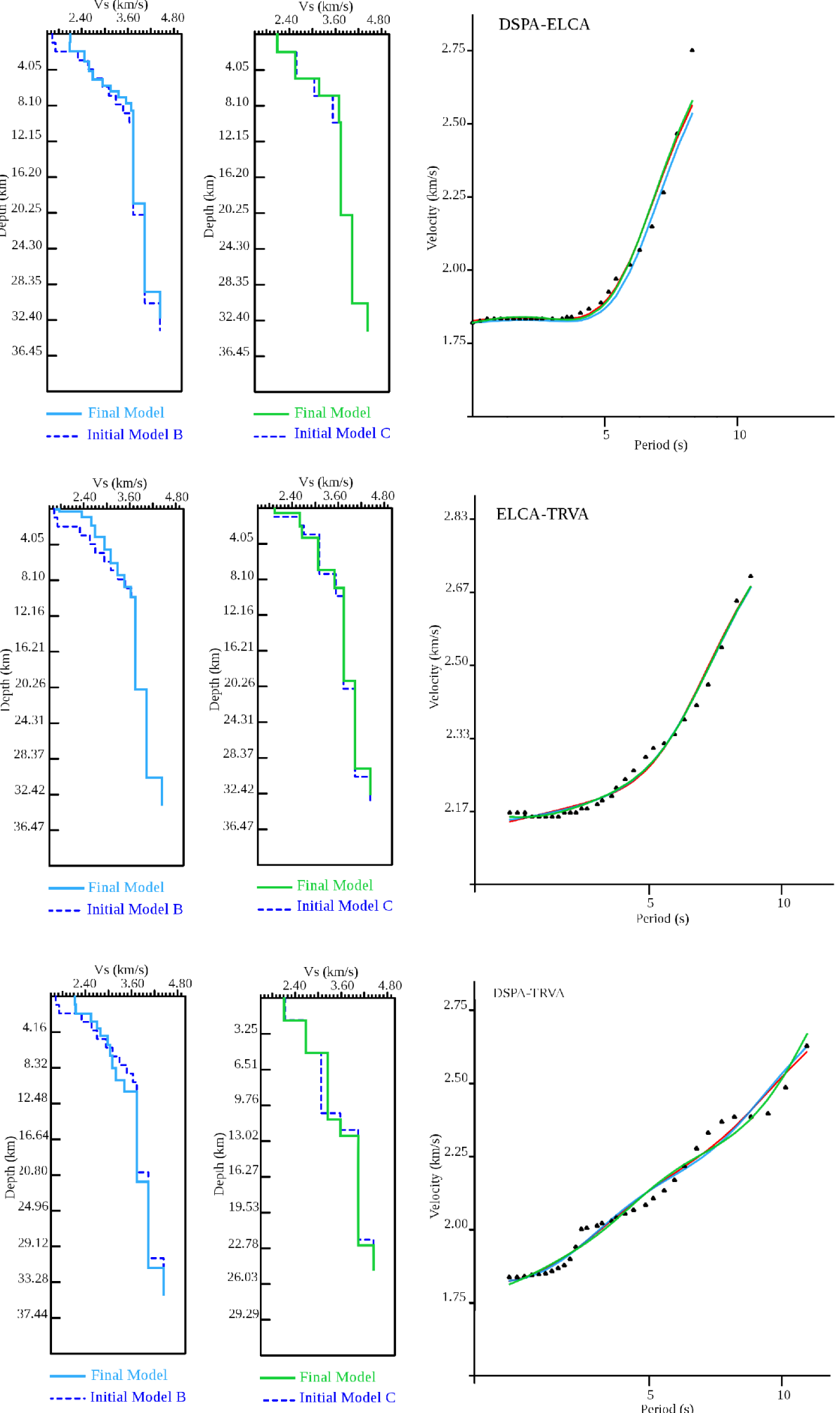

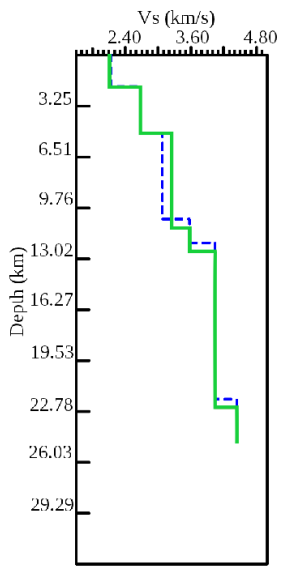

_._. Finitial Model C

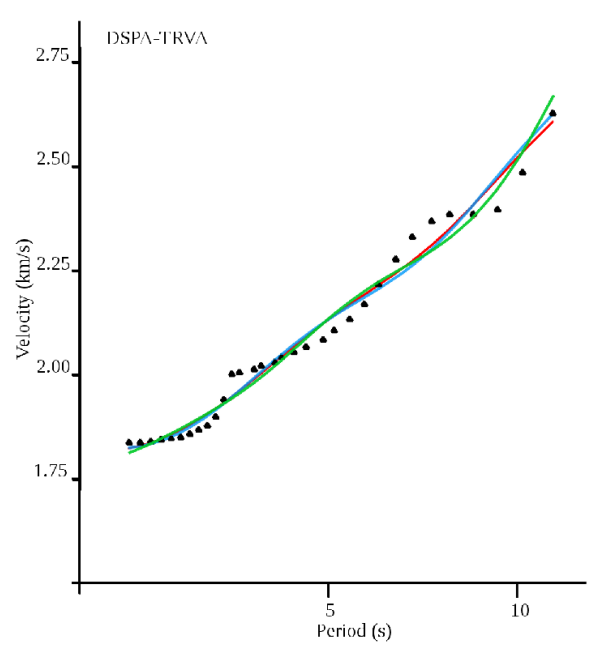

Figura 6.7: Resultado de la inversión de la curva de dispersión obtenida en el trayecto a) DSPA-ELCA, b) ELCA-TRVA y c) DSPA-TRVA. Los modelos finales en rojo, azul y verde se corresponden con los modelos iniciales A, B y C, repectivamente. A la derecha se observan las velocidades de grupo observadas (triángulos negros) y calculadas para el modelo que mejor ajusta (líneas roja, azul y verde). 


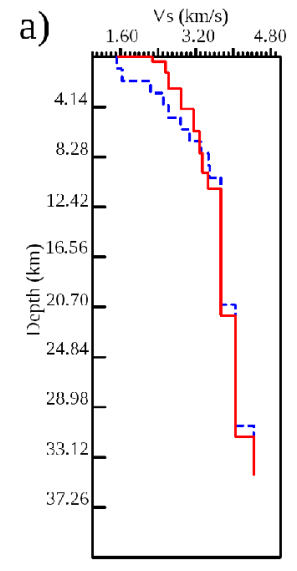

_ Tinal Model ..... Initial Model A

b)

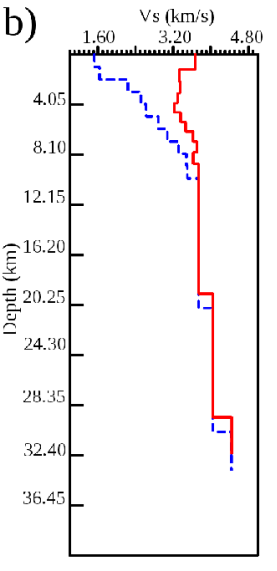

__-_ Initial Model A

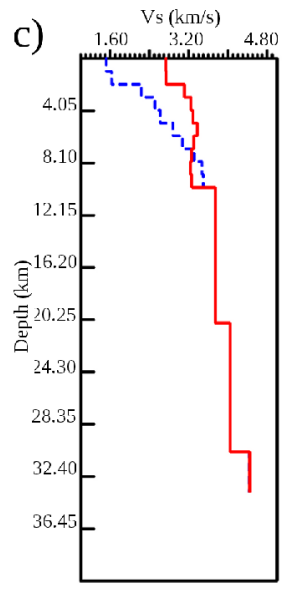

_-_-_ Initial Model $A$

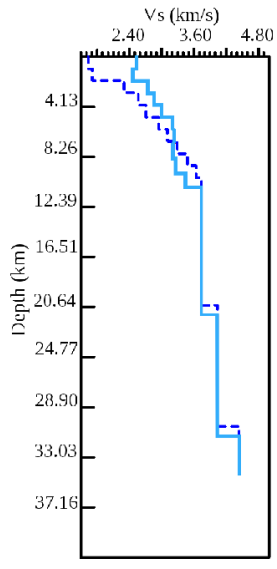

- Tinal Model _-_. Initial Model B

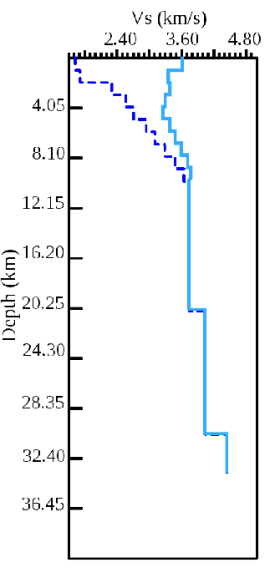

_.--_ Initial Model B

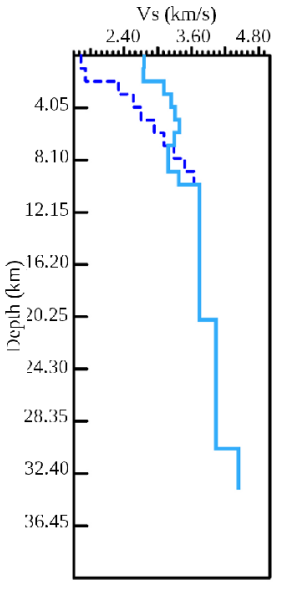

-_-_ Initial Model P

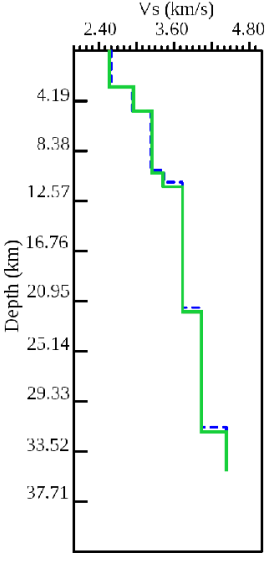

_._-_. Initial Model C

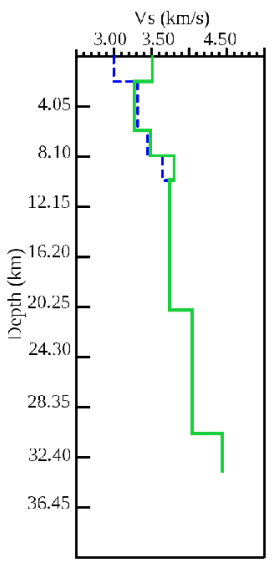

_- Final Model

$\mathrm{Vs}(\mathrm{km} / \mathrm{s})$

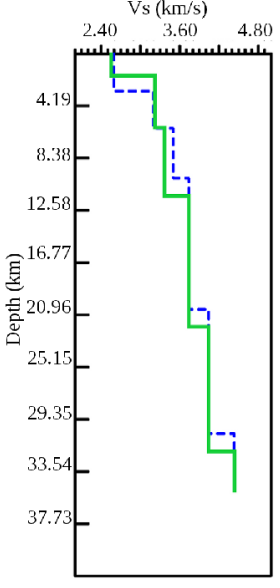

_ rinal Model
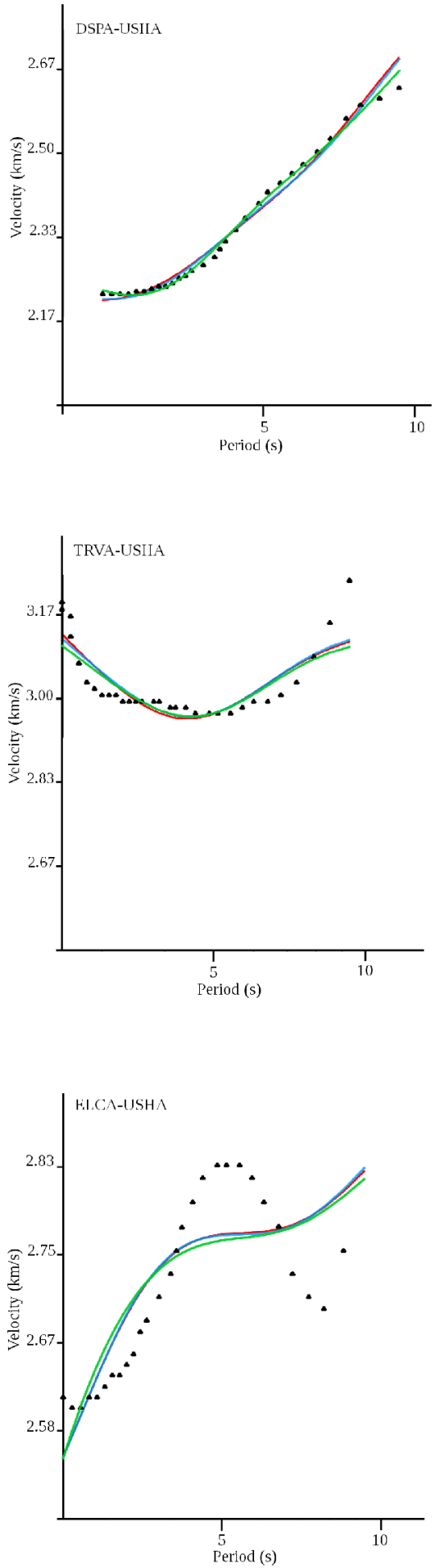

Figura 6.8: Resultado de la inversión de la curva de dispersión obtenida en el trayecto a) DSPA-USHA, b) TRVA-USHA y c) ELCA-USHA. Los modelos finales en rojo, azul y verde se corresponden con los modelos iniciales A, B y C, repectivamente. A la derecha se observan las velocidades de grupo observadas (triángulos negros) y calculadas para el modelo que mejor ajusta (líneas roja, azul y verde). 


\subsubsection{Discusión y conclusiones}

Los modelos finales obtenidos a partir de la inversión de la curvas de dispersión ajustan bien a las velocidades de grupo observadas en el caso de las trayectorias de ondas superficiales obtenidas para ELCA-TRVA, DSPA-ELCA, DSPA-TRVA y DSPA-USHA. Por el contrario, el ajuste obtenido no fue tan bueno cuando se realizó la inversión de las curvas obtenidas para los trayectos TRVAUSHA y ELCA-USHA. Estas últimas dos trayectorias corresponden a las zonas de la Isla donde se obtuvieron las velocidades de grupo más altas en la zona central y sur de la zona de estudio.

La variabilidad de los modelos obtenidos en este capítulo se correlaciona con el diverso ambiente geológico y tectónico presente en la Isla, a través de la presencia de fallas y estructuras plegadas (Olivero y Martinioni, 2001, Kraemer, 2003; Lodolo et al., 2003, 2007; Tassone et al., 2005; Menichetti et al., 2008). A pesar de esto, se distinguen algunas discontinuidades que coinciden en profundidad en varios de los modelos finales obtenidos a partir de la inversión de las curvas de dispersión (a 2, 5 y $7 \mathrm{~km}$ de profundidad aproximadamente). Estas discontinuidades podrían estar indicando cambios de geología y litología en los primeros km de corteza.

La mayor complejidad se observa en los modelos obtenidos para los trayectos TRVA-USHA y ELCA-USHA, para los cuales el ajuste no fue tan bueno y en los cuales los modelos revelan zonas de baja velocidad entre $1.5 \mathrm{~km}-4 \mathrm{~km}$ de profundidad, en el primer caso, y $5 \mathrm{~km}-9 \mathrm{~km}$ de profundidad en el segundo caso, aproximadamente. Además para estos dos casos, los resultados obtenidos están relacionados con una estructura media de velocidad de onda S caracterizada por valores altos (2.5-3.1 km/s) en los primeros kilómetros de corteza. En particular, los modelos obtenidos para el trayecto TRVA-USHA, muestran velocidades $\mathrm{V}_{\mathrm{S}}=3.6 \mathrm{~km} / \mathrm{s}$ en los primeros kilómetros. Esto parece estar relacionado con la presencia de cuerpos magmáticos intrusivos y la ausencia de sedimentos en ciertas áreas de la zona sur de la Isla cercanas a las estaciones TRVA y USHA (Adaros Cárcamo, 2003; Lawrence y Wiens, 2004; Tassone et al., 2005). 


\section{CAPÍTULO VII}

\section{Resultados y Conclusiones}

En este trabajo se realiza una estimación de la estructura cortical de TdF utilizando datos de cuatro estaciones sismológicas de banda ancha instaladas en la Isla. Esta Tesis representa el primer estudio realizado en TdF a partir de datos de una red local de estaciones sismológicas.

En primera instancia se determinaron espesores corticales, relación de velocidades sísmicas $\mathrm{V}_{\mathrm{P}} / \mathrm{V}_{\mathrm{S}}$ y modelos de velocidad de onda $\mathrm{S}$ a partir del cálculo, apilamiento e inversión de FRs desarrollado en los Capítulos II, III y IV. Se implementaron dos técnicas: el método de H-k stacking y la inversión de FRs mediante el algoritmo de Neighbourhood Algorithm (NA). El análisis de los datos se separó en dos grupos: telesismos provenientes del SO (grupo 1 y de color azul) y eventos provenientes del $\mathrm{N}$ (grupo 2 y de color rojo, como se muestra en la Figura 7.1) de TdF. Esto permitió analizar con mayor detalle los valores obtenidos en cada caso y detectar cambios en la estructura incluso en los alrededores de una misma estación. En la Figura 7.1 se muestran los valores de espesor cortical y de relación de velocidades sísmicas obtenidos con el método de H-k stacking y con la inversión de FRs con el algoritmo NA. Los valores de espesor cortical y de la relación de velocidades sísmicas $\mathrm{V}_{\mathrm{P}} / \mathrm{V}_{\mathrm{S}}$ obtenidos a partir del primer método varían en el rango 24.8-34.8 km y 1.56-1.80, respectivamente, dependiendo de la estación y del grupo de eventos utilizados. En cuanto a la segunda técnica, el espesor cortical estimado varía desde 24.5 a $39.2 \mathrm{~km}$ y la relación $\mathrm{V}_{\mathrm{P}} / \mathrm{V}_{\mathrm{S}}$ se estimó en el rango 1.69-1.88. De esta manera, la técnica NA sugiere mayor espesor cortical y valores más altos de la relación de velocidades sísmicas en ciertas zonas de la Isla. Estas variaciones de la estructura observadas se correlacionan con la presencia de diferentes ambientes geológicos, sistemas de fallas y estructuras plegadas presentes en la Isla (Olivero y Martinioni, 2001; Kraemer, 2003; Lodolo et al., 2003, 2007; Tassone et al., 2005; Menichetti et al., 2008; Peroni et al., 2009; Torres-Carbonell y Dimieri, 2013). Los valores de espesor cortical obtenidos en la mayoría de las estaciones sismológicas, mediante las dos técnicas, indican que la profundidad de la Moho en el sector oriental de TdF aumenta de norte a sur. Además, los valores de espesor cortical determinados con ambas técnicas, son similares a valores obtenidos en estudios previos realizados mediante otras metodologías en zonas cercanas al área de estudio de este trabajo (Ludwig et al., 1965; Robertson Maurice et al., 2003; Lawrence y Wiens, 2004). 

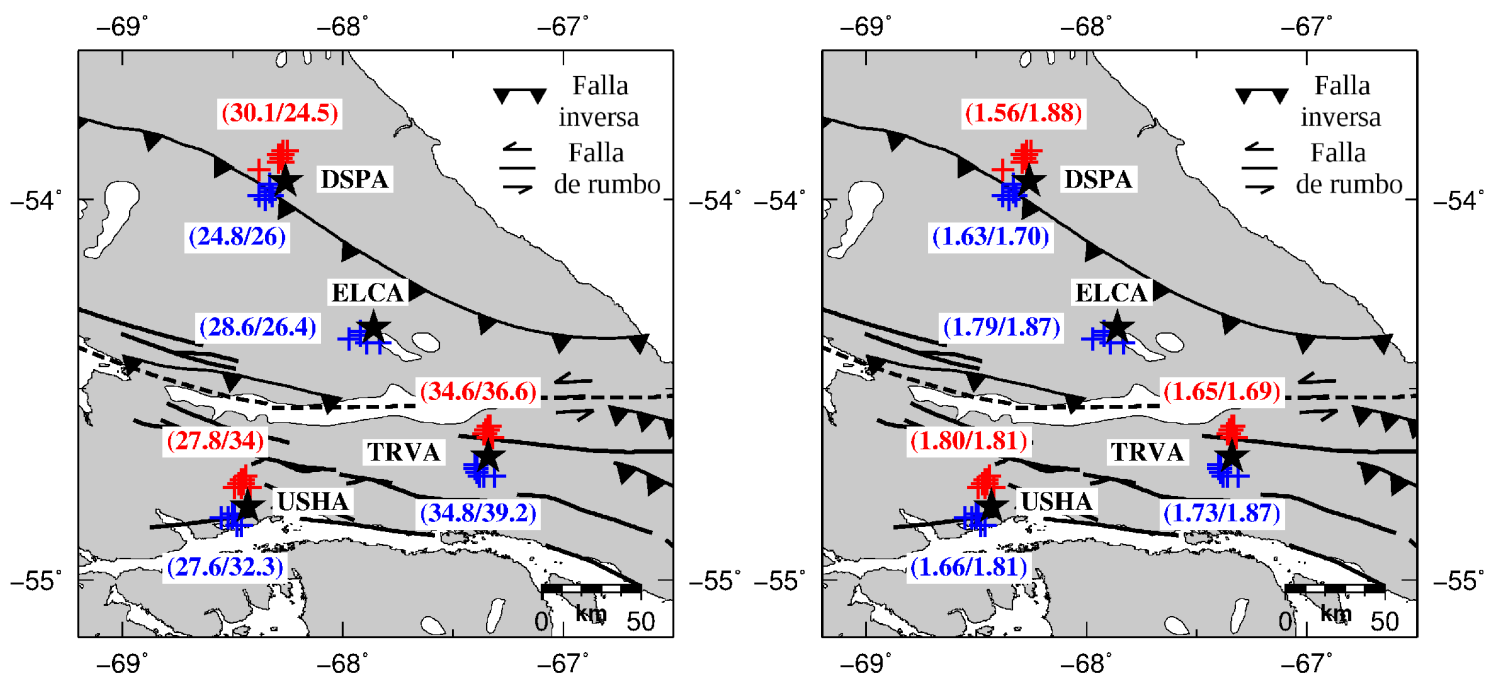

Figura 7.1: Valores de espesor cortical (izquierda) y de relación de velocidades sísmicas $\mathrm{V}_{\mathrm{P}} / \mathrm{V}_{\mathrm{S}}$ (derecha), obtenidos mediante el método H-k stacking (valor a la izquierda dentro del paréntesis) y

la inversión de FRs con el algoritmo NA (valor a la derecha dentro del paréntesis). En el caso del H-k stacking, el valor que se observa en los mapas es el promedio de las ocho soluciones obtenidas en cada grupo

(más información en el Capítulo III). Las cruces indican los puntos de conversión de las fases para los eventos seleccionados considerando una profundidad hipotética de $30 \mathrm{~km}$ (en azul para el grupo SO y en rojo para el grupo N). Las estrellas negras indican la ubicación geoǵrafica de las estaciones sismológicas. Las líneas continuas indican lineamientos y fallas geológicas. La línea punteada indica la FMF.

Para la inversión de las FRs realizada mediante el algoritmo NA se estima la incertidumbre utilizando la variación de la profundidad de la Moho para todos los modelos que tienen un desajuste menor al valor mínimo más el 10\%. El valor de 10\% es empírico y fue considerado para permitir una variación de los mejores modelos que ajustan bien a los datos. Como incertidumbre se utiliza dos veces la desviación estándar ya que este valor representa un $95.44 \%$ de todos los modelos considerando una distribución Gaussiana. Los valores de incertidumbre obtenidos para la profundidad de la Moho varían entre $0.7 \mathrm{~km}$ y $2 \mathrm{~km}$, mientras que en el caso de la relación de velocidades sísmicas, la incertidumbre está en el rango 0.01-0.029. Los mayores y menores errores fueron encontrados para los eventos provenientes del SO (grupo 1) de las estaciones ELCA y TRVA, respectivamente.

En cuanto a los valores de espesor cortical y la relación $V_{\mathrm{P}} / \mathrm{V}_{\mathrm{S}}$ obtenidos con la técnica de H-k stacking, la incertidumbre estimada se basa en el método de bootstrap (más detalle en la sección 3.2.1.2 del Capítulo III). Los valores de incertidumbre del espesor cortical varían desde $0.8 \mathrm{~km}$ a 3.8 km, siendo DSPA (grupo 2, N) y ELCA (grupo 1, SO) las estaciones donde se encontraron los 
valores mínimos y máximos, respectivamente. Con respecto a la relación $V_{\mathrm{P}} / \mathrm{V}_{\mathrm{s}}$, la incertidumbre está en el rango 0.05-0.2. El mínimo valor de incertidumbre (0.05) se obtuvo en la estación TRVA (grupo 1, SO) mientras que el máximo valor (0.2) se encontró en la estación USHA (grupo 2, N).

El error o incertidumbre exacto de los valores de espesor cortical y de $V_{\mathrm{P}} / \mathrm{V}_{\mathrm{S}}$ no puede ser determinado unívocamente por falta de control de todos las posibles fuentes de error. Los valores presentados aquí son aproximaciones y sirven para realizar comparaciones relativas dentro de cada metodología utilizada.

Por otro lado se implementó la técnica de interferometría sísmica desarrollada en el Capítulo V. Mediante dicho método, se extrajo información de ondas superficiales para ondas Rayleigh a través del cálculo de correlaciones cruzadas de trazas de ruido sísmico ambiental registradas en las cuatro estaciones sismológicas de banda ancha, obteniendo seis trayectos de ondas superficiales. En particular, se realizó el cálculo e inversión de velocidades de grupo del modo fundamental de ondas Rayleigh (Capítulo VI). El ajuste de la inversión de las curvas de dispersión obtenidas en los trayectos TRVA-USHA y ELCA-USHA no fue tan bueno como en el resto de los casos. Ambas trayectorias corresponden a las zonas de la Isla donde se obtuvieron las velocidades de grupo más altas ( 2.6 - $3.3 \mathrm{~km} / \mathrm{s}$ para períodos entre $3 \mathrm{~s}$ y 9 s) en la zona sur del área de estudio (Figura 7.2).
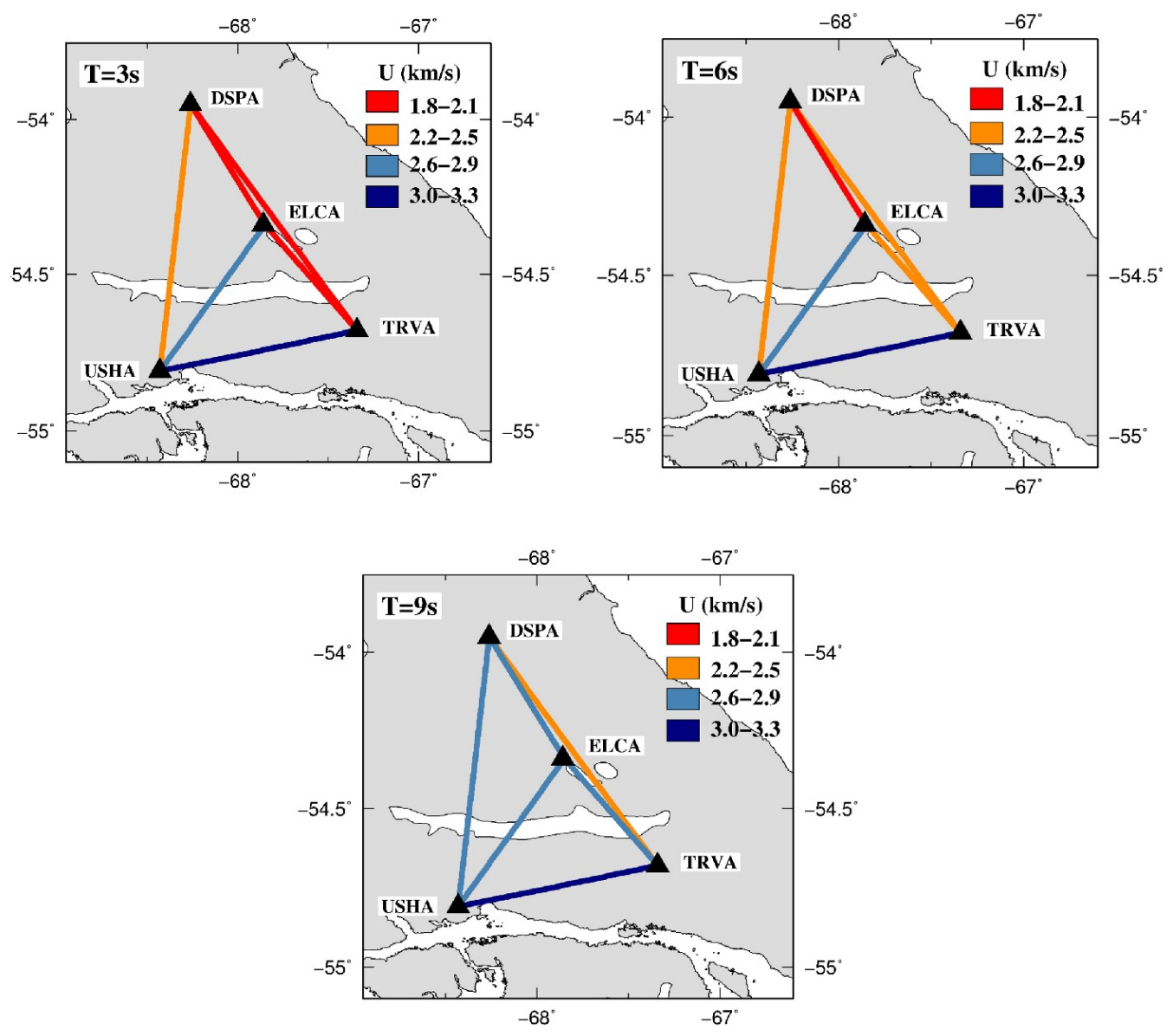

Figura 7.2: Velocidades de grupo obtenidas a partir de la correlación cruzada (PCC) de ruido sísmico 
ambiental registrado durante un año en las estaciones sismológicas de TdF (indicadas con triángulos negros). Cada línea representa un trayecto de ondas superficiales. Los colores de cada trayecto indican los valores de las velocidades obtenidas para los períodos 3, 6 y 9 s según la escala que se indica.

La variación que se observa entre los valores obtenidos para el trayecto DSPA-TRVA en comparación de DSPA-ELCA + ELCA-TRVA para T=9 s no es la esperada por la proximidad de los correspondientes trayectos entre estaciones y tal vez refleje algún tipo de camino múltiple (multipathing) para dicho período.

A partir de la inversión de las seis curvas de dispersión se estimaron modelos de velocidad de onda S que complementan la información de la estructura cortical obtenida a partir del método de FRs (Figura 7.3). Debido a la poca distancia entre estaciones (y por lo tanto altas frecuencias), dichos modelos aportan información de las capas más superficiales de la corteza. En particular, la inversión fue resuelta considerando los primeros $10 \mathrm{~km}$ de corteza.

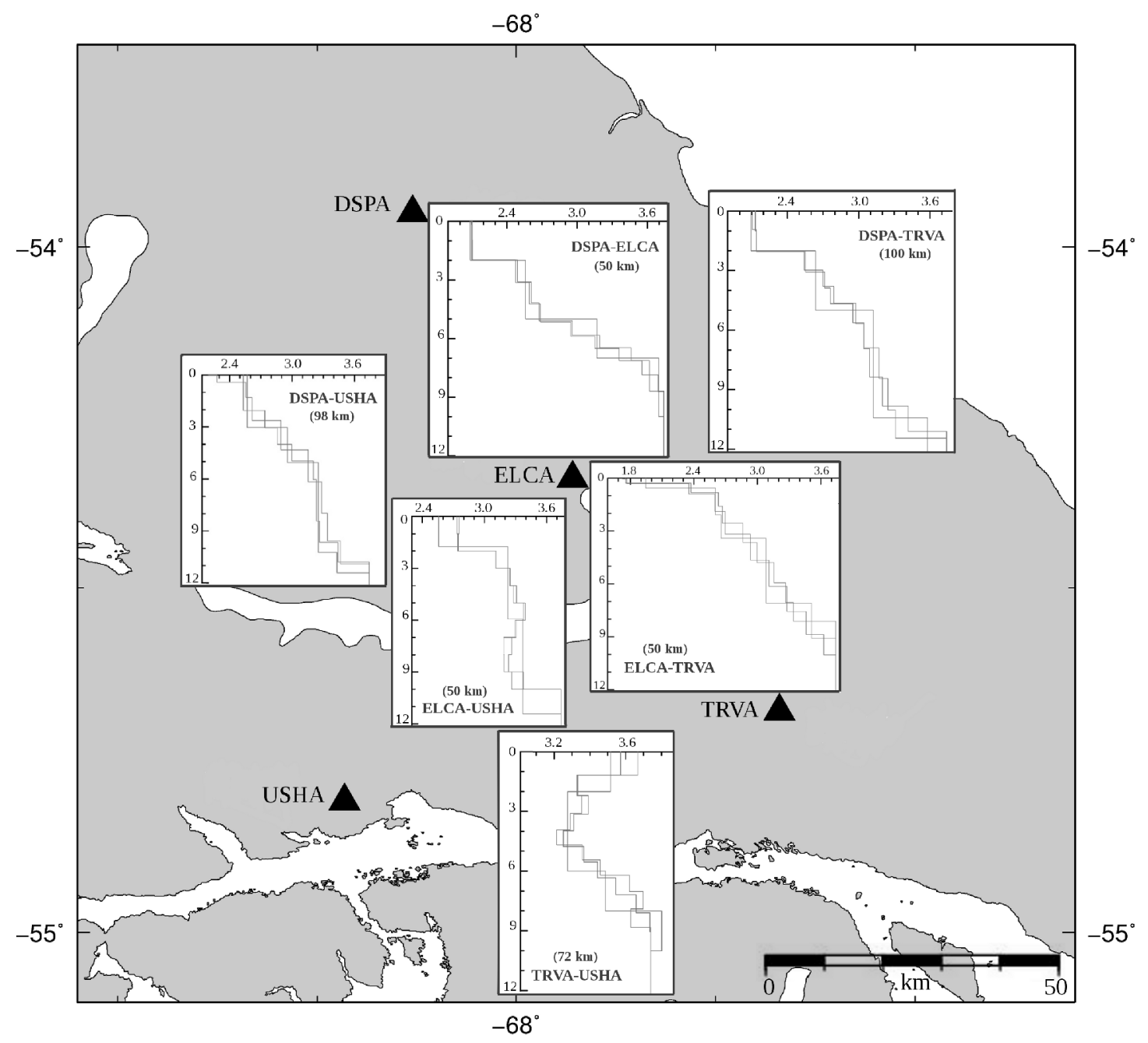

Figura 7.3: Resultado de los modelos de velocidad de onda S obtenidos a partir de la inversión de las curvas de dispersión obtenida de las correlaciones cruzadas entre cada par de estaciones (triángulos negros). En cada 
caso, se observan los 3 modelos finales que mejor ajustan a la estructura media de cada trayecto (el eje horizontal representa los valores de $\mathrm{V}_{\mathrm{S}}(\mathrm{km} / \mathrm{s})$ y el eje vertical la profundidad en $\left.\mathrm{km}\right)$. Además se indica en cada gráfico las estaciones involucradas en el cálculo.

La estructura superficial y local obtenida en la estación DSPA a partir de la inversión de FRs está caracterizada por velocidades de onda $S$ en el rango 1.75-1.80 km/s en los primeros $2 \mathrm{~km}$ de corteza. La estructura media obtenida a partir de la inversión de las curvas de dispersión calculada en los trayectos DSPA-TRVA, DSPA-ELCA y DSPA-USHA, presenta valores de $\mathrm{V}_{\mathrm{S}}$ entre 2.3 y 2.6 $\mathrm{km} / \mathrm{s}$ para las mismas profundidades. Por otro lado, la estación ELCA, ubicada en el centro de la zona de estudio, refleja una estructura local de onda S con valores de $2.4 \mathrm{~km} / \mathrm{s}$ en las capas más superficiales. Estos valores de velocidad de onda S obtenidos a partir de la estructura local y media entre las estaciones mencionadas son acordes con el ambiente geológico de la zona caracterizado por la presencia de sedimentos relacionados con los depósitos del Terciario y Cretácico superior presentes en la zona (Olivero y Martinioni, 2001; Tassone et al., 2005).

Las estaciones ubicadas en la zona sur (TRVA y USHA), demuestran que la estructura cortical en esa zona está caracterizada por una velocidad de onda S más alta en los primeros km de corteza respecto a las otras dos estaciones. Ambas estaciones se encuentran ubicadas sobre lo que se conoce como segmento de faja plegada y corrida interna, involucrando casi exclusivamente en la deformación y engrosamiento cortical, a las unidades del Cretácico inferior, Jurásico superior y al basamento del Paleozoico (Menichetti et al., 2008). Se observan valores de $V_{\mathrm{s}}>3 \mathrm{~km} / \mathrm{s}$ en las capas más superficiales de los modelos obtenidos en ambas estaciones mediante la inversión de FRs. La estructura media obtenida entre TRVA y USHA a partir de la inversión de la curva de dispersión para dicho trayecto, muestra valores de velocidad de onda $S$ en el rango 3.3-3.7 km/s, en los 2 primeros km de corteza. Dicho aumento de $\mathrm{V}_{\mathrm{s}}$ observado en TRVA y USHA en los primeros $\mathrm{km}$ de corteza, se corresponde con rocas del basamento Paleozoico presente en la zona entre ambas estaciones (Tassone et al., 2005). Además los valores altos de $V_{S}$ obtenidos en el sur de la Isla reflejarían la ausencia de sedimentos en dicha zona (Lawrence y Wiens, 2004). La estructura media de velocidad de onda S obtenida a partir de la inversión de velocidades de grupo, complementa a los modelos obtenidos a partir de la inversión de FRs cuyos valores representan la estructura local bajo cada estación. Considerando los valores de velocidad de onda $\mathrm{S}$ anteriormente mencionados se observa una tendencia de aumento de $\mathrm{V}_{\mathrm{S}}$ de norte a sur en las capas más superficiales de la corteza de TdF.

Los valores de velocidad de onda S son acordes con la geología presente en la zona de estudio, en particular con la litología superficial-subsuperficial. Las unidades más jóvenes, compuestas por sedimentos y rocas menos consolidadas se corresponden con velocidades de onda S menores. Hacia el sur, y considerando profundidades mayores, las unidades más antiguas y compuestas por 
litologías más consolidadas presentan mayores velocidades (Menichetti et al., 2008).

De todos los modelos obtenidos a partir de la inversión de FRs, el obtenido para el grupo ubicado al SO de la estación USHA, es el único que revela una zona de baja velocidad entre los 5 y $10 \mathrm{~km}$ de profundidad. La estructura local obtenida en la estación USHA es más compleja, y el análisis de FRs sugiere la presencia de una capa a los $5 \mathrm{~km}$ de profundidad inclinada $30^{\circ}$ al $\mathrm{NE}$ aproximadamente (más detalle en la sección 2.5.4.1 del Capítulo II). De manera análoga, la estructura media de velocidad de onda S calculada a partir de la inversión de la curva de dispersión obtenida para el trayecto TRVA-USHA, demuestra una disminución de velocidad en las capas más someras.

A profundidades un poco mayores (entre 5 y $8 \mathrm{~km}$ ), se observa un incremento de velocidad en varios de los modelos obtenidos con ambas técnicas. Dicha discontinuidad parece estar relacionada con el basamento de la zona de estudio, sugiriendo un aumento de su profundidad de norte a sur (Adaros Cárcamo, 2003; Tassone et al., 2005). En algunos de los modelos obtenidos mediante la técnica NA, se distingue además un elevado contraste positivo de velocidad entre los 15 y 17 km de profundidad. Dicha discontinuidad parece estar asociada con la presencia de un sub-basamento observado por Ludwig et al. (1965) en los perfiles de refracción sísmica realizados en el Estrecho de Magallanes cuyas profundidades alcanzaron la discontinuidad de Mohorovicic en algunos lugares (29.6 - $34.8 \mathrm{~km}$ ). Dicho incremento de velocidad no se observa en todas las estaciones, por lo que podría tratarse de una estructura local.

Los modelos obtenidos mediante todas las técnicas utilizadas, demuestran que la estructura cortical de la zona de estudio es compleja. Mediante las diferentes técnicas aplicadas fue posible la obtención de espesores corticales, relación de velocidades sísmicas y modelos de velocidad de onda S en los alrededores y bajo todas las estaciones utilizadas en este trabajo. La aplicación de todos los métodos mencionados fueron llevados a cabo considerando diferentes parámetros de inversión, factores de pesos, modelos iniciales de velocidad y diferentes estrategias de aplicación obteniendo una convergencia similar de los resultados en todos los casos. Esto demuestra la robustez de los modelos obtenidos generando confianza en los resultados presentados.

La zona de estudio está calificada como de moderada a elevada peligrosidad sísmica según el Instituto Nacional de Prevención Sísmica. En una zona sísmicamente activa, es importante contar con modelos de velocidad que se ajusten lo más posible a la estructura real de la zona para que la localización de los eventos sea con el menor error posible. Desde ese punto de vista, el presente trabajo aporta nueva información acerca de la estructura de la corteza en TdF. Se espera que estos modelos contribuyan en un futuro para el desarrollo de trabajos en la zona mediante otras técnicas, además de mejorar la localización de los sismos registrados en las estaciones de TdF implementando los modelos presentados en la relocalización de los mismos.

Por otro lado, es importante mencionar que una de las dificultades encontradas a lo largo de esta Tesis fue la falta de datos en ciertos períodos y la presencia de ruido en los registros. Con el fin de 
optimizar los datos y completar el análisis realizado con el método de FRs, se utilizó la técnica de interferometría de ruido sísmico. De esta manera se pudo restringir la estructura media entre los pares de estaciones mediante la dispersión de ondas superficiales. Como trabajo a futuro se considera la posibilidad de realizar un estudio de tomografía de ruido sísmico ambiental, teniendo en cuenta que los resultados obtenidos mediante la interferometría en este trabajo puede ayudar por ejemplo, para diseñar un despliegue de estaciones en TdF. 


\begin{tabular}{|c|c|c|c|c|c|c|}
\hline Fecha & Hora origen & Lat $\left({ }^{\circ}\right)$ & Long $\left({ }^{\circ}\right)$ & H (km) & $\mathbf{m}_{\mathbf{b}}$ & Estación Sismológica \\
\hline $2005 / 10 / 29$ & 04:05:56.04 & -45.21 & +96.90 & 8.0 & 6.5 & TRVA \\
\hline $2005 / 11 / 09$ & 11:33:13.19 & -1.020 & -76.94 & 248.0 & 5.9 & TRVA \\
\hline $2005 / 11 / 17$ & 19:26:54.49 & -22.36 & -67.89 & 147.0 & 6.8 & TRVA \\
\hline $2005 / 12 / 07$ & 23:32:51.55 & -30.01 & -177.64 & 21.0 & 6.4 & TRVA \\
\hline $2005 / 12 / 22$ & 04:28:23.59 & -54.50 & -135.66 & 10.0 & 5.5 & TRVA \\
\hline $2005 / 12 / 22$ & $12: 20: 02.94$ & -54.72 & -135.87 & 10.0 & 6.3 & TRVA \\
\hline $2005 / 12 / 23$ & $21: 47: 28.00$ & -1.390 & -77.52 & 192.0 & 6.1 & TRVA \\
\hline $2006 / 01 / 02$ & 22:13:40.49 & -19.93 & -178.18 & 582.0 & 7 & TRVA USHA \\
\hline $2006 / 02 / 04$ & 09:17:04.09 & 1.090 & -90.460 & 10.0 & 5.9 & TRVA \\
\hline $2006 / 02 / 22$ & 22:19:07.80 & -21.32 & +33.58 & 11.0 & 7 & TRVA \\
\hline $2006 / 02 / 23$ & 04:04:05.02 & -54.67 & 1.760 & 10.0 & 6.2 & TRVA \\
\hline $2006 / 03 / 31$ & 13:21:00.25 & -29.43 & -176.77 & 13.0 & 6.5 & TRVA \\
\hline $2006 / 05 / 03$ & 15:26:40.29 & -20.19 & -174.12 & 55.0 & 8 & TRVA \\
\hline $2006 / 05 / 16$ & 10:39:23.34 & -31.81 & -179.31 & 152.0 & 7.4 & TRVA \\
\hline $2006 / 06 / 27$ & $11: 24: 36.50$ & -21.29 & -68.52 & 122.0 & 5.5 & TRVA \\
\hline $2006 / 06 / 27$ & 13:03:09.22 & +14.99 & -94.14 & 9.0 & 5.8 & TRVA \\
\hline $2006 / 07 / 18$ & 16:02:16.79 & -20.08 & -178.43 & 587.0 & 5.7 & TRVA \\
\hline 2006/08/11 & 14:30:40.15 & +18.54 & -101.05 & 56.0 & 6.1 & TRVA \\
\hline $2006 / 08 / 25$ & 00:44:46.16 & -24.40 & -67.03 & 184.0 & 6.6 & TRVA \\
\hline $2006 / 09 / 24$ & $22: 56: 21.07$ & -17.74 & +41.81 & 6.0 & 5.7 & TRVA \\
\hline $2006 / 09 / 28$ & 06:22:09.73 & -16.59 & -172.03 & 28.0 & 6.9 & TRVA \\
\hline $2006 / 09 / 29$ & 18:23:05.94 & +10.81 & -61.76 & 52.0 & 5.5 & TRVA \\
\hline $2006 / 09 / 30$ & $16: 26: 56.12$ & -15.59 & -73.16 & 107.0 & 6 & TRVA \\
\hline $2006 / 12 / 15$ & 23:57:10.42 & -35.33 & -105.75 & 10.0 & 5.8 & TRVA \\
\hline $2007 / 01 / 08$ & $20: 52: 20.64$ & -18.58 & -177.85 & 406.0 & 6.3 & TRVA DSPA \\
\hline $2007 / 01 / 30$ & 04:54:50.57 & -54.74 & +146.3 & 11.0 & 6.9 & TRVA DSPA USHA \\
\hline $2007 / 01 / 31$ & 03:15:52.29 & -29.78 & -178.0 & 34.0 & 6.5 & TRVA DPSA USHA \\
\hline $2007 / 02 / 04$ & 21:17:46.79 & -55.88 & -122.72 & 10.0 & 6.2 & DSPA \\
\hline $2007 / 02 / 14$ & 01:29:07.19 & -29.59 & -112.05 & 10.0 & 5.9 & TRVA DSPA USHA \\
\hline $2007 / 02 / 19$ & 19:24:10.63 & -23.05 & -70.47 & 10.0 & 5.5 & DSPA \\
\hline $2007 / 02 / 24$ & 02:36:23.65 & -07.01 & -80.49 & 23.0 & 6.3 & TRVA DSPA \\
\hline $2007 / 03 / 01$ & 23:11:52.07 & +26.58 & -44.59 & 10.0 & 6.0 & TRVA DSPA \\
\hline $2007 / 03 / 08$ & 11:14:31.51 & -58.22 & -7.610 & 10.0 & 6.2 & TRVA DSPA USHA \\
\hline $2007 / 03 / 10$ & 14:07:08.60 & -23.29 & -69.71 & 50.0 & 5.5 & DSPA \\
\hline 2007/03/13 & 02:59:04.40 & +26.26 & -110.54 & 26.0 & 6.0 & DSPA \\
\hline 2007/03/18 & 02:11:05.30 & +04.59 & -78.49 & 7.0 & 6.2 & TRVA DSPA \\
\hline $2007 / 03 / 24$ & 19:13:50.58 & -19.72 & -70.14 & 30.0 & 5.7 & TRVA DSPA \\
\hline $2007 / 03 / 31$ & 12:49:03.71 & -56.08 & -123.27 & 10.0 & 6.2 & USHA \\
\hline $2007 / 04 / 06$ & 05:54:20.42 & -55.37 & -123.64 & 10.0 & 5.9 & USHA \\
\hline $2007 / 04 / 13$ & 18:24:19.16 & -35.05 & -108.84 & 10.0 & 6.1 & TRVA DSPA \\
\hline
\end{tabular}




\begin{tabular}{|c|c|c|c|c|c|c|}
\hline 2007/05/04 & 12:06:51.75 & -01.41 & -14.920 & 7.0 & 6.2 & USHA \\
\hline 2007/05/25 & $17: 47: 31.26$ & -24.22 & -67.03 & 180.0 & 5.9 & TRVA \\
\hline 2007/06/13 & $19: 29: 40.18$ & +13.55 & -90.62 & 23.0 & 6.7 & DSPA \\
\hline 2007/06/24 & $00: 25: 18.40$ & -55.65 & -02.63 & 10.0 & 6.5 & DSPA \\
\hline 2007/07/03 & 08:26:00.81 & +00.71 & -30.27 & 10.0 & 6.3 & DSPA \\
\hline 2007/07/12 & $05: 23: 49.34$ & -07.63 & -74.38 & 152.0 & 6.1 & DSPA USHA \\
\hline 2007/07/13 & 16:33:35.72 & -55.60 & -128.52 & 10.0 & 5.7 & DSPA \\
\hline 2007/07/17 & 09:39:27.81 & -26.21 & -177.74 & 10.0 & 6.1 & USHA \\
\hline 2007/07/18 & 00:07:35.46 & -26.30 & -177.74 & 10.0 & 6.1 & DSPA \\
\hline 2007/07/21 & $15: 34: 52.60$ & -22.15 & -65.78 & 289.0 & 6.4 & DSPA USHA \\
\hline 2007/07/21 & 20:40:53.79 & -55.09 & -129.42 & 10.0 & 5.7 & DSPA \\
\hline 2007/07/31 & 22:55:31.13 & -00.16 & -17.80 & 11.0 & 6.2 & DSPA \\
\hline 2007/08/15 & 23:40:57.89 & -13.39 & -76.60 & 39.0 & 8.0 & DSPA USHA \\
\hline 2007/08/16 & 05:16:56.15 & -14.28 & -76.13 & 23.0 & 6.4 & DSPA \\
\hline 2007/08/17 & 13:18:13.22 & -13.60 & -76.60 & 48.0 & 5.9 & DSPA USHA \\
\hline 2007/08/18 & 02:52:35.40 & -13.81 & -76.290 & 30.0 & 6.0 & USHA \\
\hline 2007/08/19 & 01:22:38.90 & -13.62 & -76.62 & 11.0 & 5.8 & DSPA USHAA \\
\hline 2007/08/20 & $22: 42: 28.53$ & +08.04 & -39.250 & 6.0 & 6.5 & USHA \\
\hline 2007/09/01 & 19:14:22.64 & +24.90 & -109.69 & 9.0 & 6.1 & DSPA \\
\hline 2007/09/10 & 01:49:11.78 & +02.97 & -77.96 & 15.0 & 6.8 & TRVA DSPA USHA \\
\hline 2007/09/30 & 05:23:34.07 & -49.27 & +164.12 & 10.0 & 7.4 & TRVA DSPA USHA \\
\hline 2007/09/30 & 09:47:51.97 & -49.14 & +164.11 & 18.0 & 6.6 & DSPA \\
\hline 2007/10/15 & $12: 29: 34.86$ & -44.80 & +167.55 & 18.0 & 6.8 & TRVA \\
\hline 2007/11/02 & $22: 31: 43.88$ & -55.47 & -128.97 & 10.0 & 6.1 & TRVA USHA \\
\hline 2007/11/10 & 01:13:29.35 & -51.78 & +161.32 & 10.0 & 6.6 & TRVA USHA \\
\hline 2007/11/14 & $15: 40: 50.53$ & -22.25 & -69.89 & 40.0 & 7.7 & TRVA USA \\
\hline 2007/11/15 & 15:05:58.35 & -22.92 & -70240 & 26.0 & 6.8 & USHA \\
\hline 2007/11/16 & 03:13:00.12 & -2.310 & -77.84 & 122.0 & 6.8 & TRVA USHA \\
\hline 2007/11/17 & 03:07:32.50 & -23.05 & -70.29 & 34.0 & 5.5 & TRVA \\
\hline 2007/11/17 & $17: 54: 32.77$ & -23.06 & -70.60 & 14.0 & 5.9 & TRVA USHA \\
\hline 2007/11/18 & 05:40:12.04 & -22.64 & -66.32 & 246.0 & 6.0 & TRVA \\
\hline 2007/11/18 & $12: 15: 40.73$ & -23.10 & -70.55 & 33.0 & 5.7 & TRVA \\
\hline 2007/11/19 & 23:30:28.90 & -22.79 & -70.77 & 29.0 & 5.9 & TRVA \\
\hline 2007/11/20 & 17:55:51.53 & -22.92 & -70.48 & 15.0 & 6.1 & TRVA USHA \\
\hline 2007/11/21 & 12:55:05.06 & -24.81 & -68.47 & 115.0 & 5.8 & TRVA \\
\hline 2007/11/29 & $19: 00: 20.42$ & +14.94 & -61.27 & 156.0 & 7.4 & TRVA USHA \\
\hline 2007/12/06 & $17: 12: 03.22$ & +22.69 & -45.11 & 10.0 & 5.8 & TRVA \\
\hline 2007/12/07 & 06:19:35.16 & -56.21 & -139.29 & 10.0 & 5.5 & TRVA \\
\hline 2007/12/09 & $07: 28: 20.82$ & -26.00 & -177.51 & 152.0 & 6.6 & TRVA DSPA \\
\hline 2007/12/13 & 05:20:21.85 & -23.16 & -70.48 & 15.0 & 6.0 & TRVA DSPA USHA \\
\hline 2007/12/13 & 07:23:39.89 & -23.20 & -70.55 & 16.0 & 6.2 & DSPA USHA \\
\hline 2007/12/16 & 08:09:17.93 & -22.95 & -70.18 & 45.0 & 6.7 & TRVA USHA \\
\hline 2007/12/20 & 07:55:15.84 & -39.01 & +178.29 & 20.0 & 6.6 & TRVA USHA \\
\hline 2007/12/26 & 23:40:53.1 & -22.45 & -68.63 & 106.0 & 5.5 & TRVA USHA \\
\hline 2008/01/05 & 07:29:33.50 & -22.82 & -68.48 & 103.0 & 5.7 & TRVA \\
\hline 2008/01/15 & 17:52:15.69 & -21.98 & -179.54 & 5.97 & 6.5 & TRVA \\
\hline 2008/01/20 & 06:08:56.72 & -35.02 & -112.02 & 10.0 & 5.6 & TRVA ELCA \\
\hline 2008/01/21 & $12: 24: 27.06$ & -34.86 & -111.85 & 10.0 & 5.8 & ELCA DSPA USHA \\
\hline 2008/01/22 & $10: 49: 21.80$ & -15.42 & -175.59 & 10.0 & 6.1 & ELCA \\
\hline
\end{tabular}




\begin{tabular}{|c|c|c|c|c|c|c|}
\hline $08 / 02 / 04$ & $17: 01: 29.98$ & -20.17 & -70.04 & 35.0 & 6.3 & TRVA ELCA DSPA \\
\hline 2008/02/08 & 09:38:14.10 & +10.67 & -41.90 & 9.0 & 6.9 & TRVA DSPA \\
\hline $2008 / 03 / 24$ & 20:39:07.63 & -20.04 & -68.96 & 120.0 & 6.2 & TRVA \\
\hline $2008 / 04 / 12$ & $00: 30: 12.6$ & -55.66 & +158.45 & 16.0 & 7.1 & TRVA ELCA USHA \\
\hline $2008 / 04 / 15$ & 03:03:04.66 & +13.56 & -90.60 & 33.0 & 6.1 & TRVA \\
\hline $2008 / 04 / 24$ & $12: 14: 49.92$ & -01.18 & -23.470 & 10 & 6.5 & USHA \\
\hline $2008 / 04 / 26$ & 23:34:49.39 & -49.09 & +164.12 & 10.0 & 6.1 & TRVA \\
\hline $2008 / 05 / 14$ & $21: 47: 40.28$ & -56.77 & -142.31 & 10.0 & 5.7 & TRVA \\
\hline $2008 / 05 / 19$ & 03:16:13.62 & -47.78 & +31.97 & 10.0 & 5.9 & TRVA DSPA USHA \\
\hline $2008 / 05 / 23$ & $19: 35: 34.78$ & +07.31 & -34.900 & 8 & 6.5 & USHA \\
\hline 2008/06/01 & 14:31:03.01 & -59.38 & +149.66 & 10.0 & 6.5 & DSPA USHA \\
\hline 2008/06/15 & 08:37:17.20 & -36.62 & -107.45 & 10.0 & 6.0 & DSPA \\
\hline 2008/07/08 & 09:13:07.46 & -15.99 & -71.75 & 123.0 & 6.2 & TRVA DSPA \\
\hline 2008/07/15 & 05:07:11.90 & -47.37 & -12.17 & 10.0 & 5.7 & TRVA \\
\hline 2008/07/19 & $22: 39: 52.7$ & -17.34 & -177.31 & 391.0 & 6.4 & TRVA DSPA \\
\hline 2008/08/09 & 06:01:48.51 & -60.65 & +153.77 & 10.0 & 6.5 & ELCA \\
\hline 2008/08/26 & 21:00:36.61 & -7.640 & -74.38 & 154.0 & 6.4 & TRVA ELCA DSPA \\
\hline $2008 / 08 / 28$ & 15:22:23.2 & $-0,25$ & -17.36 & 12.0 & 6.3 & TRVA ELCA DSPA \\
\hline 2008/09/01 & 04:00:39.43 & -25.39 & -177.64 & 171.0 & 6.0 & TRVA \\
\hline $2008 / 09 / 24$ & 02:33:05.56 & +17.61 & -105.50 & 12.0 & 6.4 & USHA \\
\hline $2008 / 09 / 29$ & $15: 19: 31.59$ & -29.76 & -177.68 & 36.0 & 7 & TRVA DPSA USHA \\
\hline 2008/10/11 & $10: 40: 14.06$ & +19.16 & -64.83 & 23.0 & 6.1 & TRVA \\
\hline 2008/10/12 & $20: 55: 41.46$ & -20.12 & -64.97 & 352.0 & 6.2 & DSPA \\
\hline 2008/10/16 & $19: 41: 25.72$ & +14.42 & -92.36 & 24.0 & 6.7 & TRVA DSPA USHA \\
\hline 2008/10/19 & 05:10:33.91 & -21.86 & -173.82 & 29.0 & 6.9 & VA ELCA USHA DS \\
\hline $2008 / 10 / 22$ & $12: 55: 57.39$ & -18.41 & -175.35 & 233.0 & 6.4 & TRVA DSPA \\
\hline $2008 / 11 / 14$ & 02:05:09.90 & -53.79 & +08.73 & 12.0 & 5.9 & TRVA \\
\hline 2008/11/15 & 6.62 & +13.05 & -88.75 & 48.0 & 5 & ELCA D \\
\hline 2008/11/19 & 0.79 & +08.27 & -82.97 & 32.0 & 6.3 & DSPA \\
\hline $2008 / 11 / 22$ & $16: 0$ & -22.52 & +171.2 & 57.0 & 6.4 & TRVA ELCA DSPA \\
\hline 2008 & $18: 3$ & -53.01 & +106.82 & 11.0 & 6.3 & TRVA DSPA \\
\hline 2008 & $06: 2$ & -31.23 & -176.92 & 18.0 & 6.8 & TRVA ELCA USHA DSPA \\
\hline $2008 / 12 / 13$ & $08: 4$ & -48.98 & +123.40 & 10.0 & 5.9 & TRVA DSPA USHA \\
\hline 2008/12/18 & $20: 5^{\prime}$ & -23.50 & -180.0 & 542.0 & 5.6 & TRVA DSPA USHA \\
\hline $2008 / 12 / 20$ & 21:05:16.20 & -31.19 & -13.34 & 4.0 & 5.8 & TRVA DSPA \\
\hline 2009/01/01 & $06: 27: 51.22$ & -34.84 & -107.65 & 10.0 & 5.8 & TRVA ELCA DSPA \\
\hline 2009/01/08 & 19:21:35.61 & +10.16 & -84.20 & 14.0 & 6.1 & TRVA DSPA USHA \\
\hline 2009/01/15 & $07: 27: 20.29$ & -22.35 & +170.63 & 27.0 & 6.7 & TRVA DSPA \\
\hline 2009/01/18 & $14: 11: 48.86$ & -30.20 & -177.95 & 33.0 & 6.4 & TRVA ELCAUSHA DSPA \\
\hline 2009/01/19 & 03:35:18.84 & -22.60 & +170.91 & 12.0 & 6.6 & DSPA \\
\hline 2009/02/02 & $17: 53: 21.80$ & -13.58 & -76.56 & 21.0 & 6.0 & TRVA DSPA \\
\hline $2009 / 02 / 17$ & 03:30:53.33 & -30.72 & -178.62 & 13.0 & 6.0 & TRVA DSPA \\
\hline $2009 / 02 / 18$ & 03:07:50.34 & -52.97 & +20.91 & 10.0 & 5.9 & TRVA DSPA \\
\hline $2009 / 02 / 18$ & 21:53:45.16 & -27.42 & -176.33 & 25.0 & 7 & TRVA \\
\hline 2009/01/19 & 03:35:18.84 & -22.60 & +170.91 & 12.0 & 6.6 & TRVA \\
\hline 2009/03/11 & 21:03:58.98 & +08.49 & -83.21 & 17.0 & 5.9 & TRVA DSPA \\
\hline 2009/03/12 & $23: 23: 34.82$ & +05.69 & -82.77 & 9.0 & 6.3 & TRVA \\
\hline $2009 / 03 / 15$ & 03:14:31.91 & 2.430 & -95.12 & 10.0 & 5.8 & TRVA \\
\hline $2009 / 03 / 16$ & 07:13:36.09 & -55.14 & -129.7 & 10.0 & 5.9 & TRVA DSPA \\
\hline
\end{tabular}




\begin{tabular}{|c|c|c|c|c|c|c|}
\hline 2009/03/19 & $18: 17: 40.47$ & -23.04 & -174.66 & 31.0 & 7.6 & TRVA DSPA USHA \\
\hline 2009/04/17 & 02:08:08.71 & -19.58 & -70480 & 25.0 & 6.1 & ELCA DSPA USHA \\
\hline 2009/05/10 & $01: 16: 06.96$ & 1.390 & -85170 & 6.0 & 6.1 & ELCA DSPA USHA \\
\hline 2009/05/16 & $00: 53: 51.25$ & -31.55 & -178.83 & 43.0 & 6.5 & ELCA DSPA \\
\hline 2009/05/24 & 00:58:02.11 & -31.48 & -177.68 & 4.0 & 6.0 & DSPA USHA \\
\hline 2009/05/28 & 08:24:46.56 & +16.73 & -86.220 & 19.0 & 7.3 & ELCA DSPA USHA \\
\hline 2009/06/16 & 20:05:56.99 & -54.37 & +05.87 & 10.0 & 6.1 & DSPA \\
\hline 2009/07/08 & $19: 23: 37.73$ & -36.00 & -102.69 & 12.0 & 6.0 & ELCA DSPA \\
\hline 2009/07/15 & $09: 22: 29.03$ & -45.76 & +166.56 & 12.0 & 7.8 & ELCA DSPA \\
\hline $2009 / 07 / 30$ & $20: 05: 35.65$ & -20.85 & -174.26 & 14.0 & 5.8 & DSPA \\
\hline 2009/08/01 & 13:33:29.95 & -56.23 & -124.30 & 10.0 & 6.1 & ELCA \\
\hline 2009/08/05 & 08:31:40.48 & -45.55 & +166.36 & 10.0 & 6.1 & DSPA \\
\hline 2009/08/30 & 14:51:32.89 & -15.22 & -172.57 & 11.0 & 6.6 & ELCA DSPA \\
\hline 2009/09/12 & $20: 06: 25.47$ & +10.71 & -67.930 & 14.0 & 6.4 & ELCA \\
\hline 2009/09/17 & 23:21:38.52 & -29.14 & -112.27 & 10.0 & 6.2 & DSPA \\
\hline 2009/09/24 & 07:16:20.26 & +18.83 & -107.34 & 13.0 & 6.4 & DSPA \\
\hline 2009/09/29 & 17:48:10.99 & -15.49 & -172.10 & 18.0 & 8.1 & ELCA DSPA \\
\hline 2009/11/13 & 03:05:57.22 & -19.39 & -70.320 & 27.0 & 6.5 & ELCA DSPA \\
\hline 2009/11/13 & $07: 27: 13.36$ & -17.92 & -64.10 & 608.0 & 5.8 & DSPA \\
\hline 2009/11/14 & $19: 44: 29.93$ & -22.97 & -66.64 & 220.0 & 6.2 & DSPA \\
\hline $2009 / 11 / 24$ & $12: 47: 15.66$ & -20.71 & -174.04 & 18.0 & 6.8 & ELCA \\
\hline 2009/12/03 & $06: 12: 32.49$ & -56.48 & -122.32 & 10.0 & 6.0 & ELCA DSPA \\
\hline 2009/12/09 & 09:46:03.30 & -22.15 & +170.96 & 45.0 & 6.4 & ELCADSPA \\
\hline 2009/12/31 & 09:23:24.01 & -59.40 & -150.98 & 10.0 & 6.0 & ELCA DSPA \\
\hline 2010/01/12 & 21:53:10.06 & +18.44 & -72.57 & 13.0 & 7 & TRVA ELCA \\
\hline 2010/01/20 & 11:03:43.49 & +18.42 & -72.82 & 10.0 & 5.9 & TRVA DSPA \\
\hline $2010 / 01 / 28$ & 08:04:14.25 & -23.36 & -66.71 & 208.0 & 5.9 & DSPA \\
\hline 2010/02/05 & 06:59:05.05 & -47.91 & +99.59 & 1.0 & 6.2 & TRVA ELCA DSPA \\
\hline 2010/02/13 & 02:34:28.86 & -21.90 & -174.77 & 11.0 & 6.1 & TRVA DSPA \\
\hline 2010/02/19 & 11:13;19.81 & -21.84 & -175.12 & 10.0 & 5.8 & DSPA \\
\hline $2010 / 02 / 22$ & 07:00:52.91 & -23.64 & -176.04 & 25.0 & 6.0 & DSPA \\
\hline $2010 / 02 / 27$ & 06:34:16.29 & -54.03 & -133.64 & 10.0 & 5.6 & ELCA DSPA \\
\hline 2010/03/04 & $22: 39: 26.53$ & -22.23 & -68.33 & 114.0 & 6.4 & TRVA DSPA \\
\hline 2010/03/07 & 07:05:23.96 & -16.24 & -115.3 & 14.0 & 6.3 & TRVA ELCA DSPA \\
\hline 2010/03/18 & 09:14:05.86 & -23.32 & -177.18 & 158.0 & 5.6 & TRVA ELCA DSPA \\
\hline 2010/05/19 & $10: 30: 10.40$ & -54.80 & -135.25 & 10.0 & 6.0 & TRVA \\
\hline 2010/05/24 & 16:18:29.09 & -08.09 & -71.56 & 581.0 & 6.5 & TRVA \\
\hline 2010/07/12 & $00: 11: 21.25$ & -22.15 & -68.22 & 115.0 & 6.3 & TRVA \\
\hline 2010/09/03 & $16: 35: 47.77$ & -43.52 & +171.83 & 12.0 & 7 & TRVA ELCA DSPA \\
\hline 2010/11/10 & 04:05:24.41 & -45.46 & +96.39 & 10.0 & 6.5 & TRVA ELCA \\
\hline 2010/11/12 & 19:01:29.97 & -35.96 & -102.21 & 10.0 & 5.8 & TRVA \\
\hline 2010/12/07 & $04: 27: 22.04$ & -57.93 & -07.47 & 10.0 & 5.9 & TRVA ELCA \\
\hline $2010 / 12 / 28$ & 08:34:17.55 & -23.41 & -179.80 & 551.0 & 6.3 & TRVA DSPA \\
\hline
\end{tabular}

Tabla A.1: Telesismos seleccionados para el cálculo de las FRs en TdF (se indican en rojo y azul en la Figura 2.7 del Capítulo II). En la tabla se indica la fecha, hora origen, latitud, longitud, profundidad focal (H) y la magnitud (mb) de los eventos. Además se indica la estación que lo registró. En muchos casos los telesismos fueron registrados por más de una estación. 


\section{APÉNDICE B}

En las Figuras B.1 a B.4 se muestran las velocidades de grupo obtenidas a partir de la correlación cruzada de los registros de ruido sísmico obtenido en las estaciones DSPA, TRVA, USHA y ELCA. Se consideró el apilamiento lineal de la correlación cruzada (PCC) de un año de datos. El gráfico inferior en todos los casos está normalizado por la frecuencia.
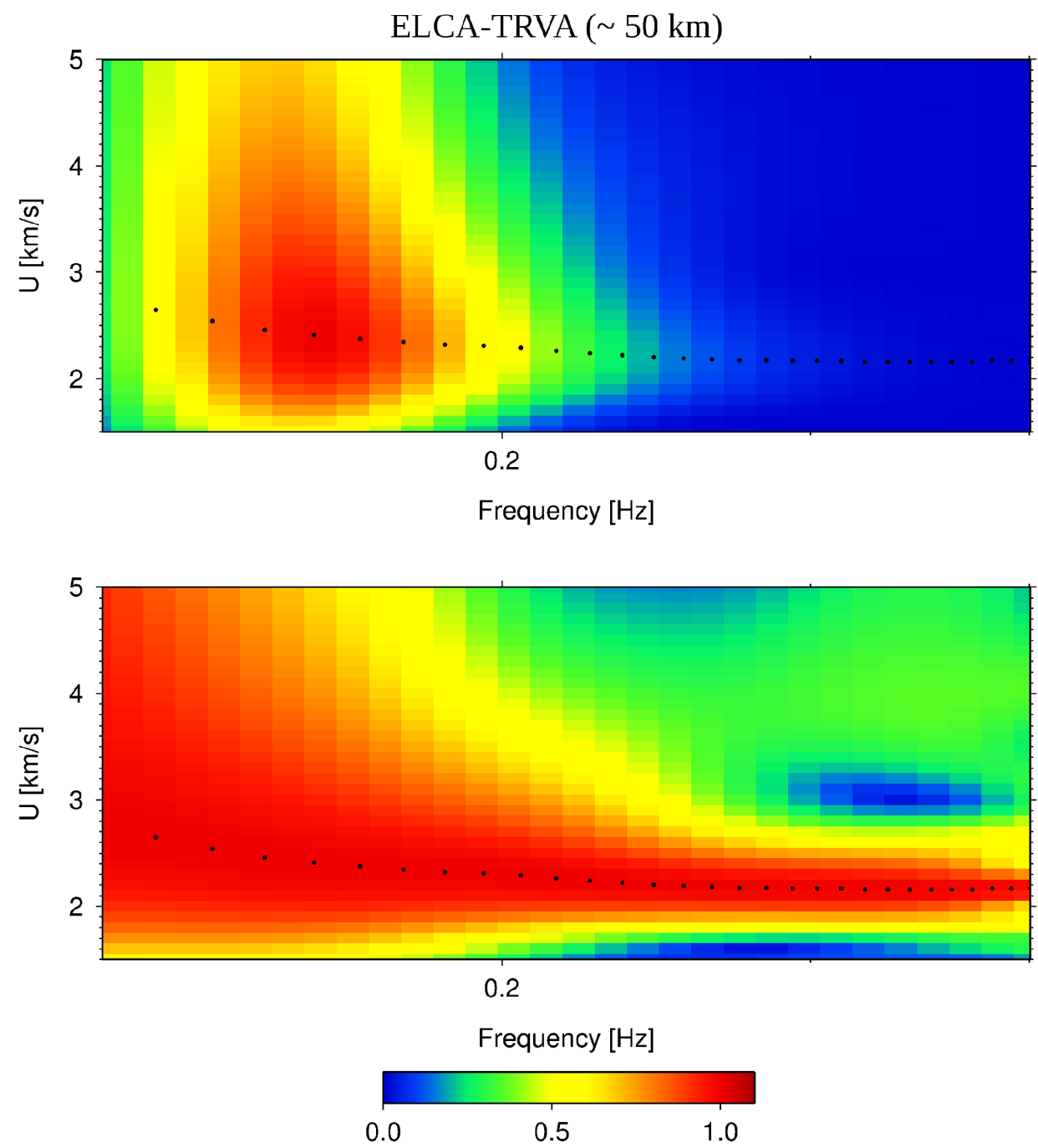

Figura B.1: Curva de dispersión obtenida a partir de la correlación cruzada entre las estaciones ELCA y TRVA. Los puntos negros indican los valores de velocidad de grupo obtenidos en función de la frecuencia. 

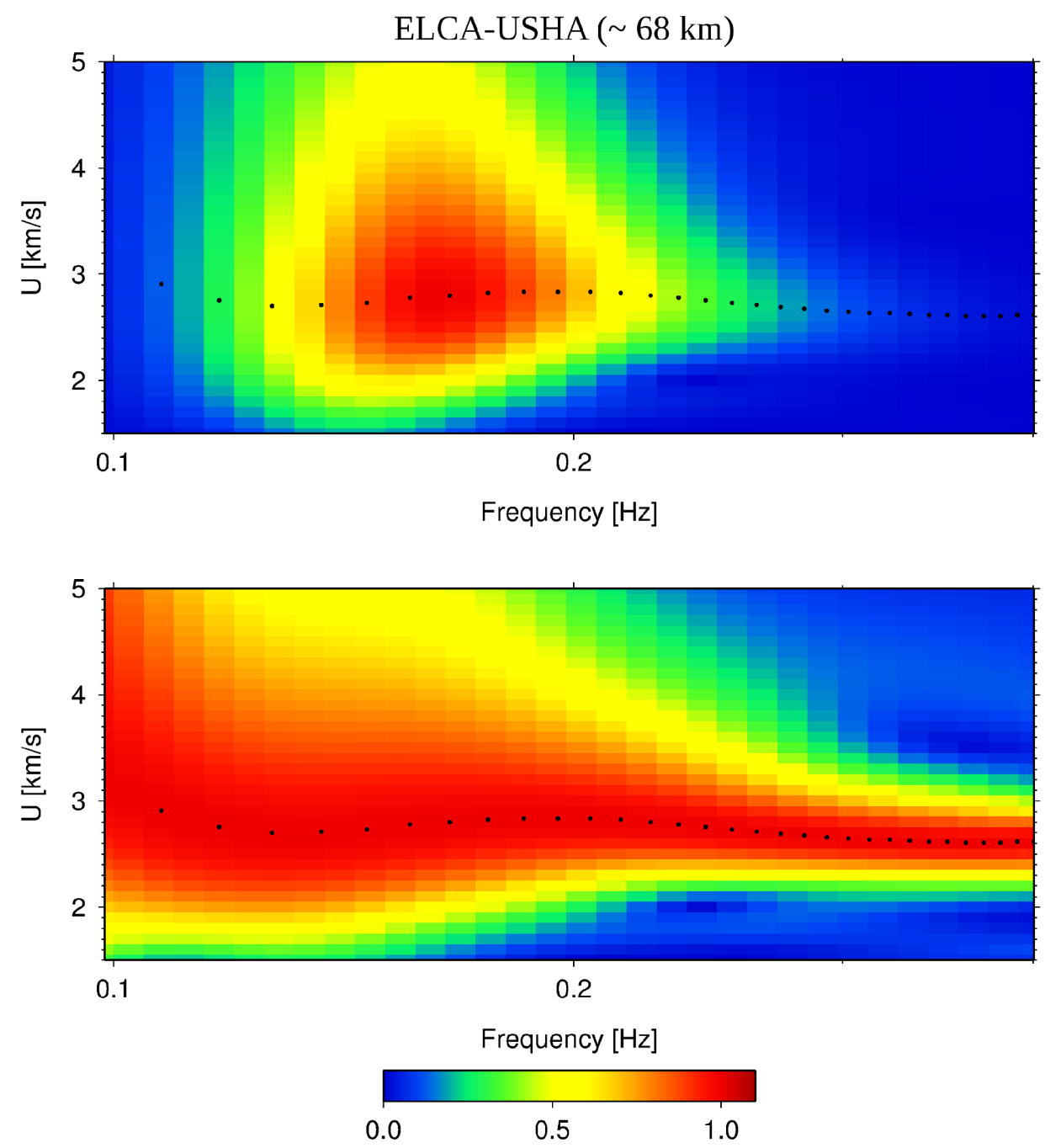

Figura B.2: Curva de dispersión obtenida a partir de la correlación cruzada entre las estaciones ELCA y USHA. Los puntos negros indican los valores de velocidad de grupo obtenidos en función de la frecuencia. 

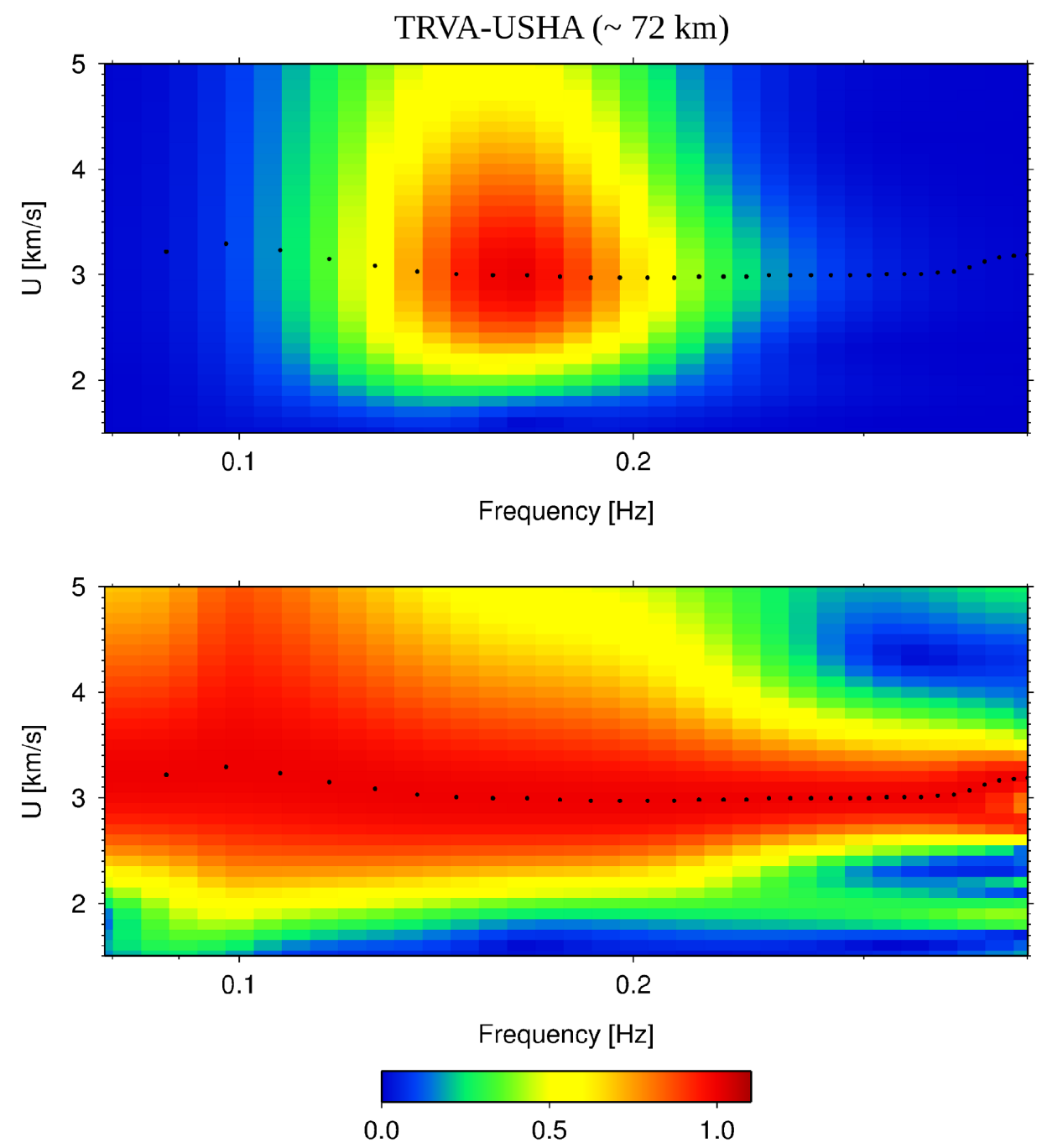

Figura B.3: Curva de dispersión obtenida a partir de la correlación cruzada entre las estaciones TRVA y USHA. Los puntos negros indican los valores de velocidad de grupo obtenidos en función de la frecuencia. 

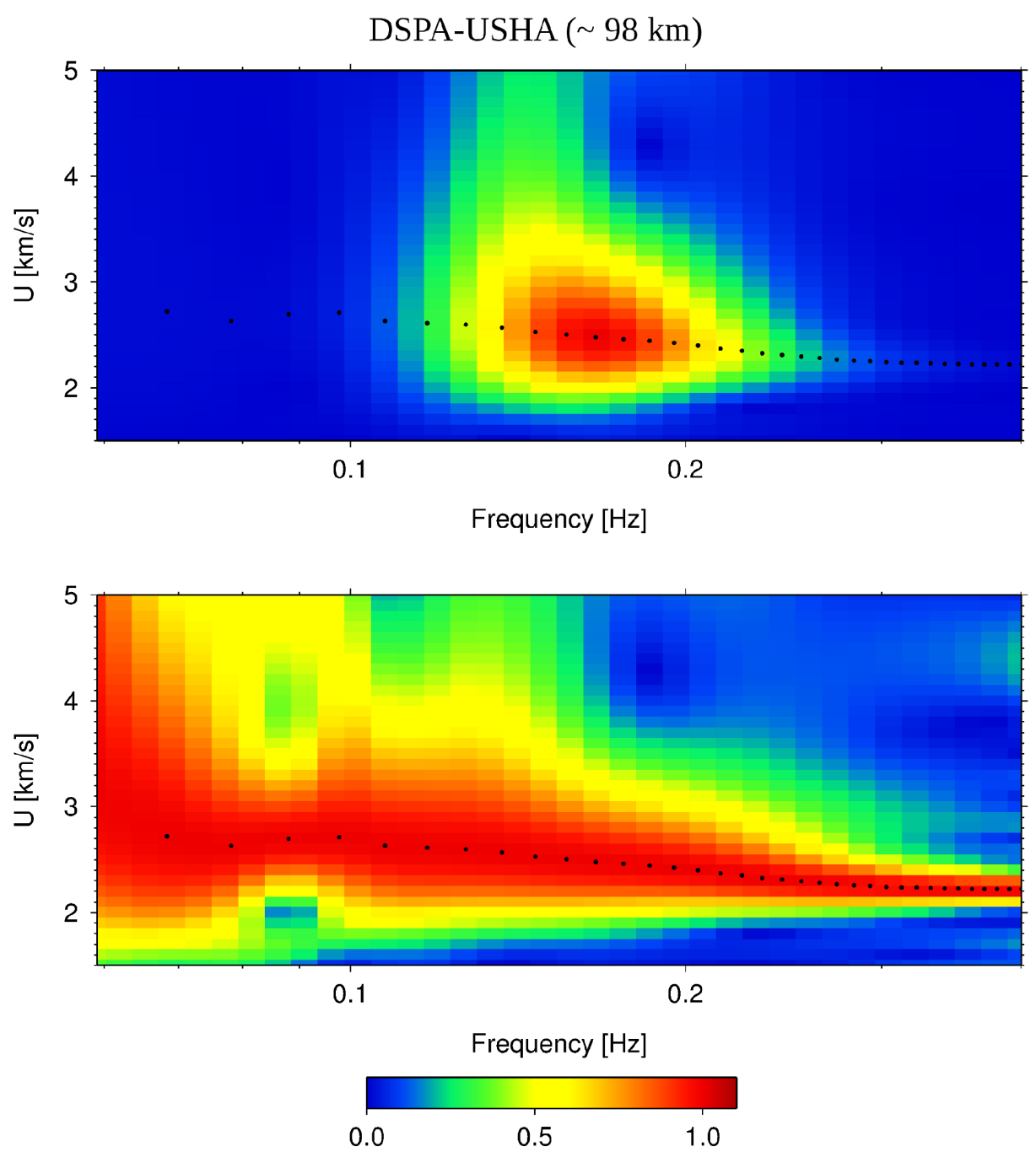

Figura B.4: Curva de dispersión obtenida a partir de la correlación cruzada entre las estaciones DSPA y USHA. Los puntos negros indican los valores de velocidad de grupo obtenidos en función de la frecuencia. 


\section{REFERENCIAS}

Abers, G.A., X. Hu, L.R. Sykes, 1995. Source scaling of earthquakes in the Shumagin region, Alaska: time-domain inversions of regional waveforms, Geophys. J. Int. 123, 41-58.

Abt, D.L., K.M. Fischer, S.W. French, H.A. Ford, H. Yuan and B. Romanowicz, 2010. North American lithospheric discontinuity structure imaged by Ps and Sp receiver functions. J. Geophys. Res. 115, B09301, doi:10.1029/2009JB006914.

Adaros Cárcamo, R.E., 2003. Tesis para optar el grado de Magister en Ciencias, Mención Geofísica. Sismicidad y Tectónica del extremo sur de Chile, Universidad de Chille, pp. 90.

Ai, Y., D. Zhaob, X. Gaoc, W. Xua, 2005. The crust and upper mantle discontinuity structure beneath Alaska inferred from receiver functions. Physics of the Earth and Planetary Interiors ,150, 339-350.

Akpan, O., A. Nyblade, C. Okereke, M. Oden, E. Emry, J. Julià, 2016. Crustal structure of Nigeria and Southern Ghana, West Africa from P-wave receiver functions. Tectonophysics, 676, 26, 250-260.

Ammirati, J.B., P. Alvarado, M. Perarnau, M. Saez, G. Monsalvo, 2013. Crustal structure of the Central Precordillera of San Juan, Argentina (31S) using teleseismic receiver functions. Journal of South American Earth Sciences, 46, 100-109.

Ammirati, J.B., P. Alvarado, S. Beck, 2015. A lithospheric velocity model for the flat slab region of Argentina from joint inversion of Rayleigh wave phase velocity dispersion and teleseismic receiver functions. Geophysical Journal International, 202(1), 224-241.

Ammon, C.J., G.E. Randall, G. Zandt, 1990. On the nonuniqueness of receiver function inversions. J. Geophys. Res., 95, 15303-15308.

Ammon, C.J., 1991. The isolation of receiver effects from teleseismic P waveforms. Bull. Seismol. Soc. Am. 81, 2504-2510.

Ammon, C.J., G. Zandt, 1993. Receiver structure beneath the southern Mojave block, California. Bull. Seism. Soc. Am., 83, 737-755.

Ammon, C.J., 2006. Isolating the Receiver Response Langston's Source Equalization Procedure, http://eqseis.geosc.psu.edu/ cammon/HTML/RftnDocs/seq01.html.

Angus, D. A., D.C. Wilson, E. Sandvol, J.F. Ni, 2006. Lithospheric structure of the Arabian and Eurasian collision zone in eastern Turkey from S-wave receiver functions. Geophysical Journal International, 166, 1335-1346. 
Ardhuin, F., E. Stutzmann, M. Schimmel, A. Mangeney, 2011. Ocean wave sources of seismic noise. J. Geophys. Res. , 116, C09004, doi:10.1029/2011JC006952.

Assumpçao, M., D. James, A. Snoke, 2002. Crustal thicknesses in SE Brazilian Shield by receiver function analysis: Implications for isostatic compensation. Journal of Geophysical Research: Solid Earth 107 (B1), Pages ESE 2-1-ESE 2-14.

Barker, P.F., J. Burrell, 1977. The opening of Drake Passage. Mar. Geol. 25, 15-34.

Bensen, G.D., M.H. Ritzwoller, M.P. Barmin, A.L. Levshin, F.C. Lin, M.P. Moschetti, N.M. Shapiro, Y. Yang, 2007. Processing seismic ambient noise data to obtain reliable broadband surface wave dispersion measurements. Geophys. J. Int. , 169 (3), 1239-1260.

Bonorino, G., V. Rinaldi, L. Abascal, P. Alvarado, G. Bujalesky, A. Guell, A., 2012. Paleoseismicity and seismic hazard in southern Patagonia (Argentina-Chile; $50^{\circ}-55^{\circ} \mathrm{S}$ ); the role of the Magallanes-Fagnano transform fault. Natural Hazards, vol. 61, pp. 337-349.

Bormann, P., 2002. New Manual of Seismological Observatory Practice, IASPEI, Vol. 1, Pp. 1252.

Bostock, M. G., 1996. Ps conversions from the upper mantle transition zone beneath the Canadian land mass. L. Geophys. Res., 101, 8393-8402.

Bromirski P.D., 2001. Vibrations from the "Perfect Storm”. Geochem. Geophys. Geosyst., 2, 1030, doi:10.1029/2000GC000119.

Bromirski P., F. Duennebier, 2002. The near-coastal microseism spectrum: Spatial and temporal wave climate relationships. Journal of Geophysical Research, 107 (B8), 2166.

Buffoni, C., 2008. Tesis de Grado “Estudio de sismicidad en la Isla de Tierra del Fuego”. Facultad de Ciencias Astronómicas y Geofísicas, UNLP, pp. 72.

Buffoni, C., N.C. Sabbione, G. Connon, J.L. Hormaechea, 2009. Localización de hipocentros y determinación de su magnitud en Tierra del Fuego y zonas aledañas. Geoacta 34, 75-85.

Burdick, L.J., C.A. Langston, 1977. Modeling crustal-structure through the use of converted phases in teleseismic body-waveforms. Bull. Seismol. Soc. Am. 67, 677-691.

Caminos, R., Nullo, F., 1979. Descripción geológica de la Hoja 67e. Isla de Los Estados. Boletín del Servicio Geológico Nacional (Argentina) 175, 1-175.

Caminos, R., Hall, M.A., Lapido, O., Lizuaín, A., Page, R., Ramos, V., 1981. Reconocimiento geológico de los Andes Fueguinos, territorio Nacional de Tierra del Fuego, IIX Congreso Geológico Argentino, Buenos Aires Actas. III, pp. 759-786.

Campillo, M., A. Paul, 2003. Long-range correlations in the diffuse seismic coda. Science, 299 (5606), 547-549.

Cassidy, J.F. 1992. Numerical experiments in broadband receiver function analysis. Bull. Seismol. Soc. Am. 82, 1453-1474.

Cassidy, J.F., 1995. A comparison of the receiver structure beneath stations of the Canadian National Seismograph Network. Canadian Journal of Earth Sciences, 1995, 32, 938-951.

Cassidy, J.F., R.M. Ellis, C. Karavas, G.C. Rogers, 1998. The northern limit of the subducted Juan 
de Fuca plate system. Journal of Geophysical Research 103, doi: 10.1029/98JB02140.

Chang, S.J., C.E. Baag, C. A. Langston, 2004. Joint analysis of teleseismic receiver functions and surface wave dispersion using the genetic algorithm. Bulletin of the Seismological Society of America, 94(2), 691-704.

Clayton, R.W., R.A. Wiggins, 1976. Source shape estimation and deconvolution of teleseismic body waves, Geophys. J. R. astr. Soc, 47, 151-177.

Clitheroe, G., O. Gudmundsson, B.L.N. Kennett, 2000. The crustal thickness of Australia. Journal of Geophysical Research, 105(13), 697-13.

Coronato, A., G. Bujalesky, M. Fernandez, A. Schiavini, 1995. Evaluación del impacto ambiental del Ferrocarril Austral Fueguino (Tramo: Ex Aserradero Lombardich-Ruta Nacional No 3, Parque Nacional Tierra del Fuego). Presentado ante la Administración de Parques Nacionales.

Dahlen, F. and J. Tromp, 1998. Theoretical global seismology. Princeton university press.

Dalziel, I.W.D., R.H. Dott Jr, R.D. Winn Jr, R.L. Bruhn, 1975. Tectonic relations of South Georgia Island to the southernmost Andes. Geol. Soc. Am. Bull. 86, 1034-1040.

Dalziel, I.W.D., R.L. Brown, 1989. Tectonic denudation of the Darwin metamorphic core complex in the Andes of Tierra del Fuego, southernmost Chile: implications for Codilleran orogenesis. Geology, 17, 699 - 703.

de Wit, M.J., 1977. The evolution of the Scotia Arc as a key of the reconstruction of southern Gondwanaland. Tectonophysics, 37 (1-39), 53-82.

Del Cogliano, D., R. Perdomo, J.L. Hormaechea, E. Olivero, J. Strelin, D. Martinioni, 2000. GPS detection of movements between SCO and SAM plates in the Argentinean part of Tierra del Fuego Island, 31st International Geological Congress, Rio de Janeiro, Brazil.

Derode, A., E. Larose, M. Tanter, J. de Rosny, A. Tourin, M. Campillo, M. Fink, 2003. Recovering the Green's function from field-field correlations in an open scattering medium (L). Journal Acoust. Soc. Am., 113 , 2973-2976.

Dueker, K.G., A.F. Sheehan, 1997. Mantle discontinuity structure from midpoint stacks of converted P to S waves across the Yellowstone hot spot track. J. Geophys. Res., 102, 83138327.

Dziewonski A., S. Bloch, M. Landisman, 1969. A technique for the analysis of transient seismic signals. Bull. Seismol. Soc. Amer., vol. 59, no. 1, pp. 427-444, 1969.

Dziewonski, A.M., Anderson, D.L., 1981. Preliminary reference Earth model. Physics of the Earth and Planetary Interiors, 25, 297-356.

Efron, B., R. Tibshirani, 1991. Statistical Data Analysis in the Computer Age. Science New Series, Vol. 253, No. 5018, pp. 390-395

Ekrem, Z., 2002. The shear wave velocity structure of the eastern Marmara region by using receiver function analysis. PhD thesis, Department of Geophysics, Istanbul Technical 
University.

Febrer, J.M., M.P. Plascencia, N.C. Sabbione, 2000. Local and Regional Seismicity from Ushuaia Broadband Station Observations (Tierra del Fuego). Terra Antartica 8, 35-40.

Febrer, J.M., 2002. La red ASAIN de observación sismológica en Antártida. II. Sismicidad en Tierra del Fuego. XXI Reunión Científica AAGG, Rosario, Argentina.

Ford, H.A., K.M. Fischer, D.L. Abt, C.A. Rychert, L.T. Elkins-Tanton, 2010. The lithosphereasthenosphere boundary and cratonic lithospheric layering beneath Australia from Sp wave imaging, Earth Planet. Sci. Lett., 300, 299-310.

Fouch, M.J., S. Rondenay, 2006. Seismic anisotropy beneath stable continental interiors. Physics of the Earth and Planetary Interiors, 158, 292-320.

Frederiksen, A.W., M.G. Bostock, 2000. Modelling teleseismic waves in dipping anisotropic structures. Geophy. J. Int. 141, 401-412.

Frederiksen, A.W., H. Folsom, G. Zandt, 2003. Neighbourhood inversion of teleseismic Ps conversions for anisotropy and layer dip. Geophysical Journal International, 155(1), 200212.

Friedrich, A., F. Krüger, K. Klinge, 1998. Ocean-generated microseismic noise located with the Gräfenberg array. J. Seismol., 2 , 47-64.

Fuenzalida, R.H., 1972. Geological Correlation Between the Patagonian Andes and the Antarctic Peninsula and Some Tectonic Implications. Master Thesis, Stanford University, 75pp.

Gans, C.R., S.L. Beck, G. Zandt, H. Gilbert, P. Alvarado, M. Anderson, L. Linkimer, L., 2011. Continental and oceanic crustal structure of the Pampean flat slab region, western Argentina, using receiver function analysis: new high-resolution results. Geophys. J. Int., 186, 45-58.

Ghiglione, M.C., 2003. Estructura y evolución tectónica del cretácico terciario de la Costa Atlántica de Tierra del Fuego. Tesis Doctoral, Universidad de Buenos Aires, 196pp.

Gilbert, H.J., A.F. Sheehan, 2004. Images of crustal variations in the intermountain west. Journal of Geophysical Research 109: doi: 10.1029/2003JB002730. Issn: 0148-0227.

Gilbert, H., Beck, S., Zandt, G., 2006. Lithospheric and upper mantle structure of central Chile and Argentina. Geophys. J. Int., 165, 383-398.

Goldstein, P., A. Snoke, 2005. "SAC Availability for the IRIS Community”. Incorporated Institutions for Seismology Data Management Center Electronic Newsletter. http://ds.iris.edu/ds/newsletter/vol7/no1/sacavailability-for-the-iris-community/

Gosselin, J. M., J.F. Cassidy, S.E. Dosso, 2015. Shear-wave velocity structure in the vicinity of the 2012 Mw 7.8 Haida Gwai earthquake from receiver function inversion. Bulletin of the Seismological Society of America, 105, no. 2B, 1106-1113.

Gouédard, P., L. Stehly, F. Brenguier, M. Campillo, Y. Colin de Verdière, E. Larose, L. Margerin, P. Roux, F. J. Sánchez-Sesma, N.M. Shapiro, R.L. Weaver, 2008. Cross-correlation of 
random fields: mathematical approach and applications. Geophysical Prospecting, 56: 375393. doi:10.1111/j.1365-2478.2007.00684.x

Grunow, A., D.V. Kent, I.W.D. Dalziel, 1991. New palaeomagnetic data from Thurston Island: implications for the tectonics of West Antarctica and Weddell Sea opening. J. Geophys.Res. 96, 17935-17954.

Grunow, A.M., I.W.D Dalziel, T.M. Harrison, M.T. Heizer, 1992. Structural geology and geochronology of subduction complexes along the margin of Gondwanaland: New data from Antarctic Peninsula and southnernmost andes. Geological Society of America Bulletin, 104, 1497 - 1514.

Gualtieri, L., E. Stutzmann, Y. Capdeville, F. Ardhuin, M. Schimmel, A. Mangeney, A. Morelli, 2013. Modelling secondary microseismic noise by normal mode summation. Geophys. J. Int., doi:10.1093/gji/ggt090.

Gurrola, H., G.E. Baker, J.B. Minster, 1995. Simultaneous time domain deconvolution with application to the computation of receiver functions. Geophys. J. Int. 120, 537-543.

Hansen, S., K. Dueke, 2009. P- and S-Wave Receiver Function Images of Crustal Imbrication beneath the Cheyenne Belt in Southeast Wyoming Bulletin of the Seismological Society of America, 99:1953-1961.

Hansen, S.E., A.A. Nyblade, J. Julià, P.H.G.M Dirks, J. Raymond, R.J. Durrheim, 2009. Uppermantle low-velocity zone structure beneath the Kaapvaal craton from S-wave receiver functions. Geophys. J. Int. 178, 1021-1027.

Hasselmann, K., 1963. Statistical analysis of the generation of microseisms. Rev. Geophys., 1, 177210.

Haubrich, R. A., W. H. Munk, F. E. Snodgrass, 1963. Comparative spectra of microseisms and swell. Bulletin Geological Society of America, 53 , 27-37.

Heit, B., X. Yuan, M. Bianchi, F. Sodoudi, R. Kind, 2008. Crustal thickness estimation beneath the southern central andes at $30^{\circ} \mathrm{S}$ and $36^{\circ} \mathrm{S}$ from $\mathrm{S}$ wave receiver function analysis. Geophys. J. Int. 174, 249-254.

Herrmann, R. B., 2013. Computer programs in seismology: An evolving tool for instruction and research. Seism. Res. Lettr. 84, 1081-1088.

Hofmann, P., 2015. Solid State Physics. An Introduction, 2nd edition, ISBN-10: 3527412824, ISBN-13: 978-3527412822, Wiley-VCH Berlin.

Jaschek, E., N.C. Sabbione, P. Sierra, 1982. Reubicación de sismos localizados en territorio argentino (1920-1963). Vol. XI de Geofísica, Publicaciones del Observatorio de la Universidad Nacional de La Plata.

Kedar, S., M.S. Longuet-Higgins, F. Webb, N. Graham, R. Clayton, C. Jones, 2008. The origin of deep ocean microseisms in the North Atlantic Ocean, Proceedings of the Royal Society A, 464, 777-793. 
Kikuchi, M, H. Kanamori, 1982. Inversion of complex body waves. Bull. Seismol. Soc. Am. 72, 491-506.

Kind, R., L.P Vinnik, 1988. The upper mantle discontinuities underneath the GRF array from P to S converted phases. J. Geophys. 62, 138-147.

Klepeis, K.A., 1994. The Magallanes and Deseado fault zones: major segments of the South American-Scotia transform plate boundary in southernmost South America, Tierra del Fuego. J. Geophys. Res. 99, 22001-22014.

Kraemer, P. E., 2003. Orogenic shortening and the origin of the Patagonian orocline (56 S. Lat). Journal of South American Earth Sciences, 15(7), 731-748.

Kumar, P., X. Yuan, R. Kind, G. Kosarev, 2005. The lithosphere-asthenosphere boundary in the Tien Shan-Karakoram region from S receiver functions: Evidence for continental subduction. GRL, 32, L07305.

LaCoss, R.T., E.J. Kelly, M.N. Toksöz, 1969. Estimation of seismic noise using arrays. Geophysics, 34 (1), 21-38.

Langston, C.A., 1977. The effect of planar dipping structure on source and receiver responses for constant ray parameter. Bull. Seismol. Soc. Am. 67, 1029-1050.

Langston, C.A., 1979. Structure under Mount Rainier, Washington, inferred from teleseismic body waves. J. Geophys. Res. 84, 4749-4762.

Langston, C.A., 1981. Evidence for the subducting lithosphere under southern Vancouver Island and western Oregon from teleseismic P wave conversions. J. Geophys., 86, 3857-3866.

Larose, E., A. Khan, Y. Nakamura, M. Campillo, 2005. Lunar subsurface investigated from correlation of seismic noise. Geophys. Res. Lett. , 32 (16), L16201.

Laske, G., G. Masters, Z. Ma, M. Pasyanos, 2013. Update on CRUST1.0 - A 1-degree Global Model of Earth's Crust. Geophys. Res. Abstracts, 15, Abstract EGU2013-2658.

Lawrence, J.F. and D.A. Wiens, 2004. Combined receiver-function and surface wave phasevelocity inversion using a niching genetic algorithm; application to Patagonia. Bulletin of the Seismological Society of America, 94(3):977-987.

Lawrence, J. F., P.M. Shearer, 2006. A global study of transition zone thickness using receiver functions. Journal of Geophysical Research-Solid Earth. 111.

Levin, V. and J. Park, 1997. P-SH conversions in a flat-layered medium with anisotropy of arbitrary orientation. Geophysical Journal International, 131(2), 253-266.

Li, X.Q., R. Kind, X.H. Yuan, I. Wolbern, W. Hanka, 2004. Rejuvenation of the lithosphere by the Hawaiian plume. Nature, 427, 827-829.

Ligorria, J. P., C.J. Ammon, 1999. Iterative deconvolution and receiver function estimation. Bull. Seismol. Soc. Am. 89, 1395-1400.

Lin, F.C., M.P. Moschetti, M.H. Ritzwoller, 2008. Surface wave tomography of the western United States from ambient seismic noise: Rayleigh and Love wave phase velocity maps. 
Geophys. J. Int., 173 (1), 281-298.

Lobkis, O.I. and R.L. Weaver, 2001. On the emergence of the Green's function in the correlations of a diffuse field. The Journal of the Acoustical Society of America, 110(6), 3011-3017.

Lodolo, E., M. Menichetti, R. Bartole, Z. Ben-Avraham, A. Tassone, H. Lippai, 2003. MagallanesFagnano continental transform fault (Tierra del Fuego, southernmost South America). Tectonics 22, 1076.

Lodolo, E., M. Menichetti, C. Zanolla, H.F. Lippai, J.L. Hormaechea, A. Tassone, 2007. Gravity map of the Isla Grande de Tierra del Fuego, and morphology of Lago Fagnano. Geologica Acta, 5(4), 0307-314.

Lombardi, D., J. Braunmiller, E. Kissling, D. Giardini, 2008. Moho depth and Poisson’s ratio in the Western-Central Alps from receiver functions. Geophys. J. Int., 173, 249-264.

Lomnitz, C., 1970. Major earthquakes and tsunamis in Chile. Geologische Rundschau 59, 938-960.

Longuet-Higgins, M.S., 1950. A theory of the origin of microseisms. Phil. Trans. R. Soc. Lond. A, 243, 1-35.

Ludwig, W. J., J.I. Ewing, M. Ewing, 1965. Seismic Refraction measurements in the Magellan Straits. J. Geophys. Res, 70, 1855-76.

Mangino, S.G., G. Zandt, C.J. Ammon, 1993. The Receiver Structure Beneath Mina, Nevada. Bull. Seismol. Soc. Am. 83, 542-560.

McNamara, D.E. and T.J. Owens, 1993. Azimuthal shear wave velocity anisotropy in the Basin and Range Province using moho Ps converted phases. J. Geophys. Res., 98, 12003-12007.

McNamara, D.E. and R.P. Buland, 2004. Ambient noise levels in the continental United States. Bulletin of the seismological society of America, 94(4), 1517-1527.

Megna, A. and A. Morelli, 1994. Determination of Moho depth and dip beneath MEDNET station AQU by analysis of broadband receiver stations. Annali di Geofisica, Vol. XXXVII, No5.

Mendoza, L., R. Perdomo, J.L. Hormaechea, D. Del Cogliano, M. Fritsche, A. Richter, R. Dietrich, 2011. Present-day crustal deformation along the Magallanes-Fagnano Fault System in Tierra del Fuego from repeated GPS observations. Geophys. J. Int. 184, 1009-1022.

Mendoza, L., A. Richter, M. Fritsche, J. L. Hormaechea, R. Perdomo and R. Dietrich, 2015. Block modeling of crustal deformation in Tierra del Fuego from GNSS velocities. Tectonophysics 651-652 (2015) 58-65.

Menichetti, M., E. Lodolo, A. Tassone, 2008. Structural geology of the Fuegian Andes and Magallanes fold-and -thrust belt-Tierra del Fuego Island. Geologica Acta 6, 19-42.

Mottaghi A.A, M. Rezapour, M Korn, 2013. Ambient noise surface wave tomography of the Iranian Plateau. Geophysical Journal International 193 (1), 452-462.

Mulargia, F., 2012. The seismic noise wavefield is not diffuse. Journal Acoust. Soc. Am., 131, (4), 2853-2858.

Nishida, K., J.P. Montagner, H. Kawakatsu, 2009. Global surface wave tomography using seismic 
hum. Science, 326 (5949), 112, doi: 10.1126/science.1176389.

Oldenburg, D.W, 1981. A comprehensive solution to the linear deconvolution problem. J. Geophys. J. R. Astr. Soc. 65, 331-357.

Olivero, E.B., D.R. Martinioni, 2001. A review of the geology of the Argentinian Fuegian Andes. J. South Amer. Earth Sci. 14, 175-188.

Oreshin, S., L. Vinnik, D. Peregoudov, S. Roecker, 2002. Lithosphere and asthenosphere of the Tien Shan imaged by S receiver functions. Geophysical research letters, 29(8).

Owens, T.J., G. Zandt, S.R. Taylor, 1984. Seismic evidence for an ancient rift beneath the Cumberland Plateau, Tennessee: a detailed analysis of broadband teleseismic P waveforms. J. Geophys. Res., 89, 7783-7795.

Owens, T. J., S. R. Taylor, G. Zandt, 1987. Crustal structure at regional seismic test network stations determined from inversion of broadband teleseismic P waveforms, Bull. Seism. Soc. Am., 77, 631-632.

Owens, T.J., R.S. Crosson, M. Hendrickson, 1988. Constraints on the subduction geometry beneath western Washington from broadband teleseismic waveform modeling. Bull. Seis. Soc. Am., 78, 1319-1334.

Paul A., M. Campillo, L. Margerin, E. Larose and A. Derode, 2005. Empirical synthesis of timeasymmetrical Green functions from the correlation of coda waves. J. Geophys. Res., 110, B08302.

Paulssen, H., E. Stutzmann, 1996. On PP-P differential travel time measurements. Geophys. Res. Lett., vol 23 n 14, 1833-1836.

Peng, X., E.D. Humphreys, 1997. Moho dip and crustal anisotropy in Northwestern Nevada from teleseismic receiver functions. Bull. Seismol. Soc. Am. 87, 745-754.

Perarnau, M., P. Alvarado, M. Saez, 2010. Estimación de la estructura cortical de velocidades sísmicas en el suroeste de la Sierra de Pie de Palo, Provincia de San Juan. Rev. Asoc. Geol. Argent., 64, 473-480.

Peroni, J.I., A.A. Tassone, M. Menichetti, M.E. Cerredo, 2009. Geophysical modeling and structure of Ushuaia pluton, Fuegian Andes, Argentina. Tectonophysics, 476(3), 436-449.

Peterson, J., 1993. Observation and modeling of seismic background noise. Open-file report 93-322 Rep., U.S. Geological Survey, Alburquerque, New Mexico, 42 pp.

Phinney, R.A., 1964. Structure of earths crust from spectral behavior of long-period body waves. J. Geophys. Res. 69, 2997-3017.

Piana Agostinetti, N., F.P. Lucente, G. Selvaggi, M. Di Bona, 2002. Crustal structure and Moho geometry beneath the Northern Apennines (Italy). Geophysical Research Letters, 29(20).

Piana Agostinetti, N. and A. Amato, 2009. Moho depth and Vp/Vs ratio in peninsular Italy from teleseismic receiver functions. Journal of Geophysical Research, 114, B06303, doi:10.1029/2008JB005899. 
Plascencia, M., G. Connon, J.L. Hormaechea, N.C. Sabbione, 2002. Determinación preliminar de epicentros registrados en la estación sismológica DSPA (Despedida), Provincia de Tierra del Fuego. XXI Reunión Científica AAGG, 285-289.

Rickett, J. and J.F. Claerbout, 1999. Acoustic daylight imaging via spectral factorization: helioseismology and reservoir monitoring. The Leading Edge , 18 , 957-960.

Robertson Maurice, S. D., D. A. Wiens, K. D. Koper, E. Vera, 2003. Crustal and upper mantle structure of southernmost South America inferred from regional waveform inversion. J. Geophys. Res., 108(B1), 2038.

Roux, P., K.G. Sabra, P. Gerstoft, W. A. Kuperman, M.C. Fehler, 2005. P-waves from crosscorrelation of seismic noise. Geophys. Res. Lett., 32 (19), L19303.

Ryan, J., S. Beck, G. Zandt, L. Wagner, E. Minaya, H. Tavera, 2016. Central Andean crustal structure from receiver function analysis. Tectonophysics, 682, 120-133.

Rychert, C.A., S. Rondenay, K.M. Fischer, 2007. P-to-S and S-to-P imaging of a sharp lithosphereasthenosphere boundary beneath eastern North America. J. Geophys. Res., 112, B08314.

Rychert, C.A. and N. Harmon, 2016. Stacked P-to-S and S-to-P receiver functions determination of crustal thickness, Vp and Vs: The H-V stacking method. Geophys. Res. Lett., 43, 14871494.

Sabbione, N.C., G. Connon, J.L. Hormaechea, C. Buffoni, 2007a. Tierra del Fuego Reference Standard Earthquake Catalogue. International Geological Congress on the Southern Hemisphere Santiago del Chile. Geosur 2007.

Sabbione, N.C., G. Connon, J.L. Hormaechea, M.L. Rosa, 2007b. Estudio de sismicidad en la provincia de Tierra del Fuego, Argentina. Geoacta 32, 41-50.

Sabra, K.G., P. Gerstoft, P. Roux, W. A. Kuperman, M. C. Fehler, 2005a. Extracting time-domain Green's function estimates from ambient seismic noise. Geophys. Res. Lett., 32 (3), L03310, doi:10.1029/2004GL021862.

Sabra, K.G., P. Gerstoft, P. Roux, W. A. Kuperman, M.C. Fehler, 2005b. Surface wave tomography from microseisms in Southern California. Geophys. Res. Lett., 32 (14), L14311.

Sambridge, M. and B. Kennett, 1996. Genetic algorithm inversion for receiver functions with application to crust and uppermost mantle structure. Geophysical Research Letters, 23(14), 1829-1832.

Sambridge, M., 1999. Geophysical Inversion with a Neighbourhood Algorithm I. Searching a parameter space. Geophys. J. Int., 138, 479-494, 1999.

Savage, M.K., 1998. Lower Crustal anisotropy or dipping boundary? Effects on receiver functions and a case study in New Zealand. J. Geophys. Res. 103, 15069-15089.

Schimmel, M. and H. Paulssen, 1997. Noise reduction and detection of weak, coherent signals through phase weighted stacks. Geophys. J. Int. , 130 , 497-505.

Schimmel M., 1999. Phase cross-correlations: design, comparisons and applications. Bull. Seismol. 
Soc. Am., 89, 1366-1378.

Schimmel M. and J. Gallart, 2005. The inverse S Transform in filters with time-frequency localization. IEEE Transactions on Signal Processing, 53 (11), 4417 - 4422, doi:10.1109/TSP.2005.857065.

Schimmel, M., E. Stutzmann, J. Gallart, 2011. Using instantaneous phase coherence for signal extraction from ambient noise data at a local to a global scale. Geophys. J. Int., 184, 494506.

Shapiro, N.M. and M. Campillo, 2004. Emergence of broadband Rayleigh waves from correlations of the ambient seismic noise. Geophysical Research Letters, 31(7).

Shapiro, N.M., M. Campillo, L. Stehly, M.H. Ritzwoller, 2005. High-resolution surface-wave tomography from ambient seismic noise. Science , 307 (5715), 1615-1618.

Shibutani, T., M. Sambridge, B. Kennett, 1996. Genetic algorithm inversion for receiver functions with application to crust and uppermost mantle structure beneath East Australia. Geophys. Res. Lett. 23, 1829-1832.

Smalley, R., E. Kendrick, M.G. Bevis, I.W.D Dalziel, F. Taylor, E. Laura, R. Barriga, G. Casassa, E. Olivero and E. Piana, 2003. Geodetic determination of relative plate motion and crustal deformation across the Scotia-South America plate boundary in eastern Tierra del Fuego. Geochem. Geophys. Geosyst. 4, doi:10.1029/2002GC000446.

Simon C., S. Ventosa, M. Schimmel, A. Heldring, J.J. Danobeitia, J. Gallart, A. Manuel, 2007. The S-Transform and its inverses: side effects of discretizing and filtering. IEEE Trans. Signal Process., Vol. 55 , pp. 4928-4937, doi 10.1109/TSP.2007.897893.

Snieder, R., 2004. Extracting the Green's function from the correlation of coda waves: a derivation based on stationary phase. Phys. Rev. E., 69 (046610), 1-8.

Snieder, R., K. Wapenaar, U. Wegler, 2007. Unified Green's function retrieval by cross-correlation; connection with energy principles. Phys. Rev. E, 75, 036103.

Stanciu, A.C., R.M. Russo, V.I. Mocanu, P.M. Bremner, S. Hongsresawat, M. E. Torpey, J.C. Vandecar, D.A. Foster, J.A. Hole, 2016. Crustal structure beneath the Blue Mountains terranes and cratonic North America, eastern Oregon, and Idaho, from teleseismic receiver functions. J. Geophys. Res. Solid Earth, 121, 5049-5067.

Stehly, L., M. Campillo, N.M. Shapiro, 2006. A study of the seismic noise from its long-range correlation properties. J. Geophys. Res., 111 (B10), B10306, doi: 10.1029/2005JB004237.

Stockwell, R.G., L. Mansinha, R.P. Lowe, 1996. Localization of the complex spectrum: the S transform. IEEE transactions on signal processing, 44(4), 998-1001.

Stutzmann, E., M. Schimmel, G. Patau, A Maggi, 2009. Global climate imprint on seismic noise. Geochemistry, Geophysics, Geosystems, 10(11).

Stutzmann, E., F. Ardhuin, M. Schimmel, A. Mangeney, G. Patau, 2012. Modelling long-term 
seismic noise in various environments. Geophys. J. Int., doi: 10.1111/j.1365246X.2012.05638.x.

Tassone, A., H. Lippai, E. Lodolo, M. Menichetti, A. Comba, J.L. Hormaechea, J.F. Vilas, 2005. A geological and geophysical crustal section across the Magallanes-Fagnano fault in Tierra del Fuego and associated asymmetric basins formation. J. South Am. Earth Sci. 19, 99-109.

Tanimoto, T., 2007. Excitation of microseisms. Geophys. Res. Lett., 34 (5), L05308.

Thompson, D.A., I.D. Bastow, G. Helffrich, J.M. Kendall, J. Wookey, D.B. Snyder, D.W. Eaton, 2010. Precambrian crustal evolution: Seismic constraints from the Canadian Shield. Earth Planetary Science Letters 297, 655-666.

Tomfohrde, D., R. Nowack, 2000. Crustal Structure Beneath Taiwan Using Frequency-by Inversion of Receiver Function Waveforms. Pure appl. Geophys, 157, 737.

Tomlinson, J.P., P. Denton, P.K.H. Maguire, D.C. Booth, 2006. Analysis of the crustal velocity structure of the British Isles using teleseismic receiver functions. Geophys. J. Int., 167, 223-237.

Torres-Carbonell, P.J., E.B. Olivero, L.V. Dimieri, 2008. Control en la magnitud de desplazamiento de rumbo del Sistema Transformante Fagnano, Tierra del Fuego, Argentina. Revista Geológica de Chile 35 (1): 63-77.

Torres-Carbonell, P.J. and L.V. Dimieri, 2013. Cenozoic contractional tectonics in the Fuegian Andes, southernmost South America: a model for the transference of orogenic shortening to the foreland. Geologica Acta, Vol. 11, №3.

Velasco, M.S, M. Ellis, R. Jr. Smalley, 2002. Active faulting in southern Tierra del Fuego. Seismol. Res. Lett. 73:419.

Vinnik L.P., 1977. Detection of waves converted from P to SV in the mantle, Phys. Earth planet. Inter., 15, 294-303.

Vinnik, L.P., V. Farra, R. Kind, 2004a. Deep structure of the Afro-Arabian hotspot by S receiver functions. Geophysical Research Letters, 31(11).

Vinnik, L.P., C. Reigber, I.M. Aleshin, G.L. Kosarev, M.K. Kaban, D.I. Oreshin, S. Roecker, 2004b. Receiver function tomography of the central Tien Shan. Earth and Planetary Science Letters, 225(1), 131-146.

Vinnik, L.P., I.M. Aleshin, M.K. Kaban, S.G. Kiselev, S.G., G.L. Kosarev, S.I. Oreshin, C. Reigber, 2006. Crust and mantle of the Tien Shan from data of the receiver function tomography. Izvestiya Physics of the Solid Earth, 42(8), 639-651.

Wang, P., L. Wang, N. Mi, J. Liu, H.Li, D. Yu, M. Xu, X. Wang, Z. Guo, 2010. Crustal thickness and average $\mathrm{Vp} / \mathrm{Vs}$ ratio variations in southwest Yunnan, China, from teleseismic receiver functions. Journal of Geophysical Research, 115, B11308.

Wapenaar, K., 2004. Retrieving the elastodynamic Green's function of an arbitrary inhomogeneous medium by cross correlation. Physical Review Letters , 93 , 254391-254395. 
Wapenaar, K., E. Slob, R. Snieder, 2006. Unified Green's function retrieval by cross correlation. Physical Review Letters, 97 (234301), 1-4.

Weaver, R.L., 2005. Information from seismic noise. Science, 307 , 1568-1569.

Webb, S.C., 2007. The Earth's 'hum' is driven by ocean waves over the continental shelves. Nature, 445 (7129), 754-756.

Wessel, P., W.H.F Smith, 1995. New version of the generic mapping tools released, American Geophysical Union, http://www.agu.org/eos elec.

Wiggins, R.A., 1969. Monte Carlo inversion of body wave observations. J. geophys. Res., 74, 3171-3181.

Young, Ch. J., E.P. Chael, M.W. Withers, R.C. Aster, 1996. A comparison of the high-frequency $(>1 \mathrm{~Hz})$ surface and subsurface noise environment at three sites in the United States. Bull. Seism. Soc. Am., 86, 5, 1516-1528.

Yuan, X., J. Ni, R. Kind, E. Sandvol, J. Mechie, 1997. Lithospheric and upper mantle structure of southern Tibet from a seismological passive source experiment. J Geophys Res., 102, 27491-27500.

Zandt, G. and C.J. Ammon, 1995. Continental crust composition constrained by measurements of crustal Poisson's ratio. Nature 374, 152-154.

Zhang, J., C.A. Langston, 1995. Dipping structure under Dourbes, Belgium, determined by receiver function modeling and inversion. Bull. Seismol. Soc. Am. 85, 254-268.

Zhao, L.S., M.K. Sen, P. Stoffa, C. Frohlich, 1996. Application of very fast simulated annealing to the determination of the crustal structure beneath Tibet. Geophysical Journal International, 125(2), 355-370.

Zhu, L., 1993. Estimation of crustal thickness and $\mathrm{V}_{\mathrm{p}} / \mathrm{V}_{\mathrm{s}}$ ratio beneath the Tibetan Plateau from teleseismic converted waves (abstract). Eos Trans. AGU, 7416, Spring Meet. Suppl., 202.

Zhu, L., H. Kanamori, 2000. Moho depth variation in southern California from teleseismic receiver functions. J. Geophys. Res. 105, 2969-2980.

Zhu, L., 2004. Lateral variation of the Tibetan lithospheric structure inferred from teleseismic waveforms, in Advancements in Seismology and Physics of the Earth Interior in China, Seismology Press, Beijing.

Zor, E., E. Sandvol, C. Gürbüz, N. Türkelli, D. Seber, M. Barazangi, 2003. The crustal structure of the East Anatolian plateau (Turkey) from receiver functions. Geophysical Research Letters, 30(24). 